\title{
Neoproterozoische bis paläozoische Krustendynamik am Westrand des Río de la Plata Kratons
}

\author{
Dissertation \\ zur Erlangung des Doktorgrades \\ der Mathematisch-Naturwissenschaftlichen Fakultäten \\ der Georg-August-Universität zu Göttingen
}

vorgelegt von

Malte Drobe

aus Hannover

Göttingen 2009 



\title{
Zusammenfassung
}

Um Hinweise auf die Provenienz und die geodynamischen Entwicklung der östlichen Sierras Pampeanas und der Cordillera Oriental zu erhalten wurden geochemische Untersuchungen der Haupt- und Spurenelemente in Kombination mit der Sm-Nd und Pb-Pb Isotopie sowie SHRIMP Altern an detritischen Zirkonen der Sierra de San Luis, den Sierras de Córdoba, den Sierras de Chepes, der Sierra Norte und der Puncoviscana Formation durchgeführt. Diese unterschiedlichen Methoden wurden kombiniert um ein besseres Verständnis des Arbeitsgebietes zu erlangen. Die geochemischen - und die Sm-Nd Daten deuten auf einen etwas stärkeren vulkanischen Einfluss in den Sierras de Córdoba im Vergleich zu den anderen Einheiten hin. Die $\mathrm{T}_{\mathrm{DM}}$ Modellalter der Sierras de Chepes ( 1,82 Ga) und der Sierra Norte ( 1,79 Ga) sind signifikant älter als die Daten aus den Sierras de Córdoba ( 1,67 Ga). Der Grund hierfür könnte in der hochgradigen Metamorphose liegen, die auch die ${ }^{208} \mathrm{~Pb} /{ }^{204} \mathrm{~Pb}$ Verhältnisse der Sierras de Córdoba zumindest teilweise beeinflusst zu haben scheint. Ein spät pampeaner Peak in den detritischen Zirkonaltern der Sierras de Chepes um 520 Ma stimmt mit den neuen Altern der San Luis Formation überein und wurde in der Literatur ebenso in dem nordwestlich der Sierras de Chepes liegenden Famatina Belt und in der nördlich gelegenen Mesón Group nachgewiesen. Diese Alter liegen auch in einigen Bereichen der Puncoviscana Formation vor, fehlen jedoch gänzlich in den Sierras de Córdoba. In dieser Arbeit werden ein verbessertes Modell der geodynamischen Entwicklung der östlichen Sierras Pampeanas und der Cordillera Oriental sowie ein neues Modell der spät pampeanen Entwicklung der Sierra de San Luis vorgestellt um neue Einblicke in die komplexe Entwicklung der östlichen Sierras Pampeanas und der Cordillera Oriental zu erhalten.

\begin{abstract}
Whole rock geochemical analyses using major and trace elements in combination with the $\mathrm{Sm}-\mathrm{Nd}$ and $\mathrm{Pb}-\mathrm{Pb}$ isotope systems together with SHRIMP age dating on metasedimentary rocks from the Sierras de Chepes, the Sierras de Córdoba and the Sierra Norte and the San Luis Formation in the Sierra de San Luis have been carried out to unravel the Provenance and the geodynamic history of the Eastern Sierras Pampeanas. The geochemical and the Sm-Nd data point to a slightly stronger volcanic influence in the Sierras de Córdoba compared to the other units. The TDM ages from the Sierras de Chepes $(\sim 1.82 \mathrm{Ga})$ and the Sierra Norte $(\sim$ $1.79 \mathrm{Ga}$ ) are significantly older than the data from the Sierras de Córdoba (1.67 Ga). High grade metamorphism also seems to have effected the $208 \mathrm{~Pb} / 204 \mathrm{~Pb}$ ratios of at least some samples from the Sierras de Córdoba. A late Pampean detrital zircon peak around 520 Ma
\end{abstract}


from the Sierras de Chepes is in accordance with the new data from the San Luis Formation, literature data from the Famatina Belt located to the northwest of the Sierras de Chepes and also fit detrital zircon peaks in the Mesón group. These ages were also reported from some locations in the Puncoviscana Formation, but are absent in the Sierras de Córdoba. An improved model for the development of the Eastern Sierras Pampeanas in the area between the Sierras de Córdoba and the Puncoviscana Formation and a new insight into the late Pampean development of the Sierra de San Luis will be provided to give new insights in the complex development of the Eastern Sierras Pampeanas. 


\section{Inhaltsverzeichnis}

1 Einleitung und Zielsetzung $\quad 1$

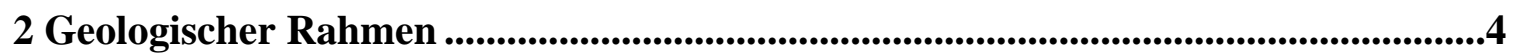

3 Beschreibung des Probenmaterials..........................................................................17

3.1 Nogolí Metamorphic Complex ..................................................................18

3.2 Pringles Metamorphic Complex …...............................................................18

3.3 Conlara Metamorphic Complex...................................................................19

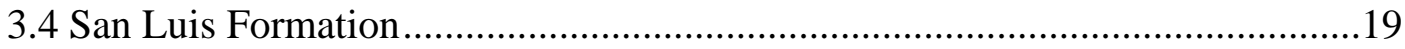

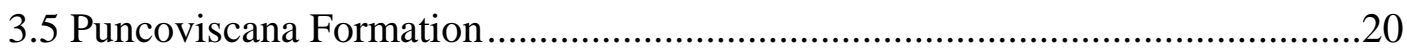

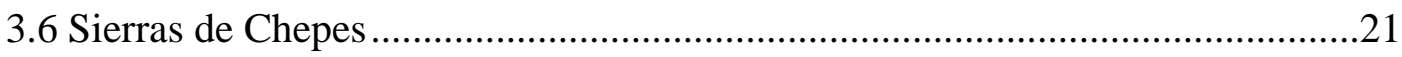

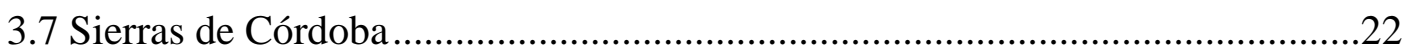

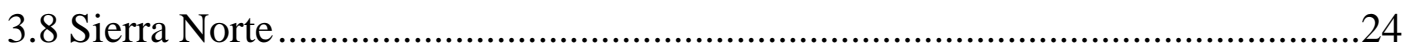

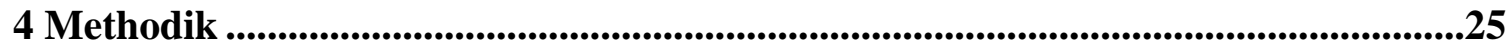

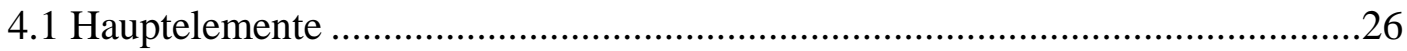

4.2 Aufschlüsse der Spurenelemente .............................................................26

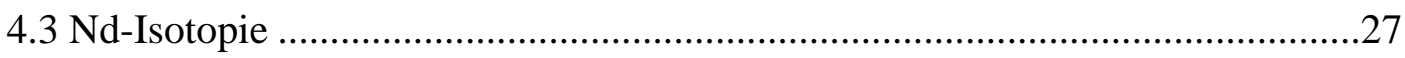

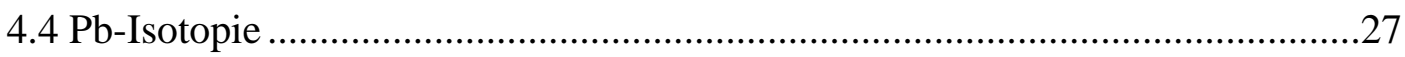

4.5 U-Pb SHRIMP Zirkondatierungen ............................................................28

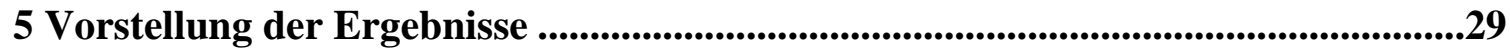

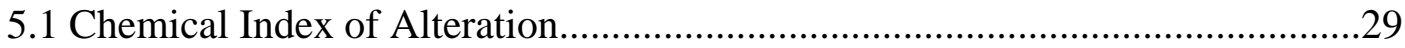

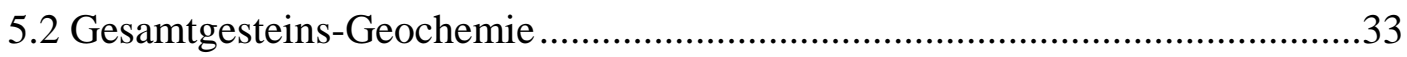

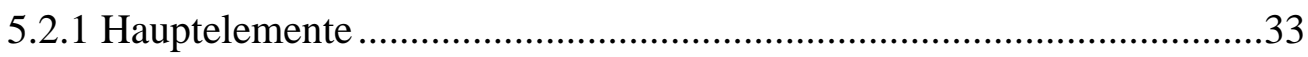

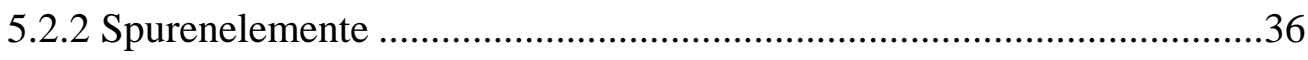

5.2.3 Seltene Erdelemente REE.................................................................40

5.2.4 Geotektonische Position ....................................................................45

5.3 Ergebnisse der Sm-Nd WR Isotopenanalysen .............................................50

5.4 Ergebnisse der Pb-Pb WR Isotopenanalysen.................................................53

5.5 Zirkon Geochronologie: U-Pb SHRIMP Datierungen......................................56 


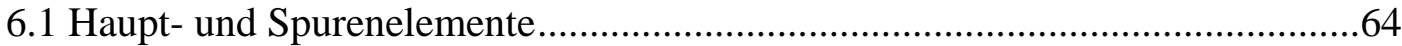

6.2 Schlussfolgerungen aus den Sm-Nd WR Daten .............................................66

6.3 Schlussfolgerungen aus den Pb-Pb WR Daten .............................................71

6.4 Schlussfolgerungen aus den U-Pb SHRIMP Altersdatierungen .........................72

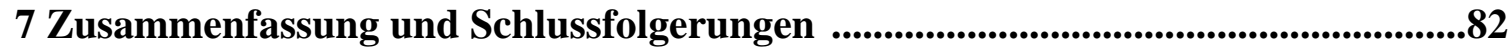

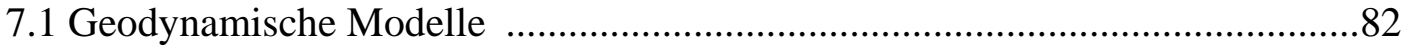

7.1.1 Mögliche Liefergebiete der (Meta)Sedimente der östlichen Sierras Pampeanas

7.1.2 Geodynamische Entwicklung der Sierras de Córdoba und der Puncoviscana Formation 84

7.1.3 Spät-pampeane Entwicklung der östlichen Sierras

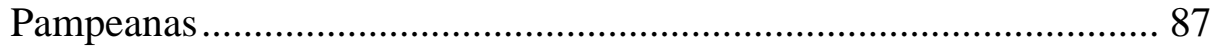

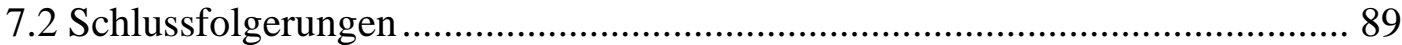

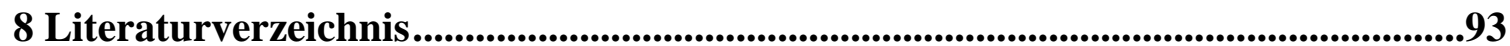

\section{Anhang}




\section{Vorwort}

Herrn Prof. Dr. Siegesmund möchte ich ganz herzlich für die Übernahme des Referates dieser Arbeit danken. Außerdem möchte ich mich für seine fortwährende Motivation während meiner Zeit als Doktorand bedanken.

Ganz herzlich möchte ich mich bei Herrn Prof. Dr. Robert Frei für die Übernahme des Korreferates Bedanken, das er trotz knapp bemessener Zeit übernommen hat. Zusätzlich möchte ich mich für die Hilfe bei Aufschlüssen und Messungen der Pb-Isotopie bedanken.

Bei Herrn Dr. Klaus Wemmer möchte ich mich für die vielfältige Unterstützung während meiner Zeit in Göttingen bedanken, besonders für seinen kritischen Blick auf vermeintliche Details, die aber dennoch oft essentiell bei der Auswertung von Daten waren.

Besonderer Dank gilt Herrn Dr. André Steenken, der mir in der Probenahmezeit in Argentinien sehr geholfen hat und auch in der Zeit danach zu wichtigen Diskussionen über die Geodynamik Argentiniens zur Seite stand.

In gleichem Atemzug ist Frau Dra. Mónica López de Luchi zu nennen, die durch fortwährende Anregungen in Bezug auf die Datenauswertung und ihre Interpretation stets ein wichtiger Ansprechpartner war.

Bei Herrn Dr. Pedro Oyhantcabal und Herrn Dr. Roberto Martino möchte ich mich für die Hilfe bei der Probenahme bedanken.

Bei Herrn Dr. Ulrich Hein möchte ich mich für das Korrekturlesen zum Abschluss bedanken, obwohl ich sehr kurzfristig an ihn herangetreten bin.

Vielen Dank auch an meine Kollegen, Kommilitonen und Freunde in Göttingen, die mich stets unterstützt haben. Besonders möchte ich hierbei Nicole für das Lesen in der letzten Woche danken.

Der Deutschen Forschungsgemeinschaft danke ich für die finanzielle Unterstützung im Rahmen des Projektes Si 438/28-1;2. Gleiches gilt für den Deutschen akademischen Austauschdienst, der mich mit einem Kurzstipendium für Doktoranden unterstützte.

Ein Großteil der hier vorgestellten Daten wurde bereits in zwei Publikationen (Drobe et al. 2009, Drobe et al. in review) publiziert. Wenn Daten dieser Publikationen in dieser Arbeit verwendet werden, findet kein weiterer Verweis darauf statt. 


\section{Einleitung und Zielsetzung}

Die geodynamische Entwicklung des Südwestrandes von Gondwana wird auf der Grundlage geochemischer und isotopengeologischer Untersuchungen seit mehr als einer Dekade kontrovers diskutiert. Im Fokus stehen hier Provenienzanalysen von (Meta)Sedimenten (Rapela et al. 1998; Sims et al. 1998; López de Luchi et al. 1999, 2003; Bock et al. 2000; Lucassen et al. 2000; Brogioni 2001; Aceñolaza et al. 2002; Cerredo und López de Luchi 2002; Thomas et al. 2002; Thomas und Astini 2003; Finney et al. 2003; Schwartz und Gromet 2004; Steenken et al. 2004, 2006; Zimmermann 2005; Rapela et al. 2007; Escayola et al. 2007; Adams et al. 2008) in den östlichen Sierras Pampeanas und der Cordillera Oriental (Abb. 1).

Unterschiedliche geotektonische Milieus wie passiver oder aktiver Kontinentalrand, aber auch die Frage, ob die Einheiten autochthon oder allochthon sind, wurden bei den neoproterozoischen bis früh paläozoischen metasedimentären Einheiten der östlichen Sierras Pampeanas und der Cordillera Oriental, den beiden wichtigsten morphotektonischen Einheiten des Südwestrandes Gondwanas in Argentinien diskutiert (z.B. Ramos 1988; Bahlburg 1990; Astini et al. 1995; Pankhurst und Rapela 1998; Rapela et al. 1998, 2007; Bock et al. 2000; Lucassen et al. 2000; Zimmermann und Bahlburg 2003; López de Luchi et al. 2003; Schwartz und Gromet 2004; Steenken et al. 2004, 2006; Prozzi und Zimmermann 2005; Zimmermann 2005; Schwartz et al. 2008; Adams et al. 2008; Casquet et al. 2008; Siegesmund et al. 2009). Geochemische und isotopengeochemische Untersuchungen unterstützen die Vermutung, dass die niedrig- bis hochgradigen metamorphen Einheiten in der Sierra de San Luis, den Sierras de Chepes, den Sierras de Córdoba und der Sierra Norte die südliche Verlängerung der sehr niedrig- bis niedriggradigen Einheiten der Cordillera Oriental im Nordwesten Argentiniens und im südlichen Bolivien sind (z.B. Schwartz und Gromet 2004; Steenken et al. 2004; Zimmermann 2005; Siegesmund et al. 2009). Aufgrund der pampeanen Orogense im Neoproterozoikum bis Kambrium wurden die östlichen Sierras Pampeanas metamorph überprägt, deformiert und von magmatischen Körpern intrudiert (Toselli und Aceñolaza 1978; Aceñolaza und Toselli 1981; Omarini 1983; Aceñolaza et al. 1988, 1990; Rapela et al. 1998, 2007; Bock et al. 2000; Steenken et al. 2006, 2007; Zimmermann 2005; Siegesmund et al. 2009).

Geochemische Untersuchungen an (Meta)Sedimenten werden vorgenommen, um Informationen über das geotektonische Milieu zu erlangen und gleichzeitig Hinweise auf die 
Liefergebiete der abgelagerten Sedimente zu bekommen. Solche Untersuchungen können mit folgenden Methoden durchgeführt werden:

(i) statistischen Untersuchungen von Lithoklasten mit Hilfe von Dünnschliffen (Dickinson und Suczek 1979; Zimmermann 1999; von Eynatten et al. 2003);

(ii) der Betrachtung der geochemischen Zusammensetzung und der Elementverteilung in Mineralen und Gesamtgesteinsproben (Floyd und Leveridge 1987; McLennan et al. 1990, 1993; Zimmermann 1999);

(iii) Studien über die Isotopie von Gesamtgesteinsproben im Hinblick auf die Verhältnisse der Pb-Isotopie, der Sm-Nd-Isotopie und auch Altersdatierungen wie z.B. die U-Pb Einzelkorndatierung von Zirkonen (Rapela et al. 1998; Sims et al. 1998; Bock et al. 2000; Lucassen et al. 2001, 2002; Steenken et al. 2004, 2006). Diese Methoden können bei der Lösung vieler Probleme bezüglich der Liefergebiete klastischer Sedimente aber auch der Einordnung von geodynamischen Prozessen und der geodynamischen Entwicklung behilflich sein.

Der Vorteil einiger Spurenelemente wie der high field strength elements (HFSE) und der seltenen Erdelemente (REE) besteht darin, unter Oberflächenbedingungen, Erosion, Diagenese und Metamorphose nur schwach löslich zu sein oder sich zumindest ähnlich zu verhalten wie die übrigen Elemente der gleichen Elementgruppen. Bei Hauptelementen ist dies nicht unbedingt gegeben, da sie wesentlich anfälliger auf Lösungsprozesse bei der Erosion und des Transports sowie der Metasomatose während der Diagenese und der Metamorphose reagieren. Aus diesen Gründen sind Spurenelemente bei Provenienzanalysen und der Charakterisierung des geotektonischen Milieus verlässlicher (z.B. Bhatia 1983; Taylor und McLennan 1985; McLennan et al. 1993; Roser et al. 1996). In den meisten Fällen werden Elemente wie die REEs, Ni, Y, Sc, Cr, Th, V, Ti, Hf und Zr verwendet, um Aussagen über das geotektonische Milieu bzw. das Liefergebiet zu treffen. Zusätzlich können Hinweise über den Anteil an basischem und saurem Material oder die Stärke von Recyclingprozessen aufgrund charakteristischer Elementverhältnisse gegeben werden. Dies ist zwar auch mit Hauptelementen möglich, allerdings sollten diese Ergebnisse kritisch betrachtet werden, da sie aufgrund der höheren Mobilität anfälliger für verfälschte Verhältnisse sind, die dann die geodynamische Position bzw. die Geochemie des Liefergebietes nicht richtig widerspiegeln. Auch wenn lediglich Spurenelemente verwendet werden, ist eine Aussage bezüglich der Provenienz und/oder der tektonischen Position allein basierend auf diesen geochemischen Ergebnissen schwierig. Diese Ergebnisse sollten immer mit weiteren Analysen wie z.B. der Pb-Isotopie oder Sm-Nd Daten verglichen werden, um verlässlichere Daten für eventuell 
unterschiedliche Liefergebiete zu erhalten. Ein zusätzliches Hilfsmittel zur Lösung dieser Fragen kann die U-Pb SHRIMP Datierung von Zirkonen darstellen. Aufgrund der Stabilität dieser Minerale ist es möglich, in einer Probe oder sogar einem Korn Hinweise auf mehrere orogene Ereignisse zu erhalten. Gewisse Liefergebiete können also aufgrund ihrer Altersstruktur direkt ausgeschlossen werden, auch wenn die geochemischen Daten wie auch die Pb-Isotopie und Nd-Isotopie ähnlich sind.

Ziel der vorliegenden Arbeit ist es, einen Einblick in die neoproterozoische bis kambrische geodynamische Entwicklung des Westrandes von Gondwana in Argentinien zu gewinnen. Deshalb wurden geochemische Analysen von Haupt- und Spurenelementen, die Isotopverhältnisse von $\mathrm{Pb}$ und Sm-Nd sowie SHRIMP U-Pb Alter an Zirkonen kombiniert, um einen Beitrag zur Entschlüsselung der Provenienz und des geotektonischen Milieus verschiedener Einheiten der Puncoviscana Formation (Cordillera Oriental) und teilweise höhergradiger, metasedimentärer Gesteine der Sierras de Córdoba, Sierra Norte, Sierras de Chepes und der Sierra de San Luis zu geben (Abb. 1). Bereits vorher aufgestellte Modelle zur geodynamischen Entwicklung dieses Gebietes werden durch die Kombination der neuen Daten mit den aus der Literatur zur Verfügung stehenden kritisch diskutiert (Rapela et al.1998, 2007; Sims et al. 1998; López de Luchi et al. 1999, 2003; Bock et al. 2000; Brogioni 2001; Steenken et al. 2004, 2006; Zimmermann 2005; Escayola et al. 2007; Adams et al. 2008; Siegesmund et al. 2009). 


\section{Geologischer Rahmen}

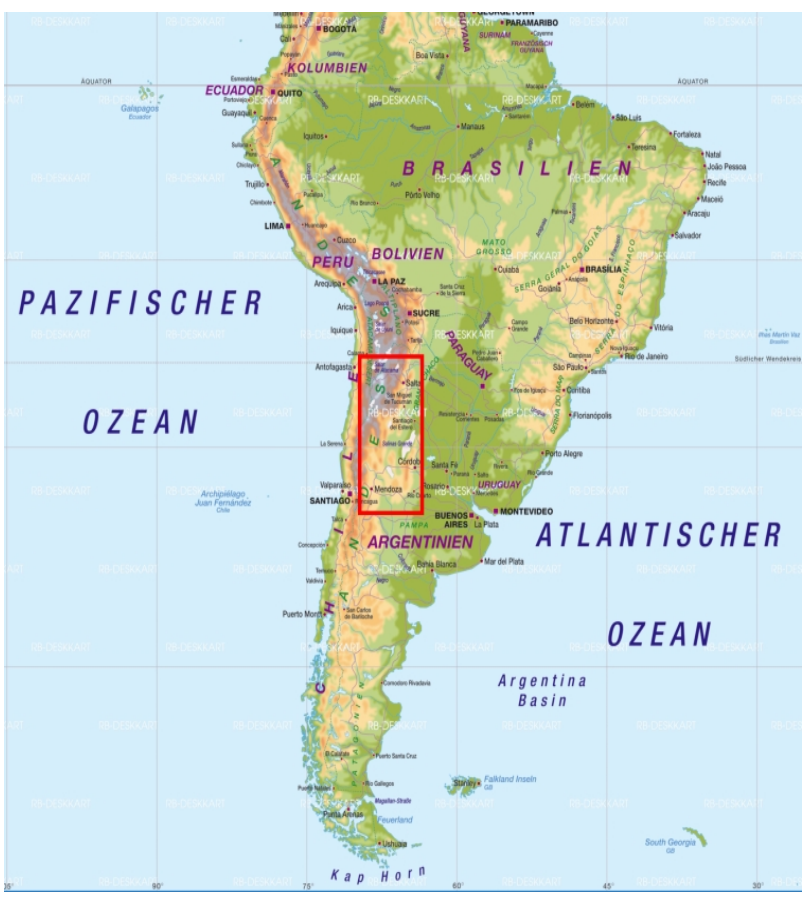

Abbildung 1: Lage des Arbeitsgebietes (rotes Rechteck) in Südamerika

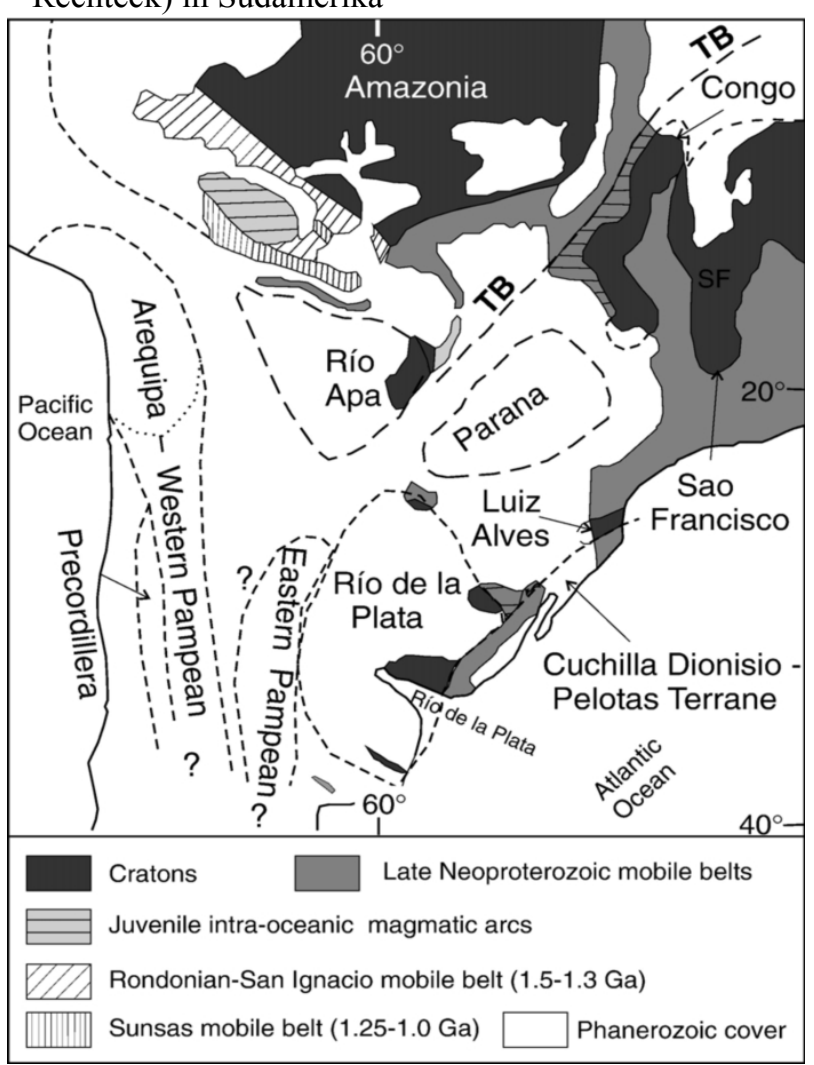

Abbildung 2: Übersicht über verschiedene Terranes in Südamerika. Die gestrichelten Linien geben die vermuteten Grenzen der Grenzen der Terranes an, da diese meistens unter Phanerozoischen Sedimenten liegen (verändert nach Kröner und Cordani 2003; Cordani et al. 2003 und Rapela et al. 2007).
Das untersuchte Arbeitsgebiet der östlichen Sierras Pampeanas befindet sich in Zentralbis Nordwest Argentinien (Abb. 1). Anhand der heute vorliegenden Verteilung anderer Kratone und Terranes in Südamerika kommen als mögliche Liefergebiete der heutigen Metasedimente der östlichen Sierras Pampeanas er östlich angrenzende Río de la Plata Kraton, die westlich gelegenen westlichen Sierras Pampeanas, sowie das sich nordwestlich befindende Arequipa-Antofalla Terrane, die nördlich gelegenen der Río Apa und Sunsas Kratone und das nordwestlich gelegene Parana Terrane (Abb. 2) in betracht. Zieht man nicht nur die heutige Verteilung der Terranes und Kratone Südamerikas, sondern auch die Paläogeographie in Betracht (Abb. 3), so kommen als weitere Quellen der Sedimente Bereiche östlich des Río de la Plata Kratons wie das Cuchilla Dionisio Pelotas Terrane und Bereiche aus dem südlichen Afrika wie der Kalahari Kraton in Frage. Eine Sedimentation könnte von Norden, Osten oder Süden stattgefunden haben (Abb. 3). Eine Provenienz aus den westlichen Sierras Pampeanas kann anhand der Paläogeographie ausgeschlossen werden, da sich dieses Terrane zum Zeitpunkt der Sedimentation zu weit entfernt befunden hat. Eine Schüttung von aus diesem Bereich an den Westrand Gondwanas ist daher auszuschließen.

Die östlichen Sierras Pampeanas bestehen 


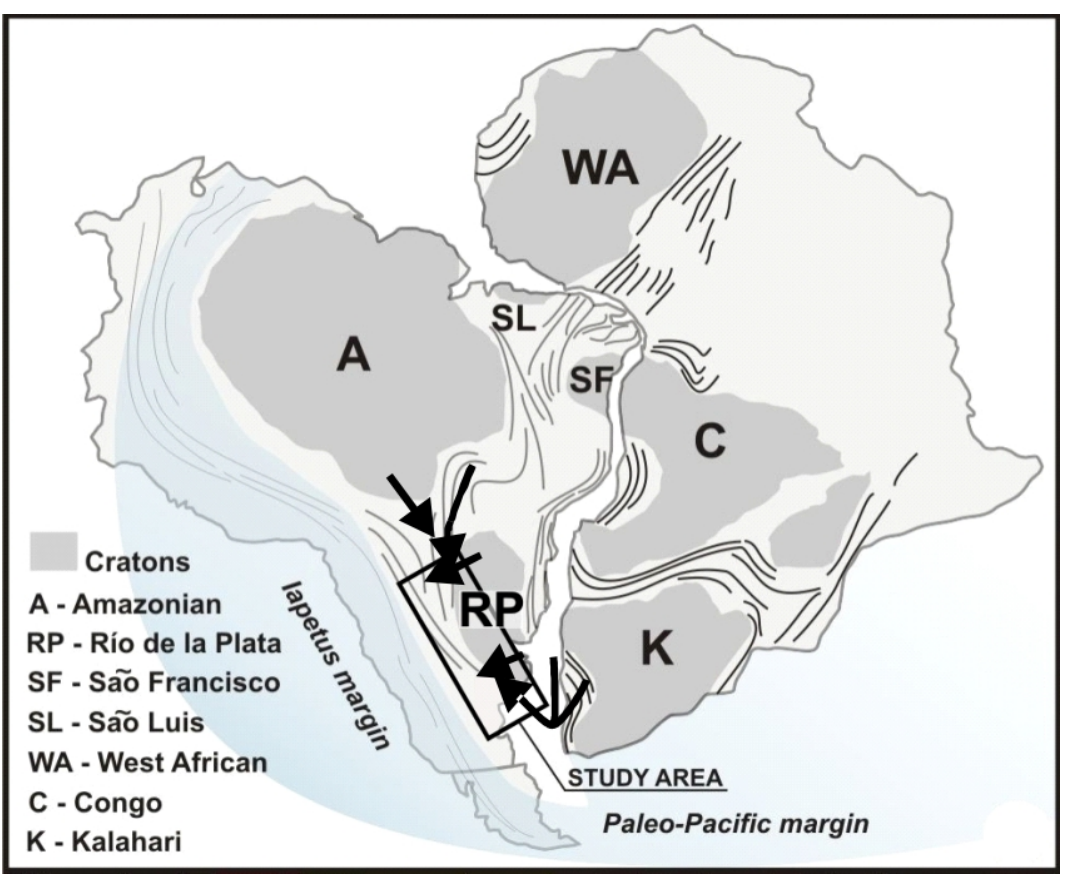

Abbildung 3: Paläogeographie von Westgondwana vor ca. $580 \mathrm{Ma}$. Die schwarzen Pfeile zeigen Schüttungsrichtungen möglicher Liefergebiete an (verändert nach Cingolani et al. submitted). aus verschiedenen kleineren Bereichen, die Miozän bis heute rezent gehoben wurden/werden. Ursache für diese Hebung ist die flache Subduktion der Nazca Platte unter die Südamerikanische Platte im Bereich von $27^{\circ}-$ 3330'S (Ramos et al. 2002). Der östliche Bereich dieses Gebietes ist als Pampeanes Orogen bekannt und wird durch spätneoproterozoische bis unterkambrische Sedimentation

sowie neoproterozoische bis kambrische Deformation, Magmatismus und Metamorphose charakterisiert (Rapela et al. 2007; Adams et al. 2008; Siegesmund et al. 2009). Der westliche Bereich wird durch die famatinische Orogenese geprägt (Abb. 4) und ist durch eine oberkambrische, marine Sedimentation und ordovizischen Magmatismus gekennzeichnet (Sims et al. 1998; Rapela 2000; Steenken et al. 2006; Collo et al. 2009). Im Norden, zwischen $22^{\circ}-$ $27^{\circ} \mathrm{S}$ wird das Pampeane Orogen durch die Puncoviscana Formation (Abb. 5) repräsentiert, die aus niedrig bis sehr niedrig gradigen Metasedimenten aufgebaut wird. Mögliche höhergradige Equivalente sind zwischen $27^{\circ}-33^{\circ} \mathrm{S}$ aufgeschlossen (Abb. 6), in Córdoba, La Rioja und San Luis, bis zum äußersten Süden der östlichen Sierras Pampeanas in der Provinz San Luis (Pankhurst et al. 1998; Rapela et al. 1998, 2007; Schwartz und Gromet 2004; Steenken et al. 2004; Zimmermann 2005, Schwartz et al. 2008).

Die Puncoviscana Formation Turner (1960) befindet sich im südlichsten Bereich Boliviens und im Nordwesten Argentiniens und erstreckt sich zwischen $22^{\circ} \mathrm{S}$ und $27^{\circ} \mathrm{S}$, bis zur Stadt Tucumán (Abb. 5). Die Aufschlüsse reichen über $800 \mathrm{~km} \mathrm{~N}-\mathrm{S}$ und bestehen aus niedrig- bis sehr niedriggradigen Metasedimenten, weitestgehend Tonsteine, Schiefer und Sandsteine, die mehrere 1000 m mächtig sind. Diese Einheit kommt in der Cordillera Oriental und in der östlich davon gelegenen Puna vor. Auf den siliziklastischen Flysch-Turbiditen und den pelagischen Tonsteinen wurden Spurenfossilien gefunden, die Oldhamnia und weiteren Lebewesen zugeordnet wurden. Aufgrund der Bestimmung der Spezies wurde ein 


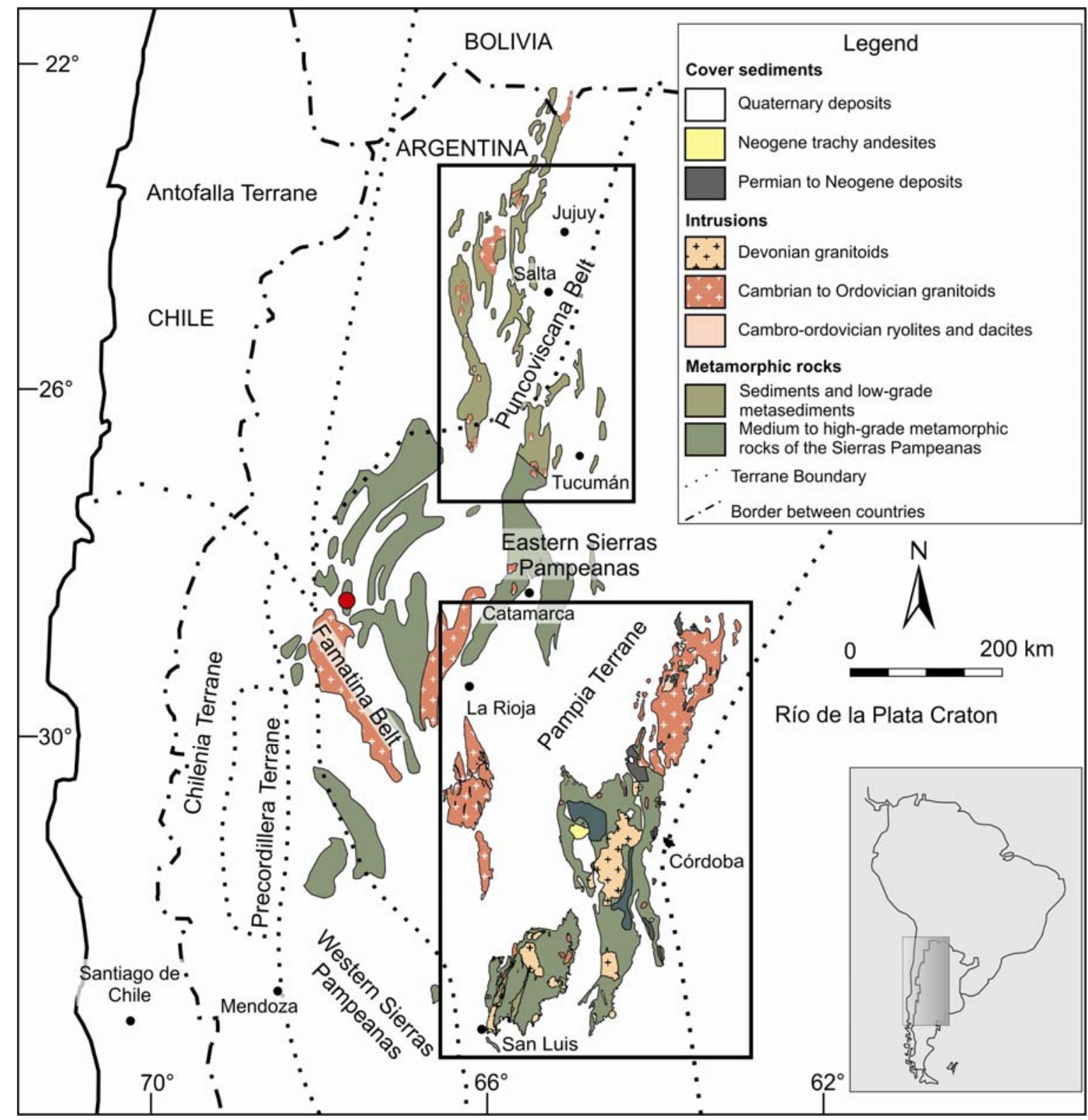

Abbildung 4: Geologische Skizze der östlichen Sierras Pampeanas, der Puncoviscana Formation und der wichtigsten Terranes westlich des Río de la Plata Kratons (verändert nach Lucassen et al. 2000; Rapela et al. 2007; Ramos 2008). Die Kästen rahmen die Untersuchungsgebiete ein. Der rote Punkt oberhalb der Beschriftung „Famatina Belt markiert die Neigro Peinado Formation und die Achavil Formation (Collo et al. 2009).

ediakarisches bis früh-kambrisches Sedimentationsalter bestimmt (Aceñolaza und Durand 1996; Aceñolaza und Tortello 2003). Ein Minimalalter der Sedimentation in der Puncoviscana Formation wurde durch das Intrusionsalter eines syn- bis postorogenen Kalkalkaligranites mit Hilfe von U-Pb Datierungen an Zirkon von Bachmann et al. (1987) auf $536 \pm 7$ Ma und $534 \pm$ 9 Ma bestimmt. Dies stimmt mit detritischen Zirkonen von Lork et al. (1990) überein, welche diese auf 570 - 540 Ma datierten und ein Maximalalter der Sedimentation anzeigen. Adams et al. (2006, 2008) datierten ebenfalls detritische Zirkone mittels der LA ICP-MS Methode, wobei zwei Maxima zwischen 760 - 580 Ma und 1100 - 860 Ma auffallen. Weitere Alter sind im Meso- und Paläoproterozoikum zu finden, jedoch bilden sie nur untergeordnete Maxima. 


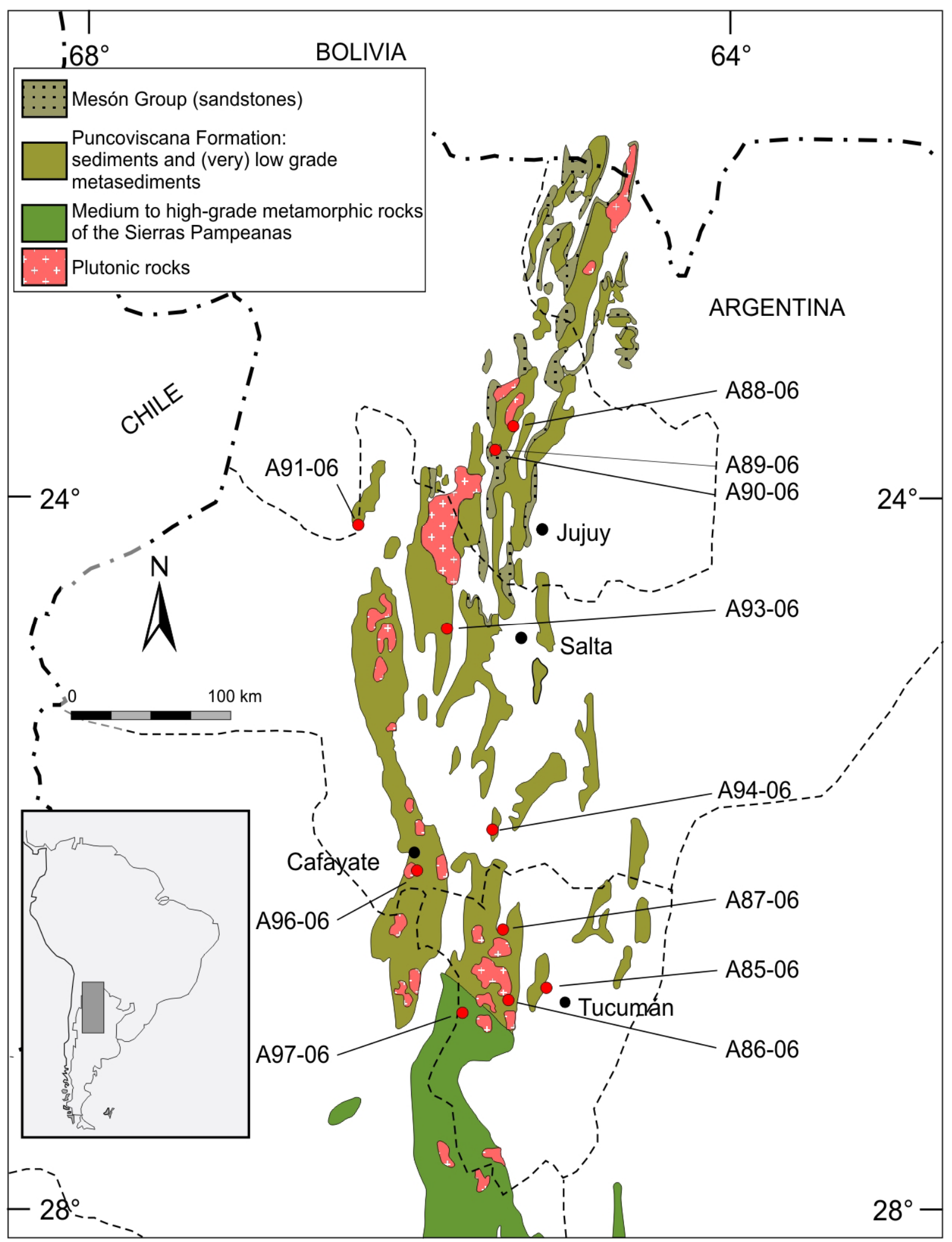

Abbildung 5: Geologische Karte der Puncoviscana Formation und der Mesón Group (verändert nach Aceñolaza und Aceñolaza, 2005). Die roten Punkte geben die Lokation der Proben an.

Allerdings fanden Adams et al. (2008) in einigen Proben ebenso jüngere Zirkone, die Maxima im Bereich von $550 \mathrm{Ma}$, aber auch noch jüngere zwischen 530 und $520 \mathrm{Ma}$ aufweisen. Diese Maxima stehen im Widerspruch zu den vorher genannten Maximal- und Minimalaltern der Sedimentation. In den Sierras de Chepes, Malanzán und Los Llanos (zur Vereinfachung im 
weiteren Verlauf lediglich Sierras de Chepes genannt) sind hauptsächlich granitoide Gesteine aufgeschlossen, die in fünf unterschiedliche Gruppen unterteilt werden können. Der Chepes Granodiorit ist die mit Abstand wichtigste Einheit der Sierras de Chepes (Abb. 6). Gesteine dieses Typs zeigen eine intensive Foliation an, die NW - SE streicht. Die weiteren, an der Oberfläche deutlich seltener aufgeschlossenen Granitoide sind der Chepes Porphyritic Granodiorit, der Asperezas Granit, der Tuaní Granit und der Tama Gabbro. Diese Gesteine sind bei Pankhurst et al. (1998) detailliert beschrieben. U-Pb Zirkonalter (Pankhurst et al. 1998) zeigen an, dass die Hauptphase der Intrusion des Chepes Granodiorits in das metasedimentäre Rahmengestein um $490 \pm 5$ Ma stattgefunden haben muss. Mit der Rb-Sr Methode wurde der Chepes Granodiorit auf $473 \pm 9$ Ma und der Asperezas Granit auf ein Alter um $470 \mathrm{Ma}$ datiert. Ein Alter ergibt lediglich $452 \pm 11 \mathrm{Ma}$, die Datenpunkte dieser Analyse streuen allerdings stark. Die unterschiedlichen $\mathrm{Rb}-\mathrm{Sr}$ Alter werden durch Geländebeobachtungen gestärkt, die ebenfalls für eine Platznahme des Chepes Granodiorits vor der Intrusion des Asperezas Granits sprechen. Kleinere posttektonische Monzogranite wurden ebenfalls mit Rb-Sr datiert und ergaben ähnliche Alter wie für die vorhergegangenen Körper um 470 Ma (Pankhurst et al. 1998). Die in den Sierras de Chepes aufgeschlossenen Metasedimente kommen unzusammenhängend, meist als in $\mathrm{N}-\mathrm{S}$ Richtung verlaufende Inseln vor. Pankhurst et al. (1998) beschrieben diese Schollen als zum Dachbereich gehörend. Die sehr niedrig bis amphibolitfaziellen Metasedimente sind häufig Metapelite mit Einschaltungen von Silt- und Sandsteinlagen. Einige der Aufschlüsse haben einen Durchmesser von lediglich $1 \mathrm{~m}$, andere sind allerdings deutlich größer und können mehrere $10 \mathrm{~m}$ lang sein. Die Protolithe dieser Gesteine wurden mit den südöstlich in den Sierras de Córdoba gelegenen Los Túneles Phylliten verglichen (Rapela et al. 1998). Die Metasedimente, in welche die verschiedenen Plutonite intrudiert sind, wurden ebenfalls mit der Rb-Sr Methode analysiert. Pankhurst et al. (1998) errechneten ein Alter einer Errorchrone von $513 \pm 31$ Ma. Auch wenn dieses Alter einen hohen Fehler aufweist, liegt es im Bereich einer weiteren $\mathrm{Rb}$-Sr Errorchrone, die ein Alter von $523 \pm 18$ Ma für die Los Túneles Phyllite aus den Sierras de Córdoba ergibt (Rapela et al. 1998).

Die Sm-Nd Analysen von Pankhurst et al. (1998) ergaben $\mathrm{T}_{\mathrm{DM}}$ Werte von 1,79 und 1,78 Ga mit dazugehörigen $\varepsilon \mathrm{Nd}_{(540)}$ Werten von -6,7 und -6,8 für die Metasedimente. Die granitoiden Gesteine ergaben $\mathrm{T}_{\mathrm{DM}}$ Alter zwischen 1,79 und 1,59 Ga und $\varepsilon \mathrm{Nd}_{(540)}$ Werte zwischen -3,7 und -6,2. Diese ähnlichen Alter und $\varepsilon \mathrm{Nd}_{(540)}$ Werte deuten auf S-Typ Granitoide mit geringem oder gar keinem Anteil an Mantelmaterial hin. 


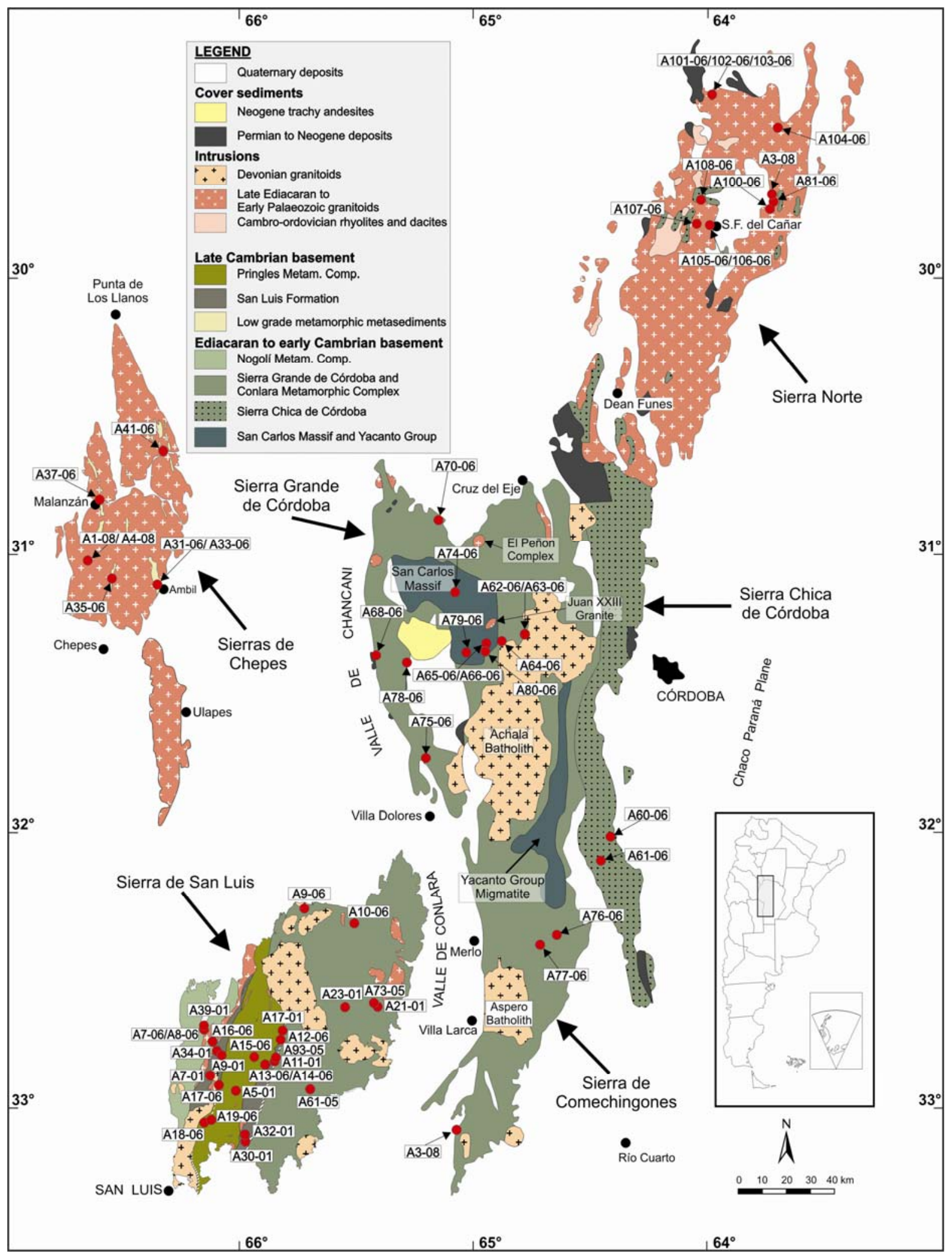

Abbildung 6: Geologische Karte der Sierra de San Luis, der Sierras de Chepes, der Sierras de Córdoba und der Sierra Norte (verändert nach Martino 2003; Steenken et al. 2006; Siegesmund et al. 2009). Die Probenlokationen sind durch rote Punkte dargestellt.

Die Sierras de Córdoba befinden sich östlich der Sierras de Chepes, sowie nördlich der Sierra de San Luis und bilden zusammen mit der Sierra Norte den östlichsten Bereich der östlichen Sierras Pampeanas. Dieses Gebiet besteht aus N-S streichenden Einheiten, die mehrfach 
metamorph überprägt worden sind (Abb. 6). Die vorkommenden Lithologien sind zumeist Ortho- und Paragneise, kambrische bis devonische Granitoide, Migmatite und teilweise (Ultra)Basite. Die Hauptgesteinsarten sind mittel- bis hochgradig amphibolitfazielle und granulitfazielle Migmatite, Gneise und Glimmerschiefer. Die einzige Ausnahme bilden die im äußersten Osten der Sierras de Córdoba anstehenden niedrigmetamorphen Los Túneles Phyllite. Die granulitfaziellen Gesteine können im Süden lokal aufgeschlossen sein, aber auch, wie beim San Carlos Migmatit, regional als diatexitische Migmatite ausgeprägt sein. Mutti et al. (1992) und Escayola et al. (1996) beschrieben die linsenförmig auftretenden (Ultra)Basite im westlichen Bereich als auseinander gerissene ophiolitische Einheit, was auf eine Obduktion ozeanischer Kruste hindeutet. Die hochgradigen Migmatite sind mit frühkarbonischen magmatischen Aktivitäten assoziiert. Diese Granitoide sind entweder rund, oder $\mathrm{N}-\mathrm{S}$ ausgelenkt und können maximale Durchmesser von mehreren Kilometern erreichen. Der Aluminium- Sättigungsindex (ASI) von Chappell und White (1992) zeigt an, dass es sich um S-Typ Granitoide handelt. Rapela et al. (1998) publizierten U-Pb Zirkonalter der stark peraluminösen S-Typ Granite um $530 \mathrm{Ma}$ und ein U-Pb Monazitalter eines Diatexites von $522 \pm 8$ Ma. Ein ähnliches U-Pb Monazitalter von $520 \pm 2$ Ma wurde von Gromet et al. (2005) berechnet. Dies macht deutlich, dass die Platznahme der Granitoide mit der Metamorphosespitze der Sierras de Córdoba zusammen fällt. Diese Körper könnten jedoch auch zwei Generationen angehören, denn Siegesmund et al. (2009) publizierten ein U-Pb Zirkonalter für einen Diatexit aus dem Süden der Sierras de Córdoba von 577 Ma. Zusätzlich fanden diese Autoren auch noch jüngere Alter im San Carlos Migmatit von $496 \pm 9$ Ma, die den vorherigen widersprechen. Ein solches jüngeres Alter könnte mit der HT/LP Metamorphose in der Sierra de San Luis (Hauzenberger et al. 2001; Steenken et al. 2006) in Zusammenhang stehen oder mit den Granitoidintrusionen der Sierras de Chepes in Verbindung gebracht werden (Pankhurst et al. 1998). Schwartz und Gromet (2004) datierten Zirkone aus Metasedimenten aus dem Nordwesten der Sierras de Córdoba mittels U-Pb Einzelkorndatierungen. Als Ergebnis erhielten sie ein Maximum zwischen 1050 und $950 \mathrm{Ma}$ und ein jüngeres zwischen 750 und 550 Ma. Diese Maxima konnten ebenso in Metasedimenten der Puncoviscana Formation (Adams et al. 2008) und im Conlara Metamorphic Complex in der Sierra de San Luis nachgewiesen werden (Steenken et al. 2006). Im zentralen Bereich der Sierras de Córdoba liegen der Achala Batholith und der Cerro Aspero Batholith. Diese beiden postorogenen Intrusionen wurden im mittleren bis späten Paläozoikum durch die Injektion granitoider Schmelze in die Kruste gebildet. Ursächlich hierfür ist die Achalische Orogenese, die durch die Kollision des Chilenia Terranes mit dem 
Westrand Gondwanas ausgelöst wurde (Ramos et al. 1986). Eine spät-devonische bis frühkarbonische zeitliche Einordnung ist durch eine Rb-Sr Isochrone von $358 \pm 9 \mathrm{Ma}$ (Rapela et al. 1990) möglich. Auch wenn ein Unterschied von ca. 20 Ma besteht, so ist dieses Alter dennoch mit dem U-Pb Zirkon Kristallisationsalter des Las Chacras Batholiths in der Sierra de San Luis zu vergleichen, das Siegesmund et al. (2004) auf $382 \pm 5$ Ma datierten.

Unmittelbar nordöstlich der Sierras de Córdoba befindet sich die Sierra Norte (Abb. 6). Kalkalkaline Granodiorite, Monzogranite und etwas jüngere Rhyolithe, Dacite und miarolitische Monzogranite prägen diese Sierra und machen ca. $95 \%$ der Aufschlüsse aus. Diese magmatischen Gesteine wurden als neoproterozoisch bis kambrisch datiert (Lira et al. 1997; Rapela et al. 1998; Schwartz et al. 2008). Dies entspricht dem Alter der meisten Intrusionen der Sierras de Córdoba, die im Bereich von 530 Ma liegen. Andere Autoren datierten die Effusiva jedoch als älter. So wurde ein Rhyodacit mittels U-Pb an Zirkonen auf $557 \pm 4 \mathrm{Ma}$ datiert (Söllner et al. 2000) und ein rhyolitischer Ingnimbrit auf 584 +22/-14 Ma (Llambias et al. 2003). Diese Alter stehen im Widerspruch zu den Beobachtungen von Lira et al. (1997), dass die Dacite, Rhyolithe und miarolitischen Monzogranite die Intrusivgesteine schneiden und durchschlagen, also jünger sein müssen. Das metasedimentäre Rahmengestein tritt nur untergeordnet auf. Die vorliegenden Metapsammite und Metapelite zeigen einen intrusiven Kontakt zu den Granodioriten und Monzograniten und weisen einen sehr geringen bis mittleren Metamorphosegrad auf (Lira et al. 1997). Diese Rahmengesteine kommen hauptsächlich im zentralen Bereich der Sierra um die Stadt San Francisco del Chañar und im Norden vor (Abb. 6).

Die südlichsten Aufschlüsse der östlichen Sierras Pampeanas sind in der Sierra de San Luis zu finden. Dieses Gebiet besteht aus drei NNE streichenden amphibolit- bis granulitfaziellen Komplexen, die von Ost nach West in den Nogolí Metamorphic Complex, den Pringles Metamorphic Complex und den Conlara Metamorphic Complex unterteilt werden. Östlich und westlich des Pringles Metamorphic Complex ist die San Luis Formation zu finden (Prozzi und Ramos 1988), die im Gegensatz zu den anderen Einheiten lediglich schwach metamorph ist.

Der Pringles Metamorphic Complex und der Conlara Metamorphic Complex werden hauptsächlich aus metasedimentären Gesteinen aufgebaut, wohingegen der Nogolí Metamorphic Complex hauptsächlich aus Orthogneisen besteht. Diese drei Einheiten wurden von ordovizischen und devonischen Granitoiden intrudiert. Die duktile Deformation und das kompressive geologische Regime hielten in diesem Gebiet bis ins Karbon an und wurden erst durch die Öffnung kontinentaler Becken beendet (Sims et al. 1998; Steenken et al. 2006; 
Collo et al. 2009). Im Pringles Metamorphic Complex sind (ultra)mafische Gesteine wie Norite, Gabbros, Dunite, Pyroxenite, Hornblendite und Amphibolite zu finden. Diese stehen mit einem Back-Arc Becken in Zusammenhang, das im Ordovizium (Famatinische Orogenese) geöffnet wurde und gehen mit lokal granulitfazieller Metamorphose der Metasedimente einher (Sims et al. 1997; Hauzenberger et al. 2001). Die Sierra de San Luis wurde in den letzten drei Jahrzehnten intensiv auf ihre strukturelle, metamorphe und magmatische Entwicklung untersucht (Kilmurray und Dalla Salda 1977; López de Luchi 1987, 1996; Ortiz Suárez et al. 1992; Llambias et al. 1998; Sims et al. 1998; von Gosen und Prozzi 1998; Delpino et al. 2001, 2007; González et al. 2002; López de Luchi et al. 2003, 2004, 2008; Steenken et al. 2004, 2006, 2008).

Die geodynamische Entwicklung und die zeitlichen Abfolgen des Conlara Metamorphic Complex auf der einen Seite und den westlich gelegenen Einheiten werden immer noch kontrovers diskutiert. Aufgrund der U-Pb SHRIMP Analyse detritischer Zirkone kann ein Maximalalter des Conlara Metamorphic Complex von $587 \pm 7$ Ma angegeben werden (Steenken et al. 2006). Siegesmund et al. (2009) dokumentierten ein PbSL Alter aus dem Conlara Metamorphic Complex von $564 \pm 21$ Ma, das die maximalen Metamorphosebedingungen anzeigt. Analysen mit der SHRIMP Methode lassen auf eine spätere Sedimentation des Pringles Metamorphic Complex um 530 Ma schließen (Sims et al. 1998; Steenken et al. 2006). Dieses Alter deckt sich mit einem konventionellen U-Pb Zirkonalter aus der San Luis Formation. In dieser Einheit wurde ein synsedimentärer Metavulkanit auf $529 \pm 12$ Ma datiert (Söllner et al. 2000), was dem Alter der Sedimentation entspricht. Als Minimalalter des Pringles Metamorphic Complex können metamorph gewachsene Ränder an Zirkonen angesehen werden, die Steenken et al. (2006) auf $498 \pm 10$ Ma datierten. Im Nogolí Metamorphic Complex können mesoproterozoische oder noch höhere Sedimentationsalter durch das Vorkommen von gebänderten Eisenerzen und Spinifex - Strukturen in Komatiiten angesehen werden (Sato et al. 2001; Gonzalez et al. 2002). Allerdings werfen neuere SHRIMP Zirkondatierungen der Komatiite Zweifel an ihrem hohen Kristallisationsalter auf (Sato et al. 2006). Steenken et al. (2006) konnten im Nogolí Metamorphic Complex überhaupt keine prä-pampeanen Alter nachweisen. Die datierten Monazite zeigten lediglich Alter zwischen 484 Ma und 473 Ma. Dies ist im Pringles Metamorphic Complex ähnlich. Die Datierungen von Metasedimenten der gleichen Autoren zielen in die gleiche Richtung, denn auch hier konnten keine mesoproterozoischen Alter nachgewiesen werden. Vielmehr zeigten ihre U-Pb Zirkondaten ein konkordantes pampeanes Maximum bei 545 Ma und eine famatinische Überprägung bei 498 Ma an. Im El Peñon 
Batholith, der sich im Conlara Metamorphic Complex befindet, kommen Xenolithe der bereits verfalteten gebänderten Schiefer vor. Dies bedeutet, dass mindestens eine Deformation des Conlara Metamorphic Complex bereits vor der Intrusion des Plutons stattgefunden haben muss. Das Alter des el Peñon Batholiths auf $497 \pm 8$ Ma datiert, was zeitlich mit der fast isobaren HT-Metamorphose des Pringles Metamorphic Complex übereinstimmt (Steenken et al. 2006).

Einige Autoren sehen in der San Luis Formation das Äquivalent zur Puncoviscana Formation (Toselli 1990; Willner 1990; Rapela et al. 1998; Söllner et al. 2000), andere wiederum zeigten anhand geochemischer und isotopengeochemischer Daten sowie Altersdatierungen Ähnlichkeiten zwischen dem Conlara Metamorphic Complex und der Puncoviscana Formation (Steenken et al. 2004, 2006; Zimmermann 2005). Schwartz und Gromet (2004) datierten Metasedimente aus dem Nordwesten der Sierras de Córdoba und der Puncoviscana Formation und zogen anhand vergleichbarer Altersverteilungen detritischer Zirkone und vergleichbarer Isotopendaten den Schluss, dass die Sierras de Córdoba ein höhergradiges Äquivalent der Puncoviscana Formation sein könnte.

Einige Autoren wie Leite et al. (2000) und Janesi et al. (2001) sind der Meinung, dass die pampeane Orogenese am Westrand des Río de la Plata Kratons ein Teil der Panafrikanischen - Brasiliano Orogenese ist. Die pampeane Orogenese, die zwischen 570 und 510 Ma stattfand (Ramos 1988; Rapela et al. 1998, 2007; Siegesmund et al. 2009) wurde durch die tektonische Platznahme des pampeanen Terranes am Südwestrand des Río de la Plata Kratons ausgelöst und kontrolliert. Die durchgehende, ostwärts gerichtete Subduktion am Westrand des neu geformten Kontinentalrandes im Kambrium und die folgende Verschmelzung mit dem Cuyania/Precordillera Terrane führte zur famatinischen Orogenese (Ramos et al. 1986; Sims et al. 1998), die mit lokaler kambischer Sedimentation in Verbindung steht (Steenken et al. 2006).

Für die pampeane Orogenese wurden mehrere geodynamische Modelle entwickelt. Einige gehen von einer Kollision aus, andere beschreiben die Vorgänge ohne die Kollision eines Terranes oder Kratons. Durch die verschiedenen Modelle wurden unterschiedliche Ablagerungsbedingungen der Protolithe der östlichen Sierras Pampeanas in die Literatur eingeführt. Aufgrund der (ultra)mafischen Körper wurde eine auseinander gerissene ophiolitische Sequenz diskutiert, welche das Vorhandensein von ozeanischer Lithosphäre am Kontinentalrand zur Folge hat (Mutti et al. 1987; Escayola et al. 1996, 2007). Modelle, die eine Kollision befürworten, gehen davon aus, dass die unterschiedlichen metasedimentären Einheiten an einem passiven Kontinentalrand abgelagert wurden, der später zu einem aktiven 
wurde. Rapela et al. (1990) gingen von einem passiven Kontinentalrand im Westen des Río de la Plata Kratons aus, an dem die Protolithe abgelagert wurden. Andere Arbeiten wie von Ramos (2008) bevorzugen die Sedimentation der Klastika in einem Aulakogen. Ein Tripelpunkt zwischen dem Arequipa-Antofalla, Amozina und Río Apas Kraton würde demnach die unterschiedlichen sedimentären Fazies erklären. Ein allochthones bis paraallochthones Modell wurde von Rapela et al. (1998) befürwortet. Eine nach Osten gerichtete Subduktion ging demnach mit kalk-alkalinem Magmatismus in der Sierra Norte einher und wurde durch die Kollision des pampeanen Terranes beendet. Eine weiter Möglichkeit ist die Überschiebung eines passiven Kontinentalrandes östlich des Río de la Plata Kratons. Die auch nach den Überschiebungen weiter verlaufenden magmatischen Aktivitäten, die durch felsische, kalk-alkaline Granitoide in der Sierra Norte charakterisiert sind, haben ihren Ursprung in einem post-kollisionalen orogenen Kollaps nach der Kollision und der Aufschmelzung älterer I-Typ Gesteine (Sims et al. 1998; Rapela et al. 1998). Ramos (2008 und darin enthaltene Referenzen) nimmt an, dass das Becken, in welches die Protolithe der Puncoviscana Formation abgelagert wurden, ein randliches Vorlandbecken ist, das durch die Kollision des Pampia Blocks an der Seite der östlichen Sierras Pampeanas mit dem Río de la Plata Kraton entstanden ist. Ein nicht kollisionales Szenario wurde von Schwartz et al. (2008) präsentiert. In diesem Modell wird die Kollision eines ozeanischen Rückens mit der Tiefseerinne beschrieben, da dieses viele Strukturen der pampeanen Orogenese wie lokale dextrale Scherzonen nach der magmatischen Aktivität am besten erklären kann. Die Plattengrenze änderte sich aufgrund der Kollision von einer konvergenten Grenze mit Subduktionszone zu einer Transformstörung. Rapela et al. (2007) und Casquet et al. (2008) gehen bei der Puncoviscana Formation von einer Einheit aus, die im Fore-Arc Bereich abgelagert wurde, allerdings nicht östlich des Río de la Plata Kratons, sondern südlich und westlich des Kalahari Kratons. Durch dextrale Scherzonen konnten die Metasedimente entlang des Westrandes des Río de la Plata Kratons an ihre heutige Position gebracht werden. Durch die Kollision der westlichen Sierras Pampeanas, ein Bereich des Amazonas und Arequipa Kratons, wurde die Puncoviscana Formation schließlich mit dem Río de la Plata Kraton verschweißt.

Um Hinweise auf die Provenienz der Puncoviscana Formation zu erhalten, wurde diese mit Haupt- und Spurenelementen, sedimentologischen Studien sowie anhand von Isotopenverhältnissen untersucht. In mehreren Arbeiten wurde ein passiver Kontinentalrand westlich des Río de la Plata Kratons beschrieben, bei dem die Sedimente als Turbidite abgelagert wurden (Aceñolaza et al. 1983; Willner et al. 1985; Ježek und Miller 1986; Rossi 
et al. 1997; DoCampo und Guevara 2002). Andere Autoren konzentrierten sich auf die Analyse vulkanischer Gesteine und schlossen daraus einen Wandel des geotektonischen Rahmens von einem Rift hin zu einem Back-Arc (Omarini et al. 1999). Kraemer et al. (1995) sowie Keppie und Bahlburg (1999) sprachen sich für eine Sedimentation in ein VorlandBecken aus. Dies wurde von Zimmermann (2005) bestätigt, der eine sehr große Anzahl von (Meta)Sedimenten geochemisch untersuchte $(\mathrm{n}=119)$. Ein starker vulkanischer Eintrag in die (Meta)Sedimente wurde von Bock et al. (2000) aufgrund der Nd- und Pb-Isotopie ausgeschlossen.

Obwohl sich die Puncoviscana Formation unmittelbar westlich des Río de la Plata Kratons befindet, ist eine ausschließliche Provenienz der klastischen Gesteine von diesem unwahrscheinlich, da die Alter der detritischen Zirkone des Río de la Plata Kratons Maxima bei über $2 \mathrm{Ga}$ aufweisen und diese Alter in den östlichen Sierras Pampeanas nur untergeordnet zu finden sind. Außerdem sind die Nd Modellalter zwischen 1.8 und $1.6 \mathrm{Ga}$ deutlich unterschiedlich zu denen des Río de Plata Kratons, die dort zwischen 2.8 und $2.2 \mathrm{Ga}$ liegen (Hartmann et al. 2002; Pankhurst et al. 2003; Rapela et al. 2007). Dies gilt für die gesamten östlichen Sierras Pampeanas (Bock et al. 2000; Schwartz und Gromet 2004; Steenken et al. 2004, 2006, 2008; Adams et al. 2006, 2008; Escayola et al. 2007; Rapela et al. 2007) und spricht gegen die Argumentation von Rapela et al. (1998). Der Unterschied zu den $\mathrm{T}_{\mathrm{DM}}$ Altern des Río de la Plata Kratons und die unterschiedlichen Zirkonalter sind zu groß. Ein mögliches Liefergebiet der Gesteine der Puncoviscana Formation könnte sich nordwestlich der heutigen Position in Gesteinen befinden, die von der Brasiliano Orogenese geprägt wurden (Brito Neves et al. 1999). Escayola et al. (2007) brachten eine weitere Möglichkeit in die Diskussion ein, bei der ein intra-ozeanischer Inselbogen zwischen dem Río de la Plata Kraton und dem pampeanen Terrane eine Sedimentzufuhr des paläoproterozoischen Materials in die (Meta)Sedimente der östlichen Sierras Pampeanas verhindert hat. Eine westgerichtete Sedimentation an einem aktiven Kontinentalrand wird auch von Zimmermann (2005) anhand geochemischer Daten favorisiert. Der Eintrag von Material des Río de la Plata Kratons wäre nach diesem Modell von dem magmatischen Bogen blockiert worden, ähnlich wie es heutzutage durch die Anden geschieht. Rapela et al. (2007) und Casquet et al. (2008) bevorzugten ebenfalls die Sedimentation in ein Fore-Arc Becken, jedoch gehen sie davon aus, dass die Sedimente nicht autochthon abgelagert wurden, sondern südlich und westlich des Kalahari Kratons und erst nachträglich anhand von dextralen Blattverschiebungen in ihre heutige Position gebracht wurden. Um dieses Modell zu testen, 
müssten die Gesteine des Kalahari Kratons detailliert dahingehend untersucht werden, ob sie mit den (Meta)Sedimenten der östlichen Sierras Pampeanas vergleichbar sind 


\section{Beschreibung des Probenmaterials}

Insgesamt wurden 66 Metasedimentproben des Nogolí Metamorphic Complex, des Pringles Metamorphic Complex, des Conlara Metamorphic Complex, der San Luis Formation, der Sierras de Chepes, der Sierras de Córdoba, der Sierra Norte und der Puncoviscana Formation auf Haupt- und Spurenelemente mittels RFA und ICP-MS analysiert. Von diesen Proben wurden 45 auf ihre Sm-Nd-Isotopie untersucht und bei 43 Proben wurde die Pb-Isotopie analysiert. Aus acht Proben wurden Zirkone separiert und mittels der SHRIMP Technik datiert. Die Probenlokationen sind auf den Abbildungen 2 und 3 eingezeichnet. Die Einteilung der Gesteine in Psammite oder Pelite erfolgte auf der Basis der Klassifikation von Herron (1988) und ist in Abbildung 4 dargestellt.

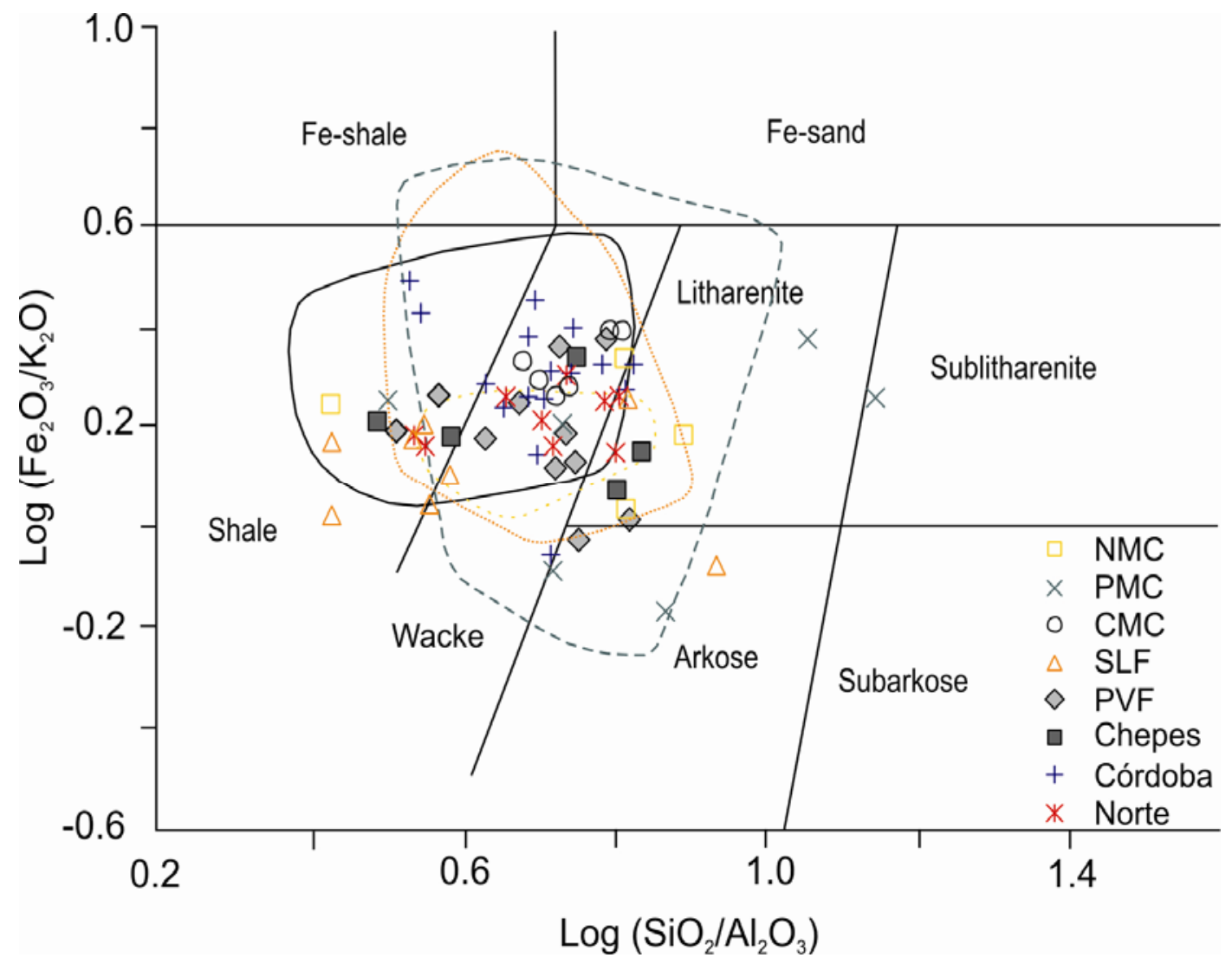

Abbildung 4: Chemische Klassifizierung der (meta)sedimentären Proben der östlichen Sierras Pampeanas und der Puncoviscana Formation der Cordillera Oriental, basierend auf $\log \left(\mathrm{Fe}_{2} \mathrm{O}_{3 \text { total }} / \mathrm{K}_{2} \mathrm{O}\right)$ vs. $\log \left(\mathrm{SiO}_{2} / \mathrm{Al}_{2} \mathrm{O}_{3}\right)$ nach Herron (1988). Die Felder beziehen sich auf die Verteilung von Literaturwerten von Sims et al. (1998), Brogioni (2001), López de Luchi et al. (2003) und Zimmermann (2005). Lang gestrichelte Linie: Pringles Metamorphic Complex, durchgehende Linie: Conlara Metamorphic Complex, gepunktete Linie: San Luis Formation, kurz gestrichelte Linie: Puncoviscana Formation. Soweit möglich wurden die Felder in den Farben dargestellt, welche die Symbole der jeweiligen Einheiten besitzen. Probe A102-06 liegt aufgrund eines hohen $\log \left(\mathrm{Fe}_{2} \mathrm{O}_{3 \text { total }} / \mathrm{K}_{2} \mathrm{O}\right)$ Wertes weit außerhalb des Diagramms. 


\subsection{Nogolí Metamorphic Complex}

Der Nogolí Metamorphic Complex besteht hauptsächlich aus felsischen Orthogneisen. Mafische Gneise kommen ebenso wie Paragneise nur untergeordnet vor. Die aus dieser Einheit genommenen Proben sind nach der Klassifikation von Herron (1988) in drei Fällen Litharenite (A7-06, A8-06 und A16-06) und einem Fall ein Tonstein (A39-01). Dabei wurde darauf geachtet, dass die Proben keine Anzeichen einer Migmatisierung oder Gänge enthielten. Die genommenen Proben stammen aus dem Nordosten des Nogolí Metamorphic Complex. In allen Proben ist die Mineralzusammensetzung Quarz, Plagioklas, Biotit, Muskovit mit akzessorischen Mineralen wie Zirkon, Apatit \pm Klinozoisit \pm Sillimanit. Durch die Ausrichtung der Biotite sind zwei Foliationen zu erkennen. Die metamorphen Maxima können in einigen Fällen durch das Wachstum von Silliamnit auf Kosten von Biotit im Dünnschliff erkannt werden (A16-06). Muskovit kann regellos vorkommen, was auf ein späteres Ereignis ohne gerichtete Deformation hindeutet (von Goosen und Prozzi 1998).

\subsection{Pringles Metamorphic Complex}

Der Pringles Metamorphic Complex besteht im Wesentlichen aus Paragneisen, Orthogneisen, Glimmerschiefern und seltener vorkommenden Amphiboliten. (Ultra)mafische Gesteine sind in einem Gürtel im westlichen Bereich dieser Einheit zu finden und stehen sowohl räumlich, wie auch zeitlich in Verbindung zu den granulitfaziellen Gesteinen (Hauzenberg et al. 2001; Steenken et al. 2007).

Aus diesem Gebiet wurden sechs Proben genommen. Bei einem Gestein handelt es sich um einen Tonstein (A15-06), eine Probe liegt im Feld für Wacke (A5-01), zwei wurden als Arkosen klassifiziert (A14-06 und A18-06) und bei zwei Proben handelt es sich um Litharenite bzw. Sublitharenite (A19-06 und A13-06). Im Gelände wurden die Gesteine als Metapsammite (A13-06, A14-06 und A19-06), Metapelit (A15-06) und Metapsammopelit (A5-01 und A18-06) angesprochen. Die Metapsammite A13-06 und A14-06 wurden im Osten dieser Einheit genommen und die Probe A19-06 im Südwesten. Bei diesen Metapsammiten ist eine Wechsellagerung von grobkörnigen und feinkörnigen Lagen zu erkennen. Bei den Gesteinen handelt es sich um Biotit-Muskovit Gneise, die aus Quarz, Plagioklas, Biotit, und Muskovit bestehen. Akzessorisch treten Zirkon, Apatit, \pm Granat, \pm Turmalin, \pm Titanit auf. Die Metapsammopelite wurden im Südwesten (A18-06) und im Zentrum des Pringles Metamorphic Complex genommen (A5-01). Bei diesen Gesteinen ist eine Wechsellagerung 
von pelitischem und psammitischem Material zu beobachten. Diese Lagerung ist geschert, sodass die einzelnen Bereiche als Linsen oder unterbrochene Lagen auftreten. Die psammitischen Bereiche sind stark angereichert an Quarz und enthalten etwas Plagioklas. Innerhalb dieser Lagen treten ausgelängte Biotit- und Muskovitkristalle auf, die ungleichmäßig verteilt sind. Die pelitischen Bereiche bestehen aus stark gebogenem Muskovit und Biotit, die ein S-C Gefüge definieren. Die metapelitische Probe A15-06 stammt aus dem Zentrum dieses Gebiets. Es handelt sich um graues Gestein, das aus Biotit, Quarz, Kalifeldspat, Plagioklas und Sillimanit besteht. Akzessorische Gemengteile sind Zirkon, Apatit und opake Minerale. Glimmer und Sillimanit kommen oft in gescherten Lagen vor und sind rotiert, wobei sie eine Wechsellagerung mit den Quarzlagen bilden.

\subsection{Conlara Metamorphic Complex}

Dieser Bereich besteht im Wesentlichen aus Paragneisen und Orthogneisen. Die Metasedimente werden durch Metagrauwacken dominiert, metapelitische Gesteine kommen weniger häufig vor. Untergeordnet treten Turmalinschiefer und Turmalinite auf. Alle hier genommenen Proben sind Wacken. Charakteristisch an den Gesteinen ist eine Wechselfolge von mittelkörnigen Lagen aus Quarz und Plagioklas sowie feinkörnigen Lagen, die von Biotit dominiert werden (A21-01, A23-01, A61-05 und A10-06). Die etwas höher metamorphen, sich oberhalb des Biotit Isograds befindenden gebänderten Gneise (A21-01, A23-01 und A1006), die ausführlich bei López de Luchi (1986) beschrieben sind, haben einen charakteristischen metamorphen Lagenbau, der durch den Wechsel von quarzdominierten und glimmerreichen Abschnitten auffällt. Die Glimmerlagen bestehen aus grünem Biotit, Plagioklas und etwas Quarz, sowie akzessorisch aus opaken Mineralen, Apatit und Zirkon. Die quarzreichen Lagen bestehen aus Quarz, Plagioklas und etwas Biotit. Außerdem kann in manchen Bereichen reliktischer Granat zu finden sein.

\subsection{San Luis Formation}

Diese Einheit besteht hauptsächlich aus Phylliten. Schwarze Tonsteine sind nur untergeordnet zu finden und Konglomerate sind selten. Sechs von acht Proben dieser Einheit liegen in oder sehr nah an dem Feld für Tonsteine (A7-01, A11-01, A17-01, A30-01, A32-01, A34-01), bei einer Probe handelt sich um einen Litharenit (A9-01) und eine Probe liegt im Feld für Arkosen (A12-06). Im Gelände wurden die Arkose und der Litharenit als feinkörnige 
Metapsammite angesprochen, die Tonsteine als sehr Phyllite. Die Zusammensetzung der Metapsammite ist Quarz, Plagioklas, Muskovit und Biotit, wobei die Glimmer die Foliation definieren. Weder bei den Plagioklas-, noch bei den Quarzkörnern sind Effekte einer dynamischen Rekristallisation zu erkennen, allerdings ist bei vielen Quarzkörnern eine statische Rekristallisation auszumachen, die durch eine Vielzahl von Tripelpunkten zu erkennen ist. Bei den Metapeliten bzw. Phylliten ist eine Wechselfolge von feinkörnigen Glimmern und grobkörnigeren, quarzreichen Lagen mit einer feinkörnigen Matrix auffällig. Außerdem sind einzelne größere Quarzkristalle charakteristisch, die in der Foliation dynamisch rekristallisiert und wahllos in dem Gestein verteilt sind.

\subsection{Puncoviscana Formation}

Diese Einheit besteht im nördlichen Bereich aus sehr niedrig- bis niedriggradigen und im Süden aus mittelgradigen Metasedimenen. Bei den genommenen Proben handelt es sich um zwei Tonsteine (A85-06 und A86-06), sieben Wacken (A88-06, A89-06, A91-06, A93-06, A94-06, A96-06 und A97-06) und zwei Proben, die im Bereich zwischen Litharenit und Arkose zu finden sind (A87-06 und A90-06). Bei den Proben A85-06 und A86-06 handelt es sich um sehr niedriggradig metamorphe Tonsteine, sieben Proben wurden im Gelände als niedrig metamorphe Psammite (A87-06, A88-06, A89-06 A90-06, A91-06, A93-06 und A9406) angesprochen und bei zwei Proben handelt sich um mittelgradig metamorphe Psammite (A96-06, A97-06). Die Metapelite sind grünlich bis gräulich und haben einen hohen Anteil an Serizit, Hellglimmern und Chlorit. In dieser Matrix befinden sich in unterschiedlicher Häufigkeit moderat gerundete Quarzkörner und einige Plagioklase und Muskovite. In einigen Bereichen sind diese Klasten selten, in anderen kommen siltige Lagen dieser Minerale vor. In Probe A85-06 sind kleine Mikrorisse zu erkennen. Anhand dieser Wegsamkeiten sind Fluide in das Gestein eingedrungen, die eine bräunliche Oxidation der in der Nähe des Risses befindlichen Bereiche verursacht haben. Die Metapsammite (A87-06, A88-06, A89-06 A9006, A91-06, A93-06 und A94-06) enthalten als Hauptgemengteile Quarz, Plagioklas, Muskovit \pm Kalzit. Diese Körner sind von Hellglimmern und Chlorit umgeben. Kalzit kann nicht nur als primärer Bestandteil des Gesteins auftreten, sondern bildet auch monomineralische sekundäre Gänge. Es wurde darauf geachtet, solche Gänge bei der Probenpräparation zu entfernen, um die sekundären Einflüsse auf die Analyse möglichst gering zu halten. Bei den beiden mittelgradig metamorphen Psammiten A96-06 und A97-06 handelt es sich um gebänderte Gneise, die denen aus dem Conlara Metamorphic Complex 
sehr ähnlich sind. Die Bänderung besteht aus einer Wechsellagerung von quarzreichen und glimmerreichen Lagen. In der Probe A97-06 konnten zwei verschiedene Biotite identifiziert werden. Eine Generation ist plattig und braun, die andere ist faserig und grünlich. Nur in dieser Probe tritt Granat in den grobkörnigen Lagen auf.

\subsection{Sierras de Chepes}

Bei den Proben aus den Sierras de Chepes handelt es sich um zwei Tonsteine (A31-06 und A37-06), eine Wacke (A41-06) und zwei Litharenite (A33-06 und A35-06). Die Proben wurden unmittelbar nordöstlich der Stadt Ambil genommen (A35-06 und A33-06), im zentralen Bereich der Sierra de Chepes (A35-06), direkt nordwestlich der Stadt Malanzán und westlich der Stadt Olta (A41-06). Die Proben A31-06 und A37-06 wurden im Gelände als Phyllite angesprochen. Im Dünnschliff zeigte sich eine Wechsellagerung von feinkörnigen Quarz-Plagioklas Lagen und sehr feinkörnigen Glimmerlagen. Dies konnte in beiden Proben beobachtet werden, allerdings ist A31-06 generell etwas grobkörniger als A37-06. Makroskopisch scheinen die Gesteine gering metamorph zu sein. Im Dünnschliff ist das Wachstum von Biotit zu erkennen, sodass dieses Mineral als typisch für die metamorphe Fazies angesehen wird. Die Wacke A41-06 hat eine ähnliche Korngröße wie Probe A31-06. Auch die Mineralogie ist ähnlich, allerdings kommen weniger Glimmer vor und diese Probe zeigt weder eine Bänderung noch einen sedimentären Lagenbau. Es handelt sich um ein homogenes, niedriggradiges Gestein. Die Proben A33-06 und A35-06 sind nach dem Diagramm von Herron (1988) Litharenite. Makroskopisch handelt es sich um psammitische Gesteine, wobei A33-06 feinkörniger als A35-06 ist. Die letztgenannte Probe ist gebändert und diese Bänderung wiederum verfaltet. Eine Bänderung ist auch bei A33-06 vorhanden, allerdings auch mikroskopisch nur schwer nachweisbar. Beide Proben weisen wie auch A3106 und A37-06 eine Metamorphose oberhalb der Biotitisograde auf.

Zwei weitere Proben wurden zur SHRIMP Datierung detritischer Zirkone genommen (A1-08 und A4-08). Bei beiden handelt es sich um typische gebänderte Gneise. Sowohl der Metamorphosegrad, die Korngröße als auch die Bänderung sind mit der Probe A35-06 vergleichbar. Im Dünnschliff selber ist keine Faltung der Bänderung zu erkennen, im Handstück ist dies jedoch deutlich sichtbar. 


\subsection{Sierras de Córdoba}

Das Probematerial der Sierras de Córdoba muss zunächst in drei verschiedene Gruppen unterteilt werden. Die erste Gruppe schließt niedrig- bis mittelgradig metamorphe Gesteine ein und umfasst die Proben A62-06, A63-06, A64-06, A65-06, A66-06, A68-06, A70-06 und A75-06. Die zweite Gruppe besteht aus hochgradig metamorphen Diatexiten. Zu dieser Gruppe gehören die Proben A60-06, A61-06, A74-06, A76-06, A79-06 und A80-06. Zur dritten Gruppe gehören die Proben A77-06 und A78-06. Bei diesen handelt es sich um hochgradig metamorphe gebänderte Gneise. Nach der Nomenklatur von Herron (1988) sind lediglich zwei der insgesamt 16 Proben als Tonsteine anzusprechen und zwar die metasedimentäre Probe A65-06 und der Diatexit A76-06. Alle anderen Gesteine liegen im Diagramm im Bereich des Felds für Wacken.

Bei der als Tonstein ermittelten Probe A65-06 handelt es sich um eine gneisige Enklave in einem Diatexit. Der Einfluss der diese Probe umgebenden Schmelze auf diese Enklave ist unklar. Die Probe weist einen hohen Anteil an Biotit und Granat auf, ähnlich wie der zweite Tonstein A76-06. Hauptgemengteile sind Quarz, Plagioklas, Biotit und Granat. Kleinere Plagioklaskörner sind oft retrograd in Serizit umgewandelt. Die Granatkörner sind von feinkörnigen Glimmern umgeben. Dies ist auch in Rissen zu beobachten, welche die Granate durchziehen. Der Unterschied der beiden Proben ist, dass es sich bei A76-06 nicht um eine Enklave handelt, sondern um einen Diatexit, der im Gelände als Tonalit angesprochen wurde. Obwohl es sich bei allen anderen Gesteinen nach der Nomenklatur von Herron (1988) um Wacken handelt, treten aufgrund der unterschiedlichen Metamorphosebedingungen sehr starke Unterschiede auf. Die Proben A62-06 und A63-06 wurden nordwestlich des Achala Batholiths genommen. Es handelt sich um Gneise, die einen Lagenbau aus feinkörnigen und mittelkörnigen Lagen aufweisen. Der Unterschied dieser Lagen ist nicht nur die Korngröße. Die feinkörnigen Lagen weisen einen höheren Glimmergehalt auf, wohingegen die gröberen Lagen quarzdominiert sind. Hauptgemengteile sind Quarz, Biotit, Muskovit und Plagioklas. Muskovit kommt nicht nur foliationsparallel vor, sondern kann auch nach Ausbildung der Foliation gewachsen sein. In diesen Fällen schneidet er die Foliation. Beide Gesteine zeigen eine Bänderung, allerdings ist nicht zweifelsfrei zu klären, ob diese auf einen Materialwechsel während der Sedimentation zurückgeht oder ob es sich um eine metamorphe Bänderung auf Grund von Drucklösungen handelt. Die Probe A64-06 wurde zwischen dem Achala Batholith und dem San Carlos Massiv genommen und im Gelände als Psammopelit angesprochen. Bei dieser Probe liegt eine Wechsellagerung von Quarz-Plagioklas und Biotitlagen vor. Die Probe 
A66-06 wurde direkt neben der als Tonstein plottenden Probe A65-06 genommen. Auch diese Probe wurde als gneisige Enklave aus dem Diatexit genommen, allerdings war die Enklave wesentlich größer als bei A65-06. Der Mineralbestand ist Quarz, Plagioklas, Biotit und Granat. Auch bei dieser Probe ist eine leichte Bänderung zu erkennen, die ebenfalls durch Quarz-Plagioklas und Biotit Lagen gebildet wird. Die Probe A68-06 wurde im Bereich von Los Túneles genommen. Makroskopisch handelt es sich um ein feinkörniges Gestein mit einem hohen Anteil an Phyllosilikaten auf den Schichtflächen. Im Dünnschliff ist zu erkennen, dass Glimmer, vor allem aber Chlorit die Foliation bildet, Quarz allerdings der Hauptbestandteil dieser Probe ist. Bei diesem Gestein handelt es sich um einen Siltstein, der grünschieferfaziell überprägt wurde. Die Proben A70-06 und A75-06 sind beide gebänderte Gneise, die sich bei der Metamorphose oberhalb der Biotitisograde befunden haben. Mikroskopisch ist die Bänderung aufgrund des großen Abstandes der einzelnen Bereiche schlecht zu erkennen, makroskopisch ist sie jedoch hervorragend ausgebildet. Diese gebänderten Gneise sind denen im Conlara Metamorphic Complex in der Sierra de San Luis ähnlich. Bei den Proben A77-06 und A78-06 handelt es sich ebenfalls um gebänderte Gneise, allerdings lagen in diesen beiden Fällen höhere Metamorphosebedingungen vor. Zwischen den Quarz-Plagioklas - Lagen und den Biotit - Lagen ist bei diesen Proben meistens ein Bereich aus Sillimanit zu beobachten, der bei den niedriggradigen gebänderten Gneisen nicht zu beobachten war.

Bei den folgenden Proben handelt es sich um Diatexite, die im Gelände als Granitoide vorliegen. Diese werden im Folgenden noch weiter unterteilt. Die Proben A60-06 A61-06, A74-06, A79-06 und A80-06 plotten nach Herron (1988) als Wacken und A76-06 im Feld der Tonsteine. Die Gesteine können in eine Gruppe mit granitischer (A61-06 und A80-06) und eine mit tonalitischer Zusammensetzung (A60-06, A74-06, A76-06 und A79-06) gruppiert werden. Die Mineralzusammensetzung der granitischen Proben ist Quarz, Plagioklas, Kalifeldspat, Biotit, Granat \pm Cordierit. Akzessorisch treten Zirkon und Apatit auf. Beide Gesteine sind mittelkörnig und rötlich. Probe A61-06 wurde im Südwesten der Sierras de Córdoba genommen und A80-06 in einem isolierten Bereich des San Carlos Massivs. Die tonalitischen Proben A60-06, A74-06, A76-06 und A79-06 sind ebenfalls mittelkörnig, allerdings gräulich. Die Hauptgemengteile sind Plagioklas, Quarz, Biotit und Granat \pm Kalifeldspat. Akzessorisch treten Zirkon, Apatit und Monazit auf. Hauptunterschied der beiden Gruppen sind das Auftreten bzw. Fehlen von Kalifeldspat sowie die Farbe der Gesteine. Die als Tonstein plottende tonalitische Probe A76-06 fällt durch einen höheren 
Biotitgehalt auf. Korngröße und und Farbe dieses Gesteins sind mit den anderen tonalitischen Diatexiten vergleichbar.

\subsection{Sierra Norte}

Von den zehn in der Sierra Norte genommenen Proben plotten zwei (A103-06 und A106-06) als Tonsteine. Die Probe A103-06 wurde in unmittelbarer Nähe zu einem Granit genommen. Aus diesem Grund wurde diese Probe kontaktmetamorph überprägt und liegt jetzt als Hornfels vor. Die andere als Tonstein bezeichnete Probe (A106-06) ist ein feinkörniges, niedriggradig metamorphes Gestein mit einem hohen Anteil an Phyllosilikaten und einigen feinkörnigen Quarzlagen. Da die Quarzlagen dynamisch rekristallisiert sein können, müssen die Temperaturen oberhalb von $300{ }^{\circ} \mathrm{C}$ gelegen haben. Im Gelände wurde diese Probe als Phyllit angesprochen. Vier Proben (A101-06, A105-06, A107-06 und A108-06) liegen im Diagram nach Herron (1988) direkt im Feld der Wacken. Im Verhältnis zu den feinkörnigen Proben A107-06 und A108-06 sind die Proben A101-06 und A105-06 grobkörniger, siltiger. In ihnen kommen größere Quarz- und Plagioklasklasten vor, die hauptsächlich von feinkörnigem Chlorit und Biotit umgeben werden. Die anderen beiden Proben wurden im Gelände als niedriggradige Metapelite angesprochen. Es handelt sich dabei um feinkörnige, homogene Gesteine aus Quarz und Glimmer ohne irgendeine Wechsellagerung. Drei Proben der Sierra Norte (A81-06, A100-06 und A104-06) liegen im Feld der Litharenite oder zumindest direkt daneben im Feld der Wacken. Makroskopisch wurden alle drei als Metapsammite beschrieben. Diese wurden im Fall von A81-06 und A100-06 durch eine Metamorphose mit Temperaturen oberhalb der Biotitisograde geprägt und im Fall von A10406 von einer Metamorphose oberhalb der Chloritisograde. Die Plagioklaskörner sind fast vollständig serizitisiert. Allerdings kann in der Probe A81-06 im Gegensatz zu den anderen Proben in einigen nicht vollständig serizitisierten Plagioklasen mikroskopisch eine magmatische Zonierung erkannt werden. Dies könnte auf einen vulkanischen Ursprung dieser Körner schließen lassen. Die Probe A102-06 liegt im Herron (1988) Diagram weit außerhalb aller anderen Proben. Die Lokation dieses Gesteins befindet sich in unmittelbarer Nähe zu der als Hornfels beschriebenen Probe A103-06 und sieht im Handstück ähnlich aus. Im Dünnschliff sind weitgehend isolierte Körner aus Plagioklas, polykristallinem Quarz und möglicherweise Chert in einer Matrix aus Biotit zu erkennen. Diese Probe ist im Herron (1988) Diagramm ebenfalls ein Tonstein, allerdings ein Fe-Tonstein. Da das Gestein deutlich oberhalb der direkt benachbarten Probe A103-06 liegt, besteht die Möglichkeit, dass A102-06 
durch Fluide, die in Bezug zu dem Granit stehen, metasomatisch überprägt wurde und aus diesem Grund deutlich außerhalb der Zusammensetzungen der anderen Gesteine zu finden ist. 


\section{Methodik}

Für die Geochemie und Isotopengeochemie wurden Gesamtgesteinsproben verwendet. Das Probenmaterial wurde mit Standardtechniken wie einem Backenbrecher grob zerkleinert und anschließend mit einer Achatkugelmühle analysefein gemahlen.

\subsection{Hauptelemente}

Die Hauptelemente wurden am Geoforschungszentrum GFZ in Potsdam mittels eines Panalytical XRF-PW 2400 gemessen.

\subsection{Aufschlüsse der Spurenelemente}

Die Aufschlüsse für die Spurenelemente wurden parallel in der Abteilung Isotopengeologie und der Abteilung Geochemie des Geowissenschaftlichen Zentrums der Georg-AugustUniversität Göttingen (GZG) vorgenommen. In der Abteilung Geochemie wurden die Proben in Autoklave wie bei Heinrich und Herrmann (1990) beschrieben aufgeschlossen. Es wurden ca. $100 \mathrm{mg}$ des Probepulvers mit $1 \mathrm{ml} \mathrm{HF}(40 \%)$ und $2 \mathrm{ml} \mathrm{HNO} 3(65 \%)$ bei $180{ }^{\circ} \mathrm{C}$ für mindestens $12 \mathrm{~h}$ gekocht und danach abgeraucht. Anschließend wurden die Proben mit $1 \mathrm{ml}$ $\mathrm{HF}(40 \%)$ und $1 \mathrm{ml} \mathrm{HClO}_{4}(70 \%)$ aufgenommen, bei $180{ }^{\circ} \mathrm{C}$ für $12 \mathrm{~h}$ gekocht und abgeraucht. Abschließend wurden die aufgeschlossenen Proben mit $2 \mathrm{ml} \mathrm{HNO}_{3}$ (65\%) aufgenommen und mit $\mathrm{H}_{2} \mathrm{O}$ zu $100 \mathrm{ml}$ verdünnt.

In der Abteilung Isotopengeologie wurde eine Zeit-Druck-Aufschluss Apparatur

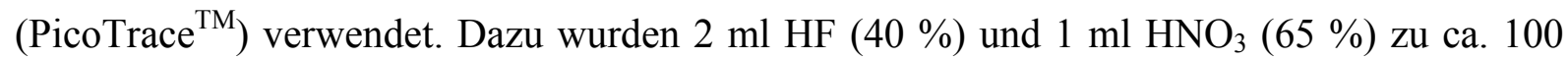
mg Probenpulver gegeben und nach einer Vorreaktion von $3 \mathrm{~h}$ abgeraucht. Anschließend

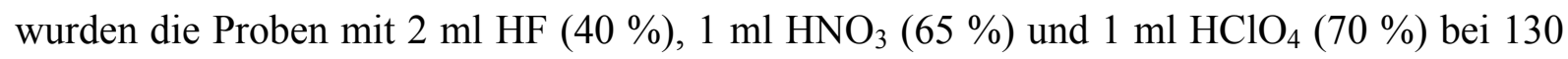
${ }^{\circ} \mathrm{C}$ für mindestens $72 \mathrm{~h}$ gekocht und darauf bei $140{ }^{\circ} \mathrm{C}$ abgeraucht. Danach wurden die Proben mit $5 \mathrm{ml} 6 \mathrm{~N} \mathrm{HCl}$ aufgenommen und für mindestens $48 \mathrm{~h}$ bei $160{ }^{\circ} \mathrm{C}$ gekocht. Um Nitrate zu erhalten, wurden nach dem Abrauchen der $\mathrm{HCl} 200 \mu \mathrm{HNO}_{3}(65 \%)$ dazugegeben und sofort wieder abgeraucht. Abschließend wurden die Proben mit $2 \mathrm{ml} \mathrm{HNO}_{3}(65 \%)$ aufgenommen und mit $\mathrm{H}_{2} \mathrm{O}$ auf $100 \mathrm{ml}$ verdünnt. In beiden Abteilungen wurde der gleiche Standard verwendet. Bei den Analysen konnte kein Unterschied der sich leicht unterscheidenden Aufschlussverfahren erkannt werden. Dies wurde mit Hilfe der Standards JA-2, MA-N und einigen Proben, die in beiden Abteilungen aufgeschlossen wurden, kontrolliert. Die ICP-MS 
Messungen wurden in der Abteilung Geochemie der Georg-August-Universität mit einer Plasma Quad II+ von VGTM durchgeführt. Die analytische Genauigkeit wurde dabei mit den Standards JA-2, QC-1 und MA-N überwacht und ergab einen Fehler zwischen 10-15\%.

\subsection{Nd-Isotopie}

Die Isotopenalysen für $\mathrm{Nd}$ und $\mathrm{Sm}$ wurden an repräsentativen Proben mittels konventinoneller Isotopenverdünnungstechniken durchgeführt. Die Proben wurden in Teflonbecher eingewogen und mit einer individuell berechneten Menge eines ${ }^{150}{ }^{\mathrm{Nd}-}{ }^{149} \mathrm{Sm}$

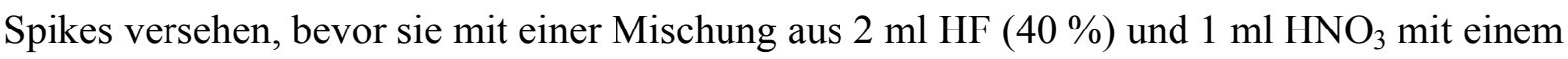
PicoTrace $^{\mathrm{TM}}$ Aufschlusssystem gekocht wurden. Die fertigen Aufschlüsse wurden mit Standard Kationaustausch-Techniken zur Anreicherung der Sm und Nd Fraktionen behandelt. Zur Ermittlung der Isotopenverhältnisse wurden SM und $\mathrm{Nd}$ zusammen mit $2.5 \mathrm{~N} \mathrm{HCl}$ auf vorbehandelte Re Doppelfilamente geladen. Die Messungen der Isotopenverhältnisse wurden an einem thermal ionisation mass spectrometer (TIMS) Finnigan Triton, in statischem Betrieb in der Abteilung Isotopengeologie der Georg-August-Universität (GZG) vorgenommen. Wiederholte Messungen eines internen Nd Standards ergaben ${ }^{143} \mathrm{Nd} /{ }^{144} \mathrm{Nd}$ Verhältnisse von $0.511798 \pm 0.000077(\mathrm{n}=71,2 \sigma)$ über den Zeitraum der Messungen für diese Arbeit. Die erhaltenen $\mathrm{Nd}$ Isotopenverhältnisse der Proben wurden auf einen ${ }^{146} \mathrm{Nd} /{ }^{144} \mathrm{Nd}$ Wert von 0.7219 normiert. Die Blindwerte waren durchweg unter $150 \mathrm{pg}$ für $\mathrm{Sm}$ und $\mathrm{Nd}$. Alle ${ }^{143} \mathrm{Nd} /{ }^{144} \mathrm{Nd}$ Verhältnisse werden mit ihrem internen $2 \sigma$ Fehler und den aus der Spikeberechnung resultierenden Ungenauigkeiten angegeben. Die Daten wurden nach dem Modell von Goldstein et al. (1984) berechnet.

\subsection{Pb-Isotopie}

Das Probenpulver wurde mit $\mathrm{HBr}$ vorbehandelt und mit einem $\mathrm{HF}-\mathrm{HNO}_{3}$ Aufschluss unter Zugabe von $\mathrm{HBO}_{3}$ nach Connelly et al. (2006) aufgeschlossen, danach abgeraucht und erneut mit $\mathrm{HNO}_{3}$ aufgenommen. Die Pb-Isotopenverhältnisse wurden am Institut für Geographie und Geologie der Universität Kopenhagen mit einem VG Sector 54 IT Massenspektrometer vorgenommen. Die chemische Separation des $\mathrm{Pb}$ wurde mit konventionellen Anionenaustauschsäulen mit $\mathrm{HBr}-\mathrm{HCl}$ ausgeführt, gefolgt von $200 \mathrm{ml}$ Teflon Reinigungskolonnen. Die Fraktionierung des $\mathrm{Pb}$ während der massenspektrometrischen Analysen, die im statischen Betrieb verliefen, wurde durch wiederhohlte Messungen des NBS 
981 Standards (Todt et al. 1993) kontrolliert. Die Fraktionierung beträgt $0.105 \pm 0.008 \%$ pro atomarer Masseneinheit ( $\mathrm{amu}, \mathrm{n}=12,2 \sigma$ ). Die Blindwerte blieben durchweg unter $50 \mathrm{pg}$, womit sichergestellt ist, dass die Pb-Isotopie nicht signifikant gestört wird.

\subsection{U-Pb SHRIMP Zirkondatierungen}

Die Zirkonpräparate der Proben A73-05, A93-05, A7-06 und A8-06 wurden mittels SHRIMP II am Centre of Isotopic Research, VSEGEI, St. Petersburg, Russia gemessen. Dazu wurden die Körner zusammen mit Stücken des TEMORA (Middledale Gabbroic Diorite, New South Wales, Australia) und 91500 (Geostandard Zirkon) Referenzzirkonen in Epoxidharz gegossen. Die Körner wurden etwa in der Mitte zerteilt und poliert. Von jedem Zirkon liegen Kathodolumineszenzbilder vor. Diese wurden verwendet, um mittels der Internstruktur der Körner bestimmte Punkte festzulegen, die analysiert wurden. Jede Analyse besteht aus 5 Durchläufen über den gesamten Massebereich. Jeder Analysepunkt hat einen Durchmesser von etwa $25 \mu \mathrm{m}$ und die Intensität des primären Strahls betrug $2 \mathrm{nA}$. Die Daten wurden ähnlich wie bei Williams (1998 und darin enthaltener Referenzen) behandelt. Dabei wurde das SQUID Excel Macro von Ludwig (2000) verwendet. Die Pb/U Verhältnisse wurden relativ $\mathrm{zu}$ einem Wert von 0,0662 für das ${ }^{206} \mathrm{~Pb} /{ }^{238} \mathrm{U}$ Verhältnis der TEMORA Referenzzirkone, die ein Alter von 416,75 Ma besitzen (Black und Kamo 2003) normiert. Fehler der einzelnen Analysen (Verhältnisse und Alter) werden mit $1 \sigma$ angegeben.

Die Proben A30-01, A1-08, A3-08 und A4-08 wurden an der Research School of Earth Sciences (RSES, Canberra) zusammen mit RSES Referenzzirkonen FC1 in Epoxidharz gegossen. Von allen Zirkonen wurden Kathodlumineszenzbilder angefertigt und verwendet, um die Analysepunkte an den einzelnen Zirkonkörnern festzulegen. Der Durchmesser der Analysepunkte beträgt ca. $25 \mu \mathrm{m}$. Dabei wurde ein primärer $\mathrm{O}_{2}$ Ionenstrahl von $4-6 \mathrm{nA}$ verwendet. Für die Kalibrierung der Zirkone wurden die $\mathrm{Pb} / \mathrm{U}$ Werte relativ zu einem Wert von $0,1859 \mathrm{der}{ }^{206} \mathrm{~Pb} /{ }^{238} \mathrm{U}$ Verhältnisse der FC1 Referenzzirkone, die ein Alter von $1099 \mathrm{Ma}$ aufweisen (Paces und Miller) normiert. U- und Th-Konzentrationen wurden relativ zu dem Standard SL13 ermittelt. Der Fehler Standardkalibrierung beträgt bei der SHRIMP II 0,25 \%. Fehler der einzelnen Analysen (Verhältnisse und Alter) werden mit $1 \sigma$ angegeben. Durchschnittsalter oder konkordante Alter (Ludwig 2000) werden mit $2 \sigma$, wenn nicht anders beschrieben, angegeben. Diese enthalten, wenn nötig, die Fehler der Standardkalibrierung. Die angegebenen Concordia Diagramme und berechneten Alter wurden mit Isoplot/Ex 3.0 (Ludwig 2003) durchgeführt. 


\section{Vorstellung der Ergebnisse}

\subsection{Chemical Index of Alteration}

Liefergebietsanalysen mithilfe von Hauptelementen an (Meta)Sedimenten sind nur dann sinnvoll, wenn diese weder bei der Diagenese, der Metamorphose noch bei der Verwitterung beeinflusst wurden (McLennan et al. 1993). Die Hauptelemente bzw. bestimmte Verhältnisse dieser Elemente können Hinweise bezüglich der Liefergebiete des sie aufbauenden klastischen, erodierten Materials geben (z.B. Boles und Franks 1979; Nesbit et al. 1996; Zimmermann und Bahlburg 2003). Um den Einfluss der chemischen Verwitterung und einer evtl. auftretenden Metasomatose zu bestimmen, kann der Chemical Index of Alteration (CIA) verwendet werden (Nesbit und Young 1982). Dieser Index wird in Mol \% als $\mathrm{Al}_{2} \mathrm{O}_{3} /\left(\mathrm{Al}_{2} \mathrm{O}_{3}+\right.$ $\left.\mathrm{CaO}^{*}+\mathrm{Na}_{2} \mathrm{O}+\mathrm{K}_{2} \mathrm{O}\right) \times 100$ berechnet. $\mathrm{CaO}^{*}$ steht dabei für Kalzium, das ausschließlich aus Silikaten stammt. Der Kalziumgehalt aus möglicherweise auftretenden Karbonaten und Apatit muss subtrahiert werden. Der korrigierte Wert für $\mathrm{CaO}$ wird erhalten, indem von dem molaren Anteil an $\mathrm{CaO}$ die Mole $\mathrm{CO}_{2}$ (Karbonat) und 10/3 der Mole $\mathrm{P}_{2} \mathrm{O}_{5}$ (Apatit) subtrahiert werden. Der CIA bildet hauptsächlich die Verwitterung von Feldspäten ab. Aufgrund unterschiedlicher Zusammensetzungen des Ausgangsmaterials liegen die Startpunkte der Gesteine nicht am gleichen Punkt im CIA Diagramm (Abb. 5). Frische granitische Proben haben einen CIA Wert zwischen 45 und 55. Tonsteine haben üblicherweise Werte zwischen 70 und 75. Chlorite und Kaolinit haben Werte von 100. Zusätzlich zu den CIA Werten bestimmt bei Granitoiden das Verhältnis von Kalifeldspat und Plagioklas die Lage im Diagramm. Da Tonalite fast keinen Kalifeldspat enthalten, liegen sie bei einem CIA Wert zwischen 45 und 55 auf der linken Seite des Diagramms. Granite hingegen liegen bei gleichen CIA Werten weiter rechts, da ihr $\mathrm{K}_{2} \mathrm{O} /\left(\mathrm{CaO}^{*}+\mathrm{Na}_{2} \mathrm{O}\right)$ Verhältnis aufgrund eines höheren Kalifeldspat- und geringeren Plagioklasgehalts höher ist. Ein idealer Verlauf des Verwitterungstrends würde parallel zur A-CN-Achse $\left(\mathrm{Al}_{2} \mathrm{O}_{3}-\mathrm{CaO} * \mathrm{Na}_{2} \mathrm{O}\right.$-Achse $)$ verlaufen (Abb. 5).

Von den 66 genommenen Proben wurden 65 im CIA Diagramm dargestellt. Die Probe A10206 wurde aufgrund ihres wahrscheinlich gestörten Verhältnisses der Hauptelemente nicht berücksichtigt.

Die Proben aus dem Nogolí Metamorphic Complex zeigen eine Entwicklung, die vom idealen Verwitterungstrend abweicht. Den höchsten CIA Wert weist die Probe A39-01 auf. Dies ist erwartet worden, weil es sich hierbei nach der Klassifikation von Herron (1988) um einen 


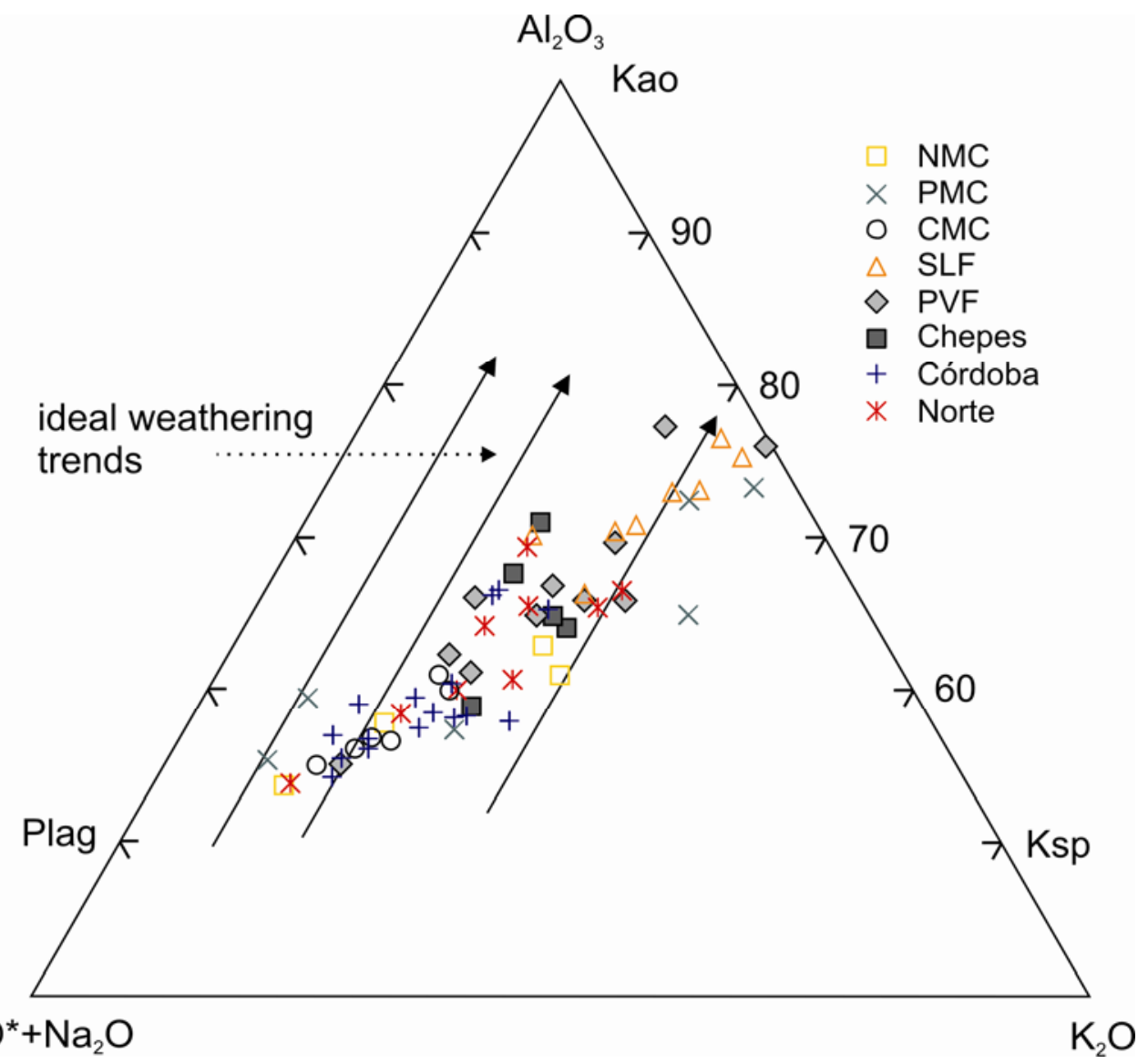

Abbildung 5: Chemical Index of Alteration (CIA), berechnet nach Nesbit und Young (1982) für die Proben der östlichen Sierras Pampeanas und der Puncoviscana Formation der Cordillera Oriental. Die Pfeile zeichnen einen idealen Verwitterungstrend eines Granits (rechts), eines Granodiorits (Mitte) und eines Tonalits (links) nach. Ein lediglich leicht vom idealen Verwitterungstrend abweichender Verlauf der Datenpunkte kann beobachtet werden. Probe A102-06 kann aufgrund eines extrem niedrigen CIA von 36 nicht dargestellt werden.

Tonstein handelt. Die anderen drei Proben haben alle ähnliche $\mathrm{SiO}_{2}$ Gehalte zwischen 75 und $79 \%$ und auch die $\mathrm{Al}_{2} \mathrm{O}_{3}$ Konzentrationen zwischen 10,0 und 11,6\% weichen nicht stark voneinander ab. Trotzdem liegt die Probe A16-06 im Bereich von A39-01. Ursächlich hierfür ist der erhöhte $\mathrm{K}_{2} \mathrm{O}$-Gehalt dieses Gesteins, der bei 3,4 \% liegt und nicht zwischen 1,5 und 1,8\%, so wie bei A7-06 und A8-06. Bereits makroskopisch fällt die große Menge Muskovit auf, welche sekundär die Foliation überwachsen. Dies könnte für eine Kaliummetasomatose bei der Probe A16-06 sprechen.

Im Pringles Metamorphic Complex liegen die Proben A13-06 und A19-06 auf der linken Seite des Diagramms (Abb. 5) und scheinen einen idealen Verwitterungstrend abzubilden. Sie weisen mit 85 und $88 \%$ den höchsten $\mathrm{SiO}_{2}$ Gehalt auf. Im rechten Bereich des Diagramms sind drei Proben (A14-06, A15-06 und A18-06) abgebildet, die entweder einen idealen Trend für Granite abbilden, oder einer starken Metasomatose ausgesetzt waren. Die Probe A15-06 wurde im Gelände als metapelitisch angesprochen, Probe A18-06 als psammopelitisch. Deshalb ist davon auszugehen, dass bereits bei der Sedimentation ein erhöhter Kaliumgehalt 
vorlag, der durch einen hohen Anteil an Biotit widergespiegelt wird und nicht das Resultat einer Metasomatose ist. Bei A14-06 wird dagegen eine Kaliummetasomatose angenommen, da sehr viel Muskovit die Foliation überwächst. Bei der Probe A5-01 sind solche Phänomene nicht zu beobachten und es wird davon ausgegangen, dass sie mehr oder weniger ungestört in ihrer Geochemie vorliegt.

Vier Proben (A61-05, A73-05, A9-06 und A10-06) des Conlara Metamorphic Complex (Abb. 5) liegen im linken unteren Bereich des Diagramms. Sie weisen einen leichten Trend zu erhöhten Kaliumwerten oder aber einen leichten Unterschied des Ausgangsmaterials auf. Zwei weitere Proben (A21-01 und A23-01) haben dem beschriebenen Trend folgend etwas höhere CIA Werte. Die Zusammensetzung der Proben des Conlara Metamorphic Complex ist fast identisch, bei den Proben mit höherem CIA Wert fällt jedoch eine leicht erhöhte $\mathrm{K}_{2} \mathrm{O}$ Konzentration (2,6 und 2,8 mmol verglichen mit 1,7-2,5 mmol bei den anderen Proben) und geringere $\mathrm{CaO}^{*}$ Werte (14 und $23 \mathrm{mmol}$ im Vergleich $\mathrm{zu} 26-34 \mathrm{mmol}$ ) auf. Auch im Conlara Metamorphic Complex kann es vorkommen, dass Muskovit die Foliation überwächst, jedoch in einem geringeren Maße als dies im Nogolí Metamorphic Complex oder dem Pringles Metamorphic Complex der Fall ist.

Die aus der San Luis Formation genommenen Proben liegen im rechten oberen Bereich des Diagramms (Abb. 5) eng zusammen. Lediglich die litharenitische Probe A9-01 liegt außerhalb dieses Bereichs. Dies könnte auf eine unterschiedliche Ursprungszusammensetzung dieser Probe im Vergleich $\mathrm{zu}$ den anderen sprechen oder für eine unterschiedliche Verwitterung bzw. Metasomatose. Bei den übrigen sieben Proben ist eine leichte Abweichung vom idealen Verwitterungstrend auszumachen. Der CIA liegt bei den Proben der San Luis Formation, mit Ausnahme der Arkose A12-06, zwischen 70 und 76. Diese hohen Werte haben ihren Ursprung in dem hohen Anteil an Phyllosilikaten, z.B. Chlorit. Bei A12-06 treten aufgrund des hohen $\mathrm{SiO}_{2}$-Anteils (82\%) weniger Phyllosilikate auf. Deshalb ist der CIA hier mit 66 geringer.

Auch in der Puncoviscana Formation ist der CIA bei den beiden als Tonstein (Herron 1988) klassifizierten Proben A85-06 und A86-06 mit 76 und 77 aus den gleichen Gründen am höchsten. In dieser Einheit ist eine klar abweichende Tendenz vom idealen Verwitterungstrend zu beobachten, wenn bei den unterschiedlichen Proben jeweils ähnliche Ausgangsmaterialien vorlagen. Auffällig ist Probe A96-06, die mit 55 einen deutlich geringeren CIA aufweist, als alle anderen Proben aus diesem Gebiet. Hierbei handelt es sich um einen gebänderten Gneis und nicht um ein niedriggradig metamorphes Gestein. Der 
zweite gebänderte Gneis (A97-06) hat mit 61 ebenfalls einen geringen CIA, allerdings zeigt diese Probe ein deutlich erhöhtes $\mathrm{K}_{2} \mathrm{O} / \mathrm{Na}_{2} \mathrm{O}+\mathrm{CaO} *$ Verhältnis an.

In den Sierras de Chepes liegen die Proben zwischen dem Verwitterungstrend von granitischem und granodioritischem Ausgangsmaterial. Die Gesteine zeigen keine Anzeichen eines Abweichens vom idealen Verwitterungstrend, obwohl der CIA zwischen 59 und 71, also relativ hoch liegt. Die Proben scheinen weder durch die Verwitterung noch durch eine Metasomatose beeinflusst worden zu sein.

Bei den Proben der Sierras de Córdoba fallen sechs Datenpunkte im linken unteren Bereich des Diagramms auf. Bei diesen Punkten handelt es sich um die Proben A62-06, A65-06, A6606, A70-06, A75-06 und A77-06. Bei diesen Gesteinen handelt es sich um mittelgradig metamorphe Gneise und bei A77-06 um ein hochgradig metamorphes stromatitisches Gestein. Diatexite kommen in dieser Gruppe nicht vor. Die übrigen drei niedrig- bis mittelgradig metamorphen Gesteine A63-06, A64-06 und A68-06 sind zwar leicht zu höheren Kaliumverhältnissen verschoben, liegen aber auf einer Linie, dem idealen Verwitterungsprozess folgend. Von den vier tonalitischen Proben A60-06, A74-06, A76-06 und A79-06 liegen alle bis auf A79-06 auf einem idealen Verwitterungstrend. Die Probe A7906 ist aufgrund ihrer hohen $\mathrm{K}_{2} \mathrm{O}$ Konzentration, die in einem hohen Biotit Anteil vermutet wird, nach rechts verschoben. Der Grund, warum diese Probe von den drei anderen mit tonalitischer Zusammensetzung abweicht, muss also nicht zwangsläufig in einer Metasomatose oder unterschiedlichen Verwitterung zu suchen sein, sondern könnte auch auf eine unterschiedliche Ursprungszusammensetzung hindeuten.

Eine weitere $\mathrm{zu}$ einem höheren $\mathrm{K}_{2} \mathrm{O} /\left(\mathrm{Na}_{2} \mathrm{O}+\mathrm{CaO} *\right)$ verschobene Probe ist A78-06. Ob bei dieser stromatitischen Probe ebenfalls der hohe Biotitanteil oder Fluide ursächlich für die leicht von den anderen Datenpunkten abweichende Position im Diagramm ist, kann nicht eindeutig geklärt werden. Die beiden Diatexite mit granitischer Zusammensetzung A61-06 und A80-06 liegen im Diagramm sehr eng zusammen, sodass eine ähnliche Verwitterung angenommen wird.

In der Sierra Norte wurde die Probe A102-06 nicht mit in die Diskussion über den CIA einbezogen, da diese einen unnatürlich niedrigen CIA von 36 aufweist und außerdem abweichende Hauptelementverteilungen hat (Anhang 1). Es muss sich um einen vulkanischen Eintrag in dieses Metasediment handeln, der nicht charakteristisch für das Liefergebiet ist. Eine weitere Möglichkeit ist, dass ein metasomatischer Prozesse das Gestein beeinflusst hat. Die Probe A104-06 hat den geringsten CIA und das größte CN/K Verhältnis. Diese Probe ist zusammen mit A81-06 und A100-06 mit ca. $75 \%$ am $\mathrm{SiO}_{2}$ reichsten, allerdings haben die 
anderen beiden Gesteine ein deutlich geringeres $\mathrm{CN} / \mathrm{K}$ Verhältnis, welches vom idealen Verwitterungstrend abweicht. Der makro-, bzw. mikroskopische Unterschied zwischen diesen Proben ist der Metamorphosegrad, der bei A104-06 oberhalb der Chloritisograde liegt und bei den anderen beiden Proben oberhalb der Biotitisograde. Die höhere Metamorphose könnte also in diesem Fall eine Abweichung vom idealen Trend bewirkt haben. Die beiden bei Herron (1988) als Tonstein plottenden Gesteine (A103-06 und A106-06) liegen aufgrund ihrer hohen $\mathrm{K}_{2} \mathrm{O}$ Konzentration etwas außerhalb der restlichen Datenpunkte der Sierra Norte (Abb. 5). Die übrigen vier Proben (Wacken nach Herron 1988) liegen auf einem idealen Verwitterungstrend, sodass die Verwitterung bei den Proben der Sierra Norte keine deutlich beeinflussenden Auswirkungen haben sollte.

Bei den Daten der Sierras Pampeanas scheint auf den ersten Blick auf die Datenpunkte eine klare Abweichung vom idealen Verwitterungstrend vorzuliegen. Bei differenzierter Betrachtung der unterschiedlichen Gesteine fällt jedoch auf, dass sich dies bis auf ein oder zwei Ausnahmen nicht bestätigt und keine generelle Tendenz abweichend vom idealen Verwitterungstrend vorliegt.

\subsection{Gesamtgesteins-Geochemie}

\subsubsection{Hauptelemente}

Um mit Hilfe von Hauptelementen Hinweise auf die Provenienz (meta)sedimentärer Gesteine zu erlangen, kann das Diagramm (Abb. 6) von Bhatia (1983) verwendet werden. In diesem Diagramm wird der prozentuale $\mathrm{TiO}_{2}$ Gehalt gegen die Summe von $\mathrm{Fe}_{2} \mathrm{O}_{3}+\mathrm{MgO}$ aufgetragen. Je nach Lage in diesem Diagramm kann dies Hinweise auf unterschiedliche geotektonische Milieus wie den ozeanischen Inselbogen (A), kontinentalen Inselbogen (B), aktiven Kontinentalrand (C) oder passiven Kontinentalrand (D) liefern. Da Bhatia (1983) dieses Diagramm für Sandsteine definiert hat, werden die Proben, die als Tonstein klassifiziert wurden, nicht verwendet. Bei den verwendeten Proben fällt ein linearer Anstieg der $\mathrm{TiO}_{2}$ Werte bei ebenfalls steigenden $\mathrm{Fe}_{2} \mathrm{O}_{3}+\mathrm{MgO}$ Gehalten auf (Abb. 6). Dieser Trend steht in Verbindung zur $\mathrm{SiO}_{2}$ Konzentration der Proben. Je höher der $\mathrm{SiO}_{2}$-Gehalt desto niedriger die Werte für $\mathrm{TiO}_{2}$ und $\mathrm{Fe}_{2} \mathrm{O}_{3}+\mathrm{MgO}$. Dieser Trend wird allerdings von einigen Proben nicht eingehalten. Die Proben des Nogolí Metamorphic Complex liegen alle im Feld für aktiven Kontinentalrand und kontinentalen Inselbogen. Beim Pringles Metamorphic Complex liegen zwei Proben im Feld für passiven Kontinentalrand, ein Feld, in dem keine 


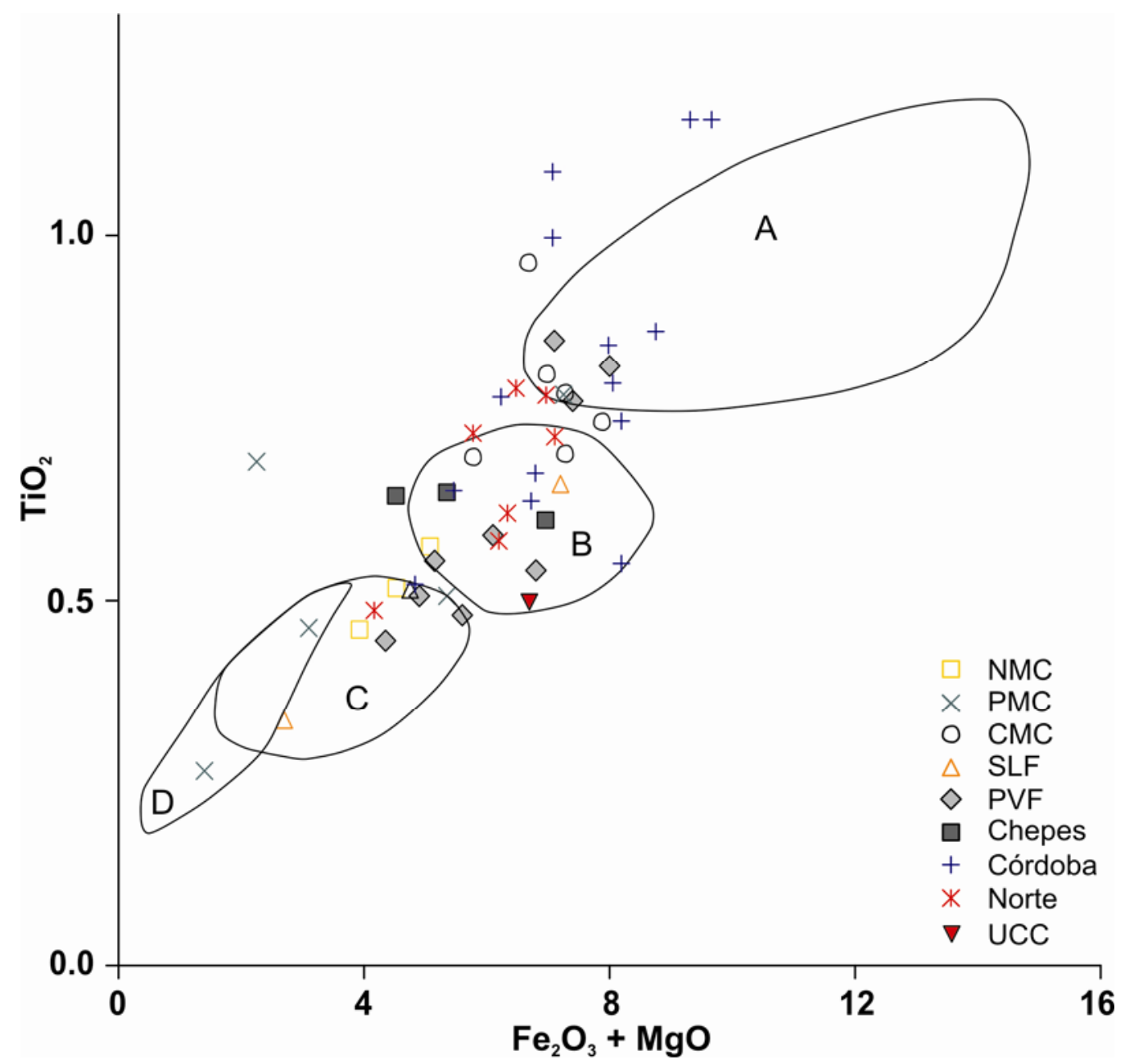

Abbildung 6: Anhand des vorliegenden $\mathrm{TiO}_{2}$ vs. $\mathrm{Fe}_{2} \mathrm{O}_{3} \mathrm{t}+\mathrm{MgO}$ Diagramms (Bhatia 1983) soll versucht werden, eine bestimmte geotektonische Position für die Proben der östlichen Sierras Pampeanas und der Puncoviscana Formation aus der Cordillera Oriental zu ermitteln. A: ozeanischer Inselbogen, B: kontinentaler Inselbogen, C: aktiver Kontinentalrand, D: passiver Kontinentalrand.

Probe einer anderen Einheit liegt. Eine Probe liegt zwischen kontinentalem Inselbogen und ozeanischem Inselbogen und eine Probe hat einen $\mathrm{Fe}_{2} \mathrm{O}_{3}+\mathrm{MgO}$ Gehalt eines passiven Kontinentalrandes, jedoch eine deutlich erhöhte $\mathrm{TiO}_{2}$ Konzentration, so dass diese Probe weit außerhalb der angegebenen Felder liegt. Der relativ hohe $\mathrm{TiO}_{2}$ Wert und die in dieser Probe ebenfalls sehr hohe Zirkoniumkonzentration von 760 ppm könnten auf einen generell erhöhten Schwermineralgehalt in Form einer Seife hindeuten, die allerdings weder mikronoch makroskopisch entdeckt wurde, sodass diese Probe in Bezug auf das geotektonische Milieu eher zu geringeren $\mathrm{TiO}_{2}$ Werten, also in Richtung eines passiven Kontinentalrandes korrigiert werden müsste als zu höheren $\mathrm{Fe}_{2} \mathrm{O}_{3}+\mathrm{MgO}$ Werten in Richtung kontinentaler Inselbogen. Die Proben des Conlara Metamorphic Complex liegen entweder im Feld für kontinentalen oder ozeanischen Inselbogen. Die Probe A10-06 weist erhöhte $\mathrm{TiO}_{2}$ Werte auf. Der Grund hierfür könnte an einer erhöhten Anzahl von im Dünnschliff zu beobachtenden 
Ilmenitkörnern liegen. Im Gegensatz dazu liegen die Proben der San Luis Formation wie der Großteil der Proben aus der Sierra de San Luis in den Feldern kontinentaler Inselbogen und aktiver Kontinentalrand. Bei den Gesteinen der Puncoviscana Formation zeigt sich ein größeres Spektrum, das vom aktiven Kontinentalrand (A87-06, A90-06 und A94-06) über kontinentalen Inselbogen (A88-06, A91-06 und A93-06) bis hin zu ozeanischem Inselbogen reicht (A89-06, A96-06 und A97-06). Der $\mathrm{SiO}_{2}-$ Gehalt scheint in dieser Einheit nicht die Hauptursache für die Unterschiede zu sein, denn die Proben A90-06 und A94-06, die im Feld vom aktiven Kontinentalrand liegen, haben nur geringfügig höhere Konzentrationen (70.9 und $69.6 \%$ ) als die beiden gebänderten Gneise A96-06 und A97-06 (70.2 und 68.1\%), deren Datenpunkte im Feld für ozeanischen Inselbogen liegen. Die Gesteine aus der Sierras de Chepes liegen alle im Feld für kontinentalen Inselbogen und folgen einem linearen Trend zu höheren $\mathrm{TiO}_{2}-$ und $\mathrm{MgO}+\mathrm{Fe}_{2} \mathrm{O}_{3}$-Werten. Die Datenpunkte der Gesteine aus den Sierras de Córdoba sind stark verteilt. Während A79-06 im Feld für aktiven Kontinentalrand liegt, haben die Proben A60-06, A68-06, A70-06 und A80-06 so hohe $\mathrm{TiO}_{2}$ Werte, dass sie sogar oberhalb des Feldes für ozeanische Inselbögen liegen. Bei den Proben, die im Feld für kontinentaler Inselbogen oder aktiven Kontinentalrand liegen, handelt es sich entweder um mittelgradig metamorphe Gesteine (A62-06, A63-06 und A75-06) oder um hochgradig metamorphe Diatexite (A75-06 und A79-06) aus dem San Carlos Massiv. Bei den übrigen Datenpunkten ist eine Unterscheidung schwierig, da bestimmten Bereichen im Diagramm kaum noch einzelne Gesteinsgruppen wie Diatexite, mittelgradig oder hochgradig metamorphe Gesteine zugeordnet werden können. Einzige weitere Auffälligkeit ist, dass ein Diatexit mit tonalitischer Zusammensetzung (A60-06) und ein Diatexit mit granitischer Zusammensetzung (A80-06) die mit Abstand höchsten Werte sowohl für $\mathrm{TiO}_{2}$, als auch für $\mathrm{Fe}_{2} \mathrm{O}_{3}+\mathrm{MgO}$ haben. Die Datenpunkte der Gesteine der Sierra Norte liegen mit Ausnahme der litharenitischen Probe A104-06, die im Feld für aktiven Kontinentalrand liegt, alle im Feld für kontinentalen Inselbogen oder leicht darüber. Die anderen beiden Litharenite haben trotz vergleichbarer $\mathrm{SiO}_{2}$ Gehalte deutlich höhere Konzentrationen von $\mathrm{TiO}_{2}$ und $\mathrm{Fe}_{2} \mathrm{O}_{3}+\mathrm{MgO}$. Eine unterschiedliche Zusammensetzung dieser Proben konnte Bereits im CIA Diagramm beobachtet werden. Die verbleibenden vier Proben A101-06, A105-06, A107-06 und A108-06 unterscheiden sich in ihren Hauptelementen lediglich marginal, so auch in der Konzentration an $\mathrm{Fe}_{2} \mathrm{O}_{3}+\mathrm{MgO}$ zwischen $6.1-7.0 \%$. Der Unterschied in dem vorliegenden Diagramm bezieht sich auf die $\mathrm{TiO}_{2}$ Konzentration, die zwischen $0.58-0.78 \%$ liegt.

Problematisch bei der Liefergebietsanalyse mithilfe von Hauptelementen bleibt allerdings deren Verlässlichkeit. Sie sollten stets mit Vorsicht gehandhabt werden, da z.B. Armstrong- 
Altrin und Verma (2005) gezeigt haben, dass diese Elemente aufgrund einer möglichen Mobilisierung Probleme bei der korrekten Interpretation aufwerfen können.

\subsubsection{Spurenelemente}

Wegen ihrer geringen Mobilität und Löslichkeit während sedimentärer und diagenetischer Prozesse und ihrer geringen Verweilzeit im Meerwasser sind high field strength elements (HFSE) und einige Spurenelemente sehr gut geeignet, um einen Beitrag zur Liefergebietsanalyse und zur Entschlüsselung des geotektonischen Milieus zu liefern (Taylor und McLennan 1985; Bhatia und Crook 1986; Floyd und Leveridge 1987; McLennan 1989; McLennan et al. 1990).

Aufgrund gegensätzlicher Kompatibilitäten von Thorium und Scandium weichen die Elementverhältnisse dieser Elemente in basischen Gesteinen stark von den Verhältnissen in sauren Gesteinen ab. Thorium ist stark inkompatibel und wird deshalb in partiellen Schmelzen im oberen Mantel und der Erdkruste angereichert. Scandium dagegen ist ein kompatibles Element und wird nur zu geringen Konzentrationen in der partiellen Schmelze vorkommen. Erst bei stärkerer Aufschmelzung steigt die Scandium-Konzentration an. Dies ist bei basischen Gesteinen und Vulkaniten zu beobachten. Aus diesen Gründen ist das $\mathrm{Th} / \mathrm{Sc}$ Verhältnis sehr gut geeignet, um mafische Komponenten in klastischem Material nachzuweisen. Als Grenzwert für oberkrustale Gesteine hat McLennan (2001) das Th/Sc Verhältnis von 0.8 angegeben (Abb. 7). Die $\mathrm{SiO}_{2}$-reichen Proben aus dem Nogolí Metamorphic Complex haben Th/Sc Verhältnisse von 1.4 - 1.6, die als Tonstein klassifizierte Probe hat einen etwas niedrigeren Wert von 1.1. Der Tonstein aus dem Pringles Metamorphic Complex weist ebenfalls ein Verhältnis von 1.1 auf. Die Proben mit mittleren $\mathrm{SiO}_{2}$ Gehalten zeigen Werte von 1.4 - 2.1. Die Werte der $\mathrm{SiO}_{2}$ reichsten Proben (A13-06 mit 88 \% und A1906 mit $84 \%$ ) sind stark erhöht und ergeben 6.7 und 5.1. Dies sind die höchsten Werte der gesamten Kollektion. Der nächst niedere Wert im gesamten Arbeitsgebiet liegt bei 2.7 (A1206 und A80-06). Die Werte des Conlara Metamorphic Complex liegen zwischen 0.9 und 1.4. Lediglich die schwach metamorphe Probe A9-06 hat einen geringeren Wert von 0.7. In der San Luis Formation ergeben sich Th/Sc Verhältnisse von 0.9 - 1.7. In diesem Gebiet hat, wie im Pringles Metamorphic Complex auch, die $\mathrm{SiO}_{2}$-reichste Probe einen erhöhten Wert von 2.7. Die Quotienten aus Th und Sc liegen in der Puncoviscana Formation zwischen $0.7-1.3$. Die einzige Ausnahme bildet auch hier die $\mathrm{SiO}_{2}$ reichste Probe A87-06 (75 \%) mit 1.9. Die niedrigsten Werte mit 0.7 haben in dieser Einheit die beiden schwach metamorphen Tonsteine 


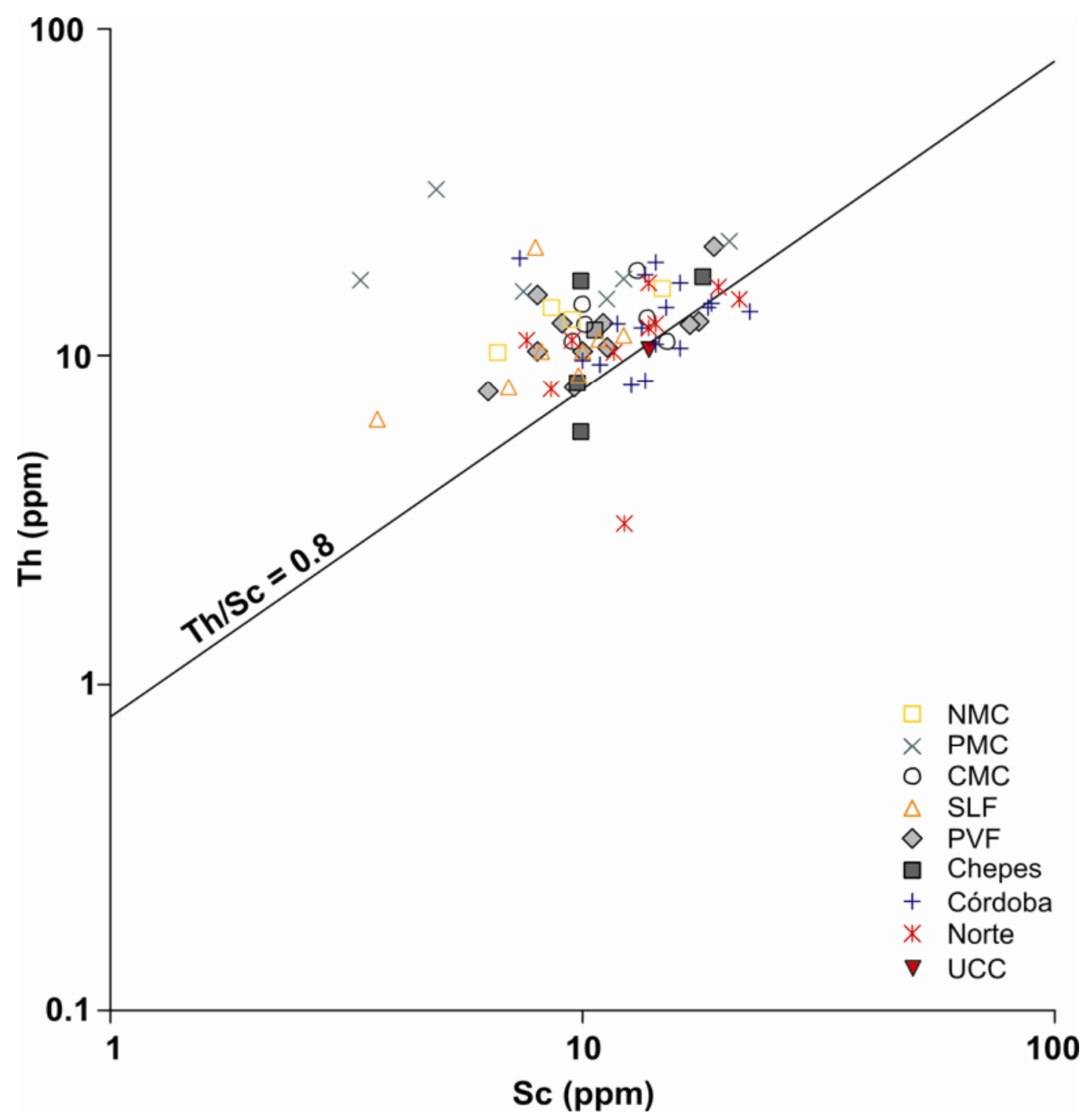

Abbildung 7: Th vs. Sc Diagramm zur Ermittlung des Liefergebiets und der Zusammensetzung der Gesteine der unterschiedlichen Einheiten der östlichen Sierras Pampeanas und der Puncoviscana Formation der Cordillera Oriental. Die Linie, die ein Th/Sc Verhältnis von 0.8 nachzeichnet, markiert den von McLennan et al. (1990) ermittelten unteren Grenzwert für Gesteine der oberen Erdkruste.

A85-06 und A86-06. Die als Phyllite und schwach metamorpher Sandstein angesprochenen Proben aus den Sierras de Chepes haben Werte von 0.6 - 0.9, die beiden gebänderten Gneise dagegen haben höhere Werte von 1.1 und 1.7. Die durchschnittlichen Th/Sc Verhältnisse sind in den Sierras de Córdoba am geringsten. Bis auf drei Proben liegen die Werte zwischen $0.6-$ 1.1. Dabei ist es unerheblich, um welche Art von Gestein es sich handelt. Die beiden niedrigbis mittelgradig metamorphen Gesteine A68-06 und A70-06 haben etwas höhere Werte von 1.4 und 1.3 und die diatexitische Probe A80-06 weist mit 2.7 den höchsten Wert in dieser Einheit auf. Fünf Proben der Sierra Norte (A101-06, A103-06, A106-06, A107-06 und A10806) haben Th/Sc Werte um 0.9. Weitere drei Proben (A100-06, A104-06 und A105-06) haben höhere Werte von 1.2 - 1.5. Die Probe A102-06 hat ein Verhältnis von 0.7. Dieses Gestein 
zeigte bereits bei den Hauptelementen und im Dünnschliff Auffälligkeiten, sodass dieser Wert möglicherweise durch sekundäre Prozesse zustande kam und nicht den ursprünglichen $\mathrm{Th} / \mathrm{Sc}$ Wert reflektiert. Der geringste Wert von 0.3 liegt bei A81-06 vor. Bei dieser Probe fielen im Dünnschliff im Gegensatz zu allen anderen Gesteinen zonierte Plagioklase auf. Dies könnte auf eine unterschiedliche, in diesem Fall vulkanische Provenienz schließen lassen.

Die oben genannten Verhältnisse von $\mathrm{Th} / \mathrm{Sc}$ sind ein Indikator für die Reife von Sedimenten. Das $\mathrm{Zr} / \mathrm{Sc}$ Verhältnis spiegelt die Anreicherung von Zirkon wieder. Da Zirkon ein äußerst stabiles Mineral in Bezug auf die Verwitterung ist, wird es z.B. beim Transport der Sedimente von der Erosion bis zur Ablagerung angereichert und das $\mathrm{Zr} / \mathrm{Sc}$ Verhältnis steigt (McLennan et al. 1990, 1993). In dem vorliegenden Diagramm (Abb. 8) ist der Recycling Trend der Proben bis auf den Pringles Metamorphic Complex gering ausgeprägt, es sind jedoch auch zwischen den anderen Einheiten Unterschiede sichtbar. Die Proben des Nogolí Metamorphic Complex haben alle $\mathrm{Th} / \mathrm{Sc}$ Werte über 1.0 und $\mathrm{Zr} / \mathrm{Sc}$ Werte zwischen 10 und 20. Einzige Ausnahme bildet A16-06 mit einem leicht erhöhten Wert von 32. Vier Zr/Sc Verhältnisse des Pringles Metamorphic Complex liegen im Bereich der anderen Einheiten zwischen 10 und 30, haben allerdings erhöhte Th/Sc Werte. Zwei Proben aus dieser Einheit fallen aus den Gesamtdaten heraus. Sie haben nicht nur Th/Sc Daten über 5, sondern auch deutlich erhöhte $\mathrm{Zr} / \mathrm{Sc}$ Verhältnisse von 55 und 220, was für eine deutliche Anreicherung von Zirkon spricht. Die Zr Konzentration von A13-06 liegt bei etwa 270 ppm, was nur eine leichte Erhöhung gegenüber den vier Proben mit geringeren $\mathrm{Zr} / \mathrm{Sc}$ Verhältnissen spricht. Allerdings ist der $\mathrm{SiO}_{2}$ Gehalt mit 88 \% sehr hoch und aufgrund dieses Verdünnungseffektes die Sc Konzentration mit 5 ppm gering (Durchschnitt dieser Einheit 10 ppm). Auch A19-06 weist einen hohen $\mathrm{SiO}_{2}$ Wert von $84 \%$ auf. Der hier extrem hohe $\mathrm{Zr} / \mathrm{Sc}$ Wert geht nicht nur auf eine geringe Sc Konzentration von 3 ppm zurück, sondern auch auf den mit 750 ppm mit Abstand höchsten Wert für Zr. Der hohe $\mathrm{SiO}_{2}$ Gehalt dieser Proben birgt die Gefahr, dass wenige Schwerminerale einen hohen Effekt auf die Konzentration bzw. die Verhältnisse der Spurenelemente haben. Deshalb sind die Ergebnisse dieser beiden Proben kritisch zu betrachten. Die Zr/Sc Datenpunkte des Conlara Metamorphic Complex sind in Bezug auf das Recycling ähnlich denen des Nogolí Metamorphic Complex (12 - 29), allerdings sind hier die Th/Sc Daten zwischen 0.7 und 1.4 etwas geringer. In der San Luis Formation liegt eine gute Korrelation zwischen $\mathrm{Th} / \mathrm{Sc}$ und $\mathrm{Zr} / \mathrm{Sc}$ Werten vor. Auffällig sind in dieser Einheit die phyllitische Probe A30-01 und die Arkose A12-06, die mit Zr/Sc Werten von 38 und 27 beide zu einem stärkeren Recycling tendieren als der Rest der dort genommenen Proben, die Verhältnisse von 13 - 20 aufweisen. In der Puncoviscana Formation sind die Unterschiede 


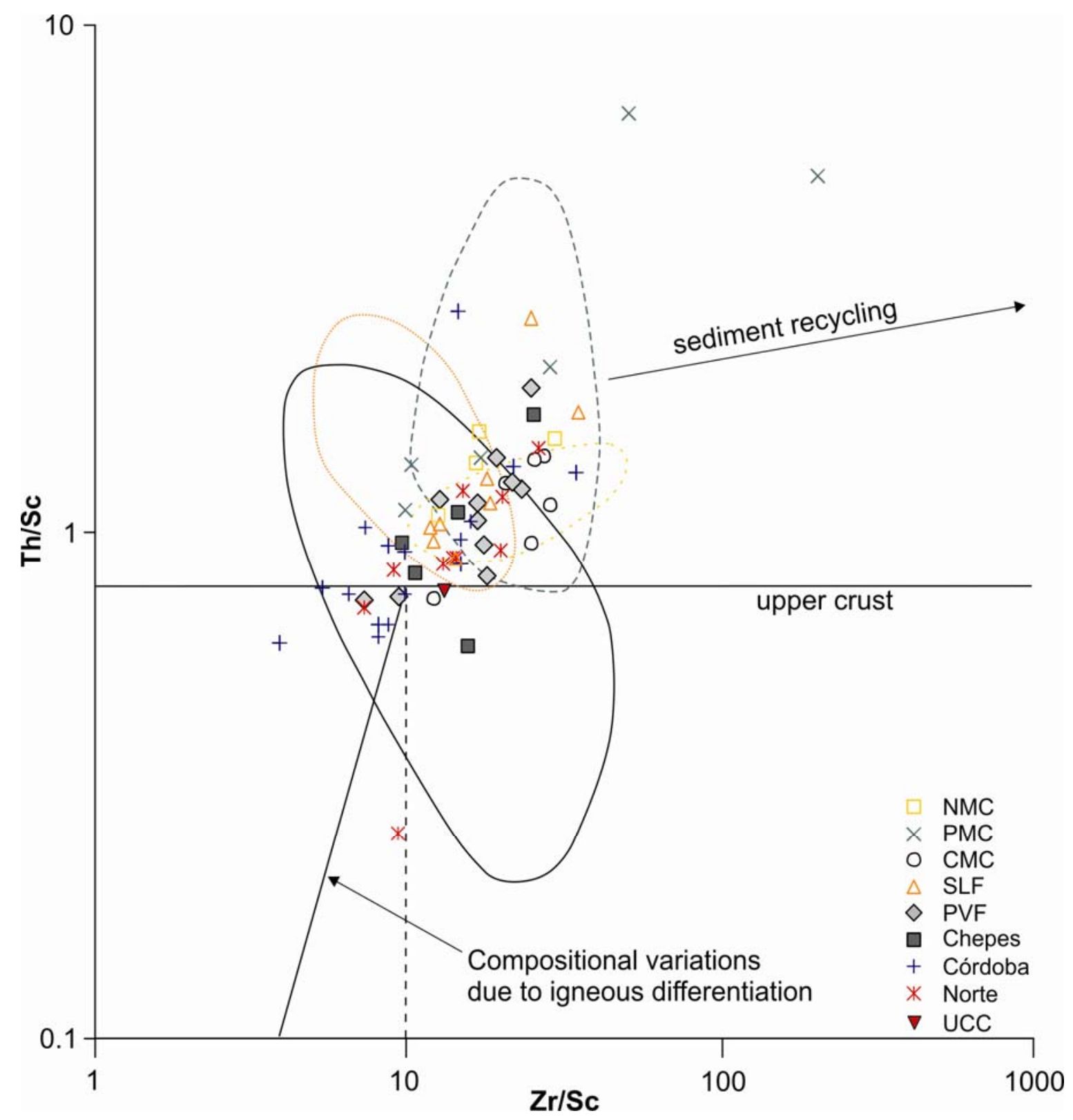

Abbildung 8: Th/Sc vs. Zr/Sc Diagramm nach McLennan et al. (1990) zur Ermittlung des Liefergebiets und dem Grad des Recycling der untersuchten (Meta)Sedimente der östlichen Sierras Pampeanas und der Puncoviscana Formation aus der Cordillera Oriental. Das Th/Sc Verhältnis gibt dabei den Anteil mafischen bzw. sauren Materials an und das $\mathrm{Zr} / \mathrm{Sc}$ Verhältnis ist ein Maß für die Aufarbeitung des Materials. Die Felder beziehen sich auf die Verteilung von Literaturwerten von Sims et al. (1998), Brogioni (2001), López de Luchi et al. (2003) und Zimmermann (2005). Lang gestrichelte Linie: Pringles Metamorphic Complex, durchgehende Linie: Conlara Metamorphic Complex, gepunktete Linie: San Luis Formation, kurz gestrichelte Linie: Puncoviscana Formation. Soweit möglich wurden die Felder in den Farben dargestellt, welche die Symbole der jeweiligen Einheiten besitzen.

des Recycling sehr gering. Wenn die beiden als Tonstein plottenden Proben A85-06 und A8606 mit ihren geringen $\mathrm{Th} / \mathrm{Sc}$ Werten von 0.7 und ihren ebenfalls geringen $\mathrm{Zr} / \mathrm{Sc}$ Daten von 10 und 8 ausgeklammert werden, liegen die übrigen neun Proben bei $\mathrm{Zr} / \mathrm{Sc}$ Verhältnissen von 14 - 25. Die Unterschiede sind hier eher durch die Th/Sc Daten gegeben. Einzig A88-06 liegt mit einem Th/Sc Wert von 1.2 und einem geringen $\mathrm{Zr} / \mathrm{Sc}$ Verhältnis von 14 etwas außerhalb der übrigen Datenpunkte. Die Metasedimente der Sierras de Chepes weisen $\mathrm{Zr} / \mathrm{Sc}$ 
Verhältnisse von 10 - 17 auf. Einzige Ausnahme ist A33-06 (28) mit einem ebenfalls erhöhten Th/Sc Wert von 1.7 und A37-06 mit einem Zr/Sc Verhältnis von 17, aber einem sehr niedrigen Th/Sc Verhältnis von 0.6. Die hochgradig metamorphen Gesteine der Sierras de Córdoba haben die geringsten $\mathrm{Zr} / \mathrm{Sc}$ Verhältnisse und liegen alle zwischen 5 und 10. Sie können allerdings durch ihre Th/Sc Daten in zwei Gruppen unterteilt werden, eine mit $\mathrm{Th} / \mathrm{Sc}$ Werten über 0.8 und eine mit Werten unter 0.8. Mittel- bis niedriggradig metamorphe Gesteine zeigen zwei klar unterscheidbare Populationen. Die mit den geringen $\mathrm{Zr} / \mathrm{Sc}$ Daten $<10$ überlappt die hochgradig metamorphen Proben. Die andere Gruppe hat $\mathrm{Zr} / \mathrm{Sc}$ Verhältnisse $>15$ und besteht aus dem Quarzphyllit A68-06, den gebänderten Gneisen A70-06 und A75-06, sowie der gneisigen Enklave aus dem San Carlos Diatexit A66-06 und A62-06, der als Rahmengestein des Achala Batholiths genommen wurde. All diese Gesteine haben $\mathrm{SiO}_{2}$ Werte von annähernd $70 \%$ oder mehr. In der Sierra Norte wurden drei Proben mit $\mathrm{Zr} / \mathrm{Sc}$ Werten von 8 - 10 genommen, von denen A81-06 einen extrem geringes $\mathrm{Th} / \mathrm{Sc}$ Verhältnis von 0.3 aufweist. Die übrigen Proben haben geringe $\mathrm{Zr} / \mathrm{Sc}$ Variationen und liegen zwischen 14 und 28 und zeigen die gleiche Tendenz zu lediglich geringem Recycling, wie die übrigen Einheiten. Bis auf zwei Proben des Pringles Metamorphic Complex (A13-06 und A19-06) deutet keine weitere Probe auf verstärkte Zirkonakkumulation, die auf verstärktes Recycling oder eine Sortierung der Sedimente zurückzuführen sein könnte, hin. McLennan et al. (1990) haben gezeigt, dass an aktiven Kontinentalrändern die Th/Sc Verhältnisse von Schlämmen unterschiedlich zu denen von Sanden aus dem gleichen Liefergebiet sein können. Allerdings konnte kein systematischer Unterschied in Bezug auf das $\mathrm{Zr} / \mathrm{Sc}$ Verhältnis gefunden werden. Der Unterschied bei den geringen Th/Sc Werten vor allem aus den Sierras de Córdoba dürfte vielmehr auf geringer entwickelte Magmen, bzw. einen größeren Einfluss dieser auf den Sedimenteintrag zurückzuführen sein (McLennan und Taylor 1991).

\subsubsection{Seltene Erdelemente (REE)}

Die Chondrit normierten (Taylor und McLennan 1985) REE Darstellungen (alle Elemente, die mit ${ }_{\mathrm{N}}$ beschriftet sind, beziehen sich auf Chondrit-normierte Verhältnisse; Bsp.: $\mathrm{La}_{\mathrm{N}}$ ) zeigen einen relativ ähnlichen Verlauf der unterschiedlichen Proben und Gebiete an (Abb. 9), die Gesamtkonzentration der REE kann jedoch deutlich schwanken. Im Nogolí Metamorphic Complex beträgt die REE Konzentration bei drei der vier Proben ca. 190 ppm, bei A16-06 allerdings lediglich 127 ppm. Die Steigung der Kurven, ausgedrückt in dem Chondritnormierten Verhältnis von $\mathrm{La}_{\mathrm{N}} / \mathrm{Yb}_{\mathrm{N}}$ beträgt bei A7-06 und A8-06 8.5 und 9.6, bei A39-01 und 
A16-06 allerdings lediglich 6.2. Der Grund hierfür ist aber bei beiden Proben unterschiedlich. Während der Verlauf der LREE, beschrieben durch das Verhältnis $\mathrm{La}_{\mathrm{N}} / \mathrm{Sm}_{\mathrm{N}}$ bei A39-01 bei 3.6 exakt im Bereich von A7-06 und A8-06 liegt, beträgt dieses bei A16-06 lediglich 2.7 und ist deutlich geringer als das der oberen kontinentalen Kruste (UCC = 4.4; Taylor und McLennan 1985) oder des post.Archean average Australian shale (PAAS = 4.2; Nance und Taylor 1976). Dies kann auf einen Verlust von LREE hinweisen, was auch die deutlich geringere Konzentration der $\Sigma$ REE erklären könnte. Bei A16-06 besteht der Grund für das geringe Verhältnis $\mathrm{La}_{\mathrm{N}} / \mathrm{Yb}_{\mathrm{N}}$ in der relativ hohen Konzentration der HREE, die deutlich über denen der restlichen Proben dieser Einheit liegen. Die $\mathrm{Eu} / \mathrm{Eu}^{*}$ Anomalie $\mathrm{Eu}_{\mathrm{N}} /\left(\mathrm{Sm}_{\mathrm{N}} * \mathrm{Gd}_{\mathrm{N}}\right)^{1 / 2}$, welche die Verteilung von Plagioklas angibt (hohe Werte weisen auf eine Anreicherung von Plagioklas hin, geringe auf eine Verarmung) liegt in dieser Einheit relativ konstant zwischen 0.53 und 0.61 und zeigt somit eine deutlich negative Eu/Eu* Anomalie an. Die Konzentration der REE im Pringles Metamorphic Complex ist stärker an die $\mathrm{SiO}_{2}$ Konzentration gebunden, die bei hohen Werten eine Verdünnung der Gesamtkonzentration bewirkt. Aus diesem Grund weisen die Proben A13-06, A18-06 und A19-06 ( $\left.\mathrm{SIO}_{2} 80-88 \%\right)$ geringe $\Sigma$ REE Konzentrationen von lediglich 170 ppm bzw. 150 ppm auf. Die übrigen Proben haben deutlich höhere Konzentrationen zwischen 210 und 280 ppm. Die $\mathrm{La}_{\mathrm{N}} / \mathrm{Yb}_{\mathrm{N}}$ Werte liegen zwischen 6.2 und 8.6, allerdings fallen für zwei Proben aus dem zentralen Bereich des Pringles Metamorphic Complex (A14-06 und A15-06) deutlich höhere Werte von 12.0 und 12.5 auf, die hauptsächlich auf eine für die jeweilige Probe geringe $\mathrm{Yb}$ Konzentration zurückgeht. Diese beiden Proben sind bereits durch ein für diese Einheit geringes $\mathrm{Th} / \mathrm{Sc}$ Verhältnis von 1.1 und 1.4 und ein geringes $\mathrm{Zr} / \mathrm{Sc}$ Verhältnis von 11 aufgefallen. Neben den hohen $\mathrm{SiO}_{2}$-Konzentrationen der Proben A13-06 und A19-06 könnten auch die in dieser Einheit geringsten Werte für $\mathrm{La}_{\mathrm{N}} / \mathrm{Yb}_{\mathrm{N}}$ von 6.2 und 7.3 ursächlich für die niedrigen Werte sein. Die Eu/Eu* Anomalie dieser Einheit ist stärker ausgeprägt als in den anderen Gebieten und beträgt mit Werten um 0.46 - 0.55 einen negativeren Charakter als die zuvor beschriebene Einheit. Die Verteilung der 5 REE liegt im Conlara Metamorphic Complex bis auf A10-06 einheitlich bei 150 - 170 ppm. Lediglich A10-06 besitzt einen deutlich höheren Wert von 240 ppm. Die $\mathrm{La}_{\mathrm{N}} / \mathrm{Yb}_{\mathrm{N}}$ Werte von 6.9 - 9.3 liegen in einem vergleichbaren Bereich zu den anderen untersuchten Gebieten, allerdings fällt auch hier eine Probe aus dem zentralen Gebiet wie im Pringles Metamorphic Complex mit einem $\mathrm{La}_{\mathrm{N}} / \mathrm{Yb}_{\mathrm{N}}$ Wert von 13.6 auf (A23-01). Auch dieser wird nicht durch einen steilen Verlauf der LREE hervorgerufen, sondern durch geringe HREE ausgelöst. Die $\mathrm{Eu} / \mathrm{Eu}^{*}$ Anomalie ist vergleichbar mit den übrigen beschriebenen Einheiten und liegt bei Werten zwischen 0.54 und 0.65. Die durchschnittliche 

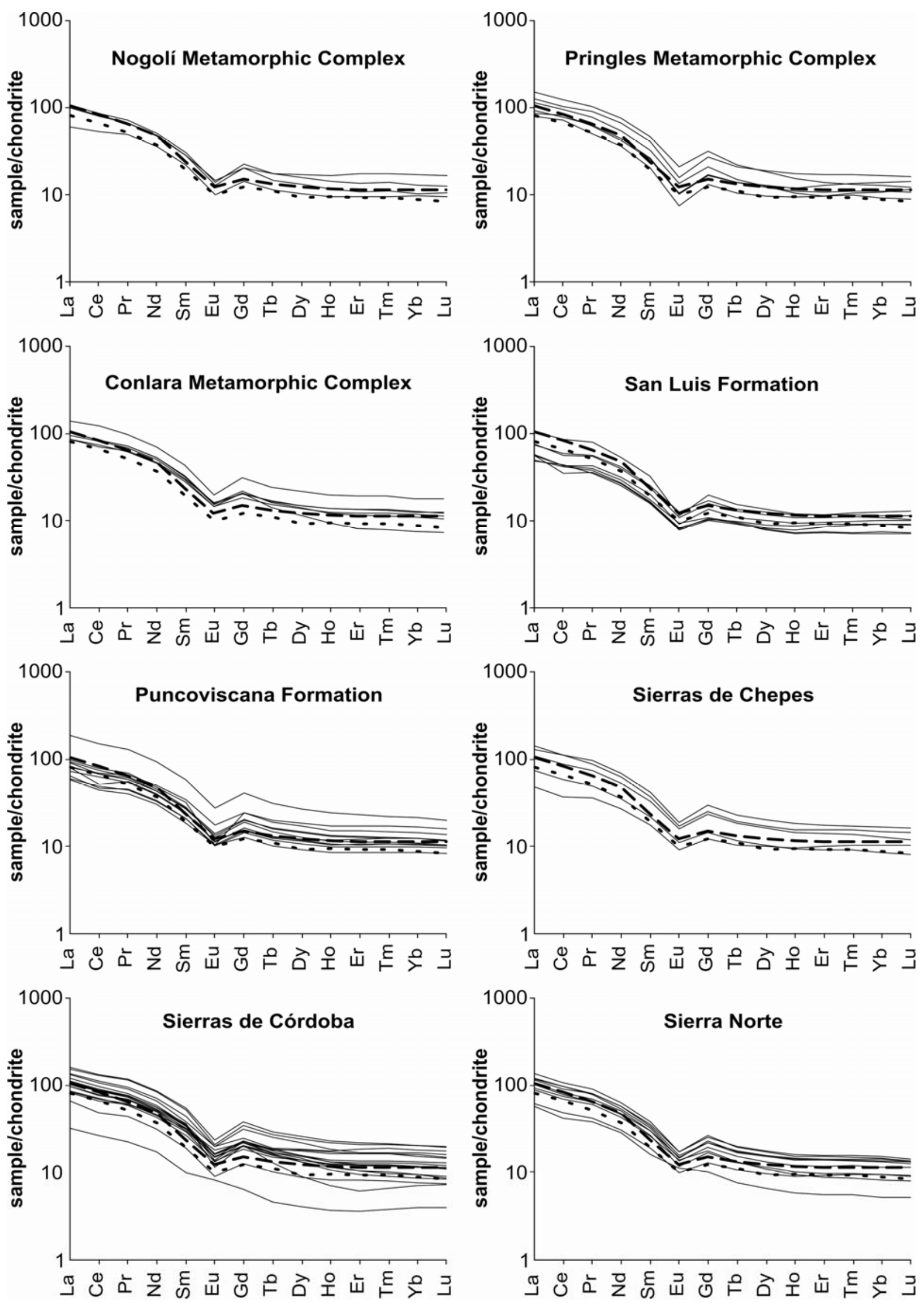

Abbildung 9: Chondrit normierte REE Verteilungsmuster (Taylor und McLennan 1985) für die östlichen Sierras Pampeanas und die Puncoviscana Formation aus der Cordillera Oriental. Die gestrichelte Linie gibt das REE Muster der oberen kontinentalen Kruste (Taylor und McLennan 1985) an. Die gepunktete Linie zeigt den Verlauf des post Archean average Australian shale (PAAS) nach Nance und Taylor (1976). 
Konzentration der $\Sigma$ REE der San Luis Formation ist mit 126 ppm am geringsten von allen untersuchten Einheiten. Lediglich A12-06 weist einen leicht erhöhten Wert von 200 ppm auf. Die geringen Werte gehen mit ebenfalls geringen Verhältnissen von $\mathrm{La}_{\mathrm{N}} / \mathrm{Yb}_{\mathrm{N}}$ einher, die bei 5.5 - 7.8 liegen. Auch hier liegt der Wert von A12-06 mit 8.5 am höchsten. Dies könnte auf eine generelle Mobilität der LREE hindeuten, oder aber es waren weniger Minerale verfügbar, die REE beinhalten. Die Eu/Eu* Anomalie liegt zwischen 0.55 und 0.68. Lediglich für die sehr $\mathrm{SiO}_{2}$ reiche Probe A12-06 (82\%) liegt dieser Wert mit 0.45 etwas geringer. Dies könnte mit dem stärkeren Recycling und der damit verbundenen Verwitterung von Plagioklas zusammenhängen. Die $\Sigma$ REE der Puncoviscana Formation beträgt $150 \mathrm{ppm}$ und liegt damit in etwa im Bereich der durchschnittlichen Werte der oberen kontinentalen Kruste (UCC, McLennan 1989). Lediglich der gebänderte Gneis A96-06 hat mit 350 ppm einen deutlich erhöhten Wert und auch ein hohes $\mathrm{La}_{\mathrm{N}} / \mathrm{Yb}_{\mathrm{N}}$ Verhältnis von 8.8. Die geringe Konzentration der REE könnte wie in der San Luis Formation mit niedrigen $\mathrm{La}_{\mathrm{N}} / \mathrm{Yb}_{\mathrm{N}}$ Verhältnissen zusammenhängen (5.7 - 8.5). Die Eu/Eu* Anomalie liegt in einem engen Bereich zwischen 0.55 und 0.60. Lediglich A87-06, mit $75 \%$ die $\mathrm{SiO}_{2}$ reichste Probe hat einen niedrigeren Wert von 0.38 und somit den negativsten Wert. A93-06 weist eine geringere Anomalie von 0.67 auf. Mit Ausnahme der Probe A96-06 mit ihren sehr hohen REE Konzentrationen bilden A87-06 und A93-06 die beiden Extreme mit den höchsten (A87-06) und den niedrigsten (A93-06) Konzentrationen an REE. In den Sierras de Chepes haben alle Proben bis auf A3706 den gleichen Verlauf der normierten REE. Die Proben A31-06, A33-06 und A35-06 haben $\Sigma$ REE Konzentrationen von 203 - 262 ppm, der niedriggradig metamorphe Psammit A41-06 dagegen weist lediglich $135 \mathrm{ppm}$ als $\Sigma$ REE auf. Den geringsten Wert findet man bei A37-06 mit 97 ppm. Dies hängt nicht mit einem Verdünnungseffekt wie teilweise im Pringles Metamorphic Complex zusammen $\left(\mathrm{SiO}_{2}\right.$ bei A37-06 = $60 \%$ ), könnte aber über die $\mathrm{La}_{\mathrm{N}} / \mathrm{Yb}_{\mathrm{N}}$ Verhältnisse erklärt werden. Diese liegen bei allen Proben der Sierras de Chepes bei 8.5 - 8.7, außer bei A37-06, wo dieser Wert lediglich 4.7 beträgt. Dies ist auch beim Wert $\mathrm{La}_{\mathrm{N}} / \mathrm{Sm}_{\mathrm{N}} \mathrm{zu}$ beobachten, der bei A37-06 lediglich bei 2.8 liegt, bei den anderen Proben dieser Einheit jedoch bei 3.4 - 3.5. Der niedrige Gehalt an $\Sigma$ REE könnte auch auf vulkanischen Eintrag zurückzuführen sein, da das Th/Sc Verhältnis bei lediglich 0.6 liegt, dem geringsten Wert der Proben aus den Sierras de Chepes. Ein solcher Eintrag wird über die Eu/Eu* Anomalie nicht angezeigt, die Werte liegen für alle Proben zwischen 0.53 und 0.63. In den Sierras de Córdoba haben die Proben A68-06 und A70-06 den höchsten Gehalt an $\Sigma$ REE von über 300 ppm. Diese beiden Gesteine haben auch, mit Ausnahme von A80-06, die höchsten Th/Sc Verhältnisse von 1.4 und 1.3 in diesem Gebiet. Beide Proben haben ein $\mathrm{La}_{\mathrm{N}} / \mathrm{Yb}_{\mathrm{N}}$ Verhältnis 
von 7.6 und 7.9, typisch für die obere kontinentale Kruste. Die zweite Gruppe hat $\mathrm{R} E E$ Konzentrationen um $250 \mathrm{ppm}$ und besteht aus den beiden tonalitischen Diatexiten A60-06 und A79-06 mit vergleichbaren $\mathrm{La}_{\mathrm{N}} / \mathrm{Yb}_{\mathrm{N}}$ Verhältnissen wie die oben genannten Proben, allerdings mit geringeren Th/Sc Verhältnissen von 1.0 und 0.8. Die dritte Gruppe besteht aus den Proben mit $\Sigma$ REE Konzentrationen von 180 - 220 ppm. Es sind sowohl mittelgradig metamorphe Gneise wie auch hochgradig metamorphe Stromatite, tonalitische und granitische Diatexite darunter. Eine Abgrenzung aufgrund der Gesteinsansprache ist demnach nicht möglich. Auch die Verhältnisse von $\mathrm{La}_{\mathrm{N}} / \mathrm{Yb}_{\mathrm{N}}$ schwanken deutlich zwischen 6.1 und 14.6. Ähnlich verhält es sich bei der vierten Gruppe mit den geringsten REE Konzentrationen von $120-165$ ppm. Auch hier zeigt sich eine hohe Bandbreite von verschiedenen Gesteinen. Das $\mathrm{La}_{\mathrm{N}} / \mathrm{Yb}_{\mathrm{N}}$ Verhältnis variiert allerdings etwas geringer zwischen 6.2 und 8.7. Die geringsten Verhältnisse mit 6.2 und 6.1 haben A61-06 und A77-06, beides hochgradig metamorphe Gesteine mit $\mathrm{Th} / \mathrm{Sc}$ Verhältnissen von $<0.7$. Dies könnte auf einen Verlust von LREE während der Metamorphose oder vulkanischen Einfluss schließen lassen. Die Eu/Eu* Anomalie liegt für die Sierras de Córdoba zwischen 0.49 und 0.67. Einzig A80-06 hat mit 0.42 eine etwas stärkere negative Anomalie. Diese Probe fällt zusätzlich durch ein sehr hohes Th/Sc Verhältnis von 2.7 und den steilsten Verlauf der REE mit einem $\mathrm{La}_{\mathrm{N}} / \mathrm{Yb}_{\mathrm{N}}$ Verhältnis von 14.6 auf. In der Sierra Norte können die Gesteine in eine Gruppe mit $\mathrm{La}_{\mathrm{N}} / \mathrm{Yb}_{\mathrm{N}}$ Werten von 6.5 - 8.4 (A101-06, A102-06, A103-06 und A108-06) und eine weitere Gruppe mit Werten von 8.9 - 11.1 unterteilt werden (A81-06, A100-06, A104-06, A105-06, A106-06 und A107-06). Die Proben A101-06, A102-06 und A103-06 wurden alle in einem Aufschluss genommen, in dem an einigen Stellen eine kontaktmetamorphe Überprägung, ausgelöst durch die Intrusion eines granitischen Körpers, zu beobachten war. Da die $\mathrm{La}_{\mathrm{N}} / \mathrm{Yb}_{\mathrm{N}}$ Verhältnisse aller drei in diesem Bereich genommenen Proben zu geringeren Verhältnissen neigen, kann eine Mobilisierung der LREE in diesem Bereich nicht ausgeschlossen werden. Die Probe A81-06 fällt durch einen unterschiedlichen Verlauf der REE auf (Abb. 9). Die Gesamtkonzentration der REE liegt bei lediglich 100 ppm und es liegt nur eine sehr schwach negativ ausgeprägte Eu/Eu* Anomalie von 0.88 vor (Rest der Sierras de Córdoba: 0.54 0.67). Die geringe Anomalie ist im Einklang mit dem sehr geringen $\mathrm{Th} / \mathrm{Sc}$ Verhältnis von 0.25. Beide Beobachtungen deuten auf einen vulkanischen Eintrag hin, der auch schon bei den Dünnschliffen vermutet worden war. 


\subsubsection{Geotektonische Position}

Bhatia und Crook (1986) haben gezeigt, dass das La/Th Verhältnis charakteristisch für die geodynamische Position sein kann. Demnach ist ein Verhältnis von $4.2 \pm 1.2$ typisch für einen ozeanischen Inselbogen (A), $2.4 \pm 0.3$ typisch für einen kontinentalen Inselbogen (B) und 1.8 \pm 0.1 für einen aktiven Kontinentalrand (C) und einen passiven Kontinentalrand (D). Die reinen Verhältnisse sind allerdings nicht allein ausschlaggebend, sondern auch die absoluten Konzentrationen der beiden Elemente, wobei besonders die Th-Konzentration vom ozeanischen Inselbogen über den kontinentalen Inselbogen zum passiven und aktiven Kontinentalrand ansteigt. Da sich das La/Th Verhältnis bei Sedimentationsprozessen konkordant verhält, ist es ein Charakteristikum des Liefergebietes. Im vorliegenden Diagramm (Abb. 10) ist deutlich zu erkennen, dass ein Großteil der Proben im Feld für kontinentalen Inselbogen oder in dessen Verlängerung liegt. Lediglich vier Proben liegen im Feld für aktiven und passiven Kontinentalrand. Dabei handelt es sich um die Probe A12-06, die im Diagramm von Herron (1988) eine Arkose ist (Abb. 4) und den höchsten Th/Sc Wert

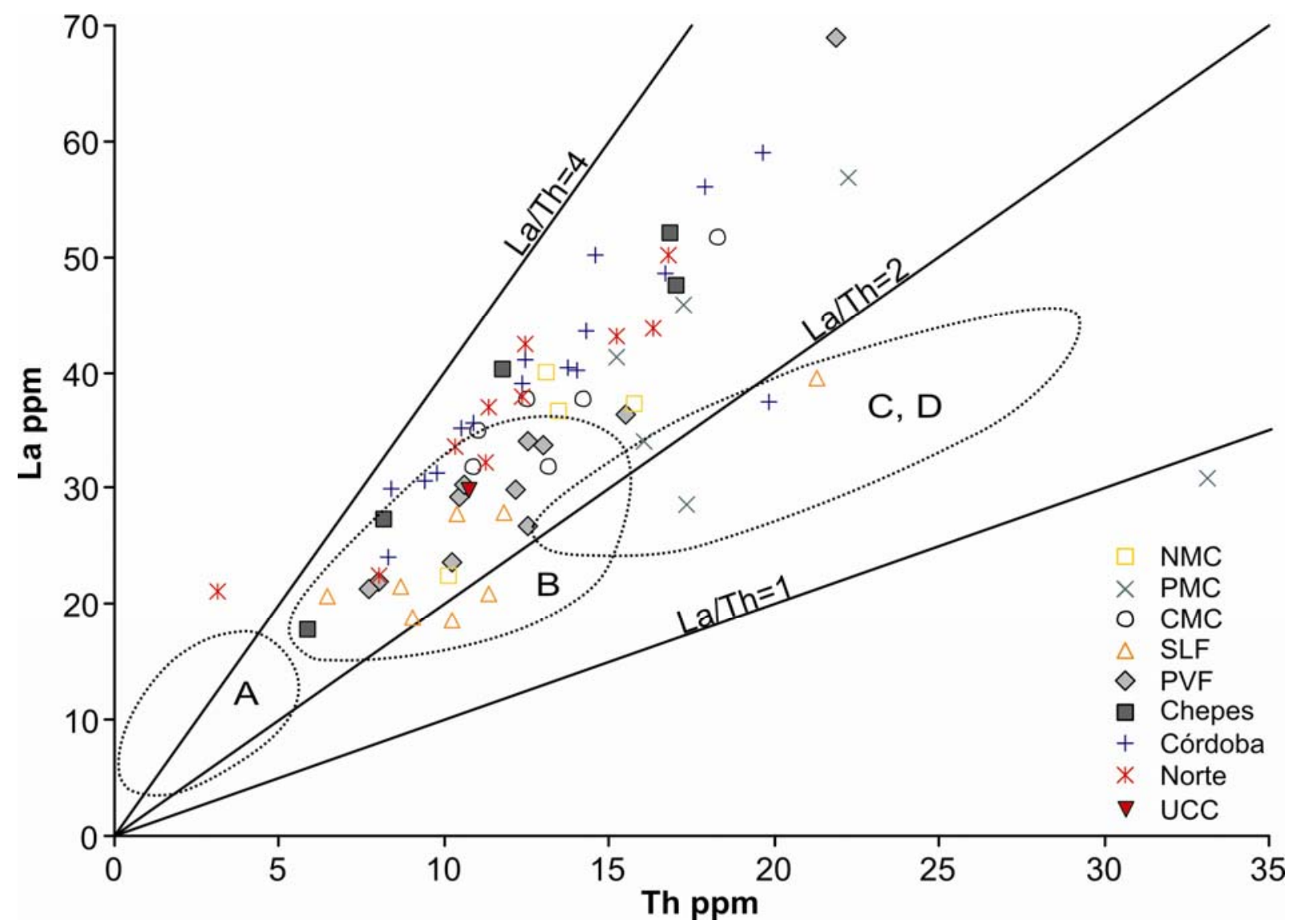

Abbildung 10: Diagramm zur Ermittlung der geotektonischen Position nach Bhatia und Crook (1986). Je niedriger das La/Th Verhältnis ist, desto stärker entwickelt ist die Probe. Aktiver und passiver Kontinentalrand können in diesem Diagramm nicht unterschieden werden. A: ozeanischer Inselbogen, B: kontinentaler Inselbogen, C: aktiver Kontinentalrand, D: passiver Kontinentalrand. 
der Sierra de San Luis mit 2.7 aufweist, den granitischen Diatexit A80-06 mit seinem für die Sierras de Córdoba sehr hohen Th/Sc Verhältnis von 2.7. Drei Proben aus dem Pringles Metamorphic Complex fallen ebenfalls durch niedrige La/Th Verhältnisse auf, von denen A18-06 und A19-06 im Bereich eines aktiven und passiven Kontinentalrandes liegen und A13-06 ein La/Th Verhältnis von 0.9 aufweist und außerhalb der vorgesehenen Felder liegt. In Bezug auf hohe La/Th Verhältnisse und geringe Gesamtkonzentrationen fällt die Probe A81-06 mit einem Verhältnis von 6.8 auf, doppelt so hoch wie der nächst niedrigere Wert.

Erhöhte Konzentrationen der Elemente Ti und Sc zeigen einen Eintrag von vulkanischem Material bzw. Material mit einer Mantelkomponente an, wohingegen hohe Werte von Zr und La die Wiederaufarbeitung sedimentären Materials widerspiegeln. Folglich kann in dem Diagramm Ti/Zr vs. La/Sc (Abb. 11) der Eintrag vulkanischen/basischen Materials auf der einen Seite und der Grad des Recycling gut verfolgt werden (Bhatia und Crook 1986). Bahlburg (1998) beanstandete an diesem Diagramm, dass nicht alle Proben, die im Feld für passiven Kontinentalrand liegen müssten, auch dort anzutreffen sein. Eine klare Tendenz zur Abgrenzung der einzelnen untersuchten Gebiete sollte allerdings trotzdem möglich sein. Es wurden keine Proben verwendet, die bei Herron (1988) als Tonstein charakterisiert wurden, weil deren Werte grundsätzlich deutlich über denen der Wacken und/oder Litharenite lag. Obwohl die meisten Proben nicht direkt in einem der Felder für die verschiedenen geotektonischen Milieus (Abb. 11) zu finden sind, fällt auf, dass sich die Proben des Nogolí Metamorphic Complex, des Conlara Metamorphic Complex, der San Luis Formation, der Puncoviscana Formation und den Sierras de Chepes in einem ähnlichen Bereich in der Nähe der Felder für kontinentalen Inselbogen und aktiven Kontinentalrand befinden. Einzige Ausnahme ist erstens der schwachmetamorphe Psammit A41-06 aus den Sierras de Chepes, der hohe Ti/Zr Werte aufweist. Dieser hat ein Th/Sc Verhältnis von 0.8 im Gegensatz zu 1.1 und 1.7 der anderen beiden Proben der Sierras de Chepes in diesem Diagramm. Die zweite auffällige Probe ist A81-06, die direkt neben dem Feld für ozeanischen Inselbogen liegt. Diese Probe hat das geringste Th/Sc Verhältnis der gesamten Kollektion von 0.25. Die Proben der Sierras de Córdoba haben höhere Ti/Zr Werte als alle anderen Einheiten. Auch die La/Sc Verhältnisse sind niedriger, wenn auch nicht so deutlich abweichend von den übrigen Gebieten. Zwölf von 14 Proben der Sierras de Córdoba haben Ti/Zr Werte über 40, was nach Bhatia und Crook (1986) auf einen ozeanischen Inselbogen hindeutet. Lediglich zwei Proben (A68-06 und A70-06) liegen darunter. Bei diesen beiden schwach- und mittelgradig metamorphen Gesteinen wurde bereits im Th/Sc vs. Zr/Sc Diagramm (Abb. 8) eine Tendenz zu erhöhtem Recycling beobachtet, was in Abbildung 11 bestätigt wird. Auch die 


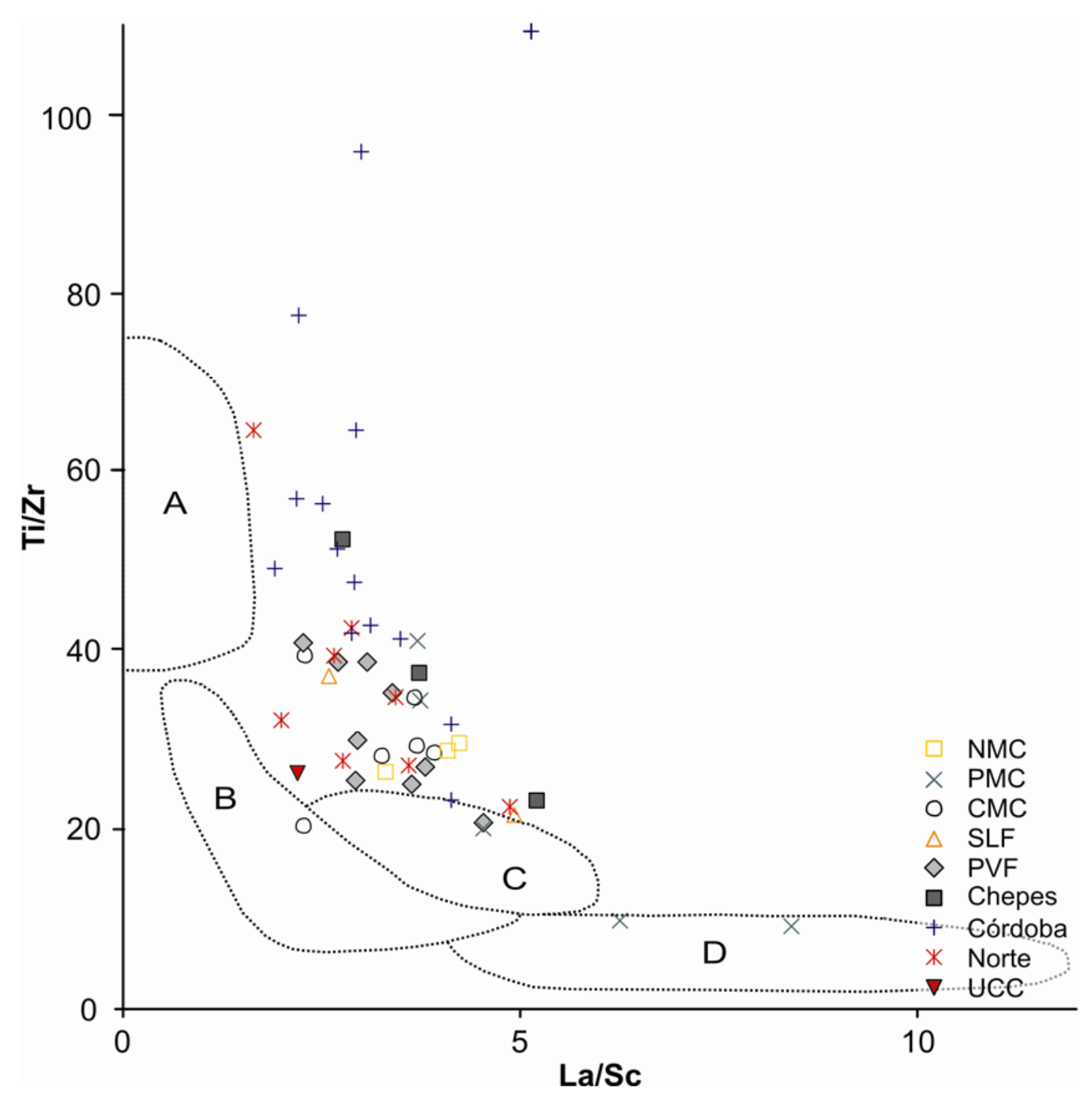

Abbildung 11: Diagramm zur Entschlüsselung der geotektonischen Position nach Bhatia und Crook (1986) für die Proben der östlichen Sierras Pampeanas und der Puncoviscana Formation der Cordillera Oriental, basierend auf dem Ti/Zr Verhältnis (hohe Werte sprechen für vulkanischen/mafischen Eintrag) aufgetragen gegen das La/Sc Verhältnis (hohe Werte deuten auf einen passiven Kontinentalrand hin). A: ozeanischer Inselbogen, B: kontinentaler Inselbogen, C: aktiver Kontinentalrand, D: passiver Kontinentalrand.

Probendaten des Pringles Metamorphic Complex verhalten sich ähnlich bimodal, wie in den vorangegangenen geochemischen Untersuchungen. Die Proben A13-06 und A19-06 liegen als einzige im Feld für einen passiven Kontinentalrand, während A5-01 und A14-06 zu leicht erhöhten Ti/Zr Werten tendieren. Neben den beiden Proben des Pringles Metamorphic Complex, die klar im Feld eines passiven Kontinentalrandes liegen, zeigen lediglich die Proben A12-06 (San Luis Formation), A18-06 (Pringles Metamorphic Complex), A33-06 (Sierras de Chepes), A87-06 (Puncoviscana Formation) und A104-06 (Sierra Norte) leichte Tendenzen einer stärkeren Wiederaufarbeitung, wenngleich sie im Diagramm im Bereich eines aktiven Kontinentalrandes liegen. Floyd und Leveridge (1978) haben gezeigt, dass durch die Darstellung von La/Th vs. Hf sehr gut die Liefergebiete wiedergeben werden können. Das La/Th Verhältnis ist dabei ein Maß für die mafischen/felsischen Komponenten, 


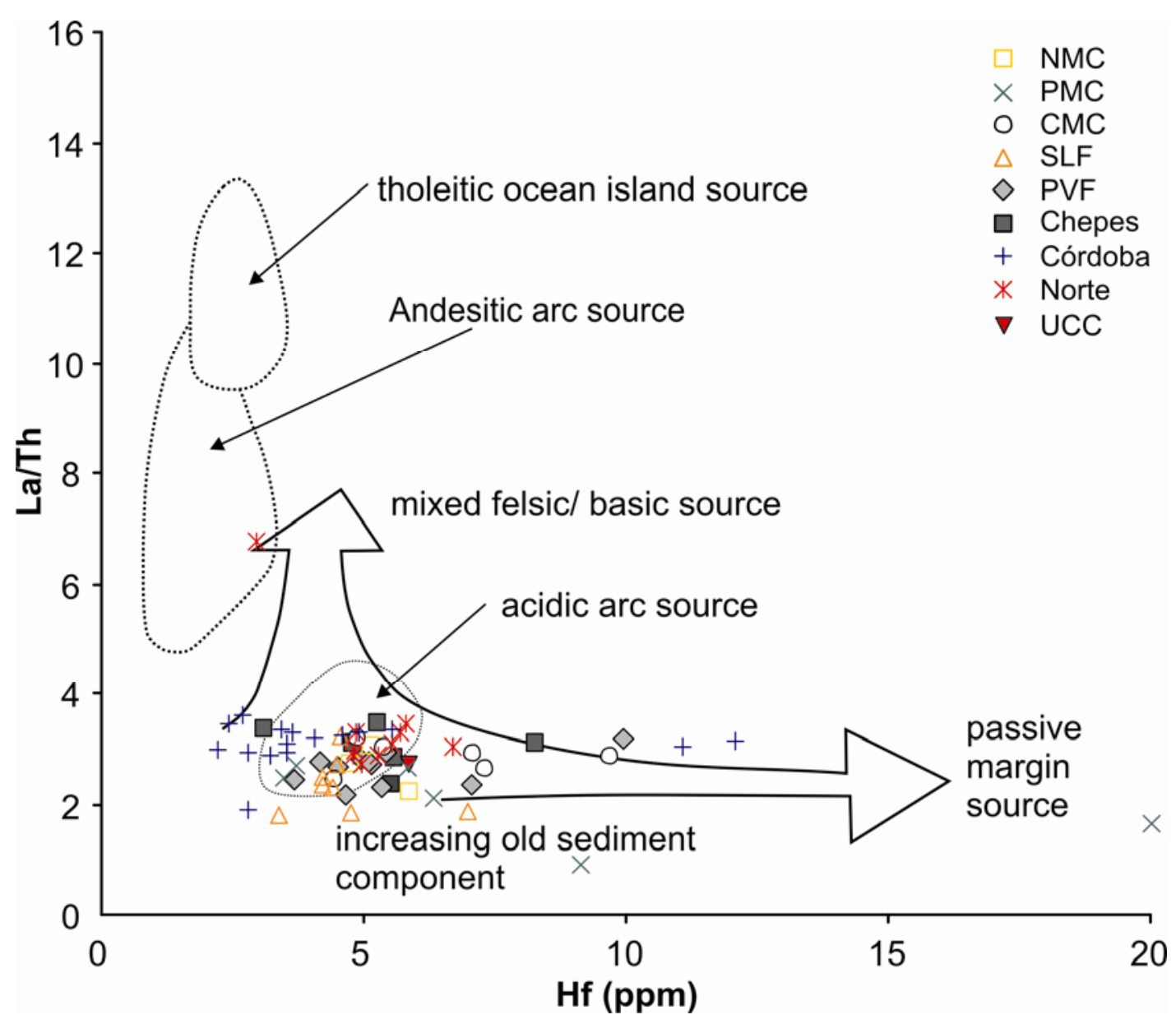

Abbildung 12: Diagramm zur Unterscheidung bestimmter geotektonischer Positionen nach Floyd und Leveridge (1987) für die Gesteine der östlichen Sierras Pampeanas und der Puncoviscana Formation der Cordillera Oriental. Hohe La/Th Verhältnisse deuten auf primitive Quellen hin, hohe Hf Konzentrationen sprechen für Recycling.

Hf bildet den Grad des Recycling ab, da es hauptsächlich aus dem Mineral Zirkon stammt. Das La/Th Verhältnis sinkt in stärker differenzierten Magmen, da Th inkompatibel ist und somit in der Restschmelze angereichert wird. Im Diagramm (Abb. 12) liegen die meisten Proben im oder in der Nähe des Bereichs, der von einem sauren Inselbogen zu erwarten wäre. Die La/Th Werte sind in einem Bereich zwischen 1.7 und 3.6. Ausnahmen bilden die vulkanisch beeinflusste Probe A81-06 $(\mathrm{La} / \mathrm{Th}=6.8)$ aus der Sierra Norte und A13-06 aus dem Pringles Metamorphic Complex $(\mathrm{La} / \mathrm{Th}=0.9)$. Die Hf Konzentrationen können größere Variationen aufweisen. Vier Proben des Pringles Metamorphic Complex liegen in der Nähe des Feldes für einen sauren Inselbogen mit Hf-Konzentrationen von 3.6 - 6.3 ppm, die Proben A13-06 und A19-06 weisen auf eine wesentlich stärkere Aufarbeitung hin und haben Hf Gehalte von 9.1 und 20 ppm. Der Nogolí Metamorphic Complex ist dagegen sehr homogen und die Hf-Werte schwanken zwischen 4.8 und 5.9 ppm. Die Werte des Conlara Metamorphic Complex weisen eine etwas größere Spanne von $4.4-7.3$ ppm auf und die schwach metamorphe Probe A10-06 zeigt den größten Wert in diesem Gebiet von 9.7 ppm. Sechs der acht Proben der San Luis Formation haben ein geringes Spektrum der Hf-Konzentration von 
4.2 - $4.6 \mathrm{ppm}$, A17-01 hat den geringsten Wert von $3.4 \mathrm{ppm}$ und die $\mathrm{SiO}_{2}$-reichste Probe A12-06 zeigt deutliche Anzeichen älterer sedimentärer Komponenten ( $\mathrm{Hf}=7.0 \mathrm{ppm})$. Diese Verteilung ist in der Puncoviscana Formation ähnlich (3.6 - 5.5 ppm). Zusätzlich existieren zwei Proben mit höheren Hf-Konzentrationen von 7.1 ppm (A81-06) und 9.9 ppm (A96-06). $\mathrm{Zu}$ A96-06 muss gesagt werden, dass diese Probe generell sehr hohe Konzentrationen an Spurenelementen aufweist (Anhang 1). Aus diesem Grund sollten bei dieser Probe geochemische Verhältnisse stärker bewertet werden, als absolute Konzentrationen. Vier Proben der Sierras de Chepes liegen bei einem Hf Gehalt um 5 ppm. A41-06, die bereits ein hohes Ti/Zr Verhältnis aufwies, hat einen geringeren Wert von 3.3 ppm und A33-06 mit dem geringsten $\mathrm{Ti} / \mathrm{Zr}$ Wert und dem höchsten $\mathrm{Th} / \mathrm{Sc}$ Verhältnis dieser Einheit weist jedoch Anzeichen stärkeren Recyclings auf und hat eine Hf Konzentration von 8.3 ppm. Die Proben aus den Sierras de Córdoba haben die geringsten Hf Werte $(2.4-4.0 \mathrm{ppm})$, lediglich mittelgradig metamorphe Gneise A62-06, A66-06 und A75-06 haben etwas höhere Werte von 4.6 - 5.6. Die beiden Proben A68-06 (Phyllit) und A70-06 (gebänderter Gneis) zeigen die größten Anzeichen stärker recyclierter Kruste (Hf $=11.0-12.0)$. Neben anderen charakteristischen Spurenelement-Plots, die Bhatia und Crook (1986) zur Liefergebietsbestimmung darstellten, wird in Abbildung 13 der Th-Sc-Zr/10 verwendet. Hohe $\mathrm{Zr} / \mathrm{Th}$ Werte zusammen mit niedrigen Sc Konzentrationen deuten auf einen passiven Kontinentalrand hin, wohingegen niedrige $\mathrm{Th} / \mathrm{Sc}$ Verhältnisse in Kombination mit niedrigen $\mathrm{Sc} / \mathrm{Zr}$ Werten auf einen ozeanischen Inselbogen schließen lassen. Im Th-Sc-Zr/10 Diagramm (Abb. 13) liegt nur eine Probe (A80-06) direkt im Feld für Sedimente an einem passiven Kontinentalrand. Weitere Proben, die in der Nähe dieses Feldes liegen, deuteten auch schon im La/Th vs. Hf Diagramm (Abb. 12) und anderen vorher beschriebenen Untersuchungen auf ein stärkeres Recycling der Sedimente hin, wie A19-06 aus dem Pringles Metamorphic Complex, A33-06 aus den Sierras de Chepes, A68-06 und A70-06 aus den Sierras de Córdoba und A87-06 aus der Puncoviscana Formation. Die in Abbildung 12 deutlich im Bereich zunehmender alter sedimentärer Komponente liegende Probe A13-06 aus dem Pringles Metamorphic Complex liegt in diesem Diagramm außerhalb des passiven Kontinentalrandes. Grund dafür ist der sehr hohe Th-Gehalt von 26 ppm. Dieser Wert wird nur noch von A12-06 aus der San Luis Formation mit 33 ppm übertroffen. Aufgrund dieses hohen Th Wertes liegen beide Proben im Feld für aktiven Kontinentalrand und nicht wie fast alle anderen Proben der gesamten Kollektion im Bereich eines kontinentalen Inselbogens. Auffällig ist, dass alle Proben des Pringles Metamorphic Complex zu hohen Th-Werten verschoben sind. Einzige Ausnahme ist A19-06, aufgrund der Zr Konzentration von 750 ppm, welche die Probe in 


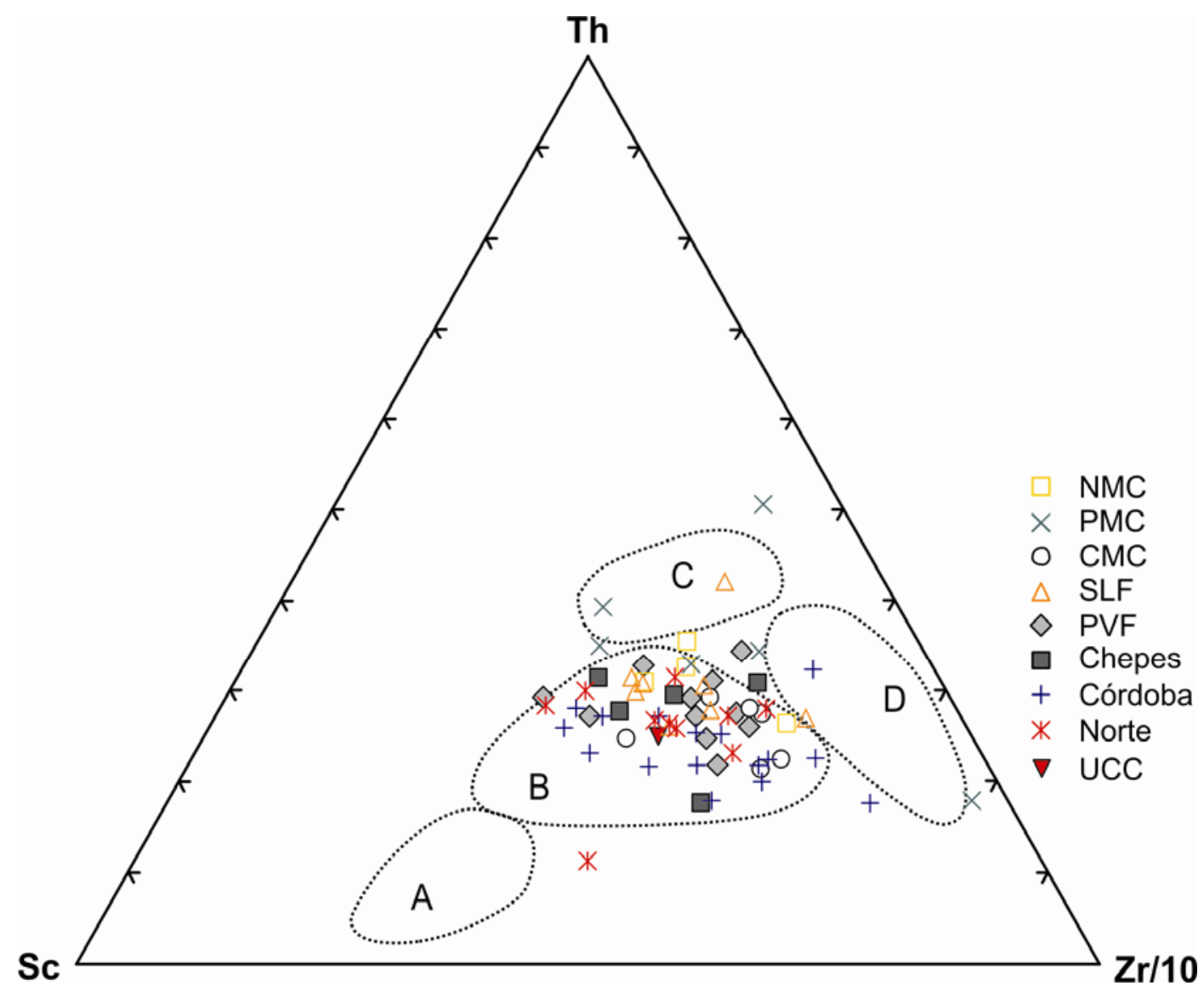

Abbildung 13: Diagramm zur Ermittlung der geotektonischen Position mit Hilfe der Th-Sc-Zr/10 Verhältnisse nach Bhatia und Crook (1986) für die Proben der östlichen Sierras Pampeanas und der Puncoviscana Formation aus der Cordillera Oriental. A: ozeanischer Inselbogen, B: kontinentaler Inselbogen, C: aktiver Kontinentalrand, D: passiver Kontinentalrand.

diesem Diagramm dominiert. Wie in den vorangegangenen Abbildungen liegt A81-06 abseits der übrigen Proben. Aufgrund des vulkanischen Charakters dieses Gesteins ist der Th Gehalt sehr niedrig, das $\mathrm{Zr} / \mathrm{Sc}$ Verhältnis ist allerdings zu groß, so dass die Probe nicht im Feld für einen ozeanischen Inselbogen liegt.

\subsection{Ergebnisse der Sm-Nd WR Isotopenanalysen}

Die Nd-Isotopie ist von Bedeutung für (meta)sedimentäre Gesteine, um die Modellalter $\left(\mathrm{T}_{\mathrm{DM}}\right)$

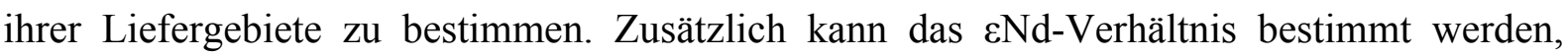
womit der Einfluss von Mantelgesteinen auf die Probe nachgewiesen werden kann. Je höher die Werte für $\varepsilon N d$ sind, desto stärker ist die Mantelkomponente, je niedriger, desto stärker ist der Einfluss des Liefergebietes und desto größer ist der sedimentäre Charakter des Gesteins. Mit Hilfe dieser Daten kann eine Charakterisierung möglicher Provenienzbereiche unterschiedlichen Alters und unterschiedlicher geologischer Geschichte vorgenommen werden. Die $\mathrm{T}_{\mathrm{DM}}$ Modellalter wurden nach dem Modell von Goldstein et al. (1984) berechnet, 
welches von einer linearen Entwicklung des Isotopensystems ausgeht. Bereits publizierte Daten wurden, wenn sie mit einem anderen Modell berechnet wurden, mit dem Modell von Goldstein et al. (1984) neu berechnet, um die Vergleichbarkeit zu gewährleisten. Die $\varepsilon N d$ Werte wurden auf 540 Ma zurückgerechnet, da dieses Alter die beste Näherung für die Sedimentation der Gesteine abbildet (Sims et al. 1998; Söllner et al. 2000; Steenken et al. 2006; Adams et al. 2006; Siegesmund et al. 2009), auch wenn der Pringles Metamorphic Complex, die San Luis Formation und die Sierras de Chepes möglicherweise etwas später sedimentiert wurden. Die Berechnungen der $\varepsilon \mathrm{Nd}_{(540 \mathrm{Ma})}$ und der $\mathrm{T}_{\mathrm{DM}}$ Daten sind in Abbildung 14 und 15 dargestellt. Die Proben haben ${ }^{147} \mathrm{Sm} /{ }^{144} \mathrm{Nd}$ Werte zwischen 0.110 und 0.127 . Lediglich A61-06 aus den Sierras de Córdoba hat einen etwas höheren Wert von 0.133 (Anhang 2). Diese geringe Spannweite der ${ }^{147} \mathrm{Sm} /{ }^{144} \mathrm{Nd}$ Verhältnisse deutet lediglich auf eine schwache Differenzierung während der Sedimentation, der Metamorphose und/oder Platznahme in die Kruste und ebenso nur auf eine geringe Fraktionierung der REE aufgrund exogener Prozesse hin (Jahn und Condie 1995). Dies wird auch durch den $f_{\mathrm{Sm} / \mathrm{Nd}}$ bestätigt $(\mathrm{f}=$ $\left[\left({ }^{147} \mathrm{Sm} /{ }^{144} \mathrm{Nd}\right)_{\text {heute }} /\left({ }^{147} \mathrm{Sm} /{ }^{144} \mathrm{Nd}\right)_{\mathrm{CHUR}}-1\right]$, dessen typische Werte zwischen -0.34 und -0.47 für

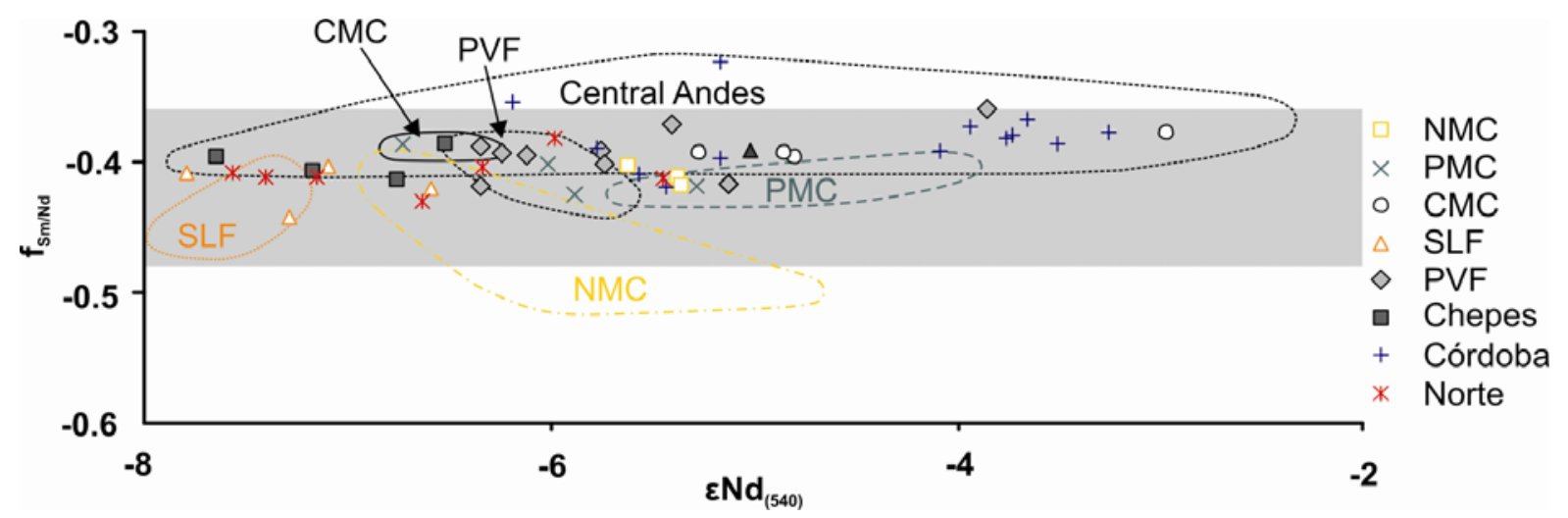

\begin{abstract}
Abbildung 14: Diagramm des $\mathrm{f}_{\mathrm{Sm} / \mathrm{Nd}}$ vs. $\varepsilon \mathrm{Nd}_{(540 \mathrm{Ma})}$ für die Proben der östlichen Sierras Pampeanas und der Puncoviscana Formation aus der Cordillera Oriental. Die eingezeichneten Felder beziehen sich auf Literaturwerte von Bock et al. (2000), Lucassen et al. (2000) und Steenken et al. (2004). Das graue Feld gibt die $\mathrm{f}_{\mathrm{Sm} / \mathrm{Nd}}$ Werte an, die typisch für die kontinentale Kruste sind (Goldstein et al. 1984).
\end{abstract}

Gesteine der kontinentalen Kruste liegt (Goldstein et al. 1984). Alle analysierten Proben liegen in diesem Bereich (Anhang 2).

Die drei untersuchten Proben des Nogolí Metamorphic Complex haben einheitliche $\mathrm{T}_{\mathrm{DM}}$ Werte zwischen 1.70 und $1.64 \mathrm{Ga}$ und $\varepsilon \mathrm{Nd}_{(540 \mathrm{Ma})}$ Verhältnisse zwischen -5.3 und -5.6. Im Pringles Metamorphic Complex liegt bei den vier untersuchten Proben eine größere Varianz bei den Modellaltern von $1.83-1.64 \mathrm{Ga}$ und ebenso ein größerer Unterschied der $\varepsilon \mathrm{Nd}_{(540 \mathrm{Ma})}$ Werte von -5.3 und -6.7 vor. Die Probe A13-06 $\left(87 \% \mathrm{SiO}_{2}\right)$ hat dabei das höchste $\mathrm{T}_{\mathrm{DM}}$ Modellalter von 1.83 Ga, wohingegen A14-06 und A15-06 mit 1.64 und 1.67 Ga deutlich 


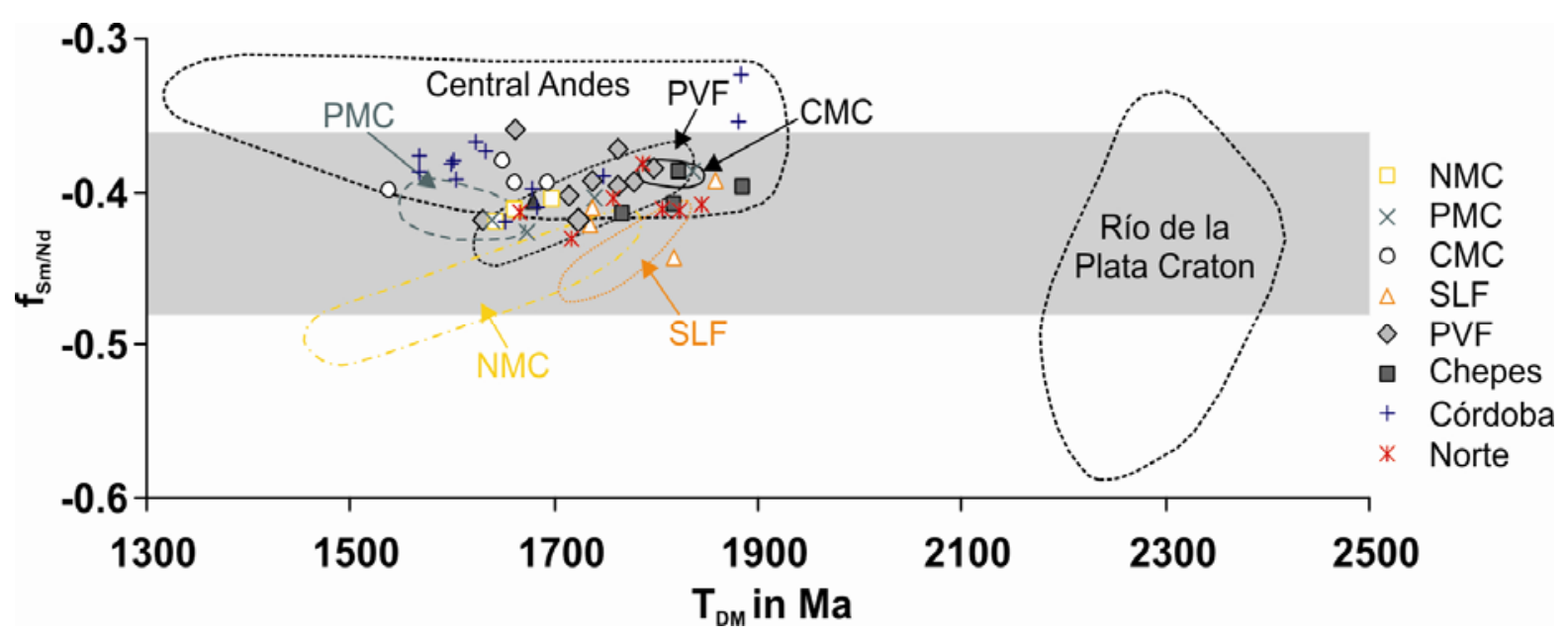

Abbildung 15: Diagramm des $\mathrm{f}_{\mathrm{Sm} / \mathrm{Nd}}$ vs. $\mathrm{T}_{\mathrm{DM}}$ für die Proben der östlichen Sierras Pampeanas und der Puncoviscana Formation aus der Cordillera Oriental. Bei den Proben und den Berechnungen wurden die gleichen Grundlagen wie in Abbildung 14 zugrunde gelegt, auch bei dem grauen Feld. Die Daten des Río de la Plata Kratons stammen von Iacumi et al. (2001) sowie Pankhurst et al. (2003). Die $\mathrm{T}_{\mathrm{DM}}$ Modellalter des Untersuchungsgebiets sind weit von denen des Río de la Plata Kratons entfernt.

jünger sind. Die zweite sehr $\mathrm{SiO}_{2}$ haltige $(84 \%)$ Probe $(\mathrm{A} 18-06)$ liegt mit ihrem $\mathrm{T}_{\mathrm{DM}}$ Modellalter von $1.75 \mathrm{Ga}$ dazwischen. Die jüngsten $\mathrm{T}_{\mathrm{DM}}$ Modellalter der Sierra de San Luis wurden im Conlara Metamorphic Complex gefunden. Drei der vier Proben haben mittlere Modellalter von 1.69 - $1.65 \mathrm{Ga}$ und $\varepsilon \mathrm{Nd}_{(540 \mathrm{Ma})}$ Werte zwischen -4.8 und -5.3. Die Probe A6105 weist mit $1.54 \mathrm{Ga}$ das jüngste $\mathrm{T}_{\mathrm{DM}}$ Modellalter metasedimentärer Gesteine der Sierra de San Luis auf und hat zudem ein hohes $\varepsilon \operatorname{Nd}_{(540}$ Ma) Verhältnis von -3. In der San Luis Formation haben vier der fünf gemessenen Proben $\varepsilon \mathrm{Nd}_{(540 \text { Ma) }}$ Werte von -6.6 bis -7.8 und $\mathrm{T}_{\mathrm{DM}}$ Modellalter von $1.86-1.74 \mathrm{Ga}$. Eine Probe (A30-01) liegt mit einem $\mathrm{T}_{\mathrm{DM}}$ Modellalter von $1.68 \mathrm{Ga}$ und einem $\varepsilon \mathrm{Nd}_{(540 \mathrm{Ma})}$ Verhältnis von -5.0 etwas außerhalb dieser vier Proben. In der Puncoviscana Formation liegen die $\varepsilon \mathrm{Nd}_{(540 \mathrm{Ma})}$ Daten zwischen -3.9 und -6.3 und die $\mathrm{T}_{\mathrm{DM}}$ Modellalter zwischen 1.80 und $1.63 \mathrm{Ga}(\mathrm{n}=9)$. Die niedriggradig metamorphen Metapelite A85-06 und A86-06, die unmittelbar westlich der Stadt Tucumán genommen wurden, haben dabei die geringsten $\mathrm{T}_{\mathrm{DM}}$ Modellalter von 1.66 und $1.63 \mathrm{Ga}$ und außerdem die höchsten $\varepsilon \mathrm{Nd}_{(540 \text { Ma) }}$ Daten von -3.9 und -5.1. Werden diese beiden Proben nicht berücksichtigt, so liegen die Daten der Puncoviscana Formation in einem sehr engen Bereich mit $\mathrm{T}_{\mathrm{DM}}$ Modellaltern von $1.80-1.72 \mathrm{Ga}$ und $\varepsilon \mathrm{Nd}_{(540 \mathrm{Ma})}$ Werten zwischen -5.4 und -6.3. Die vier Datenpunkte der Sierras der Chepes zeigen $\mathrm{T}_{\mathrm{DM}}$ Modellalter von $1.89-1.77 \mathrm{Ga}$ und $\varepsilon \mathrm{Nd}_{(540}$ Ma) Daten zwischen -6.6 und -7.7. Die Probe A37-06 hat mit 1.89 Ga und -7.7 das höchste $\mathrm{T}_{\mathrm{DM}}$ Modellalter und das negativste $\varepsilon \mathrm{Nd}_{(540}$ Ma) Verhältnis, obwohl dieses Gestein das geringste Th/Sc Verhältnis von 0.6 dieser Einheit aufweist, was auf mafische/vulkanische Einflüsse schließen lässt und das $\mathrm{T}_{\mathrm{DM}}$ Modellalter hätte senken und das $\varepsilon \mathrm{Nd}_{(540 \mathrm{Ma})}$ Verhältnis 
steigen lassen müssen. Die Sierras de Córdoba haben $\varepsilon \mathrm{Nd}_{(540 \mathrm{Ma})}$ Werte zwischen -3.2 und -6.2 sowie $\mathrm{T}_{\mathrm{DM}}$ Modellalter von $1.88-1.57 \mathrm{Ga}(\mathrm{n}=12)$. Unter den hochgradig metamorphen Proben befinden sich A80-06 aus dem Juan XXIII Diatexit (Abb. 3), der sich innerhalb des San Carlos Massivs befindet und der tonalitische Diatexit A61-06 aus dem südöstlichen Bereich der Sierras de Córdoba, der Sierra de Comechingones. Diese beiden Gesteine haben die höchsten $\mathrm{T}_{\mathrm{DM}}$ Modellalter von jeweils $1.88 \mathrm{Ga}$ und für die Sierras de Córdoba sehr geringe $\varepsilon \mathrm{Nd}_{(540 \mathrm{Ma})}$ Werte von -5.2 und -6.2. Diese beiden Analysen ergaben mit 0.133 und 0.127 die höchsten ${ }^{147} \mathrm{Sm} /{ }^{144} \mathrm{Nd}$ Verhältnisse aller Proben. Weiterhin liegt bei beiden Proben ein sehr geringes $\mathrm{La}_{\mathrm{N}} / \mathrm{Sm}_{\mathrm{N}}$ Verhältnis von lediglich 2.6 und 2.9 vor, so dass die Möglichkeit eines Verlustes von LREE in Betracht gezogen werden muss, was Auswirkungen auf das SmNd Isotopensystem nach sich ziehen könnte. Die übrigen hochgradig metamorphen Gesteine haben $\mathrm{T}_{\mathrm{DM}}$ Modellalter von $1.7-1.6 \mathrm{Ga}$ und $\varepsilon \mathrm{Nd}_{(540 \mathrm{Ma})}$ Werte von -3.7 bis -5.6. Die jüngsten Modellalter stammen von einem Stromatit (A77-06) und zwei tonalitischen Diatexiten aus dem San Carlos Massiv (A74-06 und A79-06). Unter den niedrig- bis mittelgradig metamorphen Gesteinen ist A66-06, ein gneisiger Bereich aus dem San Carlos Massiv die Probe mit dem höchsten Modellalter $(1.75 \mathrm{Ga})$ und dem geringsten $\varepsilon \mathrm{Nd}_{(540 \mathrm{Ma})}$ Wert (-5.8). Die übrigen drei Proben (A62-06, A68-06 und A70-06) haben geringe $\mathrm{T}_{\mathrm{DM}}$ Modellalter von $1.62-$ $1.57 \mathrm{Ga}$ und erhöhte $\varepsilon \mathrm{Nd}_{(540 \mathrm{Ma})}$ Verhältnisse zwischen -3.2 und -4.1. Aus der Sierra Norte wurden sechs Proben untersucht. Diese ergaben bis auf eine Ausnahme $\mathrm{T}_{\mathrm{DM}}$ Modellalter von $1.85-1.72 \mathrm{Ga}$ und $\varepsilon \mathrm{Nd}_{(540 \mathrm{Ma})}$ Werte von -6.0 bis -7.6. Das jüngste $\mathrm{T}_{\mathrm{DM}}$ Modellalter von 1.67 Ga stammt wie der höchste $\varepsilon \mathrm{Nd}_{(540 \mathrm{Ma})}$ Wert von -5.4 von der Probe A102-06. Diese Probe fällt durch einen extrem niedrigen $\mathrm{SiO}_{2}$ Gehalt von lediglich $47 \%$ und einem extrem ungewöhnlichen CIA Wert von 36 auf. Diese Daten lassen daran zweifeln, ob es sich bei dieser Probe um ein Metasediment bzw. eine Probe handelt, bei der keine Mobilisierung von Elementen auftrat. Die übrigen Proben haben ähnliche $\mathrm{T}_{\mathrm{DM}}$ Modellalter und $\varepsilon \mathrm{Nd}_{(540 \mathrm{Ma})}$ Verhältnisse. Dazu gehört auch A81-06 mit einem $\mathrm{T}_{\mathrm{DM}}$ Modellalter von 1.82 Ga und einem

$\varepsilon_{(540 \mathrm{Ma})}$ Wert von -7.4, obwohl dieses Gestein bei den geochemischen Untersuchungen häufig mafischen und vulkanischen Einfluss zeigt und ein Th/Sc Verhältnis von lediglich 0.25 besitzt (Abb. 7), was für einen deutlichen vulkanischen Eintrag spricht.

\subsection{Ergebnisse der Pb-Pb WR Isotopenanalysen}

Die Pb-Isotopie wurde bereits in verschiedenen Arbeiten eingesetzt, um unterschiedliche geologische Einheiten voneinander abzugrenzen (z.B. Aitcheson et al. 1995; Kay et al. 1996; 
Tosdal 1996; Bock et al. 2000; Lucassen et al. 2002; Loewy et al. 2004; Schwartz und Gromet 2004). Wenn lediglich ein homogenes Liefergebiet für die Schüttung der Sedimente zuständig war oder wenn mehrere Liefergebiete gut durchmischt wurden, sollte ein linearer Trend der Bleiisotope zu beobachten sein. Die Bleiverhältnisse spiegeln die durchschnittliche Isotopenzusammensetzung der metasedimentären Gesteine wider. Die Pb-Isotopie kann allerdings nicht nur zur Untersuchung der Liefergebiete herangezogen werden, sondern wird auch Auswirkungen, die nach der Sedimentation stattgefunden haben beinhalten. Die Zusammensetzung der Verhältnisse ${ }^{208} \mathrm{~Pb} /{ }^{204} \mathrm{~Pb},{ }^{207} \mathrm{~Pb} /{ }^{204} \mathrm{~Pb}$ und ${ }^{206} \mathrm{~Pb} /{ }^{204} \mathrm{~Pb}$ vergrößern sich durch den Zerfall von ${ }^{232} \mathrm{Th} \rightarrow{ }^{208} \mathrm{~Pb},{ }^{235} \mathrm{U} \rightarrow{ }^{207} \mathrm{~Pb}$ und ${ }^{238} \mathrm{U} \rightarrow{ }^{206} \mathrm{~Pb}$. Der Vorteil dieses Isotopensystems ist die Unabhängigkeit eines heutzutage auftretenden offenen Stoffsystems (Krogstad et al. 2004) bezüglich der Mutter- $\left({ }^{238} \mathrm{U},{ }^{235} \mathrm{U}\right.$ und $\left.{ }^{232} \mathrm{Th}\right)$ und Tochterisotope $\left({ }^{206} \mathrm{~Pb}\right.$, ${ }^{207} \mathrm{~Pb}$ und $\left.{ }^{208} \mathrm{~Pb}\right)$.

Die gegenwärtigen $\mathrm{Pb}-$ Verhältnisse im untersuchten Arbeitsgebiet liegen zwischen 15.63 und 15.89 für das ${ }^{207} \mathrm{~Pb} /{ }^{204} \mathrm{~Pb}$ Verhältnis, zwischen 18.35 und 20.66 für das ${ }^{206} \mathrm{~Pb} /{ }^{204} \mathrm{~Pb}$ Verhältnis und bei 38.59 - 40.69 für den Quotienten ${ }^{208} \mathrm{~Pb} /{ }^{204} \mathrm{~Pb}$ (Anhang 3). Im uranogenen Bleidiagramm (Abb. 16) zeigt sich ein linearer Trend zwischen den ${ }^{207} \mathrm{~Pb} /{ }^{204} \mathrm{~Pb}$ vs. ${ }^{206} \mathrm{~Pb} /{ }^{204} \mathrm{~Pb}$

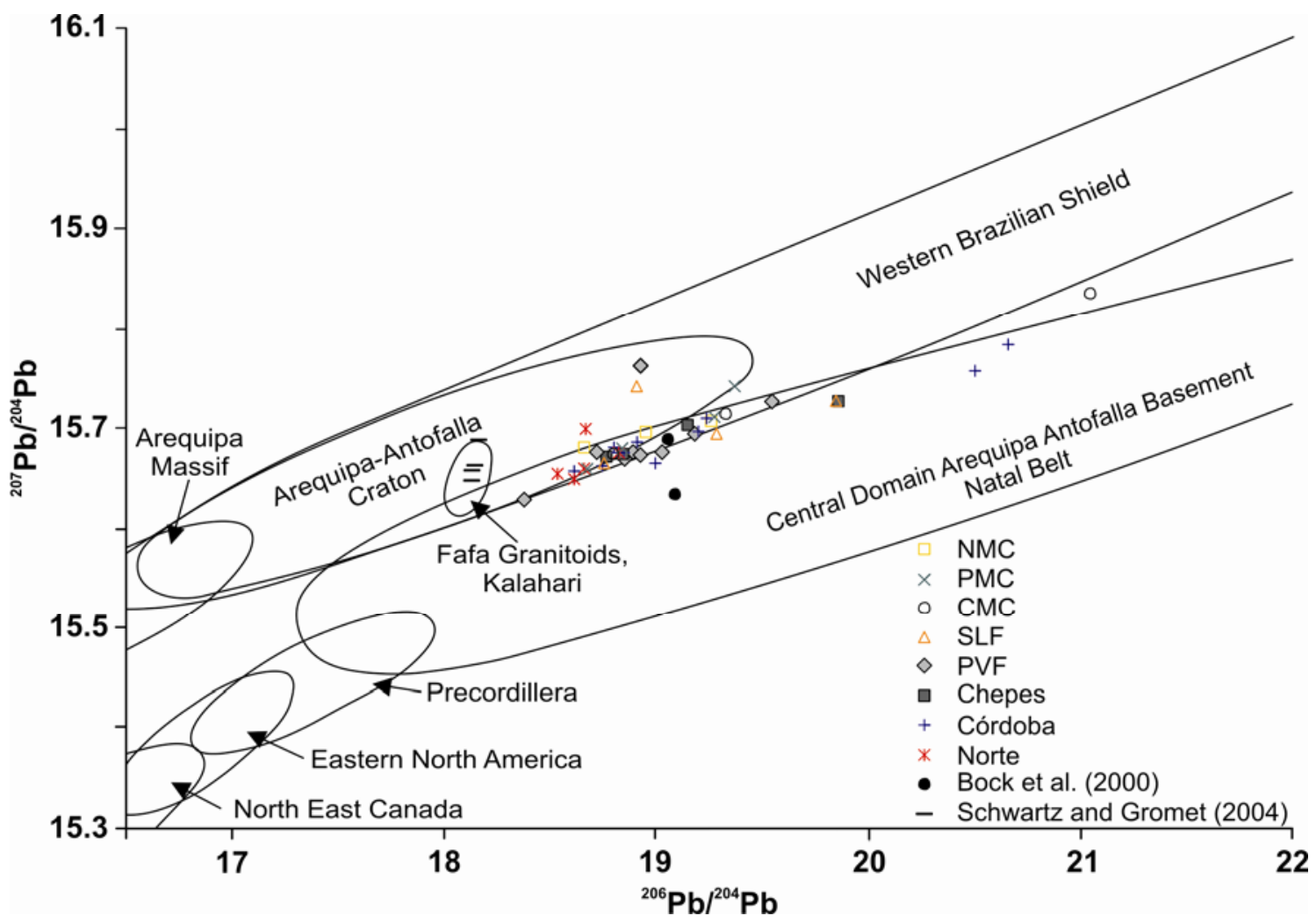

Abbildung 16: Diagramm der heutigen ${ }^{207} \mathrm{~Pb} /{ }^{204} \mathrm{~Pb}$ vs. ${ }^{206} \mathrm{~Pb} /{ }^{204} \mathrm{~Pb}$-Isotopie für das Untersuchungsgebiet. Die Felder der unterschiedlichen Gebiete stammen von Tosdal (1996). Die Datenpunkte liegen im Bereich derer von Bock et al. (2000) sowie Schwartz und Gromet (2004), die ebenfalls Metasedimente untersuchten. 
Werten. Lediglich der $\mathrm{SiO}_{2}$-reiche $(88 \%$ ) Gneis A13-06 aus dem Pringles Metamorphic Complex, die ebenfalls $\mathrm{SiO}_{2}$-reiche (82\%) Probe A12-06 aus der San Luis Formation, die psammitische Probe A88-06 aus der Puncoviscana Formation und die vulkanisch beeinflusste Probe A81-06 aus der Sierra Norte zeigen leicht erhöhte ${ }^{207} \mathrm{~Pb} /{ }^{204} \mathrm{~Pb}$ Werte an, liegen aber alle im Bereich der von Bock et al. (2000) für die Puncoviscana Formation und von Schwarz und Gromet (2004) beschriebenen Werte für die Sierras de Córdoba. Die Probe A10-06 aus dem Conlara Metamorphic Complex und die Proben A68-06 und A79-06 aus den Sierras de Córdoba weisen zwar deutlich höhere Werte für die ${ }^{207} \mathrm{~Pb} /{ }^{204} \mathrm{~Pb}$ und ${ }^{206} \mathrm{~Pb} /{ }^{204} \mathrm{~Pb}$ Verhältnisse auf, aber auch sie liegen auf dem linearen Trend der übrigen Proben.

Die Variationen im Diagramm ${ }^{208} \mathrm{~Pb} /{ }^{204} \mathrm{~Pb}$ vs. ${ }^{206} \mathrm{~Pb} /{ }^{204} \mathrm{~Pb}$ (Abb. 17) sind etwas größer als die des ${ }^{207} \mathrm{~Pb} /{ }^{204} \mathrm{~Pb}$ vs. ${ }^{206} \mathrm{~Pb} /{ }^{204} \mathrm{~Pb}$ Diagramms (Abb. 16). Auch wenn in diesem Diagramm (Abb.

17) bei den meisten Einheiten ein linearer Verlauf mit ähnlichen Isotopenzusammensetzungen beobachtet werden kann (Nogolí Metamorphic Complex, Conlara Metamorphic Complex, San Luis Formation, Puncoviscana Formation, Sierras de Córdoba), so fällt doch auf, dass die Werte des Pringles Metamorphic Complex leicht erhöht sind. In dieser Einheit ist weiterhin

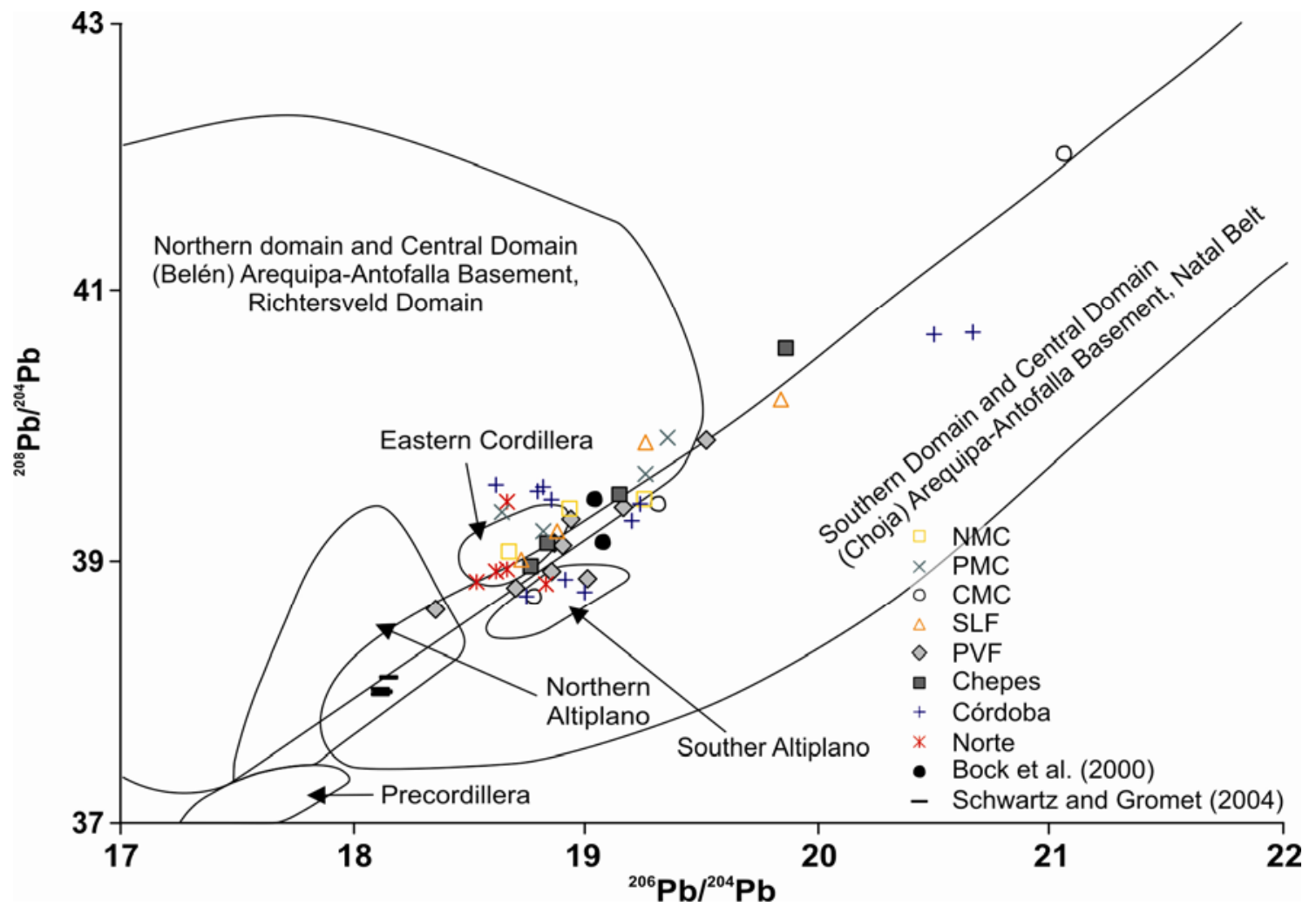

Abbildung 17: Diagramm der heutigen ${ }^{208} \mathrm{~Pb} /{ }^{204} \mathrm{~Pb}$ vs. ${ }^{206} \mathrm{~Pb} /{ }^{204} \mathrm{~Pb}$-Isotopie für das Untersuchungsgebiet. Die Felder der unterschiedlichen Gebiete stammen von Aitcheson et al. (1995), Kay et al. (1996), Loewy et al. (2003, 2004 und darin enthaltener Referenzen). Die Datenpunkte liegen im Bereich derer von Bock et al. (2000) sowie Schwartz und Gromet (2004), die ebenfall Metasedimente untersuchten. 
die metapelitische Probe A15-06 auffällig, da sie erhöhte ${ }^{207} \mathrm{~Pb} /{ }^{204} \mathrm{~Pb}$ Werte aufweist und etwas außerhalb der anderen Gesteine dieser Einheit liegt. Die Proben der Sierras de Córdoba zeigen bei den ${ }^{208} \mathrm{~Pb} /{ }^{204} \mathrm{~Pb}$ Verhältnissen die größten Unterschiede aller untersuchten Einheiten. Die Proben A60-06, A76-06, A78-06 und A80-06, alles hochgradig metamorphe Gesteine, besitzen erhöhte thorogene ${ }^{208} \mathrm{~Pb} /{ }^{204} \mathrm{~Pb}$ Werte. Die mittelgradig metamorphen Proben A62-06, A75-06 und A77-06 dagegen deuten auf ein verringertes ${ }^{208} \mathrm{~Pb} /{ }^{204} \mathrm{~Pb}$ Verhältnis hin und scheinen auf einem gemeinsamen Trend mit den ebenfalls mittelgradig metamorphen Gesteinen A66-06 und A70-06 zu liegen, die beide etwas höhere thorouranogene $\mathrm{Pb}$-Verhältnisse haben (Abb. 17). Die beiden Proben A68-06 (niedriggradig metamorph) und A79-06 (hochgradig metamorph) haben ähnlich wie A37-06 aus den Sierras de Chepes und A10-06 aus dem Conlara Metamorphic Complex hohe thorogene ${ }^{208} \mathrm{~Pb} /{ }^{204} \mathrm{~Pb}$ und ebenfalls hohe uranogene ${ }^{206} \mathrm{~Pb} /{ }^{204} \mathrm{~Pb}$ Verhältnisse. Allerdings liegen die beiden Proben aus den Sierras de Córdoba mitten im Feld der südlichen und zentralen Domain (Choja) des Arequipa-Antofalla Basements, sowie des Natal Belts (Abb. 17), wohingegen die anderen Proben mit erhöhten Pb-Verhältnissen am Rande oder leicht außerhalb dieses Feldes liegen. Ein im Vergleich zu den anderen Proben der Sierras de Córdoba leicht erhöhtes ${ }^{206} \mathrm{~Pb} /{ }^{204} \mathrm{~Pb}$ Verhältnis bzw. ein leicht verringertes ${ }^{208} \mathrm{~Pb} /{ }^{204} \mathrm{~Pb}$ Verhältnis wird deshalb nicht ausgeschlossen. In der Sierra Norte fällt eine Probe (A81-06), welche die größte vulkanische/mafische Komponente aufweist mit erhöhten ${ }^{208} \mathrm{~Pb} /{ }^{204} \mathrm{~Pb}$ Werten auf. Gegenteilige ${ }^{208} \mathrm{~Pb} /{ }^{204} \mathrm{~Pb}$ Verhältnisse sind bei A102-06 $\mathrm{zu}$ beobachten, die unterhalb des üblichen Trends für die Sierra Norte liegt. Aufgrund der kontaktmetamorphen Überprägung der Probe A102-06 könnte dies auf die Zufuhr von Uran hindeuten.

\subsection{Zirkon Geochronologie: U-Pb SHRIMP Datierungen}

SHRIMP U-Pb Datierungen wurden an insgesamt acht Proben der östlichen Sierras Pampeanas durchgeführt, sieben davon sind Metasedimente und eine Probe ist ein Orthogneis (A3-08). Die Proben A7-06 und A8-06 stammen aus dem Nogolí Metamorphic Complex, die Probe A73-05 wurde im Conlara Metamorphic Complex genommen, die Proben A30-01 und A93-05 wurden aus dem östlichen Phyllitband der San Luis Formation genommen, A1-08 und A4-08 stammen aus den Sierras de Chepes und der Orthogneis A3-08 wurde im Monte Guazú Complex, dem südlichsten Abschnitt der Sierra de Comechingones genommen. Alter unter $900 \mathrm{Ma}$ werden als ${ }^{206} \mathrm{~Pb} /{ }^{238} \mathrm{U}$ Alter diskutiert, bei Werten über $900 \mathrm{Ma}$ werden die 
${ }^{207} \mathrm{~Pb} /{ }^{206} \mathrm{~Pb}$ Alter herangezogen. Die Rohdaten der Analysen können im Anhang 4 nachgelesen werden.

Die Proben A7-06 und A8-06 wurden beide aus dem gleichen Aufschluss entlang der Straße von La Carolina nach San Francisco del Monte de Oro, einem nördlichen Querprofi des Nogolí Metamorphic Complex genommen. Aufgrund der Nähe der Probenahme und der Ähnlichkeit der Gesteine werden die Daten dieser Proben zusammen interpretiert. Beide sind graue, mittelkörnige metapsammitische Gneise mit den Mineralen Quarz + Biotit + Plagioklas + Muskovit als Hauptbestandteile. Akzessorisch treten in beiden Gesteinen Apatit und Zirkon auf. A7-06 beinhaltet zusätzlich etwas Klinozoisit. Die Proben gehören zu den bei von Gosen und Prozzi (1998) beschriebenen Ms-Bt/Bt-Ms Gneisen.

Die Zirkone (Abb. 18 a, b) zeigen unterschiedliche Formen, die von langprismatisch über kurzprismatisch bis $\mathrm{zu}$ gedrungen reichen und unterschiedliche Rundungen zeigen. Alle datierten Zirkone zeigen eine magmatische Zonierung in großen Teilen der jeweiligen Körner. Obwohl die langprismatischen Zirkone geringer zoniert sind, beinhalten sie immer noch eine komplexe geologische Geschichte mit ererbten Kernen und metamorphen Anwachssäumen. Es wurden 29 Datenpunkte an 22 Körnern gemessen, um die Provenienz und die metamorphe Geschichte des Nogolí Metamorphic Complex zu analysieren. Bis auf drei Ausnahmen liegen alle Th/U Verhältnisse zwischen 0.16 und 1.05, was auf ein magmatisches Wachstum dieser Bereiche hindeutet. Die drei übrigen Werte liegen zwischen 0.03 und 0.10 , was auf ein metamorphes Wachstum hindeutet. Elf der 29 Datenpunkte ergaben stark diskordante Werte (> $20 \%$ und/oder hohe Werte für gewöhnliches ${ }^{206} \mathrm{~Pb}(>1 \%)$ und werden nicht in die Diskussion eingehen. Zwei der verbliebenen 18 Analysen haben famatinische Alter (489 Ma und $501 \mathrm{Ma}$ ), elf ergeben pampeane Alter von 533 - $520 \mathrm{Ma}$, vier repräsentieren Ereignisse aus der Brasiliano Orogenese und liegen zwischen 730 und 600 Ma. Ein Alter von $1216 \mathrm{Ma}$ weist auf ein Ereignis der Grenvillian Orogenese hin. Unter Vernachlässigung der beiden ältesten pampeanen Datenpunkte (553 Ma und $552 \mathrm{Ma}$ ) ergeben die verbliebenen neun pampeanen Alter einen gewichteten Mittelwert von 528.5 \pm 4.8 Ma.

Bei A73-05 handelt es sich um einen gebänderten Gneis aus dem zentralen Gebiet des Conlara Metamorphic Complex. Die datierten Zirkone sind mäßig bis gut gerundet und ihre Internstruktur lässt bei den meisten Körnern auf mehrere geologische Ereignisse schließen (Abb. 18 c). Etwa $50 \%$ sind kurzprismatisch bis oblat, die anderen $50 \%$ sind langprismatisch. Einige Zirkone besitzen ältere, gerundete Kerne und beinhalten mindestens zwei weitere geologische Ereignisse (Abb. 18 c). Bis auf den Datenpunkt 1.2 (Anhang 4.1) liegen die Th/U Verhältnisse über 0.1. Bei dieser Probe wurden 26 Alter aus 21 Körnern 

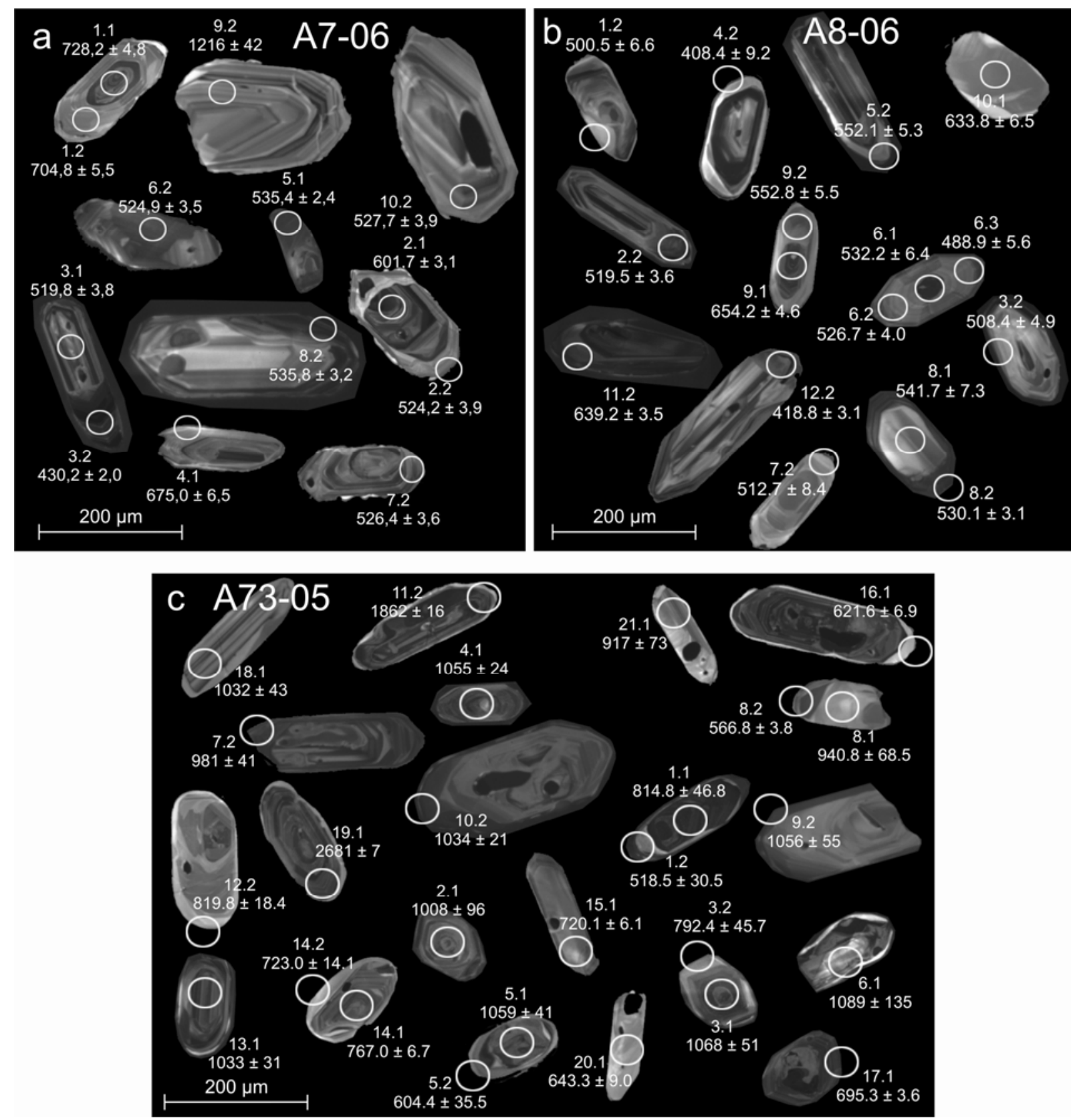

Abbildung 18: Kathodolumineszenz Bilder der Zirkone der Proben (a) A7-06 aus dem Nogolí Metamorphic Complex, (b) A8-06 aus dem Nogolí Metamorphic Complex und (c) A73-05 aus dem Conlara Metamorphic Complex. Die Kreise markieren die Analysepunkte. Die Werte geben das Alter der jeweiligen Analysen in $\mathrm{Ma}$ an. Die Alter sind ${ }^{206} \mathrm{~Pb} /{ }^{238} \mathrm{U}$ Alter, wenn sie unter $900 \mathrm{Ma}$ ergeben und ${ }^{207} \mathrm{~Pb} /{ }^{206} \mathrm{~Pb}$ Alter, wenn sie über $900 \mathrm{Ma}$ ergeben. Einige der vorliegenden Analysen gehen aufgrund zu hoher Diskordanzen und/oder zu hoher Konzentrationen an gewöhnlichem ${ }^{206} \mathrm{~Pb}$ nicht mit in die Diskussion ein.

ermittelt. Aufgrund stark diskordanter Daten und erhöhter ${ }^{206} \mathrm{~Pb}$ Werte wurden sieben Analysen von der Betrachtung ausgeschlossen. Von den verbleibenden 17 Altern ergibt der jüngste Datenpunkt $519 \pm 31$ Ma. Aufgrund seines geringen Th/U Verhältnisses könnte dieses Alter eine metamorphe Überprägung zu diesem Zeitpunkt widerspiegeln, allerdings besteht aufgrund einer Diskordanz von $12 \%$ (Anhang 4.1) auch die Möglichkeit, dass dieses Alter etwas zu niedrig ist, da das ${ }^{207} \mathrm{~Pb} /{ }^{206} \mathrm{~Pb}$ Alter deutlich höher ist und $581 \pm 52 \mathrm{Ma}$ ergibt. Bei dieser Probe ergibt sich kein pampeanes Maximum. Bis auf den Datenpunkt 1.2 liegen in 

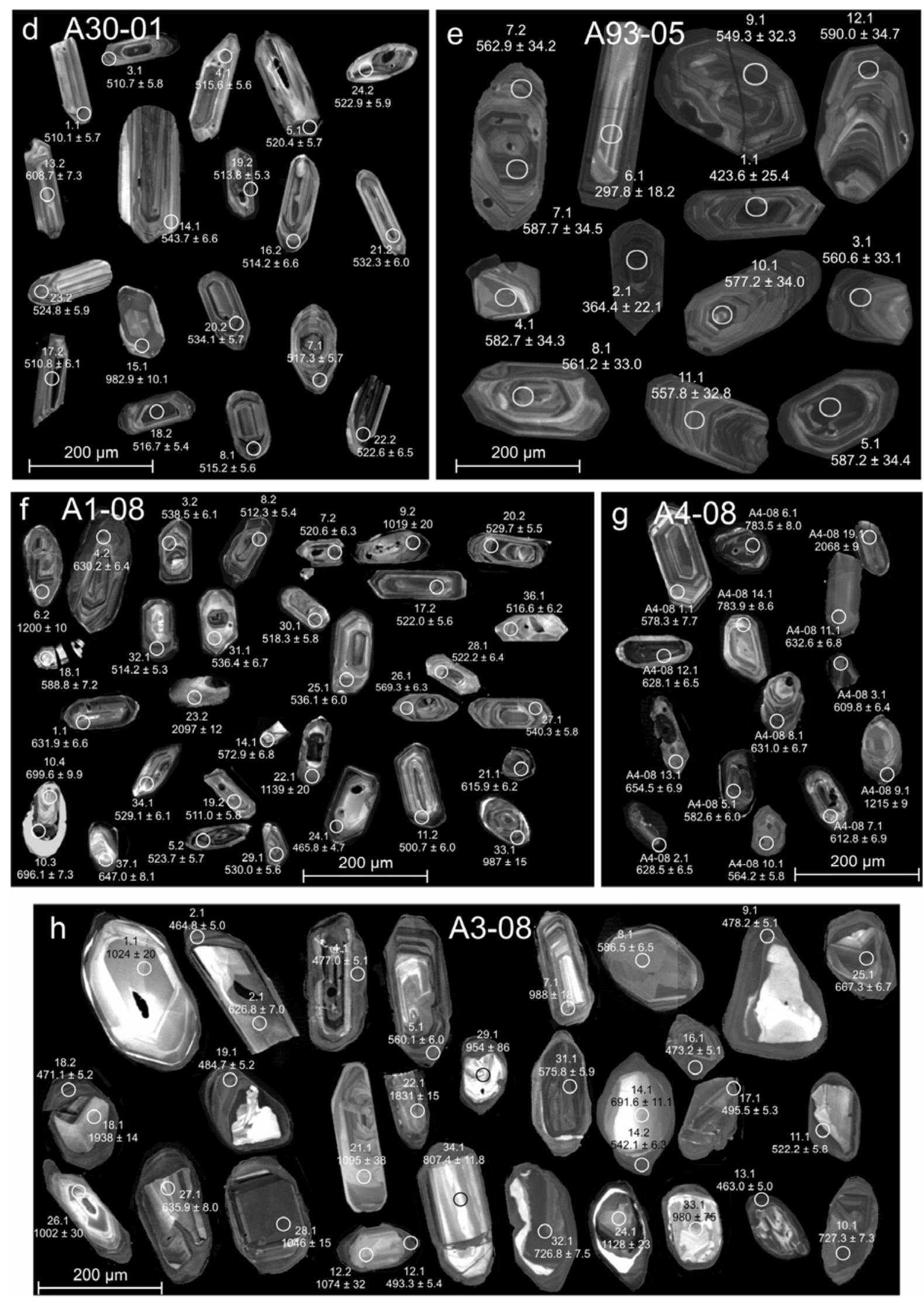

Abbildung 18: Kathodolumineszenz Bilder der Zirkone (d) A30-01, (e) A93-05, beide aus der San Luis Formation, (f) A1-08, (g) A4-08, beide aus den Sierras de Chepes und (h) A3-08 aus dem südlichsten Bereich der Sierras de Córdoba. Die Kreise markieren die Analysepunkte. Die Werte geben das Alter der jeweiligen Analysen in Ma an. Die Alter sind ${ }^{206} \mathrm{~Pb} /{ }^{238} \mathrm{U}$ Alter, wenn sie unter 900 Ma ergeben und ${ }^{207} \mathrm{~Pb} /{ }^{206} \mathrm{~Pb}$ Alter, wenn sie über $900 \mathrm{Ma}$ ergeben. 
dieser Probe keine pampeanen Alter vor. Es ergibt sich ein allmählicher Anstieg der Werte zwischen 600 und $815 \mathrm{Ma}(\mathrm{n}=5)$. Diese Alter wurden sowohl an den Rändern als auch im Zentrum der Zirkone gemessen. Die ältere Gruppe liegt bei Altern zwischen 1056 und 917 Ma $(n=9)$, einem für die Grenvillian Orogenese typischen Bereich. Auch diese Alter stammen sowohl von Randbereichen als auch aus den Kernen der detritischen Zirkone. Zwei Alter ergeben paläoproterozoische (1860 Ma) und neoarchaische Alter (2680 Ma).

Die Proben A30-01 und A93-05 stammen beide aus der San Luis Formation. Es handelt sich jeweils um feinkörnige schwach metamorphe Gesteine mit einem hohen Anteil an Phyllosilikaten, den typischen Phylliten der San Luis Formation.

Bei A30-01 wurden von 26 Altern sieben aufgrund zu hoher ${ }^{206} \mathrm{~Pb}$ Werte und/oder zu hoher Diskordanz (Anhang 4.2) nicht verwendet (2.1, 9.1 10.1 11.2, 12.1, 25.4, 26.2), so dass in dieser Probe nur Daten mit einer Diskordanz unter $10 \%$ diskutiert werden. Die Zirkone sind sowohl lang- als auch kurzprismatisch und zeigen alle eine magmatische Zonierung (Abb. 18 d), sind gerundet, in einigen Fällen jedoch zerbrochen. Von den 19 in der Diskussion verwendeten Daten liegen 16 in einem Bereich von 544 - 510 Ma (Abb. 19 c, 20 c, c'). Aus diesen Analysen wurden fünf Punkte verwendet (3.1 5.1, 7.1, 16.2, 18.2) und ergaben ein konkordantes Alter (Abb. 20 c'") von $516.3 \pm 2.7$ Ma $(M S W D=2.1,2 \sigma)$. Lediglich drei Analysen lagen nicht in dem pampeanen Maximum. Zwei Datenpunkte ergaben Alter von $600 \mathrm{Ma}$ und $609 \mathrm{Ma}$, typisch für die Brasiliano Orogenese und ein Zirkon ergab ein Grenvillian Alter von 982 Ma.

Die Probe A93-05 stammt ebenfalls aus der San Luis Formation. Die verwendeten Zirkone sind im Gegensatz zu A30-01 ähnlich gerundet und auch zoniert, allerdings sind sie deutlich größer (Abb. 18 d). Aufgrund sehr hoher diskordanter Werte (> $500 \%$, Anhang 4.2) wurden drei Datenpunkte nicht verwendet. Die restlichen zehn ergeben ein sehr enges Spektrum zwischen 590 und $550 \mathrm{Ma}$ (Abb. 19 d, 20 d, d') mit einem Mittelwert von etwa 570 Ma. Bis auf die extrem diskordanten Datenpunkte gibt es kein Alter außerhalb dieses Altersbereichs, ähnlich wie bei A30-01. Der einzige Unterschied besteht in dem geringeren Alter der Zirkone von knapp unter 520 Ma (A30-01) im Gegensatz zu den 570 Ma der Probe A93-05. Auffällig ist der Datenpunkt 14.1 der Probe A30-01 (Abb. 18 e). Dieser stammt aus dem mit Abstand größten Zirkon aus Probe A30-01 (ähnlich der Größe der Zirkone aus A93-05), ergibt das höchste pampeane Alter von 544 Ma und kommt damit sehr nah an die Alter aus Probe A9305 heran.

Die Proben A1-08 und A4-08 wurden in den Sierras de Chepes genommen. Es handelt sich bei beiden Gesteinen um gebänderte Gneise, die aus Biotit und Quarz-Plagioklas-Lagen 
aufgebaut werden. Der Metamorphosegrad, die Korngröße und die Bänderung sind vergleichbar mit den Eigenschaften der Probe A35-06, die ebenfalls in den Sierras de Chepes genommen wurde. Im Dünnschliff ist keine Faltung der Bänderung sichtbar. Diese ist im Handstück jedoch sehr gut zu erkennen. Die Zirkone kommen meistens in Biotitkristallen vor, können jedoch auch vereinzelt isoliert in Quarzkörnern auftreten. Beide Proben wurden als Xenolithe im Chepes Granodiorit genommen.

Aus der Probe A1-08 wurden 37 Alter aus 36 Zirkonen berechnet (Abb. 18 f). Vier Alter wurden nicht verwendet $(13.2,15.2,16.2,12.2)$, da sie zu diskordant waren oder zu hohe Gehalte an gewöhnlichem ${ }^{206} \mathrm{~Pb}$ enthielten. Das jüngste Alter wurde mit $465.8 \pm 4.7 \mathrm{Ma}$ berechnet. Die Diskordanz dieses Datenpunktes liegt bei $8 \%$. Aus dieser Analyse ergibt sich ein ${ }^{207} \mathrm{~Pb} /{ }^{206} \mathrm{~Pb}$ von $506 \mathrm{Ma}$. Das nächst jüngere Alter liegt bei $500 \mathrm{Ma}$, gefolgt von einer Gruppe aus 14 Datenpunkten von 540-511 Ma mit einem durchschnittlichen Alter von $524 \pm$ $6 \mathrm{Ma}$. Wenn die vier ältesten Punkte herausgelassen werden, sinkt der Durchschnittswert auf $518 \pm 4$ Ma. Aus den Datenpunkten $(7.2,15.2,17.2,36.1)$ lässt sich ein konkordantes Alter von $520.5 \pm 3.1 \mathrm{Ma}(\mathrm{MSWD}=3.1,2 \sigma)$ berechnen (Abb. 20 e'"), das ein geologisches Ereignis im Liefergebiet der Sierras de Chepes datiert. Solche Maxima wurden bereits von Adams et al. (2008) für einige Bereiche der Puncoviscana Formation und Collo et al. (2009) für detritische Zirkone im Famatina Belt (Abb. 1) berichtet. Die letztgenannten Autoren erhielten ein Maximum um 520 Ma und ein weiteres um 640 Ma in zwei Proben ihres Untersuchungsgebietes. In Probe A1-08 ergibt sich ein weiters spätpampeanes Maximum etwas geringerer Intensität um $570 \mathrm{Ma}$, gefolgt von zwei Maxima, die Aktivitäten während der Brasiliano Orogenes um 630 und 700 Ma abbilden. Es folgt eine Lücke bis zu Altern von 1200 - 1000 Ma. Außerhalb dieses Bereichs liegt lediglich eine Analyse, die bei $2100 \mathrm{Ma}$ liegt (Anhang 4.3).

Die Probe A4-08, ebenfalls ein gebänderter Gneis, wurde unweit von A1-08 genommen. Es wurden 19 Alter berechnet, von denen fünf nicht in die Diskussion miteinbezogen werden, da sie eine zu starke Diskordanz aufweisen, oder aber einen zu hohen Gehalt an gewöhnlichem ${ }^{206} \mathrm{~Pb}(4.1,15.1,16.1,17.1,18.1)$. Die jüngste Analyse (10.1, Anhang 4.3) ergibt ein Alter von $564 \mathrm{Ma}$, ist allerdings mit $10 \%$ relativ diskordant, sodass sich ein ${ }^{207} \mathrm{~Pb} /{ }^{206} \mathrm{~Pb}$ Alter über 600 Ma ergibt. Die anderen Daten ergeben eine Gruppe zwischen 650 und $580 \mathrm{Ma}(\mathrm{n}=9)$, zwei Alter um 780 Ma, ein Alter leicht über 1200 Ma und einen Datenpunkt um 2000 Ma. Dieses Alterspektrum zeigt klare Unterschiede zu der in unmittelbarer Nähe genommenen Probe A108 . 
Die Probe A3-08 ist ein Orthogneis aus dem Süden der Sierra de Comechingones. Es handelt sich um ein grobkörniges, hochgradig metamorphes Gestein mit ausgelängten Plagioklas und Biotit Kristallen und deutlich eingeregeltem Biotit. Die Zirkone liegen in den meisten Fällen als Einschlüsse in Biotit vor und sind nur selten außerhalb dieses Minerals zu finden. Es wurden 40 Alter berechnet, von denen sechs aufgrund zu hoher Diskordanz oder erhöhter ${ }^{206} \mathrm{~Pb}$ Konzentrationen verworfen werden mussten $(3.1,5.2,6.1,9.2,20.1)$. Die verbliebenen 34 Analysen haben ein starkes famatinisches Maximum (Abb. 18 h, 19g, 20 f, f'), mit einem

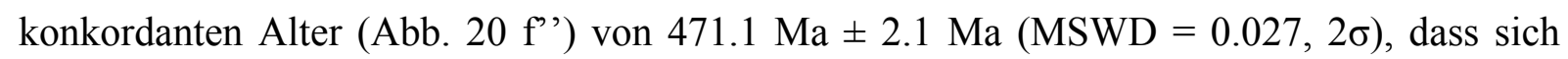
unter Verwendung der Analysepunkte (2.1, 4.1, 9.1 13.1, 16.1 18.2) berechnen lässt. All diese jungen Alter stammen aus Zirkonrändern mit extrem niedrigen Th/U Werten von 0.003 0.013, die auf ein metamorphes Wachstum dieser Bereiche schließen lassen. Die famatinischen Alter gehen sukzessive in pampeane bis frühpampeane Alter bis 580 Ma über. Die Th/U Verhältnisse dieser Alter sind mit Ausnahme von 8.1 (Anhang 4.4) mit Werten von $0.01-0.08$ immer noch gering, allerdings deutlich höher, als bei den famatinischen Altern. Nachfolgend schließen sich Brasiliano Alter bis $730 \mathrm{Ma}$ an. Diese werden von zwei Datenpunkten bei 800 Ma gefolgt, bevor eine Lücke bis 950 Ma vorliegt. Zehn Datierungen bilden Grenvillian Alter ab und liegen zwischen 950 und 1130 Ma. Zwei Alter liegen außerhalb des beschrieben Spektrums und ergeben 1830 und 1940 Ma. Die Th/U Verhältnisse der Daten über $600 \mathrm{Ma}$ liegen bis auf eine Ausnahme alle zwischen 0.19 und 1.97, was auf ein magmatisches Wachstum hindeutet. Da es sich um einen Orthogneis handelt, liegen im Gegensatz zu den anderen Proben keine detritischen Zirkone vor. Trotzdem fällt auf, dass bis auf die Zirkone unter 520 Ma die gleiche Altersverteilung wie bei A73-05 aus dem Conlara Metamorphic Complex und den Proben aus den Sierras de Chepes vorliegt. 


\section{Diskussion}

Als Folge von Verwitterung im Liefergebiet und beim Transport von Sedimenten zu ihrem Ablagerungsraum erfolgt eine relative Verarmung von Alkali- und Erdalkalielementen und eine Anreicherung von $\mathrm{Al}_{2} \mathrm{O}_{3}$ (Nesbit et al. 1980; Grandstaff et al. 1986; Harnois 1988). Der Mittelwert des CIA aller als Tonstein (Herron 1988) klassifizierter Proben liegt bei 69. Typische Tonsteine, bzw. deren metamorphe Produkte haben CIA Werte zwischen 70 und 75. Die übrigen analysierten Gesteine besitzen einen Mittelwert von 62, leicht über dem Wert typischer Granite, der um 50 liegt (Nesbit und Young 1982). Es liegt also keine intensive, sondern lediglich eine moderate Verwitterung vor. Bei einem Blick auf das CIA Diagramm (Abb. 5) ist eine Abweichung vom idealen Verwitterungstrend sichtbar, wenn alle dargestellten Punkte betrachtet werden und die Charakteristika der einzelnen Gesteine nicht berücksichtigt werden. Werden diese Charakteristika mit einbezogen und wird mit einberechnte, dass unterschiedliche Ausgangsprodukte (Granit, Tonalit) an verschiedenen Stellen des Diagramms beginnen, so kann zumindest nicht von einer deutlichen Abweichung vom idealen Verwitterungstrend durch z.B. eine $\mathrm{K}_{2} \mathrm{O}$ Metasomatose gesprochen werden. Die Verwitterung besteht hauptsächlich im Abbau von Plagioklas und Kalifeldspat. Im Gegensatz zu früheren geochemischen Arbeiten in der Sierra de San Luis und der Puncoviscana Formation (Sims et al. 1998; Brogioni et al. 2001; López de Luchi et al. 2003; Zimmermann 2005) ist nur ein leichter Trend zur A-K Achse des CIA Diagramms (Abb. 5) zu erkennen. Lediglich bei den Gesteinen der Sierras de Córdoba könnte eine etwas stärkere Tendenz zur A-K Achse zu erkennen sein. Bei den Datenpunkten aus dem linken unteren Bereich des Diagramms handelt es sich bei fünf von sechs Proben um mittelgradig metamorphe Gneise. Die hochgradig metamorphen Gesteine sind tendenziell zu höheren $\mathrm{K} / \mathrm{CN}$ Verhältnissen verschoben, auch wenn sich ihre chemische Zusammensetzung in den übrigen Punkten ähnelt. Einzige Ausnahme bildet A102-06 aus der Sierra Norte, die mit ihrer Hauptelementchemie und einem resultierenden CIA von 36 eher einem basischen Vulkanit oder Plutonit entspricht. Da in diesem Gebiet lediglich eine geringe Aufarbeitung der Gesteine festzustellen war (Abb. 8), könnte die kürzere Distanz zur A-K Achse allerdings auch mit einer höheren $\mathrm{K}_{2} \mathrm{O}$ Konzentration aufgrund einer höheren Glimmerkonzentration im Liefergebiet erklärt werden, der noch nicht verwittert ist. Ausgehend davon, dass keine wesentliche Variation der Hauptelementchemie vorliegt, hatten die Gesteine des Conlara Metamorphic Complex, der Puncoviscana Formation und der Sierras de Córdoba ein Liefergebiet, das aus granodioritischem Material aufgebaut wurde, die (Meta)Sedimente der Sierras de Chepes und 
die Sierra Norte aus einem granodioritischen bis granitischen Liefergebiet stammen und die San Luis Formation aus einem granitischen Ausgangsgestein beliefert wurde. Der Pringles Metamorphic Complex scheint von zwei Liefergebieten versorgt worden zu sein, zum einen aus einem tonalitischen Liefergebiet und zum anderen aus einem granitischen. Eine Aussage zum Nogolí Metamorphic Complex ist schwieriger, da aus diesem Gebiet nur vier Proben vorliegen. Entweder liegen auch hier zwei Liefergebiete vor oder die Proben A39-01 und A16-06 wurden durch eine Kalium Metasomatose beeinflusst.

\subsection{Haupt- und Spurenelemente}

Bei Betrachtung der Th/Sc Verhältnisse fällt auf, dass der größte Teil der Proben über dem von McLennan et al. (1990) für die kontinentale Kruste angegebenen Wert von 0.8 liegt. Die einzige Ausnahme bilden hierbei die Sierras de Córdoba, von der sieben von 16 Proben unterhalb dieses Grenzwertes liegen (Abb. 7). Dies wurde vorher lediglich für Proben des Conlara Metamorphic Complex und - etwas weniger stark ausgeprägt - auch in der San Luis Formation beobachtet, kann aber bei Betrachtung der Daten dieser Arbeit nicht bestätigt werden (Sims et al. 1997; Brogioni et al. 2001; López de Luchi et al. 2003). Im Gegensatz dazu sind die Th/Sc Verhältnisse des Pringles Metamorphic Complex im Durchschnitt deutlich höher als in allen anderen betrachteten Einheiten. Diese Daten schließen einen hohen vulkanischen Eintrag in die östlichen Sierras Pampeanas und die Cordillera Oriental aus. Lediglich ein Teil der Proben aus den Sierras de Chepes deutet auf einen stärkeren vulkanischen Einfluss hin. Dies ist sonst nur bei einzelnen Proben aus den jeweiligen Einheiten zu beobachten (A37-06, A81-06). Im Gegensatz dazu scheinen die Proben aus dem Pringles Metamorphic Complex am stärksten aufbereitet worden $\mathrm{zu}$ sein. Es ist kein mafischer oder vulkanischer Eintrag nachzuweisen, ähnlich dem Nogolí Metamorphic Complex, auch wenn dieser etwas geringere $\mathrm{Th} / \mathrm{Sc}$ Werte aufweist.

Wird das Th/Sc Verhältnis gegen das $\mathrm{Zr} / \mathrm{Sc}$ Verhältnis aufgetragen (Abb. 8), so kann zusätzlich zur Entwicklung des mafischen bzw. sauren Eintrags in ein Sedimentbecken eine Abschätzung des Recycling getroffen werden. Dies ist möglich, da sich $\mathrm{Zr}$ bei fortwährender Aufarbeitung eines Sediments anreichert und somit das $\mathrm{Zr} / \mathrm{Sc}$ Verhältnis steigen sollte, je reifer ein Sediment ist. Es ist nur bei zwei Proben des Pringles Metamorphic Complex (A1306 und A19-06) eine deutliche Recyclingtendenz festzustellen. Alle anderen Proben weisen lediglich einen geringen Recyclingbetrag auf. Trotzdem sind in den verschiedenen Einheiten Unterschiede auszumachen. Die Th/Sc Verhältnisse des Pringles Metamorphic Complex sind 
bei vergleichbaren $\mathrm{Zr} / \mathrm{Sc}$ Verhältnissen höher als bei fast allen anderen untersuchten Proben. Dies deutet auf eine stärker granitisch geprägte und/oder auf stärker aufgearbeitete Liefergebiete hin. Der Conlara Metamorphic Complex zeigt vergleichbare Th/Sc Werte, wie die Puncoviscana Formation. Obwohl die $\mathrm{Zr} / \mathrm{Sc}$ Verhältnisse beider Einheiten nicht hoch sind, so fällt dennoch auf, dass die $\mathrm{Zr} / \mathrm{Sc}$ Verhältnisse des Conlara Metamorphic Complex höher sind als in der Puncoviscana Formation. Ähnlich den reinen Th/Sc Daten (Abb. 7) fällt auch im Th/Sc vs. Zr/Sc Diagramm (Abb. 8) auf, dass ein Teil der Proben aus den Sierras de Córdoba fast keine $\mathrm{Zr}$ Akkumulation zeigen und somit auch fast unaufbereitet abgelagert wurden. Die Proben der Sierras de Córdoba können allerdings in verschiedene Gruppen unterteilt werden. Die hochgradig metamorphen Gesteine deuten auf ein geringeres Recycling hin als die schwach- bis mittelgradig metamorphen Proben. Es besteht also die Möglichkeit, dass aufgrund des hohen Metamorphosegrades und der damit verbundenen Position der Gesteine in der unteren Kruste diese Proben einem Einfluss des oberen Erdmantels ausgesetzt waren, der sich in erhöhten Sc bzw. geringen Zr Werten widerspiegelt. Die Proben der Sierras de Chepes und der Sierra Norte zeigen ebenfalls nur einen sehr geringen Recyclingtrend an, haben aber höhere Durchschnittswerte von $\mathrm{Zr} / \mathrm{Sc}$ als die Proben der Sierras de Córdoba.

Obwohl die Proben aus den Sierras de Córdoba einen stärkeren Manteleinfluss aufweisen, entspricht ihre negative Eu/Eu* Anomalie der typischen kontinentalen Oberkruste (McLennan et al. 1990). Im Unterschied zu den vorher betrachteten $\mathrm{Th} / \mathrm{Sc}$ und $\mathrm{Zr} / \mathrm{Sc}$ Werten ist bei den $\mathrm{Eu} / \mathrm{Eu}^{*}$ Anomalien kein nennenswerter Unterschied zwischen den jeweiligen Einheiten zu erkennen, da alle Einheiten negative Anomalien mit Werten zwischen 0.5 und 0.6 aufweisen (Abb. 9).

Wenn Diagramme zur Bestimmung der geotektonischen Position oder zur Bestimmung des Liefergebietes herangezogen werden, kann generell gesagt werden, dass ein Großteil der Proben in das Feld eines kontinentalen Inselbogens fällt (Abb. 6, 10 - 13). Es fallen erneut die Proben auf, welche bereits bei den $\mathrm{Th} / \mathrm{Sc}$ und $\mathrm{Zr} / \mathrm{Sc}$ Diagrammen hohe, auf Recycling hindeutende Werte zeigten und hier in den Bereich eines passiven Kontinentalrandes fallen (A13-06 und A19-06). Die Datenpunkte der Sierras de Córdoba liegen auch hier näher am Feld für ozeanischen Inselbogen als die anderen Einheiten. In den meisten Fällen liegen die Datenpunkte dennoch klar innerhalb eines kontinentalen Inselbogens, bzw. zeigen einen stärker entwickelten Inselbogen mit saureren Gesteinen als Liefergebiet an. Auch wenn es besonders in den Sierras de Córdoba - Tendenzen zu einem vermehrten Eintrag vulkanischen Materials gibt, ist der Unterschied bezüglich des geotektonischen Milieus der verschiedenen Einheiten zueinander nicht erheblich. Trotzdem scheint der vulkanische Einfluss in den 
Sierras de Córdoba größer als in allen anderen untersuchten Gebieten der östlichen Sierras Pampeanas zu sein und kann nur mit Daten des Conlara Metamorphic Complex (López de Luchi et al. 2003) verglichen werden. Dies gilt nicht nur für das Geotektonische Milieu, sondern auch für die $\mathrm{Th} / \mathrm{Sc}$ und $\mathrm{Zr} / \mathrm{Sc}$ Werte. Auch wenn die Provenienz der Metasedimente der Sierras de Córdoba deutlich basischer ist als die der übrigen, so fallen doch zwei Proben auf (A68-06 und A70-06), die auf einen passiven Kontinentalrand hindeuten (Abb. 12, 13). Aufgrund ihres hohen $\mathrm{TiO}_{2}$ Gehalts liegen sie außerhalb jeglicher geotektonischer Position bei den Hauptelementen (Abb. 6). Der hohe $\mathrm{TiO}_{2}$ Gehalt könnte auf eine große Konzentration von Tonmineralen hindeuten. Diese Vermutung wird auch durch die REE Konzentrationen von über 300 ppm gestützt. In allen übrigen Diagrammen zeigen diese beiden Proben die stärksten Tendenzen zu einem passiven Kontinentalrand in den Sierras de Córdoba. Dies gilt, zusätzlich zu den Proben A13-06 und A19-06 aus dem Pringles Metamorphic Complex, in ähnlicher Form für die stark $\mathrm{SiO}_{2}$-haltige Probe A12-06 aus der San Luis Formation, A33-06 aus den Sierras de Chepes und A87-06 aus der Puncoviscana Formation. Es liegt aus fast jeder Einheit eine Probe vor, die auf einen passiven Kontinentalrand, zumindest aber auf erhöhtes Recycling hindeutet. Das Liefergebiet scheint also ähnlich, wenngleich auch nicht absolut homogen gewesen zu sein. Zumindest ist dies anhand geochemischer Daten nicht eindeutig zu klären. Die Datenpunkte legen nahe, dass es sich bei dem geotektonischen Milieu um einen kontinentalen Inselbogen handelt. Dies lässt die Sedimentation in ein ForeArc und ein Back-Arc zu. López de Luchi et al. (2003) argumentierten aufgrund hoher Th/U Werte gegen ein Fore-Arc als geotektonischen Ort - zumindest für die Metasedimente der Sierra de San Luis. Zimmermann (2005) hingegen favorisiert ein solches Fore-Arc als geotektonische Milieu und argumentierte, dass die hohen $\mathrm{Th} / \mathrm{U}$ Werte eine Folge starker Verwitterung seien, die bei seinen Daten anhand einer positiven Ce Anomalie sichtbar sei. Da in den vorliegenden Daten eine solche Ce Anomalie nicht vorliegt, sollte der Einfluss der Verwitterung auf die Spurenelemente eher gering sein. Aus diesem Grund müssten die erhöhten Th/U Werte auf ein Back-Arc Becken als Sedimentationsraum hindeuten, auf das im Falle der Sierras de Córdoba ein stärkerer mafischer/vulkanischer Einfluss ausgeübt wurde als in den übrigen Einheiten.

\subsection{Schlussfolgerungen aus den Sm-Nd WR Daten}

Die nach Goldstein et al. (1984) neu berechneten Literaturwerte der Sm-Nd Daten ergaben $\mathrm{T}_{\mathrm{DM}}$ Modellalter zwischen $1.8-1.5 \mathrm{Ga}$ und $\varepsilon \mathrm{Nd}_{(540 \mathrm{Ma})}$ Werte zwischen -3.9 und -6.3 für die 
metasedimentären Einheiten der östlichen Sierras Pampeanas (u.a. Rapela et al. 1998; Bock et al. 2000; Steenken et al. 2004; Escayola et al. 2007). Die Achavil und Negro Peinando Formation (Abb. 1) haben etwas höhere $\mathrm{T}_{\mathrm{DM}}$ Modellalter zwischen 2.0 und $1.8 \mathrm{Ga}$ und etwas stärker entwickelte $\varepsilon \mathrm{Nd}_{(540 \mathrm{Ma})}$ Werte von -6.6 bis -9.4. Die $\mathrm{T}_{\mathrm{DM}}$ Modellalter des Nogolí Metamorphic Complex zwischen 1.70 und 1.64 Ga und die $\varepsilon \mathrm{Nd}_{(540)}$ Daten zwischen -5.3 und -5.6 sind kaum von denen des Conlara Metamorphic Complex und des Pringles Metamorphic Complex zu unterscheiden und liegen im Bereich der von Steenken et al. (2004) gemessenen Werte (1.8 - $1.5 \mathrm{Ga})$. In den Nd-Daten des Pringles Metamorphic Complex können einige Auffälligkeiten, die bereits in der Geochemie angesprochen wurden, ebenfalls wieder gefunden werden. Die Proben, die keine Anzeichen eines erhöhten Recyclings aufwiesen (A14-06 und A15-06), haben geringere $\mathrm{T}_{\mathrm{DM}}$ Modellalter von ca. $1.65 \mathrm{Ga}$ und $\varepsilon \mathrm{Nd}_{(540)}$ Verhältnisse von -5.3 und -5.9. Die zumindest in einigen Diagrammen (Abb. 12 und 13) in die Nähe eines passiven Kontinentalrandes verschobene psammopelitische Probe A18-06 weist ein etwas höheres $\mathrm{T}_{\mathrm{DM}}$ Modellalter von $1.74 \mathrm{Ga}$ und einen $\varepsilon \mathrm{Nd}_{(540)}$ Wert von -6.0 auf und eine der am deutlichsten zu einem passiven Kontinentalrand tendierende Probe (A13-06) hat mit $1.83 \mathrm{Ga}$ das höchste $\mathrm{T}_{\mathrm{DM}}$ Modellalter und das niedrigste $\varepsilon \mathrm{Nd}_{(540)}$ Verhältnis von -6.7. In dieser Kollektion (Anhang 2) sind derartige Werte wie für A13-06 in der Sierra de San Luis sonst nur in der San Luis Formation anzutreffen. Die Daten aus dem Conlara Metamorphic Complex zeigen nur eine geringe Schwankung an. Drei der vier Proben haben eine homogene Verteilung der $\varepsilon \mathrm{Nd}_{(540)}$ Daten von -4.8 bis -5.3 und $\mathrm{T}_{\mathrm{DM}}$ Modellalter zwischen 1.69 und 1.65 Ga. A61-05 hat ein geringeres $\mathrm{T}_{\mathrm{DM}}$ Modellalter von 1.54 Ga und auch der $\varepsilon \mathrm{Nd}_{(540)}$ Wert ist mit -3.0 erhöht, obwohl die Probe geochemisch keinerlei Auffälligkeiten zeigt. Dies steht im Widerspruch zu den von Steenken et al. (2004) erhaltenen Werten $\left(\mathrm{T}_{\mathrm{DM}}\right.$ 1.83 - $1.80 \mathrm{Ga} ; \varepsilon \mathrm{Nd}_{(540)}-6.3$ bis -8$)$. Anhand dieser Daten scheint der Conlara Metamorphic Complex nicht so homogen zu sein wie früher angenommen. Die $\mathrm{T}_{\mathrm{DM}}$ Modellalter zwischen 1.80 Ga und $1.63 \mathrm{Ga}$ und die $\varepsilon \mathrm{Nd}_{(540)}$ Werte von -3.9 bis -6.3 in der Puncoviscana Formation stimmen mit den Daten von Bock et al. (2000) überein ( $\mathrm{T}_{\mathrm{DM}}$ Modellalter zwischen 1.8 und 1.6 $\mathrm{Ga}$ und $\varepsilon \mathrm{Nd}_{(540)}$ von -5.7 bis -6.4$)$. Die niedrigsten Werte liegen bei zwei niedriggradig metamorphen, pelitischen Gesteinen vor (A85-06 und A86-06), die ebenso die geringsten Th/Sc Verhältnisse von 0.75 und 0.73 der Puncoviscana Formation aufweisen. Dies könnte auf Eintrag juvenilen Materials in diese Proben hindeuten, der die $\mathrm{T}_{\mathrm{DM}}$ Modellalter verringert und die $\varepsilon \mathrm{Nd}_{(540)}$ Werte erhöht hat. Werden diese beiden Proben von der Betrachtung ausgeschlossen, so ergibt sich eine fast komplette Überschneidung der Nd-Daten der Puncoviscana Formation und des Arequipa-Antofalla Kratons, was dies zu einem möglichen 
Liefergebiet macht. Die Daten der Sierras de Córdoba beinhalten eine Gruppe von sechs Proben, die geringe $\mathrm{T}_{\mathrm{DM}}$ Modellalter von $1.63-1.57 \mathrm{Ga}$ zusammen mit relativ hohen $\varepsilon \mathrm{Nd}_{(540)}$ Werten zwischen -3.2 und -4.1 aufweisen. Diese Werte stehen im Kontrast zu den hohen $\mathrm{T}_{\mathrm{DM}}$ Modellaltern von $1.88 \mathrm{Ga}$ und niedrigen der $\varepsilon \mathrm{Nd}_{(540)}$ Verhältnissen von -5.2 und -6.2 der beiden granitischen Diatexite A61-06 und A80-06. Solch inhomogene Daten wurden ebenso von Rapela et al. (1998) für die Sierras de Córdoba publiziert und können, wie oben erwähnt, auch in der Sierra de San Luis beobachtet werden, wenn die Datensätze dieser Arbeit mit vorangegangenen kombiniert werden. Ein Zusammenhang der geochemischen Daten mit den $\mathrm{T}_{\mathrm{DM}}$ Altern kann in den Sierras de Córdoba im Gegensatz zum Pringles Metamorphic Complex nicht gemacht werden. Die beiden Proben (A68-06 und A70-06), die die stärkste Tendenz zu einem passiven Kontinentalrand zeigen, haben beide geringe $\mathrm{T}_{\mathrm{DM}}$ Modellalter von 1.6 Ga. Das Alter von 1.88 Ga von Probe A80-06 kann möglicherweise mit dem hohen Th/Sc Verhältnis von 2.7 und der ausgeprägten negativen Eu/Eu* Anomalie von 0.42 erklärt werden. Beide deuten auf eine erhöhte Reife des Ausgangsmaterials hin. Im Fall von A61-06 ist eine solche Argumentation allerdings nicht möglich, da hier ein Th/Sc Verhältnis von 0.6 und eine negative Eu/Eu* Anomalie von 0.61 vorliegt. Beide Proben haben die höchsten ${ }^{147} \mathrm{Sm} /{ }^{144} \mathrm{Nd}$ Verhältnisse von 0.133 und 0.127 der gesamten Datenkollektion, sowie ein geringes $\mathrm{La}_{\mathrm{N}} / \mathrm{Sm}_{\mathrm{N}}$ Verhältnis von 2.6 und 2.9, was auf eine Mobilisierung der LREE hinweisen kann (z.B. Frei et al. 1999). Somit könnte das Sm-Nd Verhältnis dieser Proben gestört sein und die Werte verfälschen. Die Sm-Nd Daten der Sierra Norte sind im Vergleich zu denen der direkt südlich angrenzenden Sierras de Córdoba stärker entwickelt. Die $\varepsilon \mathrm{Nd}_{(540)}$ Werte liegen zwischen -6.0 und -7.6 und die $\mathrm{T}_{\mathrm{DM}}$ Modellalter variieren zwischen 1.85 und $1.72 \mathrm{Ga}$. Ein deutlicher Unterschied zu den zuvor diskutierten Einheiten ist das Fehlen von $\mathrm{T}_{\mathrm{DM}}$ Modellaltern unter $1.7 \mathrm{Ga}$ und $\varepsilon \mathrm{Nd}_{(540)}$ Verhältnissen über -6 (Rapela et al. 1998; Bock et al. 2000; Steenken et al. 2004; Escayola et al. 2007). All diese Autoren haben inhomogene $\mathrm{T}_{\mathrm{DM}}$ Modellalter zwischen 1.9 und 1.5 Ga erhalten. Die Daten der San Luis Formation und der Sierras de Chepes sind mit denen der Sierra Norte vergleichbar. Vier der fünf untersuchten Proben der niedriggradig metamorphen Pelite der San Luis Formation haben $\varepsilon \mathrm{Nd}_{(540)}$ Daten zwischen -6.6 und -7.8. Steenken et al. (2004) gaben sogar noch etwas stärker entwickelte Werte von -7.4 bis -7.9 an. Bei den $\mathrm{T}_{\mathrm{DM}}$ Modellaltern liegen vier der fünf Proben zwischen 1.86 und $1.74 \mathrm{Ga}$, ähnlich den Werten von Steenken et al. (2004), die Daten zwischen 1.82 und $1.72 \mathrm{Ga}$ erhielten. Die Proben aus den Sierras de Chepes liegen mit $\mathrm{T}_{\mathrm{DM}}$ Altern von 1.89 - 1.77 Ga und $\varepsilon \mathrm{Nd}_{(540)}$ Verhältnisse von -6.6 bis -7.7 in einem ähnlichen Bereich wie die San Luis Formation und die Daten von Pankhurst et al. (1998), die bei zwei Proben aus den 
Sierras de Chepes $\mathrm{T}_{\mathrm{DM}}$ Modellalter von 1.79 und $1.78 \mathrm{Ga}$ und $\varepsilon \mathrm{Nd}_{(540)}$ Werte von -7.7 und -6.6 erhielten. Die ist mit den Los Túneles Phylliten aus den Sierras de Córdoba mit einem $\varepsilon \mathrm{Nd}_{(540)}$ Verhältnis von -6.6 und einem $\mathrm{T}_{\mathrm{DM}}$ Modellalter von $1.80 \mathrm{Ga}$ vergleichbar (Rapela et al. 1998). Interessanterweise hat die Probe mit dem höchsten $T_{\mathrm{DM}}$ Modellalter von 1.89 (A3706) der Sierras de Chepes das geringste Th/Sc Verhältnis von 0.6. Ähnlich den beiden Proben der Sierras de Córdoba (A61-06 und A80-06) mit dem mit Abstand höchsten Modellalter ist das $\mathrm{La}_{\mathrm{N}} / \mathrm{Sm}_{\mathrm{N}}$ Verhältnis dieser Probe mit 2.8 deutlich geringer als das der übrigen Proben dieser Einheit (3.4). Dies könnte auf eine Mobilisierung der LREE (z.B. Frei et al. 1999) und damit auf eine Störung des Sm-Nd Isotopensystems hindeuten. Wenn diese Probe nicht berücksichtigt wird, ergeben sich $\mathrm{T}_{\mathrm{DM}}$ Modellalter von $1.82-1.77 \mathrm{Ga}$ und $\varepsilon_{\mathrm{Nd}}(540)$ Verhältnisse von -6.6 bis -7.2. Vergleichbare und teilweise sogar höhere $\mathrm{T}_{\mathrm{DM}}$ Modellalter und geringere $\varepsilon \mathrm{Nd}_{(540)}$ Verhältnisse wurden nur von Collo et al. (2009) aus der Negro Peinado Formation $\left(\mathrm{T}_{\mathrm{DM}} 1.96-1.74 \mathrm{Ga}\right.$ und $\varepsilon \mathrm{Nd}_{(540)}-6.6$ bis -8.2) und der Achavil Formation $\left(\mathrm{T}_{\mathrm{DM}}\right.$ $1.99-1.78 \mathrm{Ga}$ und $\varepsilon \mathrm{Nd}_{(540)}-8.2$ bis -9.4$)$ aus dem Famatina Belt berichtet.

Als geodynamisches Szenario haben Escayola et al. (2007) einen magmatischen Bogen mit nach Westen gerichteter Subduktion im östlichen Bereich der Sierras de Córdoba zwischen 760 und $600 \mathrm{Ma}$ vorgeschlagen. Diese Subduktion ging mit der Annäherung des Pampia Terranes einher, welches von Altern der Grenvillian Orogenese dominiert war. Diese Autoren beschreiben ein Ost-West Profil durch die Sierras de Córdoba mit einer kontinuierlichen Abnahme der $\mathrm{T}_{\mathrm{DM}}$ Modellalter nach Osten aufgrund eines zunehmenden vulkanischen Einflusses in Richtung des magmatischen Bogens, was die Verringerung der $\mathrm{T}_{\mathrm{DM}}$ Modellalter erklärt. Eine solche Beobachtung kann anhand der in dieser Arbeit vorgelegten Daten nicht bekräftigt werden. Es liegt keine Alterstendenz in eine bestimmte Richtung oder zu einem bestimmten Metamorphosegrad vor. Auch wenn es anhand der neuen Daten schwierig ist, ein Profil nach Osten abnehmender $\mathrm{T}_{\mathrm{DM}}$ Modellalter in den Sierras de Córdoba festzulegen, so liegt doch eine Tendenz zu jüngeren $(<1.7 \mathrm{Ga}) \mathrm{T}_{\mathrm{DM}}$ Modellaltern im östlichen Bereich der östlichen Sierras Pampeanas wie den Sierras de Córdoba, dem Conlara Metamorphic Complex und der Puncoviscana Formation, die bereits von Steenken et al. (2006) in eine Beziehung gesetzt wurden und einigen Einheiten im westlichen Gebiet, wie der San Luis Formation, den Sierras de Chepes, der Negro Peinado und der Achavil Formation (Collo et al. 2009) vor. Die Sedimentation der letzteren Einheiten fand nach der Ablagerung der Sierras de Córdoba, des Conlara Metamorphic Complex und der Puncoviscana Formation statt. Daher könnten die höheren $\mathrm{T}_{\mathrm{DM}}$ Modellalter im Westen neben der größeren Entfernung auch mit einer zeitlich bedingten Abnahme der vulkanischen Aktivität des magmatischen Bogens in 
Verbindung gebracht werden. Die $\mathrm{T}_{\mathrm{DM}}$ Modellalter der Mesón Group sind mit denen der Sierras de Chepes, der San Luis Formation und denen des Famatina Belts vergleichbar (Steenken et al. 2004; Collo et al. 2009), allerdings liegen aus dieser Formation nur vier Datenpunkte vor, von denen drei zwischen 1.82 - 1.74 Ga liegen und ein deutlich geringeres $\mathrm{T}_{\mathrm{DM}}$ Modellalter von $1.46 \mathrm{Ga}$ aufweist (Bock et al. 2000). Abgesehen von diesem jungen $\mathrm{T}_{\mathrm{DM}}$ Modellalter können die Sm-Nd-Daten auch mit den geochemischen Daten in Verbindung gebracht werden, die einen höheren vulkanischen/mafischen Eintrag in den Sierras de Córdoba und teilweise auch in den Conlara Metamorphic Complex (López de Luchi et al. 2003) anzeigen. In diesem Modell wäre allerdings die Sierra Norte problematisch, die vergleichbare $\mathrm{T}_{\mathrm{DM}}$ Modellalter wie die erwähnten westlichen Einheiten besitzt, allerdings unmittelbar nördlich der Sierras de Córdoba liegt.

Trotz des direkten Kontakts der östlichen Sierras Pampeanas mit dem Río de la Plata Kraton (Rapela et al. 2007) wurden keine für den Río de la Plata Kraton typischen $\mathrm{T}_{\mathrm{DM}}$ Modellalter zwischen 2.7 - 2.2 Ga (Cingolani et al. 2002; Hartmann et al. 2002; Pankhurst et al. 2003; Rapela et al. 2007) in den östlichen Sierras Pampeanas gefunden. Dies schließt den Río de la Plata Kraton als Hauptquelle der (Meta)Sedimente aus. Rapela et al. (2007) stellten die These auf, dass der Arequipa-Antofalla Kraton Teil eines großen kontinentalen Gebietes war, das ebenso die westlichen Sierras Pampeanas und den Amazonia Kraton umfasste. Dieser Kontinent wäre nach diesem Modell mit dem Río de la Plata Kraton und dem Kalahari Kraton kollidiert, wodurch die pampeane Orogenese ausgelöst wurde, die für den größten Teil der metamorphen Strukturen im Conlara Metamorphic Complex, den Sierras de Córdoba und der Sierra Norte verantwortlich ist (Rapela et al. 2007; Steenken et al. 2007; Siegesmund et al. 2009). Rapela et al. (2007) führten die Saldania Group aus Südafrika als mögliches Äquivalent der Puncoviscana Formation an. Diese Einheit hat Sm-Nd Modellalter zwischen 1.6 und $1.5 \mathrm{Ga}$ (da Silva et al. 2000) und ist damit nur geringfügig jünger als die Daten aus den östlichen Sierras Pampeanas. Die $\mathrm{T}_{\mathrm{DM}}$ Alter des Arequipa-Antofalla Kratons liegen mit Ausnahme eines Alters von 1.3 Ga zwischen 1.9 - 1.5 Ga (Lucassen et al. 2000), was auch diese Einheit zu einem potentiellen Liefergebiet macht.

Generell können mögliche Liefergebiete der Metasedimente der östlichen Sierras Pampeanas entweder im Norden (Südbrasilien, Chile, Bolivien, Peru) oder im südlichen Afrika gelegen haben. Da der Río de la Plata Kraton aus oben genannten Gründen als Liefergebiet der Östlichen Sierras Pampeanas ausscheidet, müssen die Protolithe in einem Nord-Süd streichenden Becken abgelagert worden sein. Die Sedimente, die dieses Becken füllten, wurden dann entweder von Norden, Süden oder aus beiden Richtungen geschüttet. Eine 
weitere Möglichkeit ist, dass Gesteine mit einer ähnlichen $\mathrm{Nd}$ Isotopie und ähnlicher Geochemie wie die der östlichen Sierras Pampeanas vorliegenden Metasedimente auf dem Río de la Plata Kraton gelegen haben, heute aber komplett erodiert sind. Ist dies nicht der Fall, dann müssen die östlichen Sierras Pampeanas entlang von Scherzonen an ihre heutige Position direkt neben dem Río de la Plata Kraton gebracht worden sein wie es z.B. auch bei Rapela et al. (2007) und Siegesmund et al. (2009) diskutiert wird.

\subsection{Schlussfolgerungen aus den WR Pb-Pb Daten}

Die Entwicklung des ${ }^{207} \mathrm{~Pb}$, welches aus ${ }^{235} \mathrm{U}$ entsteht, ist in den verschiedenen untersuchten Gebieten praktisch nicht zu unterscheiden. Lediglich die Arkose A12-06 aus der San Luis Formation und eine psammitische Probe aus der Puncoviscana Formation (A88-06) haben ein erhöhtes ${ }^{207} \mathrm{~Pb} /{ }^{204} \mathrm{~Pb}$ Verhältnis (Abb. 16). Werden Vergleichswerte von Bock et al. (2000) und Schwartz und Gromet (2004) herangezogen, so ist keine Abweichung der hier vorgestellten Werte zu den Literaturwerten erkennbar. Die ${ }^{207} \mathrm{~Pb} /{ }^{204} \mathrm{~Pb}$ Verhältnisse sind mit denen der granitischen Gneise des Sunsas Orogens in Südwest Brasilien (Geraldes et al. 2001) und dem Arequipa-Antofalla Kraton (Loewy et al. 2003, 2004) vergleichbar. Diese Autoren verknüpften auch die Pb-Isotopien des nördlichen Bereichs des Arequipa-Antofalla Kratons mit der Richtersveld Domain (Kalahari Kraton) und den zentralen Bereich des ArequipaAntofalla Kratons mit dem Natal Belt (Kalahari Kraton). Solch eine Unterscheidung ist anhand der hier vorgestellten Daten nicht möglich, da sich die Datenpunkte in den oben erwähnten Gebiete überlappen (Abb. 16). Im Diagramm der ${ }^{208} \mathrm{~Pb} /{ }^{204} \mathrm{~Pb}$ vs. ${ }^{206} \mathrm{~Pb} /{ }^{204} \mathrm{~Pb}$ Daten (Abb. 17) liegt eine stärkere Verteilung der Werte vor, die von der linearen Entwicklung des vorherigen Diagramms abweicht. Die Werte liegen zwar erneut im Überschneidungsbereich des nördlichen Bereichs und des zentralen Bereichs (Belén) des Arequipa-Antofalla Basement und der Richtersveld Domain auf der einen Seite und des südlichen Bereichs sowie des zentralen Bereichs (Choja) des Arequipa-Antofalla Basement und des Natal Belt auf der anderen Seite, allerdings zeigen einige Proben des Pringles Metamorphic Complex und einige der Sierras de Córdoba erhöhte ${ }^{208} \mathrm{~Pb} /{ }^{204} \mathrm{~Pb}$ Werte an. Im Falle des Pringles Metamorphic Complex wurde bereits anhand der Geochemie eine gesonderte Stellung gegenüber den übrigen metasedimentären Proben diskutiert und auf zwei unterschiedliche Liefergebiete hingewiesen, was auch die leicht abweichenden Pb-Isotopien erklären könnte. Die größere Verteilung der Pb-Daten der Sierras de Córdoba könnte auf einen stärker vulkanischen Ursprung hinweisen oder aber durch den höheren Metamorphosegrad dieser Proben 
hervorgerufen worden sein. Erhöhte Verhältnisse im thoro-uranogenen Diagramm (Abb. 17) sind meist auf die Mobilisierung von Uran zurückzuführen, da Th schlechter zu mobilisieren ist. Dabei führt der Verlust von Uran zu geringeren ${ }^{206} \mathrm{~Pb} /{ }^{204} \mathrm{~Pb}$ Verhältnissen, wodurch die ${ }^{208} \mathrm{~Pb} /{ }^{204} \mathrm{~Pb}$ relativ erhöht werden. Ähnliches wurde auch bei unterkrustalen Xenolithen der südlichen Anden $\left(21-27{ }^{\circ} \mathrm{S}\right)$ beobachtet (Lucassen et al. 2001). Die ${ }^{208} \mathrm{~Pb} /{ }^{204} \mathrm{~Pb}$ vs. ${ }^{206} \mathrm{~Pb} /{ }^{204} \mathrm{~Pb}$ Datenpunkte des nördlichen und zentralen (Belén) Bereichs des ArequipaAntofalla Basements liegen über den hier präsentierten Datenpunkten, die des südlichen Bereichs und des zentralen Bereichs (Choja) stimmen allerdings überein (Loewy et al. 2004). Dies spricht gegen eine Herkunft der Gesteine der östlichen Sierras Pampeanas aus dem nördlichen Bereich des Arequipa-Antofalla Kratons. Wenn dieser als Liefergebiet angenommen wird, so muss es sich um den zentralen Bereich (Choja) oder den südlichen Bereich handeln. Ähnliche Bleiverhältnisse veranlassten allerdings Schwartz und Gromet (2004) dazu, den Kalahari Kraton als Liefergebiet anzunehmen.

Festzuhalten bleibt, dass es schwierig ist, bestimmte Provenienzen der (Meta)Sedimente der östlichen Sierras Pampeanas anhand der Pb-Isotopie zu ermitteln, da die Isotopenverhältnisse zwischen denen verschiedener möglicher Liefergebiete liegen. Die Daten sind denen von Schwartz und Gromet (2004) ähnlich, die den Kalahari Kraton als Liefergebiet für die Sierras de Córdoba favorisieren und ebenso mit denen von Bock et al. (2000) vergleichbar, die auf eine homogene Isotopie der nordwestargentinischen und nordchilenischen Kruste verweisen.

\subsection{Schlussfolgerungen der Zirkon SHRIMP Altersdatierungen}

Liefergebietsanalysen in den östlichen Sierras Pampeanas, die auf U-Pb SHRIMP Altersdatierungen an Zirkonen beruhen, beziehen sich meistens auf das Vorkommen eines Grenvillian (um $1000 \mathrm{Ma}$ und eines Brasiliano Maximums (800 - $600 \mathrm{Ma}$ ). Analysierte Zirkone aus der Puncoviscana Formation und dem Conlara Metamorphic Complex zeigen eben solche Altersverteilungen, zumindest aber einen deutlichen Einfluss von detritischen Zirkonen mit Grenvillian Altern. Diese Verteilungsmuster (Abb. 19 b) können auch in anderen Bereichen der östlichen Sierras Pampeanas beobachtet werden, wie z.B. in den Sierras de Córdoba und der Sierra de Ancasti (Schwartz und Gromet 2004; Steenken et al. 2006; Rapela et al. 2007). Anhand von Sm-Nd-Daten und SHRIMP Datierungen schlugen Steenken et al. $(2004,2006)$ bereits den Conlara Metamorphic Complex als höhergradiges Äquivalent der Puncoviscana Formation vor. Im Nogolí Metamorphic Complex fehlen die für die vorherigen Einheiten typischen Grenvillian Alter fast vollständig (eins von 29 Altern), 

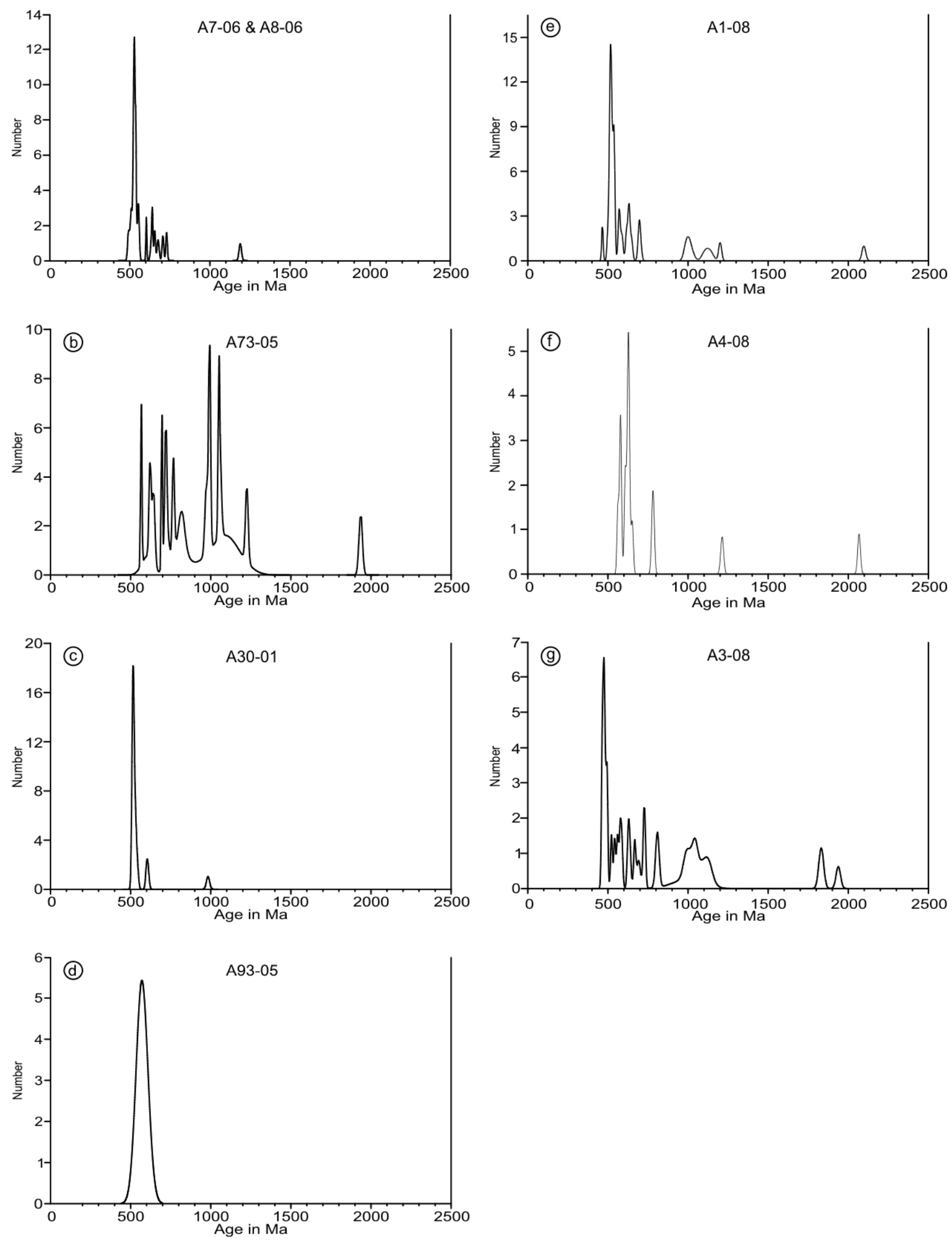

Abbildung 19: Wahrscheinlichkeitsdichte Diagramme der unterschiedlichen Alter der Proben. Aufgrund einer geringen Analysezahl und der bei beiden Proben ähnlichen Altersverteilung werden A7-06 und A8-06 (beide aus dem Nogolí Metamorphic Complex) zusammen in einem Diagramm dargestellt. a) A7-06 und A8-06 aus dem Nogolí Metamorphic Complex, b) A73-05 aus dem Conlara Metamorphic Complex, c) A30-01 aus der San Luis Formation, d) A93-05 aus der San Luis Formation, e) A1-08 aus den Sierras de Chepes, f) A4-08 aus den Sierras de Chepes und g) A3-08 aus dem südlichsten Bereich der Sierras de Córdoba. 
ebenso wie im Pringles Metamorphic Complex, in dem zwei Grenvillian Alter vorliegen, von denen eins jedoch sehr diskordant ist (67\%) und außerdem einen hohen Betrag (3.68 \%) an gewöhnlichem ${ }^{206} \mathrm{~Pb}$ aufweist. Außerdem konnte hier noch ein Zirkonkern mit ca. $2.7 \mathrm{Ga}$ datiert werden (Steenken et al. 2006). Die 26 Datenpunkte eines gebänderten Gneises aus dem Conlara Metamorphic Complex (Abb. 19 b) erstrecken sich über ein Altersspektrum, das sich von pampeanen Altern bei 519 Ma bis zur proterozoisch-neoarchaischnen Grenze um 2500 Ma erstreckt. Die jüngsten Altersdatierungen von 519 und 567 Ma zeigen geringe Th/U Werte (0.03 und 0.11) und stammen von Zirkonrändern. Dies deutet auf ein metamorphes Wachstum hin. Ererbte Zirkonalter beginnen bei $604 \mathrm{Ma}$ und erstrecken sich über die Brasiliano und die Grenvillian Orogenesen. Die beiden ältesten Analysen sind magmatische Alter bei $1860 \mathrm{Ma}$, etwas jünger als die typischen Alter des Río de la Plata Kratons und der Transamazonas Orogenese $(2.0-2.2 \mathrm{Ga})$ und ein Alter um $2700 \mathrm{Ma}$. Entscheidend ist jedoch das Fehlen konkordanter pampeanischer Alter. Eine große Zahl der Brasiliano Alter ist diskordant, sodass die ${ }^{207} \mathrm{~Pb} /{ }^{206} \mathrm{~Pb}$ Alter betrachtet werden sollten. Diese weisen zwar Unterschiede auf, jedoch resultieren immer noch Alter der Brasiliano Orogenese aus den Datenpunkten. Das jüngste verlässliche ${ }^{207} \mathrm{~Pb} /{ }^{206} \mathrm{~Pb}$ Alter liegt bei $581 \pm 52 \mathrm{Ma}$ und sollte das Maximalalter des Conlara Metamorphic Complex anzeigen. Auch wenn dieses einen hohen Fehler aufweist, steht es dennoch im Einklang mit einem metamorphen PbSL Granat Alter von $564 \pm 21 \mathrm{Ma}$ (Siegesmund et al. 2009). Ähnliche Verteilungsmuster von U-Pb Altern wurden auch aus dem nordwestlichen Sektor des Conlara Metamorphic Complex (Steenken et al. 2006) und aus dem nördlichen Bereich der Sierras de Córdoba (Sims et al. 1998; Schwartz und Gromet 2004; Escayola et al. 2007), der Sierra de Ancasti (Rapela et al. 2007) und der Puncoviscana Formation (Adams et al. 2006, 2008) berichtet. Der Großteil der Zirkone stammt aus spätmesoproterozoischen und neoproterozoischen Quellen, was durch Maxima bei $962 \pm 17 \mathrm{Ma}$ und $631 \pm 15 \mathrm{Ma}$ (Rapela et al. 2007) dokumentiert werden kann. Diese Autoren beprobten die regional vorkommenden gebänderten Gneise der Ancasti Formation aus der Sierra de Ancasti. Generell kann von einer bimodalen Verteilung der Alter gesprochen werden, die zum einen zwischen 1100 - $950 \mathrm{Ma}$ und zum anderen zwischen 680 - 570 Ma liegen. Ein untergeordnetes Maximum liegt bei vielen Proben um 1800 Ma. Ältere Datenpunkte im Bereich typischer Alter für den Río de la Plata Kraton zwischen 2.0 und 2.2 Ga kommen nur selten vor. Dies gilt sowohl für den Conlara Metamorphic Complex, die Puncoviscana Formation und die Sierras de Córdoba (Sims et al. 1998; Schwartz and Gromet 2004; Adams et al. 2006, 2008; Steenken et al. 2006; Escayola et al. 2007; Siegesmund et al. 2009). Die Proben des Nogolí Metamorphic Complex werden von pampeanen magmatischen Altern 

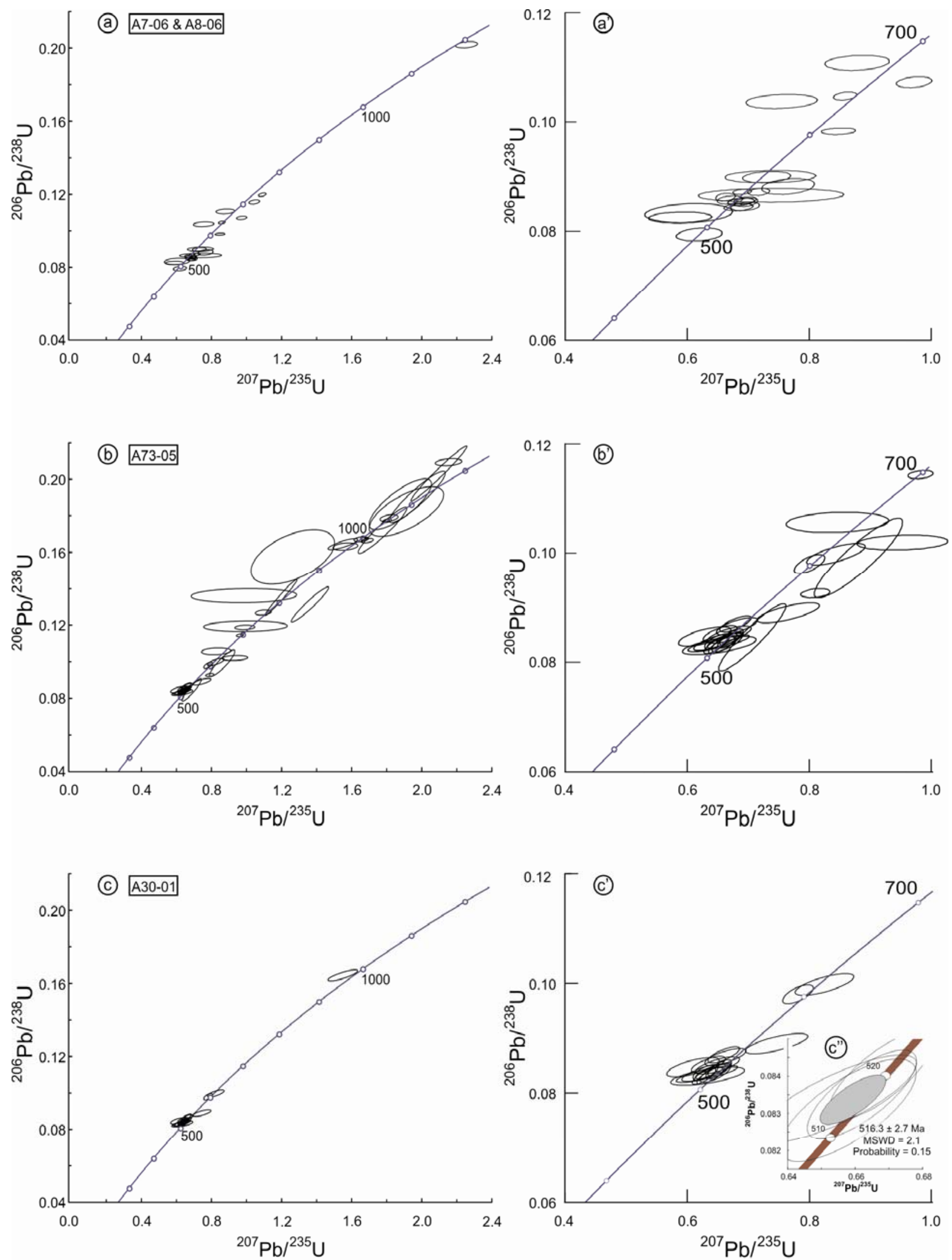

Abbildung 20: Wetherill Concordia Diagramme der Proben (a) A7-06 und A8-06 aus dem Nogolí Metamorphic Complex, (b) A73-05 aus dem Conlara Metamorphic Complex und (c) A30-01 aus der San Luis Formation. Die Abbildungen a' - c' beziehen sich auf die gleichen Proben und sind eine Vergrößerung des Altersbereichs bis 700 Ma. In Abbildung $20 \mathrm{c}$ "' ist die Berechnung eines konkordanten Alters von 516.3 \pm 2.7 Ma dargestellt. 

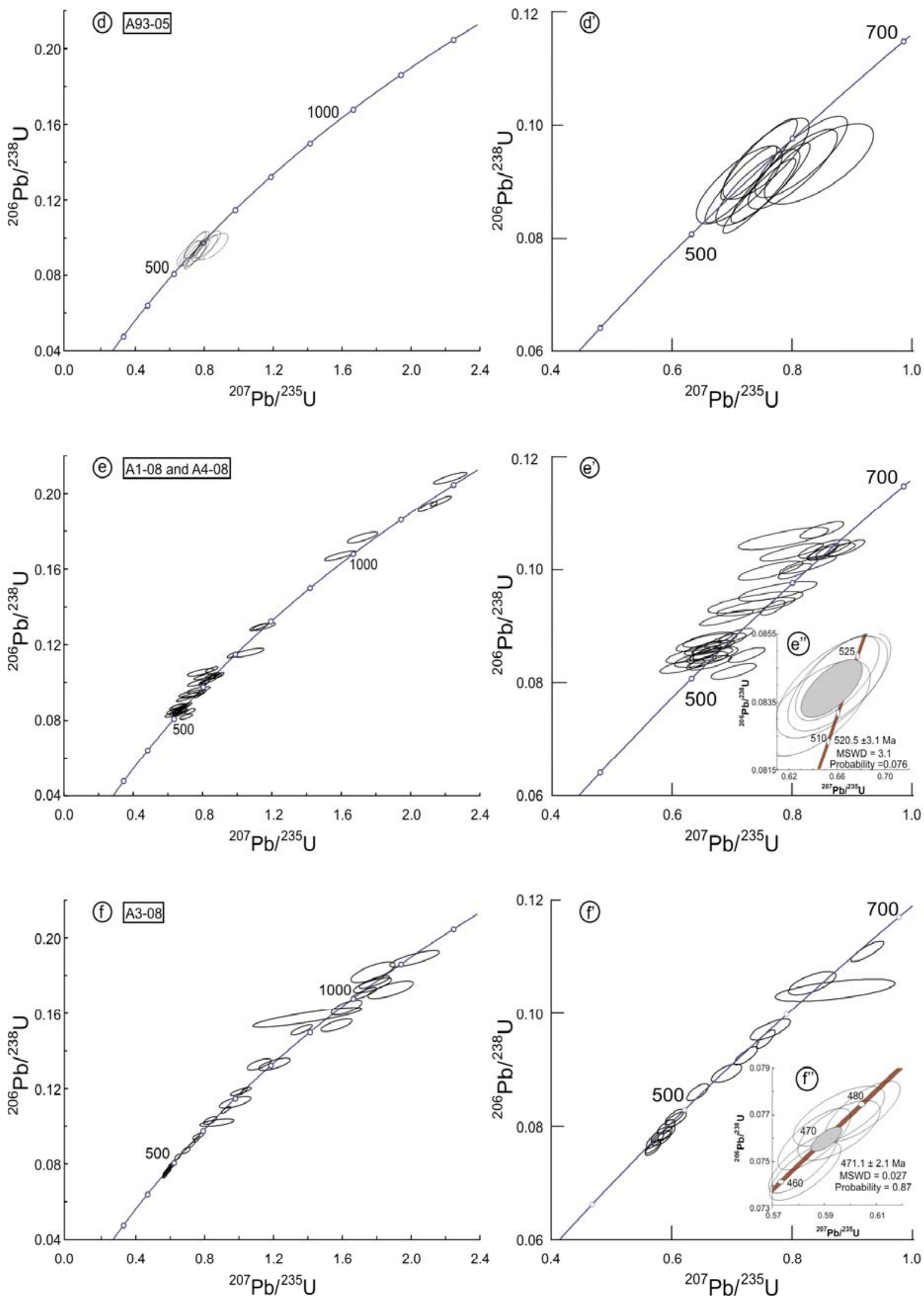

Abbildung 20: Wetherill Concordia Diagramme der Proben (d) A93-05 aus der San Luis Formation, (e) A1-08 und A4-08 aus den Sierras de Córdoba. In Abbildung (e') ist das Maximum um 520 Ma und der weitere sukzessive Verlauf der Alter bis zu ca. $650 \mathrm{Ma}$ sehr gut zu erkennen, bei (e") ist ein konkordantes Alter des Peaks um 520 Ma mit $520.5 \pm 3.1$ Ma dargestellt, (f) A3-08 aus dem südlichsten Bereich der Sierras de Córdoba. Bei ( $\mathrm{f}^{\prime}$ ) ist das famatinische Maximum um 470 Ma besser zu erkennen und in (f') ist das berechnete Alter von 471.1 \pm 2.1 Ma dargestellt. Die Abbildungen d' $\mathrm{f}^{\prime}$ ' beziehen sich auf die gleichen Proben und sind eine Vergrößerung des Altersbereichs bis $700 \mathrm{Ma}$. 
dominiert $(\mathrm{n}=22)$ und ergeben ein Maximum von 530 Ma (Abb. 18 a, 19 a). Die Alter können allerdings auch famatinische Werte von 489 Ma erreichen (1\% diskordant), was mit den Monazitaltern von Steenken et al. (2006) übereinstimmt. Lediglich ein Zirkon deutet auf ein Grenvillian Alter hin, palaeoproterozoische Quellen werden nicht angezeigt. Die nicht zur pampeanen Metamorphose gehörenden Alter über 550 Ma reichen von 730 - $600 \mathrm{Ma}$ und können mit der Brasiliano Orogenese in Verbindung gebracht werden (Leite et al. 2000). Das Fehlen von Altern um 2200 Ma schließt den Río de la Plata Kraton als Liefergebiet aus. Da die Zirkonalter relativ gleichmäßig bis 489 Ma abnehmen, kann keine klare Abgrenzung der pampeanaen und der famatinischen Orogenese getroffen werden. Aufgrund des Fehlens von Altern um 1000 Ma kann von einem unterschiedlichen Liefergebiet im Vergleich zu dem Conlara Metamorphic Complex, der Puncoviscana Formation und der Sierras de Córdoba ausgegangen werden. Die Ursache hiefür könnte an einer Barriere liegen, die sich während der pampeanen Orogenese gebildet hat. Die Heraushebung von Gesteinen mit einer dominant pampeanen Alterssignatur könnte zu einer Verdünnung der Brasiliano und Grenvillian Alter geführt haben, ohne dass diese Liefergebiete komplett abgeschnitten waren.

Die detritischen Alter der San Luis Formation lagen in einem Fall (A93-05) zwischen 590 und $550 \mathrm{Ma}$ (Abb. 19 d). Diese Datenpunkte wiesen Diskordanzen von 8 bis $35 \%$ auf $(\mathrm{n}=10)$. Aus diesen Daten ergibt sich ein Mittelwert von 572 Ma. Die jüngsten Alter liegen bei 550 Ma, was als Maximalalter der Sedimentation angenommen werden kann. Problematisch an diesen Daten sind die teilweise hohe Diskordanz und die großen Fehler um $30 \mathrm{Ma}$. In einer zweiten Probe (A30-01) wurden ebenfalls detritische Zirkone analysiert. In diesem Fall liegen 19 Analysen vor, die alle Diskordanzen unter $10 \%$ aufweisen und einen Fehler um $6 \mathrm{Ma}$ haben. Auch in diesem Fall liegt ein scharfes Maximum vor, allerdings bei einem geringeren Alter von $517 \mathrm{Ma}$ (Abb. 19 c, 20 c, c'). Aus fünf Datenpunkten wurde ein konkordantes Alter von $516.3 \pm 2.7 \mathrm{Ma}$ errechnet (Abb. 20 c'"). Beide Proben weisen ein klares Maximum auf, dessen Altersunterschied möglicherweise in unterschiedlichen Liefergebieten begründet sein könnte, die beide ein geologisches Ereignis anzeigen (z.B. die Intrusion eines plutonischen Körpers), allerdings zeitlich versetzt, eins frühpampean um $570 \mathrm{Ma}$, eins spätpampean um 517 Ma. Da die Zirkone beider Proben detritisch sind und die Proben aus der gleichen Einheit stammen, müssen die jüngsten Datenpunkte als Maximalalter der Sedimentation herangezogen werden. Dieses sollte bei $510 \mathrm{Ma}$ anzusiedeln sein, da drei Datenpunkte ein solches Alter anzeigen $(1.1,3.1,17.1)$ und gezeigt wurde, dass die San Luis Formation nie Temperaturen oberhalb der unteren Grünschieferfazies ausgesetzt war (Wemmer et al. in review). Gebauer und Grünefelder (1976) zeigten zwar, dass Zirkone bereits bei geringen 
Temperaturen von etwas über $300{ }^{\circ} \mathrm{C}$ Metamorphosealter anzeigen können, jedoch sind diese Alter mit Bleiverlust verbunden und deutlich diskordant. Da dies zumindest bei A30-01 nicht der Fall ist, kann ein solcher Einfluss auf die Alter dieser Probe ausgeschlossen werden. Da bereits die SHRIMP Daten des Nogolí Metamorphic Complex und des Pringles Metamorphic Complex (Steenken et al. 2006) deutlich weniger Alter der Brasiliano Orogenese und kaum Alter der Grenvillian Orogenese besaßen und sich dies in den beiden Proben der San Luis Formation fortsetzt, eröffnet dies die Möglichkeit, dass die drei Einheiten ein vergleichbares Liefergebiet besaßen. Llambías et al. (2003) berichteten von früh-pampeanem Vulkanismus um $584 \mathrm{Ma}$ in der Sierra Norte. Diese Alter stimmen mit denen von A93-05 überein und können auch im Nogolí Metamorphic Complex (Anhang 4.1) und dem Pringles Metamorphic Complex (Steenken et al. 2006) nachgewiesen werden. Allerdings lassen die stark negativen ${ }_{\varepsilon} \mathrm{Nd}_{(540 \mathrm{Ma})}$ Werte der San Luis Formation Zweifel an einer solchen Provenienz aufkommen, da eine vulkanische Aktivität mit erhöhten $\varepsilon \mathrm{Nd}_{(540 \text { Ma) }}$ Werten in Zusammenhang stehen sollte. Auch wenn nur juveniles vulkanisches Material die $\varepsilon \mathrm{Nd}_{(540 \text { Ma) }}$ Daten erhöht, bliebe bei einem solchen Liefergebiet unklar, warum die San Luis Formation die geringsten $\varepsilon \operatorname{Nd}_{(540}$ Ma) Verhältnisse der gesamten Sierra de San Luis aufweist. Auch die Sm-Nd $\mathrm{T}_{\mathrm{DM}}$ Modellalter sollten jünger sein als in den übrigen metamorphen Einheiten. Eine weitere mögliche Quelle der magmatisch zonierten Zirkone der San Luis Formation könnten plutonische Körper sein. Diese können eine sehr geringe Altersverteilung ihrer Zirkone besitzen und zusätzlich hohe $\mathrm{T}_{\mathrm{DM}}$ Modellalter und geringe $\varepsilon \mathrm{Nd}_{(540 \mathrm{Ma})}$ Daten aufweisen (Las Verbenas Tonalit, Steenken et al. 2004, 2006). Diese Fakten sprechen für einen plutonischen Komplex als Liefergebiet der San Luis Formation. Aufgrund der bimodalen Verteilung der geochemischen Daten des Pringles Metamorphic Complex müsste das Liefergebiet dieser Einheit aus mindestens einer weiteren Quelle bestehen.

Detritische Alter um 520 Ma können nicht nur in der San Luis Formation (A30-01) sondern auch in den Sierras de Chepes nachgewiesen werden (A1-08). Auch hier liegt das stärkste Maximum bei 520 Ma und auch das Maximalalter der Sedimentation dürfte mit dem der San Luis Formation um 510 Ma übereinstimmen. Ein solches Maximum kann bei der zweiten Probe aus den Sierras de Chepes (A4-08) nicht nachgewiesen werden, die älteren Maxima stimmen jedoch überein. Hauptunterschied der Altersverteilungen der Proben aus den Sierras de Chepes im Gegensatz zu den Proben der San Luis Formation sind zusätzliche Maxima zu früh-pampeanen Zeiten um 570 Ma (wie A93-05), Alter der Brasiliano Orogenese mit einem Maximum um 630 Ma, gefolgt von Grenvillian Altern und zwei Datenpunkten bei 2100 Ma. Trotz der geringen Alter um 520 Ma lagen detritische Zirkone vor, da die 
Metamorphosebedingungen der Sierras de Chepes nicht ausgereicht haben, um ein Neuwachstum von Zirkonrändern zu ermöglichen. Lediglich bei zwei Datenpunkten (11.2, 24.1) mit Altern von 501 Ma und 466 Ma könnte ein metamorpher Einfluss vorgelegen haben. Allerdings könnten diese Werte aufgrund ihres leicht diskordanten Charakters von 3 und $8 \%$ als etwas zu gering angenommen werden. Ihre ${ }^{207} \mathrm{~Pb} /{ }^{206} \mathrm{~Pb}$ Alter liegen etwas höher bei 506 und $518 \mathrm{Ma}$, also im Bereich des stärksten Maximums bzw. des Maximalalters der Sedimentation. Wären die Zirkone in den metasedimentären Enklaven von den granitischen Intrusionen in die Sierras de Chepes beeinflusst worden, so müssten sich Alter von $490 \mathrm{Ma}$ ergeben, da Pankhurst et al. (1998) ein solches Intrusionsalter der Granitoide an Zirkonen bestimmt haben. Demzufolge müssten die konkordanten Zirkone der metasedimentären Gesteine um 520 Ma detritisch sein. Ähnliche Maxima von 522 und 519 Ma wurden auch in der Negro Peinado und der Achavil Formation aus dem Famatina Belt datiert (Collo et al. 2009) und können auch in der Mesón Group, die diskordant auf der Puncoviscana Formation liegt, nachgewiesen werden (persönliche mündliche Mitteilung C. Augustsson). All diese Einheiten liegen im Westen der östlichen Sierras Pampeanas und sind mit Ausnahme der grünschiefer- bis amphibolitfaziellen Metasedimente der Sierras de Chepes gering metamorph. Folglich sind all diese spät-pampeanen Alter detritisch. Der Pringles Metamorphic Complex und die San Luis Formation der Sierra de San Luis wurden nach der Hauptphase der pampeanen Orogenese abgelagert, wie bereits bei Steenken et al. (2004) postuliert. Die neuen Daten, die auf ein Maximalalter der Sedimentation von $510 \mathrm{Ma}$ hindeuten, können das synsedimentäre Zirkonalter von $529 \pm 12$ Ma von Söllner et al. (2000) nicht bestätigen, sondern müssen es nach unten korrigieren. Weiterhin könnte die Ähnlichkeit der Alter weiter nördlich, in den Sierras de Chepes, dem Famatina Belt (Collo et al. 2009) und der Mesón Group (persönliche mündliche Mitteilung C. Augustsson) auf ein zusammenhängendes Ereignis für all diese Einheiten im Westen der östlichen Sierras Pampeanas hindeuten. Zusätzlich zu den Maximalaltern datierten Steenken et al. (2006) Ränder von Zirkonen im Pringles Metamorphic Complex auf 498 Ma, was das Minimalalter der Sedimentation angibt. Diese muss also zwischen 510 und 500 Ma stattgefunden haben. Da abgesehen vom Pringles Metamorphic Complex und der San Luis Formation in allen anderen Einheiten auch ein größerer Anteil an Brasiliano und/oder Grenvillian Altern nachgewiesen werden konnte, muss das Liefergebiet dieser Einheiten stärker begrenzt gewesen sein. Die Altersverteilung der übrigen Einheiten ist, abgesehen von dem 520 Ma Altern, vergleichbar mit den Sierras de Córdoba, dem Conlara Metamorphic Complex und der Puncoviscana Formation, die während der pampeanen Orogenese deformiert und intrudiert wurden. Die 
älteren Körner zeigen ererbte Grenvillian Alter um 1000 Ma und Alter der Brasiliano Orogenese um 800 - 600 Ma. Anhand dieser Alter bestehen mögliche Liefergebiete im Süden Brasiliens und am Kalahari Kraton, inklusive der früheren Suturzone zum Río de la Plata Kraton im Osten von Uruguay und Brasilien (Abb. 21 b). Basierend auf Zirkon Altersdatierungen und Sm-Nd Daten haben Schwartz und Gromet (2004), Steenken et al. (2004, 2006), Adams et al. (2006, 2008) und Rapela et al. (2007) bereits Ähnlichkeiten zwischen den Sierras de Córdoba und dem Conlara Metamorphic Complex im Süden und der Puncoviscana Formation im Norden herausgestellt. Die Herkunft der Metasedimente der Sierras de Chepes und des Orthogneises im südlichsten Bereich der Sierras de Córdoba (A308) wurden noch nicht detailliert mit den hier angewendeten Methoden analysiert. Die Unterschiede liegen in den U-Pb Zirkonaltern um 520 Ma in den Sierras de Chepes und dem famatinischen Maximum der Sierras de Córdoba zwischen 475 und 470 Ma, mit einem konkordanten Alter von 471.1 Ma $\pm 2.1 \mathrm{Ma}(\mathrm{n}=5,2 \sigma$, Abb. 20 f' $)$. Dieses junge Alter spiegelt keine detritischen Zirkone oder das Maximalalter der Sedimentation wider, da die metasedimentären Gesteine dort bereits deutlich vor dieser Zeit deformiert wurden. Zusätzlich sind die Th/U Verhältnisse der Alter unter 500 Ma extrem niedrig $(<0.015)$. Solch junge Alter kommen auch in der Sierra Norte vor und wurden dort als hydrothermales Ereignis diskutiert (Leal et al. 2003; Siegesmund et al. 2009), können aber auch im Nogolí Metamorphic Complex (Monazite) und im Pringles Metamorphic Complex an Zirkonen nachgewiesen werden (Steenken et al. 2006). Außerdem publizierten Pankhurst et al. (1998) Rb-Sr Isochronen um $470 \mathrm{Ma}$ aus den Sierras de Chepes. Im Conlara Metamorphic Complex konnten solche famatinischen Alter nicht nachgewiesen werden. Dieses junge, famatinische Ereignis könnte ebenso mit magmatischer Aktivität in den Sierras de Córdoba in Zusammenhang stehen. Siegesmund et al. (2009) publizierten Zirkonalter aus dem San Carlos Migmatit, die ein konkordantes Alter von $496 \pm 9$ Ma ergaben und ein vergleichbares Alter von $492 \pm$ Ma aus der Sierra Norte. Die Hauptphase der magmatischen Aktivität in den Sierras de Córdoba und der Sierra Norte fand zwischen 555 - 525 Ma statt (Rapela et al. 1998; Stuart-Smith et al. 1999; Söllner et al. 2000; Leal et al. 2003; Llambias et al. 2003; Schwartz et al. 2008). Siegesmund et al. (2009) fanden zusätzlich ältere Körper, wie den Cañada del Sauces Diatexit, der ein konkordantes Alter bei $577 \pm 11$ Ma ergab. Wenn sich dieses Alter auf eine Intrusion bezieht, so steht dies im Widerspruch zu vorherigen Modellen. Alter um 570 Ma kommen auch in den Sierras de Chepes $(n=3$; A1-08 und A4-08) und im Süden der Sierras de Córdoba vor $(n=3$; A3-08), scheinen jedoch zumindest im Fall der Sierras de Chepes detritisch zu sein, weil dort keine frühere Hochtemperaturmetamorphose 
nachgewiesen wurde und die Intrusion zu einem späten Zeitpunkt stattfand (Dahlquist et al. 2005).

Die Altersverteilung der Zirkone im nördlichen Bereich der Sierras de Córdoba (Schwartz und Gromet 2004) und des Conlara Metamorphic Complex (Steenken et al. 2006; diese Arbeit) und des südlichen Bereichs der Sierras de Córdoba ist sehr ähnlich, auch wenn hier ein Orthogneis vorliegt. Alle Proben haben Maxima bei $580 \mathrm{Ma}, 630 \mathrm{Ma}$, um $700 \mathrm{Ma}$, bei 800 Ma, um 1000 Ma und einige Alter zwischen 2100 und 1800 Ma. Adams et al. (2006, 2008) publizierten Zirkondaten der Puncoviscana Formation mit ähnlichen Maxima. Auf der Basis dieser Daten wäre es möglich die Sierras de Córdoba und den Conlara Metamorphic Complex als höhergradig metamorphes Äquivalent der Puncoviscana Formation anzusehen. Allerdings zeigten Adams et al. (2008) ebenso zwei Proben mit einem unterschiedlichen Maximum bei 530 - $520 \mathrm{Ma}$, ähnlich zu den detritischen Altern der Mesón Group und in der südlichen Verlängerung liegenden Bereichen, wie dem Famatina Belt, den Sierras de Chepes und der San Luis Formation. Solche jungen Alter stehen im Widerspruch $\mathrm{zu}$ U-Pb Zirkon Intrusionsaltern von $536 \pm 7 \mathrm{Ma}$ und $534 \pm 9 \mathrm{Ma}$, die an syn- bis postorogenen Kalkalkaligraniten beschrieben wurde (Bachmann et al. 1987). Ein weiteres Problem stellt der Santa Rosa de Tastil Batholith westlich der Stadt Jujuy dar, der bei Hongn et al. (2001) beschrieben ist. Diese Autoren gaben ein Intrusionsalter zwischen 525 - $520 \mathrm{Ma}$ an und diskutierten den Kontakt des Granits mit der Mesón Group und der Puncoviscana Formation als intrusiv. Dies würde bedeuten, dass die Mesón Group älter als 525 Ma ist. Da die Mesón Group aufgrund eines diskordanten Kontakts zur Puncoviscana Formation definitiv jünger als diese ist, steht das Alter des Granits im Widerspruch zu dem Maximum der detritischen Zirkone bei Adams et al. (2008) von 520 Ma. Das gut ausgeprägte Maximum bei 520 Ma ist ebenso ein starkes Argument, dass die Sierras de Córdoba ungefähr 50 Ma älter sind (Cañada del Sauces wurde auf 577 Ma datiert) als die Puncoviscana Formation. Trotzdem besteht bei einem Blick auf die geodynamische Situation die Möglichkeit, diese beiden Einheiten miteinander zu verknüpfen. 


\section{Zusammenfassung und Schlussfolgerungen}

\subsection{Geodynamische Modelle}

\subsubsection{Mögliche Liefergebiete der (Meta)Sedimente der östlichen Sierras Pampeanas}

Die vorliegenden neuen Informationen aus Isotopensystemen wie Sm-Nd, der Pb-Isotopie und U-Pb SHRIMP Daten von Zirkonen, zusammen mit Literaturdaten (z.B. Sims et al. 1998; Rapela et al. 1998, 2007; Schwartz und Gromet 2004; Steenken et al. 2004, 2006; Adams et al. 2008; Collo et al. 2009) sprechen klar gegen den Río de la Plata Kraton als Hauptliefergebiet der metasedimentären Einheiten der östlichen Sierras Pampeanas, obwohl sie heute in direktem Kontakt zueinander stehen.

Detritische Zirkone der Puncoviscana Formation haben ihre stärksten Maxima bei Altern von 680 - 570 Ma und um 1000 Ma. Zusätzliche kleinere Populationen zwischen den vorher genannten Gruppen decken die komplette Breite der Panafrikanisch - Brasiliano Orogenese ab. Ältere Datenpunkte kommen lediglich untergeordnet vor (Adams et al. 2006, 2008). Diese Altersverteilung ist der in den Sierras de Córdoba (Schwartz und Gromet 2004) und dem Conlara Metamorphic Complex ähnlich (Steenken et al. 2006). Die Brasiliano und Grenvillian Alter kommen in Südbrasilien, in Orogenen östlich des Río de la Plata Kratons und um den Kalahari Kraton wie dem Damara Orogen, dem Ostantarktis Orogen, dem Namaqua-Natal Belt und dem Dom Felician-Gariep Belt vor (Scheepers und Armstrong 2002; Basei et al. 2005; Eglington 2006). Diese Orogene wurden nach der Schließung des Adamaster Ozeans in Kontakt zueinander gebracht (Abb. 24 a). Dabei kollidierten der Río de la Plata Kraton, der Kongo Kraton und der Kalahari Kraton (z.B. Chemale et al. 1995; Frimmel et al. 1996; Rozendaal et al. 1999; Basei et al. 2005). Die Kollision und die darauf folgende Hebung setzte die Gesteine der Erosion aus und die daraus entstehenden Sedimente wurden in das Puncoviscana Becken transportiert (Abb. 24 b). Anschließend wurden die in dem Becken abgelagerten Sedimente anhand dextraler Scherzonen neben den Río de la Plata Kraton verschoben (Rapela et al. 2007; Casquet et al. 2008). Dieses Modell beinhaltet ebenso die Exhumation von Grenvillian geprägten Gesteinen entlang des „Westrandes“ des Amazonia Kratons wie z.B. den Sunsas Belt (Cordani et al. 2000), ausgelöst durch die Kollision von Amazonia mit Laurentia (Abb. 24 c, d). Das Basement der Östlichen Sierras Pampeanas, auf welches der Detritus mit Grenvillan und Brasiliano Zirkonaltern abgelagert wurde, müsste nach diesem Modell vom Kalahari Kraton stammen (Rapela et al. 2007). Dies 

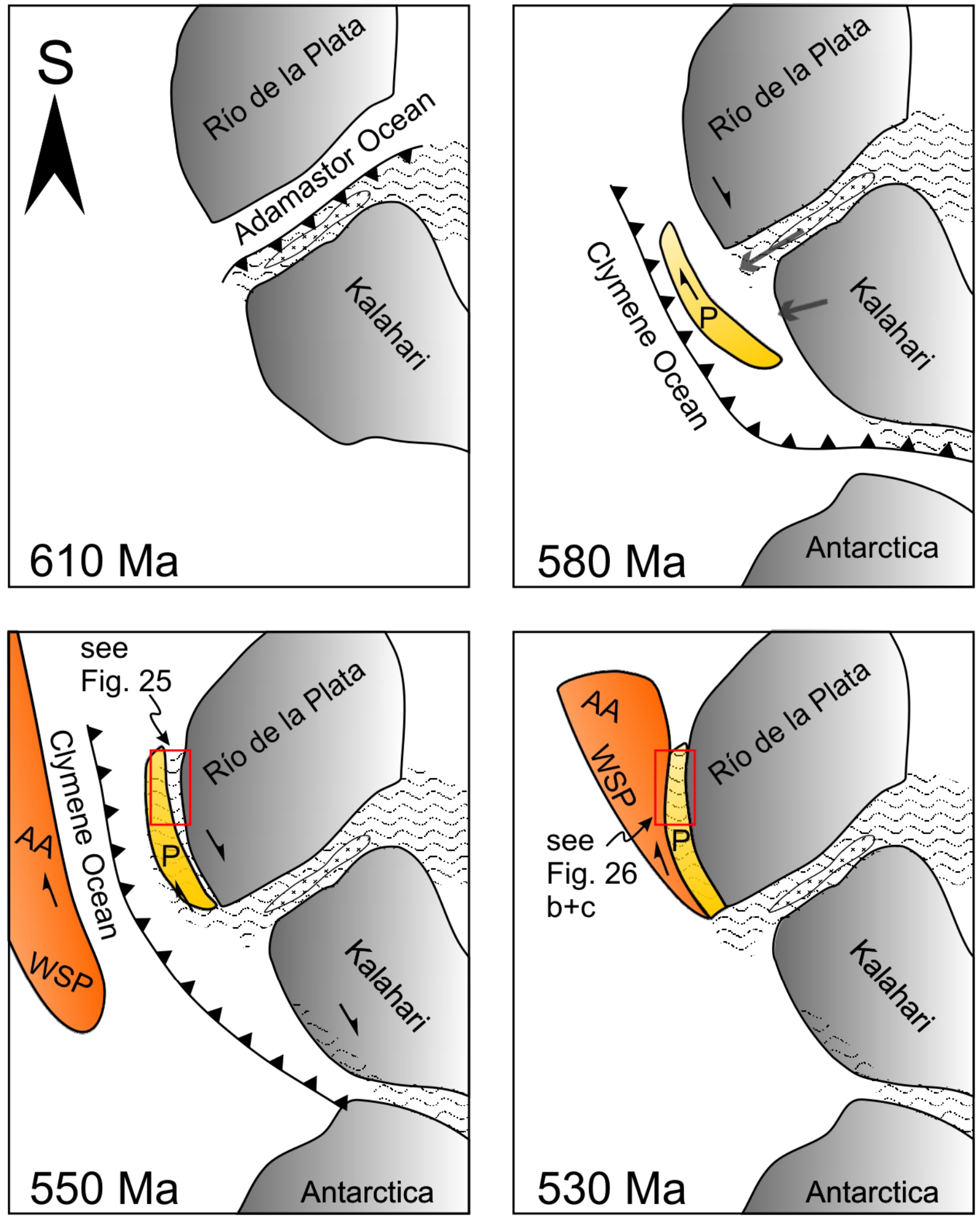

Abbildung 24: Zeichnung der Bildung Westgondwanas zwischen 610 und $530 \mathrm{Ma}$ (verändert nach Rapela et al. 2007). (a) Der Río de la Plata Kraton nähert sich dem Kalahari Kraton. Dies ist mit einer Subduktionszone unter dem Kalahari Kraton und der Bildung eines magmatischen Bogens verbunden. (b) Nach der Kollision der Kratone und der Heraushebung des magmatischen Bogens wurde das erodierte Material (graue Pfeile) auf das pampeane Terrane (P, gelb) geschüttet. (c) Dieses Terrane wurde entlang dextraler Scherzonen neben den Río de la Plata Kraton transportiert. Um 550 Ma näherte sich eine große Landmasse, die u. a. aus dem ArequipaAntofalla Kraton (AA) und den westlichen Sierras Pampeanas (WSP) bestand (orangefarbener Bereich) und die Subduktion unter den Río de la Plata Kraton begann, bis die Landmasse aus Arequipa-Antofalla Kraton und westlichen Sierras Pampeanas schließlich (d) um 530 Ma mit dem Río de la Plata Kraton kollidierte. 
ist zumindest fraglich, da das Basement unterhalb der östlichen Sierras Pampeanas nicht aufgeschlossen ist. Der Clymene Ozean begann sich um ca. $770 \mathrm{Ma}$ zu öffnen und separierte Proto-Westgondwana vom Amazonia Kraton (Trindade et al. 2006). Diese Bewegung wurde vor ca. $550 \mathrm{Ma}$ umgekehrt. Ursache hierfür war die Annäherung einer kontinentalen Landmasse, die den Amazonia Kraton, den Arequipa-Antofalla Kraton und die westlichen Sierras Pampeanas umfasste, dextral mit dem Río de la Plata Kraton und dem Kalahari Kraton kollidierte und die pampeane Orogenese auslöste (Abb. 24 d). Mögliche Liefergebiete für die Sierras de Córdoba, den Conlara Metamorphic Complex und die Puncoviscana Formation mit ihrer bimodalen Altersverteilung könnten der Dom Feliciano Belt, z.B. der granitische Floriaópolis-Pelotas-Aiguá Batholith sein. Obwohl die Deformation, die Metamorphose und die Intrusion magmatischer Körper der genannten Liefergebiete vor $600 \mathrm{Ma}$ stattfand, kommen die detritischen Zirkone immer zusammen mit Grenvillian Altern vor (Basei et al. 2000; Preciozzi et al. 2001; Adams et al. 2008).

\subsubsection{Geodynamische Entwicklung der Sierras de Córdoba und der Puncoviscana Formation}

Auf Abbildung 25 a ist ein Gesamtüberblick über das Untersuchungsgebiet um $580 \mathrm{Ma}$ gegeben. Der scheinbare Widerspruch zwischen den Intrusionsaltern (Bechmann et al. 1987; Hongn et al. 2001) und den detritischen Zirkonaltern der Puncoviscana Formation könnte durch eine bereits von Zimmermann (2005) aufgestellte Vermutung erklärt werden. Dieser brachte die Idee eines kannibalistischen Recyclings der Puncoviscana Formation auf. Dabei werden die Sedimente während der Subduktion am aktiven Kontinentalrand Gondwanas in ein Vorland-Becken geschüttet. Die Sedimente können während dieses Prozesses aufgrund der anhaltenden Subduktion bereits gefaltet, gehoben und möglicherweise bereits von magmatischen Prozessen beeinflusst werden (Abb. 25 b, b'). Die gefalteten und teilweise exhumierten Sedimente könnten zusammen mit lokalen Intrusionen erneut in das Vorland sedimentiert werden, in welches sie bereits zuvor abgelagert wurden (Abb. 25 c, c'). Aufgrund der Subduktion eines ozeanischen Rückens (Schwartz et al. 2008; Siegesmund et al. 2009) im Bereich der Sierras de Córdoba und der Sierra Norte wurde die Temperatur erhöht. Dies könnte den hochgradigen Metamorphismus in den Sierras de Córdoba und den kambrischen Plutonismus in der Sierra Norte ausgelöst haben (Abb. 25 c, d). Gleichzeitig wurde der Bereich der Puncoviscana Formation lediglich niedriggradig metamorph überprägt (Abb. 25 c'). Aufgrund der anhaltenden Subduktion kam es zu lokalen Granitintrusionen 
580-550 Ma

(a) Sedimentation in arc related basins and magmatism

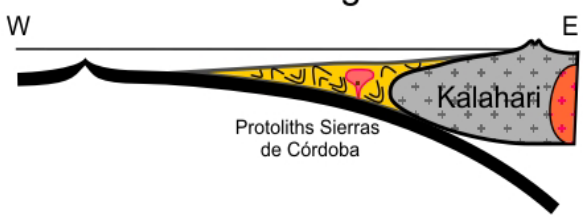

550 - $540 \mathrm{Ma}$ (b) Oblique subduction of the trench

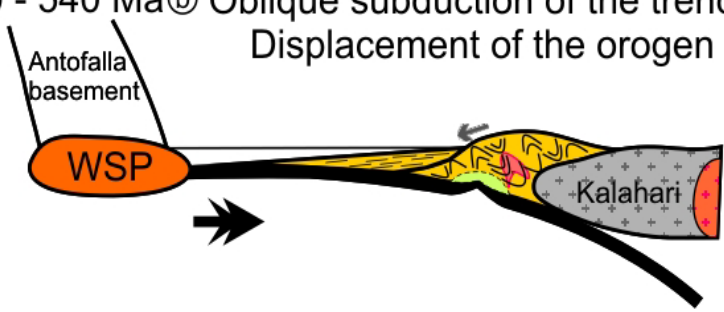

$530 \mathrm{Ma}$

(C) Accretion/collision and final closure of the orogen

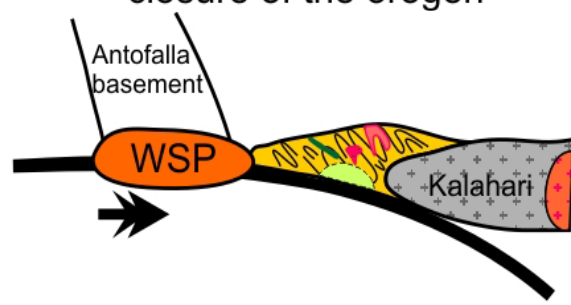

$520 \mathrm{Ma}$

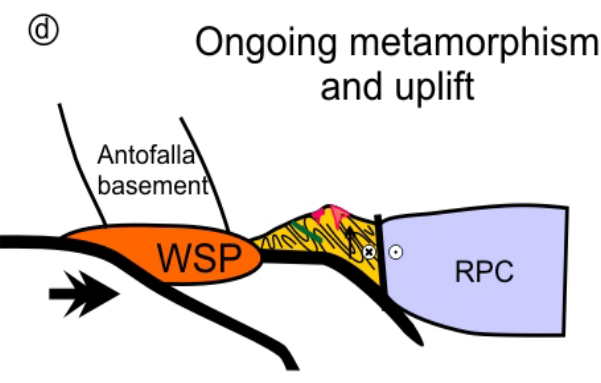

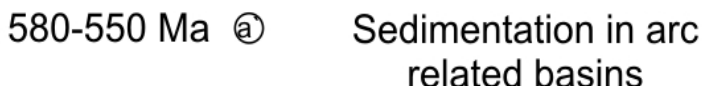

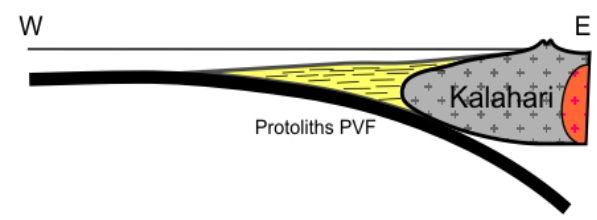

550 - $540 \mathrm{Ma}(b)$ Ongoing folding and coeval

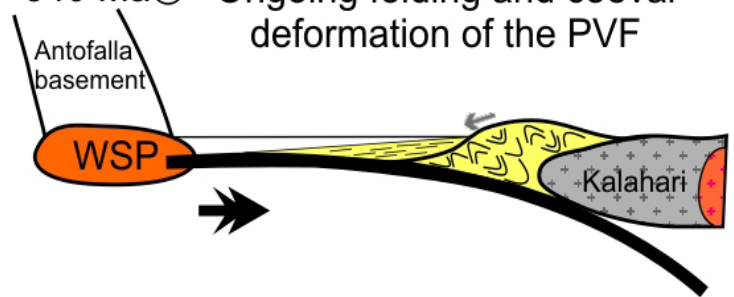

$530 \mathrm{Ma}$ C) Closing of the basin and first magmatism

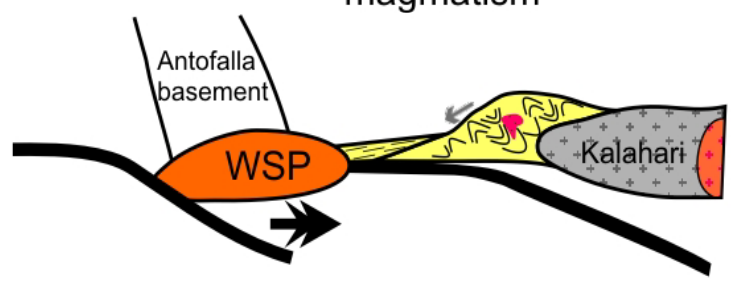

$520 \mathrm{Ma}$

(d) Final deposition into the basin. Youngest ages by volcanites

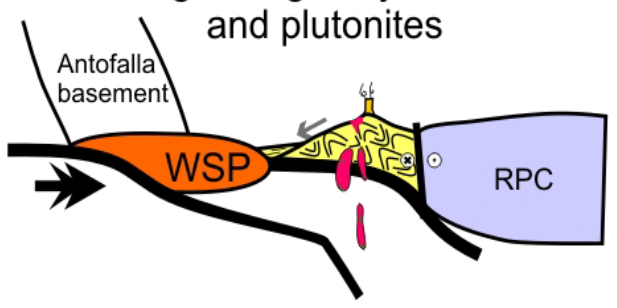

Abbildung 25: Modell zur Bildung Westgondwanas (verändert nach Rapela et al. (2007) und Siegesmund et al. (2009) zwischen 580 und 520 Ma im Bereich der Sierras de Córdoba (b - b') und der Puncoviscana Formation (b' - e'). (a) Überblick über die Paläogeographischie am Westrand Gondwanas um $580 \mathrm{Ma}$. Die Abbildung ist in einen rechten und einen linken Abschnitt unterteilt, um die unterschiedliche Entwicklung der Sierras de Córdoba im Süden und der Puncoviscana Formation im Norden $\mathrm{zu}$ dokumentieren. (b und b') Während die Ausgangsgesteine der Sierras de Córdoba bereits gefaltet und von granitoiden Körpern intrudiert wurden, sind noch keine Anzeichen einer Faltung oder Metamorphose in den Ausgangsgesteinen der Puncoviscana Formation zu erkennen. (c und c') Die sich fortsetzende Subduktion und Sedimentation führte zur Deformation und Hebung der Sedimente im fore-arc und einer erneuten Sedimentation (Resedimetation) der bereits gehobenen Bereiche in das fore-arc Becken. (d und d') Die Subduktion eines mittelozeanischen Rückens (Schwartz et al. 2008) verursachte einen großen Wärmeeintrag (hellgrün) in den Sierras de Córdoba. Während die Subduktion des Rückens eine hochgradige Metamorphose, Migmatisierung und die Ausbildung (ultra)mafischer Linsen in den Sierras de Córdoba zur Folge hatte wurde die Puncoviscana Formation vereinzelt von granitoiden Körpern intrudiert. Die Resedimentation der for-arc (Meta)Sedimente findet weiterhin statt. (e und e") Um 520 Ma ist das Becken im Süden geschlossen. Die metamorphe Sierras de Córdoba wird nun gehoben (e) und dient als Liefergebiet z.B. für die San Luis Formation und den Pringles Metamorphic Complex (Abb. 26). Im Norden ist das Becken noch nicht ganz geschlossen. Vulkanisches Material und/oder Granitoide, die bereits der Erosion ausgesetzt sind könnten die Quellen der jüngsten detritischen Zirkone von $520 \mathrm{Ma}$ (Adams et al. 2008) in der Puncoviscana Formation sein. 
(Abb. 25 d'). Eine solche geodynamische Begebenheit wäre mit der heutigen indonesischen Westküste $\mathrm{zu}$ vergleichen (Sumatra). In dem dortigen Gebiet ist keine Tiefseerinne zu erkennen (diese beginnt erst weiter südöstlich), da die Sedimentationsraten zu hoch sind. Dies führt zu einer Hebung des Akkretionskeils über den Meeresspiegel und dessen Erosion vor dem vulkanischen Bogen. Die Sedimente der Puncoviscana Formation könnten in ähnlicher Weise gehoben und dabei zusätzlich von vulkanischer Tätigkeit beeinflusst worden sein. Diese vulkanische Tätigkeit wäre dann die Erklärung für die von Adams et al. (2008) beschriebenen Alter von 520 Ma. Eine weitere mögliche Quelle für diese Zirkone könnten spätpampeane Intrusionen wie der Santa Rosa de Tastil Batholith (westlich der Stadt Jujuy) mit einem Intrusionsalter um 525 Ma (Hongn et al. 2001) sein. Solche Intrusionen könnten in einem solchen geologischen Regime exhumiert worden sein und anschließend als Quelle für die jungen Zirkone gedient haben (Abb. 25 e, e'). Während die Aufschlüsse der Puncoviscana Formation, wie wir sie heute sehen, lediglich schwach metamorph beeinflusst wurden, haben die Aufschlüsse der Sierras de Córdoba und des Conlara Metamorphic Complex ihren Ursprung in wesentlich tieferen Krustenstockwerken. Möglicherweise lagen Gesteine ähnlicher Metamorphosegrade wie die der Puncoviscana Formation über den heutigen Aufschlüssen der Sierras de Córdoba und dem Conlara Metamorphic Complex, wurden aber bereits erodiert. Die tieferkrustalen Bereiche, die wir heute sehen, konnten nicht mehr von spätpampeanen detritischen Zirkonen um 520 Ma beeinflusst werden, weil sie bereits zu tief in der Kruste versenkt worden waren, als die Zirkone in der Puncoviscana Formation abgelagert wurden. Aufgrund einer stärkeren Heraushebung und Erosion der südlichen Bereiche der östlichen Sierras Pampeanas liegen in den Sierras de Córdoba und dem Conlara Metamorphic Complex höhere Metamorphosealter und ältere Intrusionen vor als in der Puncoviscana Formation.

\subsubsection{Spät-pampeane Entwicklung der östlichen Sierras Pampeanas}

Nach der Ablagerung und Deformation der Sierras de Córdoba, des Conlara Metamorphic Complex und der Puncoviscana Formation kam es zu einer Dekompression im pampeanen Orogen (Baldo et al. 1996; Rapela et al. 1998; Sims et al. 1998), die zu einer Extension führte und welche die mittel- bis spätkambrischen Becken der östlichen Sierras Pampeanas bildete, in welche der Pringles Metamorphic Complex und die San Luis Formation sedimentiert wurden. Anschließend wurden diese Becken durch die beginnende famatinische Subduktion, die entlang des Westrandes des pampeanen Orogens begann, zumindest teilweise in Back-Arc 


\section{$515 \mathrm{Ma}$}

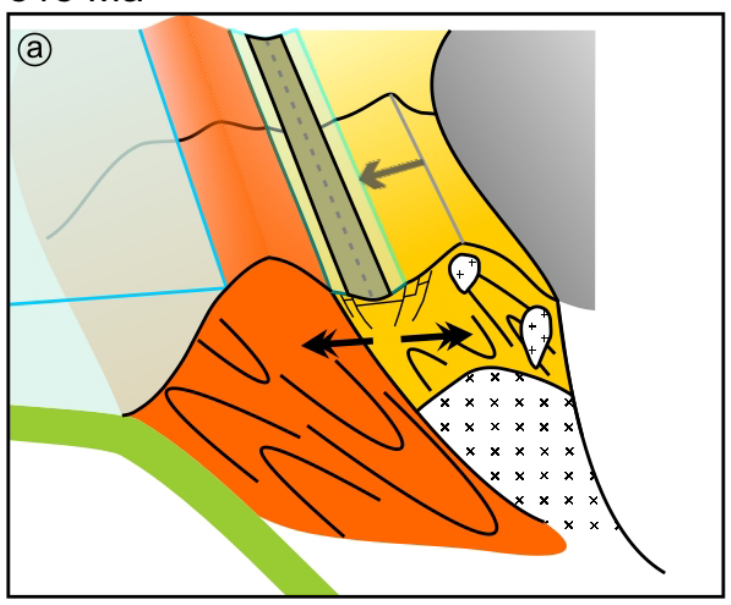

$500 \mathrm{Ma}$

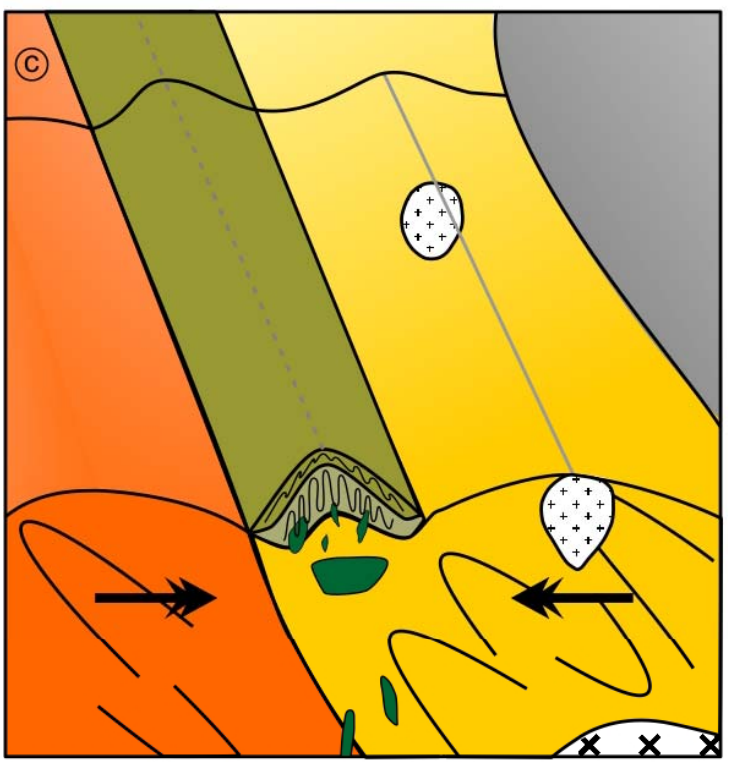

\section{$510 \mathrm{Ma}$}

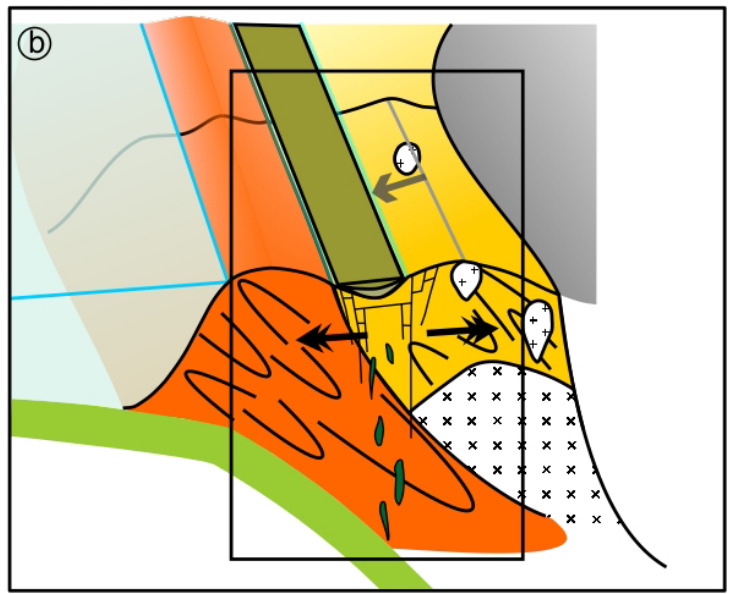

After uplift and erosion

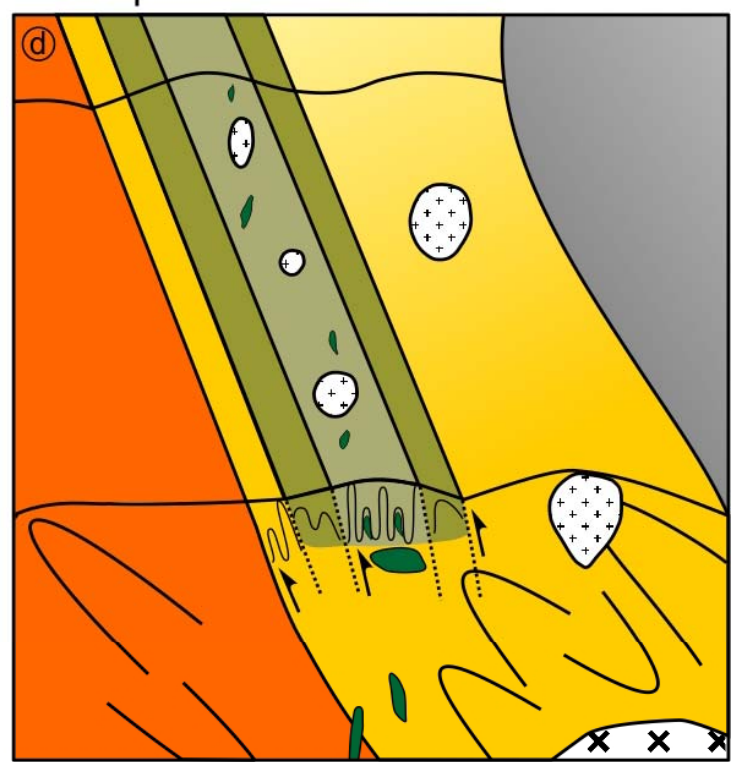

Abbildung 26: (a) Aufgrund von Subduktionsprozessen unter die westlichen Sierras Pampeanas (orange) nach der Kollision mit den östlichen Sierras Pampeanas (gelb) wird eine back-arc Becken zwischen westlichen und östlichen Sierras Pampeanas gebildet. Dieses Becken wird von Sedimenten des neuen pampeanen Orogens gefüllt, aus denen später der Pringles Metamorphic Complex (dunkles Olivgrün) entsteht. (b) Die sich fortsetzende Subduktion und die weitere Öffnung des back-arc Beckens führt zum Aufstieg (ultra)mafischer Schmelze (dunkelgrün; Hauzenberger et al. 2001), während gleichzeitig die San Luis Formation (dunkles Olivgrün) abgelagert wird. (c) Nach dem Ende bzw. der Rückverlagerung der Subduktion wird das Becken geschlossen und die abgelagerten Sedimente werden verfaltet. Aufgrund der Temperatur der (ultra)mafischen Intrusiva wurde im Bereich des Pringles Metamorphic Complex eine amphibolit- bis granulitfazielle Metamorphose ausgelöst. Die San Luis Formation wurde davon geringer beeinflusst, da sie sich weiter höher krustalen Bereichen befand. (d) Nach Hebung und Erosion dieses Gebiets liegen zwei niedrigmetamorphe Abschnitte der San Luis Formation direkt östlich und westlich des hochgradig metamorphen Pringles Metamorphic Complex.

Becken umgewandelt. In einem solchen Becken befinden sich die Mesón Group, der Famatina Belt (Collo et al. 2009), die Sierras de Chepes, der Pringles Metamorphic Complex und die San Luis Formation, die alle Sedimentationsalter unter 520 Ma anzeigen. Collo et al. (2009) gehen davon aus, dass die Protolithe der San Luis Formation und des Conlara Metamorphic Complex vor der Negro Peinado und der Achavil Formation abgelagert wurden. Aufgrund der hier vorgelegten identischen Alter ist davon auszugehen, dass dies gleichzeitig 
geschah. Aufgrund der LP/HT Metamorphose und der (ultra)mafischen Körper im Pringles Metamorphic Complex (Hauzenberger et al. 2001) wird von einer dünnen Kruste ausgegangen, was für ein Back-Arc Becken als Sedimentationsraum des Pringles Metamorphic Complex spricht. Für die (ultra)mafischen Gesteine wurden Alter zwischen 500 und $480 \mathrm{Ma}$ berechnet (Sims et al. 1998; Steenken et al. 2008), was zeigt, dass die Extensionsprozesse und die Sedimentation, zumindest im Bereich der Sierra de San Luis, nach der pampeanen Orogenese stattgefunden haben. Der Einfluss von Altern um $1000 \mathrm{Ma}$ im Nogolí Metamorphic Complex, dem Pringles Metamorphic Complex und der San Luis Formation ist zu vernachlässigen. Dies deutet auf ein verändertes Liefergebiet hin, welches allerdings ähnliche Sm-Nd Werte liefert. Eine mögliche Erklärung könnte eine aufgrund der pampeanen Orogenese entstandene Barriere sein, die den Eintrag von Altern der Grenvillian Orogenese behinderte und selber Alter zwischen 730 - 510 Ma lieferte, die im Nogolí Metamorphic Complex, dem Pringles Metamorphic Complex und der San Luis Formation vorherrschen (Sims et al. 1998; Steenken et al. 2006). Weiterhin besteht die Möglichkeit, dass die Grenvillian Alter durch die frisch exhumierten Gesteine der pampeanen Orogenese so stark verdünnt wurden, dass sie in den Hintergrund treten oder ganz fehlen. Das Altersspektrum der detritischen Zirkone nimmt in diesen Einheiten ab und ist in der San Luis Formation mit einem sehr deutlichen Maximum um 570 Ma (A93-05) und einem bei 517 Ma (A30-01) am geringsten.

Die unterschiedlichen Metamorphosebedingungen der schwach metamorphen San Luis Formation, die sowohl östlich, als auch westlich des amphibolit- bis granulitfaziellen Pringles Metamorphic Complex aufgeschlossen ist, blieb bislang ein ungelöstes Problem. Durch die Annahme einer Sedimentation in ein Back-Arc Becken (Abb. 26 a) könnte dieses Problem gelöst werden, wobei der Pringles Metamorphic Complex als erster abgelagert würde und anschließend die San Luis Formation. Das Back-Arc Becken führte zu den bei Hauzenberger et al. (2001) beschriebenen (ultra)mafischen Intrusionen (Abb. 26 b). Diese Intrusionen fungierten anschließend als Wärmequellen für die amphibolit- bis granulitfazielle Metamorphose im Pringles Metamorphic Complex. Da die San Luis Formation darüber abgelagert wurde, war der Wärmeeinfluss auf sie geringer, als im Pringles Metamorphic Complex. Nach der extensionellen Phase wurde das Becken wieder geschlossen und die (Meta)Sedimente wurden verfaltet und gehoben (Abb. 26 c). Die nachfolgenden Erosionsprozesse legen abschließend hochgradig metamorphe Gesteine im Zentrum frei, den Pringles Metamorphic Complex, östlich und westlich eingerahmt durch niedriggradig metamorphe Gesteine, die San Luis Formation (Abb. 26 d). Die Sedimentation muss 
zwischen 510 und 498 Ma stattgefunden haben, da detritische Zirkone der San Luis Formation dies als Maximalalter angeben und metamorph gewachsene Zirkonränder aus dem Pringles Metamorphic Complex auf $498 \pm 10$ Ma datiert wurden (Steenken et al. 2006).

\subsection{Schlussfolgerungen}

- Geochemische Untersuchungen der unterschiedlichen Einheiten der östlichen Sierras Pampeanas lassen auf einen aktiven Kontinentalrand oder einen kontinentalen Inselbogen als geodynamische Position schließen, ohne eine Aussage treffen zu können, ob es sich um ein Fore-Arc oder ein Back-Arc handelt. Der Pringles Metamorphic Complex ist gegenüber den übrigen Einheiten an Th angereichert, was für ein stärker felsisches Liefergebiet spricht. Dieses Gebiet zeigt außerdem die größte Streuung der geochemischen Daten und lässt auf ein gemischtes Liefergebiet zum einen aus einer stärker aufgearbeiteten Quelle, möglicherweise einem passiven Kontinentalrand und zum anderen aus einer relativ frischen, felsischen Quelle schließen. Dies kann ebenso in den verschiedenen Spurenelementdiagrammen zur Ermittlung des geotektonischen Milieus beobachtet werden. Der Großteil der Proben liegt in den Feldern, die auf einen aktiven Kontinentalrand oder einen kontinentalen Inselbogen hinweisen, außer bei den Proben des Pringles Metamorphic Complex, die ebenso im Feld für einen passiven Kontinentalrand liegen. Eine solche bimodale Verteilung kann auch bei den Hauptelementen beobachtet werden. Dies führt zur Schlussfolgerung, dass der Pringles Metamorphic Complex ein von den anderen Einheiten abweichendes, bimodales Liefergebiet bzw. zwei unterschiedliche Liefergebiete aufweist. Bei den Proben der Sierras de Córdoba fällt auf, dass diese einen stärkeren vulkanischen Einfluss zu haben scheinen, da fast $50 \%$ der Analysen ein $\mathrm{Th} / \mathrm{Sc}$ Verhältnis unter 0.8 aufweisen. In den Diagrammen zur Ermittlung der geotektonischen Position sind die Datenpunkte dieser Einheit tendenziell stärker zum Feld für einen ozeanischen Inselbogen verschoben. Dies gilt auch für die Hauptelemente. Die Daten der Sierras de Córdoba sind mit Literaturwerten des Conlara Metamorphic Complex vergleichbar, die ebenfalls auf einen stärkeren vulkanischen Einfluss in diesem Gebiet hindeuten.

- Ähnlich den vulkanisch/mafischen Tendenzen der Geochemie zeigen die Sierras de Córdoba die geringsten $\mathrm{T}_{\mathrm{DM}}$ Modellalter und die höchsten $\varepsilon_{\mathrm{Nd}}$ Werte an. Diese sind nur mit einigen Werten aus dem Conlara Metamorphic Complex vergleichbar. Auf der anderen Seite fallen die San Luis Formation, die Sierras de Chepes und die Sierra Norte durch überdurchschnittlich hohe $\mathrm{T}_{\mathrm{DM}}$ Modellalter und geringe $\varepsilon_{\mathrm{Nd}}$ Verhältnisse auf und sind mit 
denen des Famatina Belt vergleichbar. Abgesehen von der Sierra Norte, wo es nicht nachgewiesen ist, wurden alle Einheiten mit geringen $\varepsilon_{\mathrm{Nd}}$ Werten und hohen $\mathrm{T}_{\mathrm{DM}}$ Modellaltern nach den Sierras de Córdoba und dem Conlara Metamorphic Complex abgelagert. Auch wenn die Puncoviscana Formation etwas höhere $\mathrm{T}_{\mathrm{DM}}$ Modellalter und geringere $\varepsilon_{\mathrm{Nd}}$ Verhältnisse als z.B. die Sierras de Córdoba vorweisen kann, so erreichen diese immer noch nicht die der San Luis Formation und der Sierras de Chepes.

Ähnlich zu früheren Untersuchungen liegen die $\mathrm{T}_{\mathrm{DM}}$ Modellalter der Sierras de Córdoba um $1.6 \mathrm{Ga}$ und die $\varepsilon_{\mathrm{Nd}}$ Werte zwischen -5 bis -6 , allerdings kommen auch hier zwei Proben mit deutlich älteren $\mathrm{T}_{\mathrm{DM}}$ Werten von $1.9 \mathrm{Ga}$ und geringeren $\varepsilon_{\mathrm{Nd}}$ Daten von -6 bis -5 vor. Auch dies ist mit dem Conlara Metamorphic Complex vergleichbar, wenn nicht nur die neuen, sondern auch die Literaturwerte hinzugezogen werden. Auch hier scheint sowohl stark entwickeltes als auch relativ primitives Material vorzuliegen. Auch wenn in den Sierras de Córdoba eine große Variation der Daten vorliegt, so kann keine Tendenz entlang eines Profils ausgemacht werden.

- Die ${ }^{207} \mathrm{~Pb} /{ }^{204} \mathrm{~Pb}$ vs. ${ }^{206} \mathrm{~Pb} /{ }^{204} \mathrm{~Pb}$ Isotopenverhältnisse liegen auf einem linearen Trend. Vergleichbare Daten des nördlichen Bereichs des Arequipa-Antofalla Kratons haben einen steileren Trend, als die hier vorgestellten Daten. Dieser kann somit als mögliches Liefergebiet ausgeschlossen werden. Ein Bereich des Zentralen Kratons (Belén) kann ebenfalls als Liefergebiet ausgeschlossen werden, da die hier vorkommenden ${ }^{208} \mathrm{~Pb} /{ }^{204} \mathrm{~Pb}$ vs. ${ }^{206} \mathrm{~Pb} /{ }^{204} \mathrm{~Pb}$ über den neuen Werten der östlichen Sierras Pampeanas liegen. Eine weitere Aussage, die aufgrund der ${ }^{207} \mathrm{~Pb} /{ }^{204} \mathrm{~Pb}$ vs. ${ }^{206} \mathrm{~Pb} /{ }^{204} \mathrm{~Pb}$ Werte gemacht werden kann, ist, dass auf Liefergebiete in Gondwana geschlossen wird, da die Bereiche in Laurentia geringere $\mathrm{Pb} / \mathrm{Pb}$ Verhältnisse besitzen. Die ${ }^{208} \mathrm{~Pb} /{ }^{204} \mathrm{~Pb}$ vs. ${ }^{206} \mathrm{~Pb} /{ }^{204} \mathrm{~Pb}$ Werte der untersuchten Gesteine zeigen eine größere Variabilität der Datenpunkte in den Sierras de Córdoba und tendieren zu höheren ${ }^{208} \mathrm{~Pb} /{ }^{204} \mathrm{~Pb}$ Werten im Pringles Metamorphic Complex, was aufgrund der erhöhten Th Konzentration zu erwarten war. Vier Proben der Sierras de Córdoba und die Metaarkose mit den vulkanischen Klasten aus der Sierra Norte zeigen ebenfalls ein erhöhtes ${ }^{208} \mathrm{~Pb} /{ }^{204} \mathrm{~Pb}$ Verhältnis an und tendieren zum nördlichen Bereich und zum zentralen Bereich (Belén) des Arequipa-Antofalla Kratons und zur Richtersveld Domain. Zwei weitere Proben mit den am stärksten radiogenen ${ }^{206} \mathrm{~Pb} /{ }^{204} \mathrm{~Pb}$ Verhältnissen liegen dagegen in einem anderen Feld des Zentralbereichs (Choja) und im südlichen Bereich des Arequipa-Antofalla Kratons bzw. des Natal Belts. Die erhöhten ${ }^{208} \mathrm{~Pb} /{ }^{204} \mathrm{~Pb}$ Verhältnisse der Sierras de Córdoba könnten an einem stärkeren vulkanischen Einfluss in diesem Gebiet liegen, wofür bereits Hinweise aus der Geochemie und der Nd-Isotopie existieren oder ihre Ursache in generell erhöhten ${ }^{208} \mathrm{~Pb} /{ }^{204} \mathrm{~Pb}$ 
Werten in der mittleren Erdkruste haben, worauf vorherige Arbeiten anhand von Xenolithen bereits hinwiesen. Ob die (meta)sedimentären Gesteine ihren Ursprung im Brasilianischen Schild, dem zentralen oder südlichen Bereich des Arequipa-Antofalla Kratons oder aber im südlichen Afrika haben kann, nicht eindeutig geklärt werden, da sich die Isotopenverhältnisse gleichen.

- Als Maximalalter der Sedimentation der San Luis Formation und der Sierras de Chepes werden ca. $510 \mathrm{Ma}$ angenommen, da die jüngsten detritischen U-Pb SHRIMP Analysen dieser Einheiten in diesem Bereich liegen. Trotzdem müssen diese beiden Gebiete unterschiedliche Liefergebiete gehabt haben, da die San Luis Formation nur drei Datenpunkte aufweist, die nicht in das Maximum um 517 Ma fallen, während die Altersverteilung der Sierras de Chpes ebenso Alter der Brasiliano und Grenvillian Orogenese und auch zwei palaeoproterozoische Alter beinhaltet. Abgesehen von dem Maximum um 520 Ma haben die Proben aus den Sierras de Chepes ähnliche Altersverteilungen wie der Conlara Metamorphic Complex und die Sierras de Córdoba. Die Probe aus dem Süden der Sierras de Córdoba hat eben solche Verteilungen wie die vorher genannten, allerdings zusätzlich ein famatinisches Maximum um $470 \mathrm{Ma}$, das metamorph ist und nichts mit der Provenienz zu tun hat.

Es wird von einem geologischen Modell mit kannibalitischem Recycling ausgegangen, welches eine Korrelation der Puncoviscana Formation mit den Sierras de Córdoba und dem Conlara Metamorphic Complex ermöglicht. Das Fehlen von Altern um 520 Ma kann in den mittelkrustalen Aufschlüssen in den Sierras de Córdoba und dem Conlara Metamorphic Complex erklärt werden. Eine weitere Möglichkeit besteht darin, dass nicht die richtigen Probenahmeorte für solche Alter gewählt wurden, denn auch in der Puncoviscana Formation wurden diese jungen Alter nicht in allen Proben nachgewiesen.

Eine jüngere Sedimentation fand westlich der Sierras de Córdoba, des Conlara Metamorphic Complex und der Puncoviscana Formation statt. Zu diesen Einheiten zählen die San Luis Formation, der Pringles Metamorphic Complex, der Famatina Belt und die Mesón Group. Diese Einheiten haben nicht nur detritische Zirkone zwischen 520 und 510 Ma, sondern zusätzlich höhere $\mathrm{T}_{\mathrm{DM}}$ Modellalter und geringere $\varepsilon_{\mathrm{Nd}}$ Verhältnisse, als die östlicheren Einheiten, abgesehen vom Pringles Metamorphic Complex, der ein bimodales Liefergebiet oder Liefergebiete $\mathrm{zu}$ haben scheint. Obwohl die Sierras de Chepes all die gerade aufgezählten Merkmale besitzt, kann nicht ausgeschlossen werden, dass es sich bei dieser Einheit um ein Äquivalent der Puncoviscana Formation, der Sierras de Córdoba und des Conlara Metamorphic Complex handelt, da die gebänderten Gneise in den Sierras de Chepes absolut identisch mit denen des Conlara Metamorphic Complex sind. 
Das Fehlen eines Maximums mit Grenvillian Altern im Nogolí Metamorphic Complex, dem Pringles Metamorphic Complex und der San Luis Formation ist der Hauptunterschied dieser Einheiten zu den anderen (Meta)Sedimenten der östlichen Sierras Pampeanas. Der Nogolí Metamorphic Complex und der Pringles Metamorphic Complex könnten aus Material der pampeanen Orogenese oder dem Dom Feliciano-Gariep Orogen aufgebaut sein. Die Provenienz der San Luis Formation scheint stark auf ein früh- (570 Ma) oder spätpampeanes (517 Ma) Ereignis eingegrenzt zu sein. 


\section{Literaturverzeichnis}

Aceñolaza, F.G., Toselli, A.J. 1981. Geología del Noroeste Argentino. Publicaciones Especiales de la Facultad de las Ciencas Naturalis, Uniersidad Nacional de Tucumán 1287: $1-212$.

Aceñolaza, G.F., Tortello, M.F. 2003. El Alisal: A new locality with trace fossils of the Puncoviscana Formation (Late Precambrian-Early Cambrian) in Salta province, Argentina. Geologica Acta 1: 95-102.

Aceñolaza, F.G., Aceñolaza, G.F. 2005. La Formación Puncoviscana y unidades estratigráficas vinculadas en el Neoproterozoico - Cámbrico Temprano delNoroeste Argentino. Latin American Journal of Sedimentology and Basin Analysis 12 (2): 67-91.

Aceñolaza, F.G., Miller, H., Toselli, A.J. 1983. La geología de la Sierra de Ancasti. Münstersche Forschungen zur Geologie und Paläontologie 59: 1-372.

Aceñolaza, F.G., Miller, H., Toselli, A.J. 1988. The Puncoviscan Formation (Late Precambrian-Early Cambrian). Sedimentology, tectonometamorphic history and age of the oldest rocks of NW Argentina. In: Bahlburg, H., Breitkreuz, C., Giese, P. (Eds.) The Southern Central Andes: Contribution to Structure and Evolution of an Active Continental Margin. Lecture Notes in Earth Sciences 17: 25-38.

Aceñolaza, F.G., Miller, H., Toselli, A.J. 1990. El Ciclo Pampeano en el Noroeste Argentino. Universidad Nacional de Tucumán. Serie Correlacion Geologica 4: 1-227.

Aceñolaza, F.G., Miller, H., Toselli, A.J. 2000. The Pampean and Famatinian cycles superimposed orogenic events in West Gondwana. In: Miller, H., Herve, F. (Eds.) Geoscientific Cooperation with Latin America. Zetschrift für angewandte Geologie, Sonderheft 1: 337-344.

Aceñolaza, F.G., Miller, H., Toselli, A.J. 2002. Proterozoic-Early Paleozoic evolution in western South America - a discussion. Tectonopysics 354:121-137.

Adams, C., Miller, H., Toselli, A.J. 2006. Maximum age and Provenance area of the Puncoviscana Formation Sediments (NW Argentina) based on detrital zircon geochronology - a pilot study- $11^{\text {th }}$ Congresso Geologico Chileno, Actas 1 , Análisis de Cuenca 11-14. 
Adams, C., Miller, H., Toselli, A.J., Griffin, W.L. 2008. The Puncoviscana Formation of northwest Argentina: U-Pb geochronology of detrital zircons and Rb-Sr metamorphic ages and their bearing on its stratigraphic age, sediment provenance and tectonic setting. Neues Jahrbuch für Geologie und Paläontologie - Abhandlungen 247 (3): 341-352.

Aitcheson, S., Harmon, R.S., Moorbath, S., Schneider, A., Soler, P., Soria-Escalante, E., Steele, G., Swainbank, I., Wörner, G. 1995. Pb isotopes define basement domains of the altiplano, central Andes. Geology 23: 555-558.

Armstrong-Altrin, J.S., Verma, S.P. 2005. Critical evaluation of six tectonic setting discrimination diagrams using geochemical data of Neogene sediments from known tectonic settings. Sedimentary Geology 177: 115-129.

Astini, R.A., Benedetto, J.L., Vaccari, N.E. 1995. The early Paleozoic evolution of the Argentine Precordillera as a Laurentian rifted, drifted and collided terrane: A geodynamic model. Geological Society of America Bulletin 107: 253-273.

Bachmann, G., Grauert, B., Kramm, U., Lork, A., Miller, H. 1987. El magmatismo Cámbrico medio Cámbrico superior en el basamento del noroeste Argentino: Investigaciones isotópicas y geocronológicas sobre los granitoides de los complejos intrusivos Santa Rosa de Tastil y Cañani. $10^{\text {th }}$ Congresso Geologico Arggentino 4: 125-127.

Bahlburg, H. 1990. The Ordovician basin in the Puna of NW Argentina and N Chile: Geodynamic evolution from back-arc to foreland basin. Geotektonische Forschungen 75: $1-107$.

Bahlburg, H., 1998. The geochemistry and provenance of Ordovician turbidites in the Puna. In: Pankhurst, R.J., Rapela, C.W. (Eds.) The Proto-Andean Margin of Gondwana. Geological Society of London, Special Publications 142: 127-142.

Baldo, E.G., Demange, M., Martino, R.D. 1996. Evolution of the Sierras de Córdoba, Argentina. Tectonophysics 267: 121-142.

Basei, M.A.S., Siga Jr., O., Masquelin, H., Harara, O.M., Reis Neto, J.M., Preciozzi, F. 2000. The Dom Feliciano belt of Brazil and Uruguay and its foreland domain the Río de la Plata Craton. In: Cordani, U.G., Milani, E.J., Thomaz Filho, A., Campos, D.A. (Eds.) Tectonic Evolution of South America 311-334.

Basei, M.A.S., Frimmel, H.E., Nutman, A.P., Preciozzi, F., Jacob, J. 2005. A connection between the Neoproterozoic Dom Feliciano (Brazil/Uruguay) and Gariep (Namibia/South 
Africa) orogenic belts - evidence from a reconnaissance provenance study. Precambrian Research 139: 195-221.

Bhatia, M.R. 1983. Plate tectonics and geochemical composition of sandstones. The Journal of Geology 91: 611-627.

Bhatia, M.R., Crook, K.A. 1986. Trace element characteristics of greywackes and tectonic setting discrimination of sedimentary basins. Contributions to Mineralogy and Petrology 92: 181-193.

Black, L.P., Kamo, S.L. 2003. TEMORA: a new zircon standard for U-Pb geochronology. Chemical Geology 200: 155-170.

Bock, B., Bahlburg, H., Wörner, G., Zimmermann, U. 2000. Tracing crustal evolution in the southern central Andes from Late Precambrian to Permian with geochemical and Nd and $\mathrm{Pb}$ isotope data. The Journal of Geology 108: 515-535.

Boles, J.R., Franks, S.G. 1979. Clay diagenesis in Wilcox Sandstones of Southwest Texas: implications of smectite diagenesis on sandstone cementation. Journal of Sedimentary Petrology 49: 55-70.

Brito Neves, B.B., Campos Neto, M.C., Fuck, R.A. 1999. From Rodinia to Western Gondwana: An approach to the Brasiliano-Pan African Cycle and orogenic collage. Episodes 22 (3): 155-166.

Brogioni, M. 2001. Geología de los cuerpos Vioroco y El Fierro, faja máfica-ultramáfica del borde oriental de la Sierra de San Luis. Revista de la Asocición Geológica Argentina 56 (3): 281-292.

Casquet, C., Pankhurst, R.J., Galindo, C., Rapela, C.W., Fanning, C.M., Baldo, E., Dahlquist, J., González-Casado, J.M., Colombo, F. 2008. A deformed alkaline igneous rockcarbonatite complex from the Western Sierras Pampeanas, Argentina: Evidence for late Neoproterozoic opening of the Clymene Ocean? Precambrian Research 165 (3-4): 205220.

Cerredo, M.E., López de Luchi, M.G. 2002. Application of provenance and tectonic setting diagrams on metamorphic rocks: The case of metamorphic units from Sierra de San Luis. $6^{\text {th }}$ Congreso de Mineralogía y Metalogenia 77-83, Facultad de las Ciencias Exactas y Naturales, Universidad de Buenos Aires. 
Chappell, B.W., White, J.R. 1992. I- and S-type granites in the Lachlan fold belt. Transactions Royal Society of Edinburgh 83: 1-26.

Chemale Jr., F., Hartmann, L.A., da Silva, L.C. 1995. Stratigraphy and tectonism of the Precambrian to early Paleozoic units of Southern Brazil and Uruguay - excursion guidebook. Acta Geológica Leopoldensia 42: 4-115.

Cingolani, C.A., Hartmann, L.A., Santos, J.O.S., McNaughton, N.J. 2002. U-Pb SHRIMP dating of zircons from the Buenos Aires Complex of the Tandilia Belt, Río de la Plata Craton, Argentina. $15^{\text {th }}$ Congresso Geológico Argentino, Actas 1: 149-154.

Cingolani, C.A., et al. in review. The Argentine Tandilia System as a southern extension of the Río de la Plata craton: An overview. Submitted to: International Journal of Earth Sciences.

Collo, G., Astini, G.A., Cawood, P.A., Buchan, C., Pimentel, M. 2009. U-Pb detrital zircon ages and Sm-Nd isotopic features in low-grade metasedimentary rocks of the Famatina belt: implications for late Neoproterozoic early Palaeozoic evolution of the proto-Andean margin of Gondwana. Journal of the Geological Society 166: 303-319.

Connelly, J., Thrane, K., Krawiec, A., Garde, A. 2006. Linking the Palaeoproterozoic Nagssugtoqidian and Rinkian orogens through the Disko Bugt region of West Greenland. Journal of the Geological Society of London 163: 319-335.

Cordani, U.G., Sato, K., Teixeira, W., Tassinari, C.C.G., Basei, M.A.S. 2000. Crustal evolution of the South American platform. In: Cordani, U.G., Milani, E.J., Thomaz-Filho, A., Campos, D.A. (Eds.) Tectonic Evolution of South America. $31^{\text {st }}$ International Geological Congress, Río de Janeiro, 19-40.

Dahlquist, J.A., Rapela, C.W., Pankhurst, R.J., Baldo, E., Saavedra, J., Alasino, P.H. 2005. Los granitoides de la Sierra de Chepes y su comparación con granitoides paleozoicos de las Sierras Pampeanas: implicancias para el orógeno famatiniano. In: Dahlquist, J.A., Baldo, E.G., Alasino, P.H. (Eds.) Geología de la provincia de La Rioja - PrecámbricoPaleozoico Inferior-. Asociación Geológica Argentina, Serie D, Publicaciones Especiales 8: $87-108$.

Da Silva, L.C., Gresse, P.G., Scheepers, R., McNaughton, N.J., Hartmann, L.A., Fletcher, I. 2000. U-Pb SHRIMP and Sm-Nd age constraints on the timing and sources of the PanAfrican Cape Granite Suite, South Africa. Journal of African Earth Sciences 30 (4): 795815. 
Delpino, S.H., Dimieri, L., Bjerg, E.H., Kostadinoff, J., Mogessie, A., Hoinkes, G., Hauzenberger, C., Felfernig, A. 2001. Geometric analysis and timing of structures on mafic-ultramafic bodies and high-grade metamorphic rocks in the Sierras de San Luis province, Argentina. Journal of South American Earth Sciences 14 (1): 101-112.

Delpino, S.H., Bjerg, E.A., Ferracutti, G.R., Mogessie, A. 2007. Counterclockwise tectonometamorphic evolution of the Pringles Metamorphic Complex, Sierras Pampeanas of San Luis (Argentina). Journal of South American Earth Sciences 23: 147-175.

DePaolo, D.J., Wasserburg, G.J. 1976. Nd isotopic variations and petrogenetic models. Geophysical Research Letters 3: 249-252.

Dickinson, W.R., Suczek, C.A. 1979. Plate tectonics and sandstone compositions. American Association of Petroleum Geologists 63 (12): 2164-2182.

Do Campo, M., Ribeiro Guevara, S. 2002. Geoquímica de las secuencias clásticas de la Formación Puncoviscana (Neoproterozoico, NO Argentina), proveniencia y marco tectónico. $15^{\text {th }}$ Congresso Geológico Argentino, Actas CD-ROM, trabajo 370.

Drobe, M., López de Luchi, M.G., Steenken, A., Frei, R., Naumann, R., Siegesmund, S., Wemmer, K. 2009. Provenance of the late Proterozoic to early Cambrian metaclastic sediments of the Sierra de San Luis (Eastern Sierras Pampeanas) and Cordillera Oriental, Argentina. Journal of South American Earth Sciences 28: 239-262.

Drobe, M., López de Luchi, M.G., Steenken, A., Wemmer, K., Naumann, R., Frei, R., Siegesmund, S. in review. Geodynamic Evolution of the Eastern Sierras Pampeanas based on geochemical, $\mathrm{Sm}-\mathrm{Nd}, \mathrm{Pb}-\mathrm{Pb}$ and SHRIMP data. Submitted to International Journal of Earth Sciences.

Durand, F.R. 1996. La transición Precámbrico-Cámbrico en el sur de Sudamérica. In: Baldis, B., Aceñolaza, F.G. (Eds.) Early Paleozoic evolution in Northwest Gondwana. Universidad Nacionál de Tucumán, Serie Correlation Geológica 12: 195-206.

Eglington, B.M. 2006. Evolution of the Namaqua-Natal belt, southern Africa - a geochronological and isotope geochemical review. Journal of African Earth Sciences 46: 93-111.

Escayola, M.P., Ramé, G., Kraemer, P.E. 1996. Caracterización y significado tectónico de las fajas ultramáficas de las Sierras Pampeans de Córdoba. $13^{\text {th }}$ Congresso Geológico Argentino, Actas 3: 421-438. 
Escayola, M.P., Pimentel, M.M., Armstrong, R. 2007. Neoproterozoic backarc basin: Sensitive high-resolution ion microprobe $\mathrm{U}-\mathrm{Pb}$ and $\mathrm{Sm}-\mathrm{Nd}$ isotopic evidence from the Eastern Pampean Ranges, Argentina. Geological Society of America 35 (6): 495-498.

Finney, S.C., Gleason, J.D., Gehrels, G.G., Peralta, S.H., Aceñolaza, F.G. 2003. Early Gondwanian Connection for the Argentine Precordillera Terrane. Earth and Planetary Science Letters 205: 349-359.

Floyd, P.A., Leveridge, B.E. 1987. Tectonic environment of the Devonian Gramscatho basin, south Cornwall; Framework mode and geochemical evidence from turbidite sandstones. Journal of the Geological Society 144: 531-532.

Frimmel, H.E., Hartnady, C.J.H., Koller, F. 1996. Geochemistry and tectonic setting of magmatic units in the Pan-African Gariep belt, Namibia. Chemical Geology 130: 101121.

Frei, R., Bridgwater, D., Rosing, M., Stecher, O. 1999. Controversial Pb-Pb and Sm-Nd isotope results in the early Archean Isua (West Greenland) oxide iron formation: Preservation of primary signatures versus secondary disturbances. Geochimica et Cosmochimica Acta 63 (3-4): 473-488.

Gebauer, D., Grünenfelder, M. 1976. U-Pb zircon and Rb-Sr whole rock dating of low-grade metasediments. Example: Montagne Noire (Southern France). Contributions to Mineralogy and Petrology 59: 13-32.

Goldstein, S.L., O’Nions, R.K., Hamilton, P.J. 1984. A Sm-Nd isotopic study of atmospheric dusts and particulates from major river systems. Earth and Planetary Science Letters 70: 221-236.

González, P.D., Sato, A.M., Basei, M.A.S., Vlach, S., Llambías, E. 2002. Structure, metamorphism and age of the Pampean-Famatinian orogenies in the western Sierra de San Luis. In: Cabaleri, N., Linares, E., López de Luchi, M.G., Ostera, H. Panarello, H. (Eds.), $15^{\text {th }}$ Congresso Geológico Argentino, Actas 11: 51-56.

Gromet, L.P., Otamendi, J.E., Miró, R.C., Demichelis, A.H., Schwartz, J.J., Tibaldi, A.M. 2005. The Pampean orogeny: ridge subduction or continental collision? Gondwana 12 conference, Mendoza, Academia Nacional de Ciencias, 185.

Grandstaff, D.E., Edelman, M.J., Foster, R.W., Zbinden, E., Kimberly, M.M. 1986. Chemistry and mineralogy of Precambrian paleosols at the base of the Dominion and Pongola Groups, Transvaal, South Africa. Precambrian Research 32: 97-131. 
Harnois, L. 1988. The CIW index: a new chemical index of weathering. Sedimentary Geology 55: 319-322.

Hartmann, L.A., Santos, J.O.S., Cingolani, C.A., McNaughton, N.J. 2002. Two Palaeoproterozoic orogenies in the evolution of the Tandilia Belt, Buenos Aires, as evidenced by zircon U-Pb SHRIMP geochronology. International Geology Review 44: 528-543.

Hauzenberger, C., Mogessie, A., Hoinkes, G., Felfernig, A., Bjerg, E., Kostadinoff, J., Delpino, S.H, Dimieri, L. 2001. Metamorphic evolution of the Sierras de San Luis, Argentina: Granulite facies metamorphism related to mafic intrusions. Mineralogy and Petrology 71 (1-2): 95-126.

Heinrich, H., Herrmann, A.G. 1990. Praktikum der Analytischen Geochemie 1-669. (Springer)

Herron, M.M. 1988. Geochemical classification of terrigenous sands and shales from core or log data. Journal of Sedimentary Petrology 58: 820-829.

Hongn, F.D., Tubía, J.M., Aranguren, A., Mon, R. 2001. El batolito de Tastil (Salta, Argentina): un caso de magmatismo poliorogénico en el basamento andino. Boletín Geológico y Minero 112 (3): 113-124.

Iacumín, M., Piccirillo, E.M., Girardi, V.A.V., Teixeira, W., Bellieni, G., Etcheveste, H., Fernández, R., Pinese, J.P.P., Ribot, A. 2001. Early Proterozoic calc-alkaline and Middle Proterozoic tholeiitic dyke swarms from central-eastern Argentina: petrology, geochemistry, Sr-Nd isotopes and tectonic implications. Journal of Petrology 42: 21092143.

Jahn, B.M., Condie, K.C. 1995. Evolution of the Kaapvaal Craton as viewed from geochemical and $\mathrm{Sm}-\mathrm{Nd}$ isotopic analyses of cratonic pelites. Geochimica et Cosmochimica Acta 59: 2239-2258.

Janasi, V.A., Leite, R.J., van Schmus, W.R. 2001. U-Pb chronostratigraphy of the granitic magmatism in the Agudos Grandes Batholith (west of Sao Paulo, Brazil) - implications for the evolution of the Ribeira belt. Journal of South American Earth Sciences 14: 363376.

Ježek, P., Miller, H. 1986. Deposition and fades distribution of turbiditic sediments of the Puncoviscana Formation (Upper Precambrian-Lower Cambrian) within the basement of 
the NW Argentine Andes. Zentralblatt für Geologie und Paläontologie 1 (9-10): 12351244.

Kay, S., Orrell, S., Abbruzzi, J.M. 1996. Zircon and whole rock Nd-Pb isotopic evidence for a Grenville Age and a Laurentian origin for the basement of the Precordillera in Argentina. The Journal of Geology 104: 637-648.

Keppie, J.D., Bahlburg, H. 1999. Puncoviscana Formation northwestern and central Argentina: Passive margin or foreland basin deposit? Geological Society of America, Special Paper 336: 139-143.

Kilmurray, J.O., Dalla Salda, L. 1977. Caracteres estructurales y petrológicos de la region central y sur de la Sierra de San Luis. Obra del Centenario del Museo de La Plata 4 :167178.

Kraemer, P.E., Escayola, M.P., Martino, R.D. 1995. Hipótesis sobre la evolución neoproterozoica de las Sierras Pampeanas de Córdoba (3084’-3284’S) Argentina. Revista de la Asociación Geológica Argentina 50 (1-4): 47-59.

Krogstad, E.J., Fedo, C.M., Eriksson, K.A. 2004. Provenance ages and alteration histories of shales from the Middle Archean Buhwa greenstone belt, Zimbabwe: Nd and Pb isotopic evidence. Geochimica et Cosmochimica Acta. 68 (2): 319-332.

Leal, P.R., Hartmann, L.A., Santos, J.O.S., Miró, R.C., Ramos, V.A. 2003. Volcanismo postorogénico en el extremo norte de las Sierras Pampeanas Orientales: Nuevos datos geocronológicos y sus implicancias tectónicas. Revista de la Asociación Geológica Argentina 58 (4):593-607.

Leite, J.A.D., Hartmann, L.A., Fernandes, L.A.D., McNaughton, N.J., Soliani Jr., E., Koester, E., Santos, J.O.S., Vasconcellos, J.O.S., Vasconcellos, M.A.Z. 2000. Zircon U-Pb SHRIMP dating of the Dom Feliciano belt, southernmost Brazil. Journal of South American Earth Sciences 13: 739-750.

Lira, R., Millone, H.A., Kirschbaum, A.M., Moreno, R.S. 1997. Calcalkaline arc granitoid activity in the Sierra Norte-Ambargasta Ranges, central Argentina. Journal of South American Earth Sciences 10: 157-177.

Llambías, E.J., Sato, A.M., Ortiz Suárez, A., Prozzi, C. 1998. The granitoids of the Sierra de San Luis. In: Pankhurst, R.J., Rapela, C.W. (Eds.), The Proto-Andean Margin of Gondwana. Geological Society of London, Special Publication 142: 325-341. 
Llambías, E.J., Gregori, D., Basei, M.A.S., Varela, R., Prozzi, C. 2003. Ignimbritas riolíticas neoproterozoicas en la Sierra Norte de Córdoba: ¿evidencia de un arco magmático temprano en el ciclo Pampeano? Revista de la Asociación Geológica Argentina 58 (4): $572-582$.

Loewy, S.L., Connelly, J.N., Dalziel, I.W.D., Gower, C.F. 2003. Eastern Laurentia in Rodinia: Constraints from whole-rock $\mathrm{Pb}$ and $\mathrm{U}-\mathrm{Pb}$ geochronology. In: Sircombe, K.N., McElhinny, M.W. (Eds.) Orogenic belts, regional and global tectonics: A memorial volume to Chris McAulay Powell. Tectonophysics 375: 169-197.

Loewy, S.L., Connelly, J.N., Dalziel, I.W.D. 2004. An orphaned basement block: The Arequipa-Antofalla Basement of the central Andean margin of South America. Geological Society of America Bulletin 116: 171-187.

López de Luchi, M.G. 1986. Geología y Petrología del basamento de la Sierra de San Luis, Región del Batolito de Renca. Ph.D. Thesis, Departamento de Geología de la Facultad de Ciencias Exactas, Universidad de Buenos Aires 1-374.

López de Luchi, M.G. 1987. Caracterización geológica y geoquímica del Plutón La Tapera y del Batolito de Renca, Sierra de San Luis. $10^{\text {th }}$ Congresso Geológico Argentino, Actas 4: 84-87.

López de Luchi, M.G. 1996. Enclaves en un batolito postectónico: Petrología de los enclaves microgranulares del batolito de Renca. Revista de la Asociación Geológica Argentina 51 (2): 131-146.

López de Luchi, M.G., Cerredo, M.E., Rossello, E. 1999. Protoliths of a metasedimentary sequence: A case study on the Lower Paleozoic Pacific margin of Gondwana. Annual Meeting of the Geological Society of America. Abstracts with Programs 31 (7): A298.

López de Luchi, M.G., Cerredo, M.E., Siegesmund, S., Steenken, A., Wemmer, K. 2003. Provenance and tectonic setting of the protoliths of the Metamorphic Complexes of Sierra de San Luis. Revísta de la Asociación Geológica Argentina 58 (4): 525-540.

López de Luchi, M.G., Rapalini, A.E., Siegesmund, S., Steenken, A. 2004. Application of magnetic fabrics to the emplacement and tectonic history of Devonian granitoids in Central Argentina. In: Martín-Hernández, F., Luneburg, C., Aubourg, C., Jackson, M. (Eds.) Magnetic Fabric: Methods and applications. Geological Society of London, Special Publication 238: 447-474. 
López de Luchi, M.G., Siegesmund, S., Wemmer, K., Steenken, A., Naumann, R. 2008. Geochemical constraints on the petrogenesis of the Palaeozoic granitoids of the Sierra de San Luis, Sierras Pampeanas, Argentina. Journal of South American Earth Sciences 24 (24): $138-166$.

Lork, A., Miller, H., Kramm, U. 1990. U-Pb zircon and monazite ages of the Angostura granite and the orogenic history of the northwest Argentine Basement. Journal of South American Earth Sciences 2: 147-153.

Lucassen, F., Becchio, R., Wilke, H.G., Franz, G., Thirlwall, M.F., Viramonte, J., Wemmer, K. 2000. Proterozoic-Paleozoic development of the basement of the Central Andes (18$26^{\circ}$ S) - a mobile belt of the South American craton. Journal of South American Earth Sciences 13: 697-715.

Lucassen, F., Becchio, R., Harmon, R., Kasemann, S., Franz, G., Trumbull, R., Wilke, H.G., Romer, R.L., Dulski, P. 2001. Composition and density model of the continental crust at an active continental margin-the Central Andes between $21^{\circ}$ and $27^{\circ} \mathrm{S}$. Tectonophysics 341: 195-223.

Lucassen, F., Harmon, R., Franz, G., Romer, R.L., Becchio, R., Siebel, W. 2002. Lead evolution of the Pre-Mesozoic crust in the Central Andes (18-27): progressive homogenisation of Pb. Chemical Geology 186: 183-197.

Ludwig, K.R. 2000. SQUID 1.00, A User’s Manual. Berkeley Geochronology Center, Special Publications 2.

Ludwig, K.R. 2003. Isoplot/Ex Version 3.00: a Geochronological Toolkit for Microsoft Excel. Berkeley Geochronology Center, Special Publications 4.

Martino, R.D. 2003 Las fajas de deformación dúctil de las Sierras Pampeanas de Córdoba: una reseña general. Revista de la Asociación Geológica Argentina 58 (4): 549-571.

McLennan, S.M. 1989. Rare earth elements in sedimentary rocks: Influence of provenance and sedimentary process. Mineralogica Society of America, Reviews in Mineralogy 21: 169-200.

McLennan, S.M. 2001. Relationships between the trace element composition of sedimentary rocks and upper continental crust. Geochemistry Geophysics Geosystems 2: $2000 G C 000109$.

McLennan, S.M., Taylor, S.R. 1991. Sedimentary rocks and crustal evolution: Tectonic setting and secular trends. The Journal of Geology 99 (1): 1-21. 
McLennan, S.M., Taylor, S.R., McCulloch, M.T., Maynard, J.B. 1990. Geochemical and NdSr isotopic composition of deep-sea turbidites: Crustal evolution and plate tectonic associations. Geochimica et Cosmochimica Acta 54: 2015-2050.

McLennan, S.M, Hemming, S., McDaniel, D., Hanson, G. 1993. Geochemical approaches to sedimentation, provenance and tectonics. Geological Society of America, Special Paper 284: $21-40$.

Mutti, D. 1987. Estudio geológico del complejo Gabro-Peridotítico del área de Bosque Alegre, provincia de Córdoba. PhD. Thesis, Facultad de Ciencias Exactas y Naturales, Universidad de Buenos Aires 1-398.

Nance, W.B., Taylor, S.R. 1976. Rare earth element patterns and crustal evolution I. Australian post-Archean rocks. Geochimica et Cosmochimica Acta 40: 1539-1551.

Nesbit, H.W., Young, G.M. 1982. Early proterozoic climates and plate motions inferred from major element chemistry of lutites. Nature 299: 715-717.

Nesbit, H.W., Markovics, G., Price, R.C. 1980. Prediction of some weathering trends of plutonic and volcanic rocks based on thermodynamic and kinetic considerations. Geochimica et Cosmochimica Acta 44: 1659-1666.

Nesbit, H.W., Young, G.M., McLennan, S.M., Keays, R.R. 1996. Effects of chemical weathering and sorting on the petrogenesis of siliclastic sediments with implications for provenance studies. The Journal of Geology 104: 525-542.

Omarini, R.H. 1983. Caracterización litológia, diferenciación y genesis de la Formación Puncoviscana entre el Valle de Lerma y la Faja Eruptiva de la Puna. PhD. Thesis, Universidad Nacionál de Salta, Argentina 1-202.

Omarini, R.H., Sureda, R.J., Götze, H.J., Seilacher, A., Pflüger, F. 1999. Puncoviscana folded belt in northwestern Argentina: Testimony of late Proterozoic Rodinia fragmentation and pre-Gondwana collisional episodes. International Journal of Earth Sciences 88: 76-97.

Ortiz Suárez, A., Prozzi, C.R., Llambías, E.J. 1992. Geología de la parte sur de la Sierra de San Luis y granitoides asociados, Argentina. Estudios Geológicos 48: 269-277.

Pankhurst, R.J., Rapela, C.W. 1998. In: Pankhurst, R.J., Rapela, C.W. (Eds.) The ProtoAndean margin of Gondwana. Geological Society of London, Special Publication 142: 1383. 
Pankhurst, R.J., Ramos, A., Linares, E. 2003. Antiquity and evolution of the Río de la Plata craton in Tandilia, southern Buenos Aires province, Argentina. Journal of South American Earth Sciences 16: 5-13.

Paces, J.B., Miller, J.D. 1993. Precise U-Pb ages of Duluth Complex and related mafic intrusions, Northeastern Minnesota: geochronological insights to physical, petrogenic, paleomagnetic and tectonomagmatic processes associated with the $1.1 \mathrm{Ga}$ midcontinent rift system. Journal of Geophysical Research 98 (B): 13,997-14,013.

Preciozzi, F., Peel, E., Muzio, R., Ledesma, J.J., Guerequiz, R. 2001. Dom Feliciano belt and Punta del Este Terrane: Geochronological features. In: $3^{\text {rd }}$ South American Symposium on Isotope Geology, Pucón, Chile, 217-220.

Prozzi, C.R., Ramos, G. 1988. La Formación San Luis. $1^{\text {st }}$ Jornadas de trabajo de Sierras Pampeanas, San Luis Abstracts 1.

Prozzi, C.R., Zimmermann, U. 2005. Provenance of metasedmientary successions of the sierra de San Luis: first results. $16^{\text {th }}$ Congresso Geológico Argentino.

Ramos, V.A. 1988. Late Proterozoic-Early Paleozoic of South America: A collisional story. Episodes 11: 168-174.

Ramos, V.A. 2008. The basement of the Central Andes: The Arequipa and related Terranes. Annual review of Earth and Planetary Sciences 36: 289-324.

Ramos, V.A., Jordan, T.E., Allmendinger, W., Mpodozis, C., Kay, S.M., Cortés, J.M., Palma, M. 1986. Paleozoic terranes of the Central Argentine-Chilean Andes. Tectonics 5: 855880.

Ramos, V.A., Cristallini, E.O., Pérez, D.J. 2002. The Pampean flat-slab of the Central Andes. Journal of South American Earth Sciences 15: 59-78.

Rapela, C.W. 2000. The Sierras Pampeanas of Argentina: Paleozoic building of the southern Proto-Andes. In: Cordani, U.G., Milani, E.J., Thomas-Filho, A., Campos, D.A. (Eds.) Tectonic Evolution of South America, $31^{\text {st }}$ International Geological Congress, Río de Janeiro 381-387.

Rapela, C.W., Toselli, A.J., Heaman, L., Saavedra, J. 1990. Granite plutonism of the Sierras Pampeanas: An inner cordilleran Paleozoic arc in the southern Andes. Geological Society of America, Special Paper 241: 77-90. 
Rapela, C.W., Pankhurst, R.J., Casquet, C., Baldo, E., Saavedra, J., Galindo, C., Fanning, C.M. 1998. The Pampean Orogeny of the southern proto-Andes: Cambrian continental collision in the Sierras de Córdoba. In: Pankhurst, R.J., Rapela, C.W. (Eds.) The ProtoAndean Margin of Gondwana Geological Society of London, Special Publications 142: $181-217$.

Rapela, C.W., Pankhurst, R.J., Casquet, C., Fanning, C.M., Baldo, E.G., Gonzáles-Casado, J.M., Galindo, C., Dahlquist, J. 2007. The Río de la Plata craton and the assembly of Gondwana. Earth-Science Reviews 83: 49-82.

Roser, B.P., Cooper, R.A., Nathan, S., Tulloch, A.J. 1996. Reconnaissance sandstone geochemistry, provenance and tectonic setting of the lower Paleozoic terranes of the West Coast and Nelson, New Zealand. New Zealand Journal of Geology and Geophysics 39: 116.

Rossi de Toselli, J.N., Durand, F.R., Toselli, A.J., Sardi, F.G. 1997. Aspectos estratigráficos y geocímicos comparatives del basamento metamórfico de bajo grado del Sistema de Famatina, Argentina. Revista de la Asociación Geológica Argentina 52: 469-480.

Rozendaal, A., Gresse, P.G., Scheepers, R., Le Roux, J.P. 1999. Neoproterozoic to Early Cambrian crustal evolution of the Pan-African Saldania belt, South Africa. Precambrian Research 97: 303-323.

Sato, A.M., González, P.D. Sato, K. 2001. First indication of Mesoproterozoic age from the western basement of the Sierra de San Luis, Argentina, $3^{\text {rd }}$ South American Symposium on Isotope Geology, Extended Abstracts (CD edition), Sociedad Geológica de Chile, 6467.

Sato, A.M., Gonzáles, P.D., Basei, M.A.S., Llambias, E.J. 2006. U-Pb ages of Komatiitic Rocks from Sierra de San Luis, Argentina, $5^{\text {th }}$ South American Symposium on Isotope Geology, Short Papers, 169-173.

Scheepers, R., Armstrong, R. 2002. New U-Pb SHRIMP zircon ages of the Cape Granite Suite: Implications for the magmatic evolution of the Saldania Belt. South African Journal of Geology 105: 241-256.

Schwartz, J.J., Gromet, L.P. 2004. Provenance of a Late Proterozoic-Early Cambrian basin, Sierras de Córdoba, Argentina. Precambrian Research 129: 1-21. 
Schwartz, J.J., Gromet, L.P., Miró, R. 2008. Timing and Duration of the Calc-Alkaline Arc of the Pampean Orogeny: Implications for the Late Neoproterozoic to Cambrian Evolution of Western Gondwana. The Journal of Geology 116: 39-61.

Siegesmund, S., Steenken, A., López de Luchi, M.G., Wemmer, K., Hoffmann, A., Mosch, S. 2004. The Las Chacras-Potrerillos Batholith: Structural Evidences on its Emplacement and Timing of the Intrusion. International Journal of Earth Sciences 93: 23-43.

Siegesmund, S., Steenken, A., Martino, R.D., Wemmer, K., López de Luchi, M.G., Frei, R., Presnyakow, S., Guerschi, A. 2009. Time constraints on the tectonic Evolution of the Eastern Sierras Pampeanas (Central Argentina). International Journal of Earth Sciences: doi:10.1007/s00531-009-0471-z.

Sims, J.P., Skirrow, R.G., Stuart-Smith, P.G., Lyons, P. 1997. Informe geológico y metalogenético de las Sierras de San Luis y Comechingones (provincias de San Luis y Córdoba), 1:250000. Anales 28, IGRM, SEGEMAR, Buenos Aires, 1-148.

Sims, J.P., Ireland, T.R., Cmacho, A., Lyons, P., Pieters, P.E., Skirrow, R.G., Stuart-Smith, P.G., Miró, R. 1998. U-Pb, Th-Pb and Ar-Ar geochronology form the southern Sierras Pampeanas: Implication for the Palaeozoic tectonic evolution of the western Gondwana margin. In: Pankhurst, R.J., Rapela, C.W. (Eds.) The Proto-Andean Margin of Gondwana. Geological Society of London, Special Publications 142: 259-281.

Söllner, F., Brodtkorb, M.K. de, Miller, H., Pezzutti, N.E., Fernández, R.R. 2000. U-Pb zircon ages of metavolcanic rocks from the Sierra de San Luis, Argentina. Revista de la Asociación Geológica Argentina 55 (1-2): 15-22.

Steenken, A., López de Luchi, M.G., Siegesmund, S., Wemmer, K., Pawlig, S. 2004. Crustal provenance and cooling of the basement complexes of the Sierra de San Luis: An insight into the tectonic history of the proto-Andean margin of Gondwana. Gondwana Research $\mathbf{7}$ (4): 1171-1195.

Steenken, A., Siegesmund, S., López de Luchi, M.G., Frei, R., Wemmer, K. 2006. Neoproterozoic to Early Palaeozoic events in the Sierra de San Luis: Implications for the Famatinian geodynamics in the Eastern Sierras Pampeanas (Argentina). Journal of the Geological Society of London 163: 965-982.

Steenken, A., López de Luchi, M.G., Siegesmund, S., Wemmer, K., Naumann, R. 2007. Geochemical constraints on the petrogenesis of the Paleozoic granitoids of the Sierra de 
San Luis, Sierras Pampeanas, Argentina. Journal of South American Earth Sciences 24: 138-166.

Steenken, A., Siegesmund, S., Wemmer, K., López de Luchi, M.G. 2008. Time constraints on the Famatinian and Achalian structural evolution of the basement of the Sierra de San Luis (Eastern Sierras Pampeanas, Argentina), Journal of South American Earth Sciences 25 (3): 336-358.

Stuart-Smith, P.G., Camacho, A., Sims, J.P., Skirrow, R.G., Lyons, P., Pieters, P.E., Black, L.P. 1999. Uranium-lead dating of felsic magmatic cycles in the southern Sierras Pampeanas, Argentina: Implications for the tectonic development of the proto-Andean Gondwana margin. In: Ramos, V.A., Keppie, J.D. (Eds.) Laurentia-Gondwana Connections before Pangea. Geological Society of America, Special Paper 336: 87-114.

Taylor, S.R., McLennan, S.M. 1985. The Continental Crust: Its Composition and Evolution. Blackwell Science, Oxford Evolution, 1-312. (Blackwell Science)

Thomas, W.A., Astini, R.A. 2003. Ordovician accretion of the Argentine Precordillera terrane to Gondwana: A review. Journal of South American Earth Sciences 16: 67-79.

Thomas, W.A., Astini, R.A., Bayona, G. 2002. Ordovician collision of the Argentine Precordillera with Gondwana, independent of Laurentian Taconic Orogeny. Tectonophysics 345: 131-152.

Trindade, R.I.F., D’Agrella-Filho, M.S., Epof, I., Brito Neves, B.B. 2006. Paleomagnetism of early Cambrian Itabaiana mafic dikes (NE Brazil) and the final assembly of Gondwana. Earth and Planetary Science Letters 244: 361-377.

Todt, W., Cliff, R.A., Hanser, A., Hofmann, A.W. 1993. Re-calibration of NBS lead standards using a ${ }^{202} \mathrm{~Pb}+{ }^{205} \mathrm{~Pb}$ double spike. Terra Abstracts 5: Supplement 1.

Toselli, A.J. 1990. Metamorfismo del Ciclo Pampeano. In: Aceñolaza, F.G., Miller, H., Toselli, A.J. (Eds.) El Ciclo Pampeano en el Noroeste Argentino. Universidad Nacional de Tucumán, Argentina. Serie Correlación Geológica 4: 181-197.

Toselli, A.J., Aceñolaza, F.G. 1978. Geocronología de las Formaciones Puncoviscana y Suncho, Provincias de Salta y Catamarca. Revista de la Asociación Geológica Argentina 33: 76-80. 
Toselli, A.J., 1990. Metamorfismo del Ciclo Pampeano. In: Aceñolaza FG, Miller H, Toselli AJ (Eds.) El Ciclo Pampeano en el Noroeste Argentino.INSUGEO, Serie de Correlación Geológica 4: 181-197.

Tosdal, R.M. 1996. The Amazon-Laurentian connection as viewed from the Middle Proterozoic rocks in the central Andes, western Bolivia and northern Chile. Tectonics 15: 827-842.

Turner, J.C.M. 1960. Estratigrafía de la Sierra de Santa Victoria y adyacencias. - Boletín de la Academia Nacional de Ciencias en Córdoba 41 (2): 163-169.

von Eynatten, H., Barceló-Vidal, C., Pawlowsky-Glahn, V. 2003. Composition and discrimination of sandstones: A stochastic evaluation of different analytical methods. Journal of Sedimentary Research 73: 47-57.

von Gosen, W., Prozzi, C. 1998. Structural evolution of the Sierra de San Luis (Eastern Sierras Pampeanas, Argentina): Implications for the Proto-Andean margin of Gondwana. In: Pankhurst, R.J., Rapela, C.W. (Eds.) The Proto-Andean Margin of Gondwana. Geological Society of London, Special Publication 142: 235-258.

Wemmer, K., Steenken, A., Müller, S., López de Luchi, M.G., Siegesmund, S. in review. The tectonic significance of K/Ar Illite fine-fraction ages from the San Luis Formation (Eastern Sierras Pampeanas). Submitted to International Journal of Earth Sciences.

Williams, I.S. 1998. U-Th-Pb geochronology by ion microprobe. In: McKibben, M.A., Shanks, W.C.P. III, Ridley, W.I. (Eds.) Applications of Microanalytical Techniques to Understanding Mineralizing Processes. Reviews in Economic Geology 7: 1-35.

Willner, A.P. 1990. División tectonometamórfica del basamento del noroeste Argentino. In: Aceñolaza, F.G., Miller, H., Toselli, A.J. (Eds.) El Ciclo Pampeano en el Noroeste Argentino. Universidad Nacionál de Tucumán, Argentina. Serie Correlation Geológica 4: 113-159.

Willner, A.P., Miller, H., Ježek, P. 1985. Geochemical features of an Upper PrecambrianLower Cambrian greywacke/pelite sequence (Puncoviscana trough) from the basement of the NW-Argentine Andes. Neues Jahrbuch für Geologie und Paläontologie, Mitteilungen 33: 498-512.

Zimmermann, U. 1999. Sedimentpetrographische, geochemische und isotopengeochemische Methoden zur Bestimmung der Beziehung von Provenienz und Ablagerungungsraum an 
aktiven Kontinentalrändern: Das ordovizische back-arc Becken in der Süd-Puna, Hochland im Nordwesten Argentiniens. PhD thesis, University of Heidelberg, Germany, $1-282$.

Zimmermann, U., Bahlburg, H. 2003. Provenance analyses and paleotectonic setting of the clastic Ordovician deposits in the southern Puna, NW Argentina. Sedimentology 50: 1079-1104.

Zimmermann, U. 2005. Provenance studies of very low to low-grade metasedimentary rocks of the Puncoviscana complex, northwest Argentina. In: Vaughan, A.P.M., Leat, P.T., Pankhurst, R.J. (Eds.) Terrane processes at the margin of Gondwana. Geological Society of London, Special Publication 246: 381-416. 
Anhang 1: Übersicht über die Haupt- und Spurenelemente, den normierten Werten der REE (Taylor and McLennan 1985), sowie einiger ausgewählter Spurenelementverhältnisse aller untersuchter Proben aus den östlichen Sierras Pampeanas und der Cordillera Oriental.

San Luis Formation

\begin{tabular}{|c|c|c|c|c|c|c|c|c|c|}
\hline Probe & & A7-01 & A9-01 & A11-01 & A17-01 & A30-01 & A32-01 & A34-01 & A12-06 \\
\hline $\mathrm{SiO}_{2}$ & Gew. \% & 64.21 & 76.22 & 56.77 & 63.49 & 62.24 & 61.51 & 55.70 & 81.90 \\
\hline $\mathrm{TiO}_{2}$ & Gew. \% & 0.87 & 0.52 & 0.84 & 0.66 & 0.80 & 0.74 & 0.85 & 0.34 \\
\hline $\mathrm{Al}_{2} \mathrm{O}_{3}$ & Gew. \% & 17.52 & 11.50 & 21.02 & 16.34 & 17.50 & 17.82 & 20.57 & 9.41 \\
\hline $\mathrm{Fe}_{2} \mathrm{O}_{3}$ & Gew. \% & 5.42 & 3.90 & 6.27 & 5.31 & 6.54 & 6.74 & 7.80 & 2.03 \\
\hline $\mathrm{MnO}$ & Gew. \% & 0.03 & 0.05 & 0.06 & 0.09 & 0.16 & 0.21 & 0.04 & 0.02 \\
\hline $\mathrm{MgO}$ & Gew. \% & 2.43 & 0.85 & 2.22 & 1.87 & 2.28 & 2.64 & 2.43 & 0.67 \\
\hline $\mathrm{CaO}$ & Gew. \% & 0.33 & 0.25 & 0.17 & 0.43 & 0.57 & 0.34 & 0.29 & 0.13 \\
\hline $\mathrm{Na}_{2} \mathrm{O}$ & Gew. \% & 0.81 & 1.67 & 0.37 & 0.99 & 1.78 & 1.57 & 0.45 & 1.34 \\
\hline $\mathrm{K}_{2} \mathrm{O}$ & Gew. \% & 4.76 & 2.05 & 5.89 & 4.14 & 4.15 & 4.43 & 5.22 & 2.41 \\
\hline $\mathrm{P}_{2} \mathrm{O}_{5}$ & Gew. \% & 0.15 & 0.11 & 0.05 & 0.08 & 0.11 & 0.12 & 0.10 & 0.04 \\
\hline $\mathrm{H}_{2} \mathrm{O}$ & Gew. \% & 3.03 & 2.42 & 5.45 & 4.62 & 3.20 & 3.52 & 5.60 & 1.21 \\
\hline $\mathrm{CO}_{2}$ & Gew. \% & 0.09 & 0.28 & 0.52 & 1.61 & 0.39 & 0.13 & 0.69 & 0.16 \\
\hline Summe & Gew. \% & 99.65 & 99.81 & 99.63 & 99.82 & 99.73 & 99.77 & 99.74 & 99.65 \\
\hline $\mathrm{Li}$ & ppm & 45 & 35 & 42 & 43 & 18 & 63 & 55 & 58 \\
\hline $\mathrm{Be}$ & $\mathrm{ppm}$ & 2.5 & 2.1 & 4.1 & 2.3 & 1.5 & 3.8 & 3.8 & 4.0 \\
\hline $\mathrm{Sc}$ & $\mathrm{ppm}$ & 10.9 & 7.0 & 8.1 & 9.9 & 3.7 & 9.8 & 12.2 & 8.0 \\
\hline V & $\mathrm{ppm}$ & 100 & 67 & 156 & 199 & 90 & 111 & 133 & 44 \\
\hline $\mathrm{Cr}$ & $\mathrm{ppm}$ & 88 & 56 & 99 & 79 & 78 & 86 & 104 & $<10$ \\
\hline Co & $\mathrm{ppm}$ & 12 & 9 & 10 & 6 & 5 & 15 & 7 & 3 \\
\hline $\mathrm{Ni}$ & $\mathrm{ppm}$ & 28 & 24 & 46 & 42 & 40 & 47 & 37 & 6 \\
\hline $\mathrm{Cu}$ & $\mathrm{ppm}$ & 21 & 20 & 52 & 29 & 13 & 32 & 34 & 13 \\
\hline $\mathrm{Zn}$ & $\mathrm{ppm}$ & 92 & 59 & 87 & 66 & 39 & 115 & 102 & 58 \\
\hline$Y$ & $\mathrm{ppm}$ & 17 & 15 & 22 & 19 & 16 & 18 & 23 & 25 \\
\hline $\mathrm{Zr}$ & $\mathrm{ppm}$ & 149 & 139 & 159 & 128 & 140 & 152 & 159 & 215 \\
\hline $\mathrm{Nb}$ & $\mathrm{ppm}$ & 18 & 13 & 16 & 15 & 9 & 17 & 18 & 17 \\
\hline Mo & $\mathrm{ppm}$ & 0.6 & 0.3 & 1.3 & 2.8 & 1.0 & 0.2 & 0.7 & 0.5 \\
\hline $\mathrm{Cd}$ & $\mathrm{ppm}$ & 0.2 & 0.1 & 0.2 & 0.2 & 0.1 & 0.0 & 0.0 & 0.0 \\
\hline Sn & $\mathrm{ppm}$ & 4.1 & 3.0 & 4.0 & 4.0 & 2.3 & 5.4 & 4.4 & 4.0 \\
\hline $\mathrm{Sb}$ & $\mathrm{ppm}$ & 0.1 & 0.7 & 1.2 & 0.3 & 0.3 & 2.1 & 1.7 & 0.3 \\
\hline $\mathrm{Rb}$ & $\mathrm{ppm}$ & 118 & 93 & 138 & 113 & 73 & 126 & 112 & 188 \\
\hline $\mathrm{Sr}$ & $\mathrm{ppm}$ & 90 & 106 & 46 & 64 & 83 & 47 & 58 & 89 \\
\hline Cs & $\mathrm{ppm}$ & 17.7 & 5.2 & 12.1 & 8.5 & 5.5 & 15.5 & 6.8 & 13.0 \\
\hline $\mathrm{Ba}$ & $\mathrm{ppm}$ & 694 & 301 & 350 & 358 & 177 & 361 & 579 & 420 \\
\hline La & $\mathrm{ppm}$ & 21 & 18 & 28 & 19 & 21 & 22 & 28 & 39 \\
\hline $\mathrm{Ce}$ & $\mathrm{ppm}$ & 41 & 43 & 55 & 40 & 34 & 41 & 57 & 84 \\
\hline $\operatorname{Pr}$ & $\mathrm{ppm}$ & 5.5 & 4.8 & 7.5 & 5.2 & 5.0 & 6.0 & 7.8 & 11.0 \\
\hline $\mathrm{Nd}$ & $\mathrm{ppm}$ & 20.9 & 18.2 & 28.3 & 19.4 & 19.0 & 22.9 & 30.0 & 38.6 \\
\hline $\mathrm{Sm}$ & $\mathrm{ppm}$ & 4.0 & 3.7 & 5.6 & 3.9 & 3.8 & 4.7 & 5.9 & 7.6 \\
\hline $\mathrm{Eu}$ & $\mathrm{ppm}$ & 0.8 & 0.7 & 1.0 & 0.7 & 0.7 & 0.8 & 1.1 & 1.0 \\
\hline Gd & $\mathrm{ppm}$ & 3.3 & 3.1 & 4.9 & 3.2 & 3.2 & 4.2 & 5.3 & 6.1 \\
\hline Tb & $\mathrm{ppm}$ & 0.6 & 0.5 & 0.7 & 0.6 & 0.6 & 0.6 & 0.8 & 0.9 \\
\hline Dy & $\mathrm{ppm}$ & 3.2 & 3.1 & 4.5 & 3.5 & 3.1 & 3.9 & 4.9 & 5.2 \\
\hline Ho & $\mathrm{ppm}$ & 0.7 & 0.6 & 0.9 & 0.7 & 0.6 & 0.8 & 1.0 & 1.0 \\
\hline $\mathrm{Er}$ & $\mathrm{ppm}$ & 2.1 & 1.9 & 2.7 & 2.3 & 1.8 & 2.4 & 3.0 & 2.9 \\
\hline $\mathrm{Tm}$ & $\mathrm{ppm}$ & 0.3 & 0.3 & 0.4 & 0.3 & 0.3 & 0.4 & 0.4 & 0.4 \\
\hline $\mathrm{Yb}$ & $\mathrm{ppm}$ & 2.3 & 1.9 & 2.7 & 2.3 & 1.8 & 2.5 & 2.9 & 3.1 \\
\hline Lu & $\mathrm{ppm}$ & 0.3 & 0.3 & 0.4 & 0.3 & 0.3 & 0.4 & 0.4 & 0.5 \\
\hline $\mathrm{Hf}$ & $\mathrm{ppm}$ & 4.8 & 4.4 & 4.5 & 3.4 & 4.6 & 4.2 & 4.2 & 7.0 \\
\hline $\mathrm{Ta}$ & $\mathrm{ppm}$ & 1.3 & 0.9 & 0.7 & 1.1 & 0.7 & 1.0 & 1.1 & 1.5 \\
\hline W & $\mathrm{ppm}$ & 3.5 & 2.3 & 1.6 & 4.0 & 1.1 & 2.4 & 3.0 & 2.0 \\
\hline $\mathrm{Ti}$ & $\mathrm{ppm}$ & 1.0 & 0.6 & 1.4 & 0.9 & 0.4 & 1.2 & 1.0 & 1.0 \\
\hline $\mathrm{Pb}$ & $\mathrm{ppm}$ & 12.8 & 6.1 & 7.8 & 14.2 & 16.8 & 18.4 & 9.5 & 33.0 \\
\hline $\mathrm{Bi}$ & $\mathrm{ppm}$ & 0.1 & 0.2 & 0.6 & 0.6 & 0.3 & 0.9 & 0.2 & 0.6 \\
\hline Th & $\mathrm{ppm}$ & 11.3 & 8.0 & 10.4 & 10.2 & 6.4 & 8.6 & 11.8 & 21.2 \\
\hline$u$ & $\mathrm{ppm}$ & 2.4 & 2.1 & 2.4 & 2.7 & 1.4 & 1.0 & 2.6 & 5.6 \\
\hline \multicolumn{10}{|c|}{ Chondrit normierte Werte (Taylor und McLennan 1985) } \\
\hline La & & 56.8 & 49.3 & 75.8 & 50.7 & 56.5 & 58.8 & 75.3 & 107.0 \\
\hline $\mathrm{Ce}$ & & 43.1 & 44.5 & 57.2 & 42.2 & 35.8 & 43.3 & 60.1 & 87.5 \\
\hline $\operatorname{Pr}$ & & 40.4 & 35.3 & 54.8 & 37.7 & 36.6 & 43.7 & 57.0 & 80.3 \\
\hline $\mathrm{Nd}$ & & 29.4 & 25.6 & 39.8 & 27.3 & 26.7 & 32.2 & 42.2 & 54.3 \\
\hline Sm & & 17.5 & 15.8 & 24.2 & 16.8 & 16.3 & 20.3 & 25.7 & 32.9 \\
\hline Eu & & 9.4 & 8.0 & 11.0 & 8.2 & 8.1 & 9.1 & 12.8 & 11.5 \\
\hline Gd & & 10.9 & 10.1 & 16.1 & 10.5 & 10.6 & 13.7 & 17.3 & 19.8 \\
\hline Tb & & 9.6 & 9.3 & 12.9 & 9.9 & 9.5 & 11.1 & 13.8 & 15.4 \\
\hline Dy & & 8.4 & 8.0 & 11.8 & 9.1 & 8.0 & 10.2 & 12.8 & 13.6 \\
\hline Ho & & 7.9 & 7.3 & 11.0 & 8.7 & 7.2 & 9.4 & 11.8 & 12.2 \\
\hline Er & & 8.6 & 7.5 & 11.0 & 9.2 & 7.4 & 9.7 & 11.9 & 11.7 \\
\hline $\mathrm{Tm}$ & & 8.9 & 7.4 & 11.2 & 9.2 & 7.1 & 9.9 & 11.9 & 12.3 \\
\hline $\mathrm{Yb}$ & & 9.2 & 7.6 & 11.0 & 9.3 & 7.2 & 10.1 & 11.6 & 12.6 \\
\hline Lu & & 9.1 & 7.3 & 10.4 & 9.0 & 7.1 & 10.1 & 11.5 & 12.9 \\
\hline $\mathrm{Cr} N \mathrm{~N}$ & & 0.88 & 0.84 & 0.63 & 0.40 & 0.87 & 0.78 & 0.78 & \\
\hline $\mathrm{Th} / \mathrm{Sc}$ & & 1.04 & 1.14 & 1.28 & 1.03 & 1.74 & 0.88 & 0.97 & 2.65 \\
\hline $\mathrm{La} / \mathrm{Th}$ & & 1.8 & 2.3 & 2.7 & 1.8 & 3.2 & 2.5 & 2.3 & 1.9 \\
\hline $\mathrm{Ti} / \mathrm{Zr}$ & & 58.4 & 37.1 & 53.0 & 51.6 & 57.4 & 49.1 & 53.8 & 15.6 \\
\hline $\mathrm{La} / \mathrm{Sc}$ & & 1.9 & 2.6 & 3.4 & 1.9 & 5.6 & 2.2 & 2.3 & 4.9 \\
\hline $\mathrm{Cr} / \mathrm{Ni}$ & & 3.1 & 2.3 & 2.2 & 1.9 & 2.0 & 1.8 & 2.8 & \\
\hline $\mathrm{Y} / \mathrm{Ni}$ & & 0.62 & 0.63 & 0.47 & 0.45 & 0.41 & 0.39 & 0.62 & 4.21 \\
\hline $\mathrm{Zr} / \mathrm{Sc}$ & & 13.7 & 19.9 & 19.6 & 12.8 & 37.8 & 15.5 & 13.0 & 27.0 \\
\hline $\mathrm{Nb} / \mathrm{Y}$ & & 1.0 & 0.8 & 0.7 & 0.8 & 0.6 & 1.0 & 0.8 & 0.7 \\
\hline Th/U & & 4.8 & 3.9 & 4.4 & 3.9 & 4.4 & 8.7 & 4.5 & 3.8 \\
\hline $\mathrm{La}, / \mathrm{Sm}_{w}$ & & 3.2 & 3.1 & 3.1 & 3.0 & 3.5 & 2.9 & 2.9 & 3.3 \\
\hline $\mathrm{La}_{N} / \mathrm{Yb}_{N}$ & & 6.2 & 6.5 & 6.9 & 5.5 & 7.8 & 5.8 & 6.5 & 8.5 \\
\hline $\mathrm{Eu} / \mathrm{Eu}^{*}$ & & 0.68 & 0.63 & 0.56 & 0.62 & 0.62 & 0.55 & 0.61 & 0.45 \\
\hline $\mathrm{Ce} / \mathrm{Ce}^{*}$ & & 0.96 & 1.14 & 0.95 & 1.04 & 0.83 & 0.91 & 0.98 & 1.04 \\
\hline ELREE & $\mathrm{ppm}$ & 93 & 87 & 124 & 87 & 83 & 97 & 129 & 131 \\
\hline ¿REE & $\mathrm{ppm}$ & 106 & 100 & 142 & 101 & 95 & 113 & 149 & 201 \\
\hline
\end{tabular}


Nogolí Metamorphic Complex

Pringles Metamorphic Complex

\begin{tabular}{|c|c|c|c|c|c|c|c|c|c|c|c|}
\hline \multicolumn{5}{|c|}{ Nogolı Metamorphic complex } & \multicolumn{7}{|c|}{ Pringles IMetamorphic complex } \\
\hline Probe & A39-01 & A07-06 & A08-06 & A16-06 & Probe & A05-01 & A13-06 & A14-06 & A15-06 & A18-06 & A19-06 \\
\hline $\mathrm{SiO}_{2}$ & 54.88 & 75.65 & 79.13 & 75.31 & $\mathrm{SiO} 2$ & 70.48 & 87.59 & 72.09 & 59.38 & 79.64 & 84.39 \\
\hline $\mathrm{TiO}_{2}$ & 1.01 & 0.51 & 0.46 & 0.57 & $\mathrm{TiO} 2$ & 0.78 & 0.27 & 0.51 & 1.04 & 0.46 & 0.69 \\
\hline $\mathrm{Al}_{2} \mathrm{O}_{3}$ & 20.21 & 11.51 & 10.01 & 11.55 & $\mathrm{Al} 2 \mathrm{O} 3$ & 12.97 & 6.21 & 13.82 & 18.46 & 10.69 & 7.37 \\
\hline $\mathrm{Fe}_{2} \mathrm{O}_{3}$ & 9.32 & 3.26 & 2.75 & 3.59 & $\mathrm{Fe} 2 \mathrm{O} 3$ & 5.24 & 1.05 & 3.98 & 8.90 & 2.21 & 1.69 \\
\hline $\mathrm{MnO}$ & 0.14 & 0.08 & 0.06 & 0.04 & $\mathrm{MnO}$ & 0.10 & 0.08 & 0.07 & 0.13 & 0.03 & 0.03 \\
\hline $\mathrm{MgO}$ & 2.85 & 1.28 & 1.19 & 1.50 & $\mathrm{MgO}$ & 2.02 & 0.33 & 1.37 & 3.39 & 0.90 & 0.58 \\
\hline $\mathrm{CaO}$ & 1.28 & 2.15 & 1.28 & 0.66 & $\mathrm{CaO}$ & 1.69 & 1.38 & 0.39 & 0.44 & 0.24 & 1.35 \\
\hline $\mathrm{Na}_{2} \mathrm{O}$ & 2.73 & 2.97 & 2.09 & 1.74 & $\mathrm{Na} 2 \mathrm{O}$ & 2.23 & 1.31 & 1.05 & 0.86 & 0.20 & 2.02 \\
\hline $\mathrm{K}_{2} \mathrm{O}$ & 5.24 & 1.51 & 1.80 & 3.41 & $\mathrm{~K} 2 \mathrm{O}$ & 3.22 & 0.58 & 4.85 & 4.95 & 3.27 & 0.71 \\
\hline $\mathrm{P}_{2} \mathrm{O}_{5}$ & 0.18 & 0.17 & 0.13 & 0.12 & P2O5 & 0.08 & 0.06 & 0.09 & 0.11 & 0.04 & 0.20 \\
\hline $\mathrm{H}_{2} \mathrm{O}$ & 1.86 & 0.68 & 0.95 & 1.25 & $\mathrm{H} 2 \mathrm{O}$ & 0.75 & 0.62 & 1.54 & 1.86 & 1.70 & 0.66 \\
\hline $\mathrm{CO}_{2}$ & 0.03 & 0.03 & 0.03 & 0.03 & $\mathrm{CO} 2$ & 0.16 & 0.41 & 0.03 & 0.12 & 0.30 & 0.07 \\
\hline Summe & 99.73 & 99.81 & 99.88 & 99.77 & Summe & 99.72 & 99.89 & 99.78 & 99.63 & 99.69 & 99.77 \\
\hline $\mathrm{Li}$ & 53 & 31 & 28 & 80 & $\mathrm{Li}$ & 15 & 13 & 70 & 53 & 48 & 16 \\
\hline $\mathrm{Be}$ & 3.5 & 1.5 & 1.0 & 0.2 & $\mathrm{Be}$ & 0.9 & 2.1 & 1.3 & 2.0 & 1.6 & 2.0 \\
\hline $\mathrm{Sc}$ & 14.8 & 9.7 & 8.6 & 6.7 & $\mathrm{Sc}$ & 12.3 & 4.9 & 11.2 & 20.0 & 7.5 & 3.4 \\
\hline V & 118 & 68 & 52 & 79 & V & 88 & 35 & 73 & 158 & 51 & 35 \\
\hline $\mathrm{Cr}$ & 105 & 43 & 36 & 47 & $\mathrm{Cr}$ & 71 & 18 & 49 & 105 & 36 & 38 \\
\hline Co & 19 & 8 & 8 & 10 & Co & 11 & 4 & 10 & 24 & 3 & 3 \\
\hline $\mathrm{Ni}$ & 40 & 17 & 13 & 18 & $\mathrm{Ni}$ & 21 & 5 & 20 & 54 & 6 & 8 \\
\hline $\mathrm{Cu}$ & 11 & 33 & 7 & 6 & $\mathrm{Cu}$ & 12 & 7 & 4 & 11 & 9 & 14 \\
\hline $\mathrm{Zn}$ & 151 & 64 & 59 & 82 & $\mathrm{Zn}$ & 94 & 36 & 72 & 164 & 52 & 42 \\
\hline $\mathrm{Y}$ & 33 & 31 & 25 & 19 & $Y$ & 37 & 23 & 22 & 32 & 28 & 21 \\
\hline $\mathrm{Zr}$ & 205 & 174 & 160 & 217 & $\mathrm{Zr}$ & 228 & 273 & 123 & 213 & 231 & 759 \\
\hline $\mathrm{Nb}$ & 23 & 14 & 11 & 13 & $\mathrm{Nb}$ & 16 & 18 & 14 & 21 & 15 & 14 \\
\hline Mo & 0.2 & 0.1 & 0.2 & 0.1 & Mo & 0.2 & 0.3 & 0.2 & 0.2 & 0.3 & 0.1 \\
\hline $\mathrm{Cd}$ & 0.0 & 0.0 & 0.0 & 0.0 & $\mathrm{Cd}$ & 0.1 & 0.1 & 0.0 & 0.0 & 0.0 & 0.1 \\
\hline Sn & 5.0 & 3.2 & 3.1 & 0.8 & Sn & 0.7 & 2.5 & 4.3 & 4.1 & 1.8 & 0.8 \\
\hline $\mathrm{Sb}$ & 0.2 & 0.2 & 0.3 & 0.1 & $\mathrm{Sb}$ & 0.0 & 0.4 & 0.2 & 0.0 & 0.1 & 0.4 \\
\hline $\mathrm{Rb}$ & 144 & 130 & 97 & 65 & $\mathrm{Rb}$ & 129 & 68 & 273 & 187 & 166 & 45 \\
\hline $\mathrm{Sr}$ & 120 & 112 & 110 & 87 & $\mathrm{Sr}$ & 117 & 170 & 63 & 99 & 68 & 132 \\
\hline Cs & 9.9 & 7.3 & 3.9 & 3.4 & Cs & 2.1 & 4.0 & 13.7 & 8.7 & 9.7 & 3.5 \\
\hline $\mathrm{Ba}$ & 534 & 135 & 234 & 585 & $\mathrm{Ba}$ & 536 & 263 & 586 & 611 & 487 & 158 \\
\hline La & 37 & 40 & 37 & 22 & La & 46 & 31 & 41 & 55 & 34 & 29 \\
\hline $\mathrm{Ce}$ & 78 & 81 & 76 & 51 & $\mathrm{Ce}$ & 98 & 77 & 91 & 116 & 73 & 68 \\
\hline $\mathrm{Pr}$ & 9.1 & 9.8 & 9.0 & 6.7 & $\mathrm{Pr}$ & 12.2 & 8.3 & 10.5 & 14.1 & 8.6 & 6.9 \\
\hline $\mathrm{Nd}$ & 33.3 & 35.6 & 34.0 & 25.3 & $\mathrm{Nd}$ & 46.4 & 29.4 & 38.3 & 53.8 & 31.3 & 25.0 \\
\hline $\mathrm{Sm}$ & 6.5 & 7.0 & 6.6 & 5.1 & $\mathrm{Sm}$ & 9.3 & 5.9 & 7.4 & 10.7 & 6.0 & 4.6 \\
\hline $\mathrm{Eu}$ & 1.3 & 1.2 & 1.1 & 0.9 & Eu & 1.4 & 0.9 & 1.2 & 1.8 & 0.9 & 0.6 \\
\hline Gd & 6.3 & 6.8 & 6.1 & 4.4 & $\mathrm{Gd}$ & 8.3 & 5.1 & 6.3 & 9.5 & 5.1 & 4.0 \\
\hline $\mathrm{Tb}$ & 1.0 & 1.0 & 0.8 & 0.7 & $\mathrm{~Tb}$ & 1.2 & 0.8 & 0.9 & 1.3 & 0.8 & 0.6 \\
\hline Dy & 6.5 & 6.1 & 5.1 & 3.9 & Dy & 7.2 & 4.8 & 4.7 & 7.0 & 4.6 & 3.7 \\
\hline Ho & 1.4 & 1.2 & 1.0 & 0.8 & Ho & 1.5 & 1.0 & 0.9 & 1.3 & 0.9 & 0.8 \\
\hline Er & 4.3 & 3.4 & 2.7 & 2.3 & Er & 4.2 & 3.1 & 2.4 & 3.4 & 2.6 & 2.4 \\
\hline $\mathrm{Tm}$ & 0.6 & 0.5 & 0.4 & 0.3 & $\mathrm{Tm}$ & 0.6 & 0.5 & 0.3 & 0.5 & 0.4 & 0.4 \\
\hline$Y b$ & 4.2 & 3.2 & 2.6 & 2.4 & $\mathrm{Yb}$ & 4.1 & 3.4 & 2.2 & 3.1 & 2.7 & 2.6 \\
\hline Lu & 0.6 & 0.5 & 0.4 & 0.4 & Lu & 0.6 & 0.5 & 0.3 & 0.5 & 0.4 & 0.4 \\
\hline $\mathrm{Hf}$ & 5.5 & 5.1 & 4.8 & 5.9 & $\mathrm{Hf}$ & 5.8 & 9.1 & 3.7 & 3.4 & 6.3 & 20.0 \\
\hline $\mathrm{Ta}$ & 1.5 & 1.2 & 0.8 & 0.8 & $\mathrm{Ta}$ & 0.8 & 1.8 & 1.2 & 1.2 & 1.1 & 1.0 \\
\hline W & 1.3 & 0.6 & 0.5 & 0.3 & W & 0.1 & 0.9 & 4.4 & 0.5 & 2.2 & 1.0 \\
\hline $\mathrm{Ti}$ & 1.3 & 0.8 & 0.6 & 0.6 & $\mathrm{Ti}$ & 0.9 & 0.4 & 1.4 & 1.3 & 0.7 & 0.3 \\
\hline $\mathrm{Pb}$ & 19.9 & 14.0 & 17.0 & 21.6 & $\mathrm{~Pb}$ & 22.6 & 26.1 & 17.7 & 26.1 & 13.0 & 11.9 \\
\hline $\mathrm{Bi}$ & 0.2 & 0.1 & 0.1 & 0.1 & $\mathrm{Bi}$ & 0.0 & 0.1 & 0.1 & 0.1 & 0.2 & 0.4 \\
\hline Th & 15.8 & 13.1 & 13.5 & 10.2 & Th & 17.2 & 33.0 & 15.2 & 22.2 & 16.0 & 17.3 \\
\hline U & 2.8 & 3.1 & 2.4 & 1.4 & U & 2.4 & 5.4 & 2.8 & 2.7 & 3.7 & 5.3 \\
\hline
\end{tabular}

Chondrit normierte Werte (Taylor und McLennan 1985)

\begin{tabular}{|c|c|c|c|c|c|c|c|c|c|c|c|}
\hline \multicolumn{4}{|c|}{ it normierte Werte (Taylor und McLennan 1985) } & \multicolumn{5}{|c|}{ Chondrit normierte Werte (Taylor und McLennan 1985) } & \multirow[b]{2}{*}{150.5} & \multirow[b]{2}{*}{92.6} & \multirow{2}{*}{78.0} \\
\hline La & 101.3 & 108.7 & 99.8 & 60.8 & La & 125.0 & 84.4 & 112.8 & & & \\
\hline $\mathrm{Ce}$ & 82.0 & 84.9 & 79.0 & 53.7 & $\mathrm{Ce}$ & 102.1 & 80.5 & 94.7 & 121.0 & 76.6 & 71.1 \\
\hline $\mathrm{Pr}$ & 66.2 & 71.2 & 66.0 & 48.9 & $\mathrm{Pr}$ & 88.9 & 60.3 & 76.4 & 102.7 & 62.7 & 50.2 \\
\hline $\mathrm{Nd}$ & 46.8 & 50.1 & 47.8 & 35.6 & $\mathrm{Nd}$ & 65.3 & 41.4 & 53.9 & 75.6 & 44.0 & 35.2 \\
\hline $\mathrm{Sm}$ & 28.0 & 30.3 & 28.5 & 22.2 & $\mathrm{Sm}$ & 40.3 & 25.4 & 32.1 & 46.3 & 26.2 & 20.0 \\
\hline Eu & 14.6 & 13.7 & 12.9 & 10.0 & Eu & 15.6 & 10.0 & 13.4 & 20.9 & 10.5 & 7.4 \\
\hline Gd & 20.6 & 22.3 & 20.1 & 14.3 & Gd & 27.0 & 16.6 & 20.7 & 31.1 & 16.7 & 13.2 \\
\hline Tb & 17.5 & 17.4 & 14.6 & 11.3 & Tb & 20.6 & 13.8 & 14.8 & 21.6 & 13.7 & 10.2 \\
\hline Dy & 17.0 & 15.9 & 13.3 & 10.4 & Dy & 18.8 & 12.6 & 12.4 & 18.4 & 12.0 & 9.6 \\
\hline $\mathrm{Ho}$ & 16.4 & 14.4 & 11.7 & 9.5 & Ho & 17.2 & 11.8 & 10.3 & 15.4 & 10.9 & 9.3 \\
\hline Er & 17.3 & 13.5 & 10.9 & 9.4 & $\mathrm{Er}$ & 17.0 & 12.6 & 9.6 & 13.8 & 10.6 & 9.7 \\
\hline $\mathrm{Tm}$ & 17.5 & 13.9 & 11.0 & 9.6 & $\mathrm{Tm}$ & 16.9 & 13.5 & 9.8 & 13.2 & 11.0 & 10.5 \\
\hline $\mathrm{Yb}$ & 17.0 & 12.8 & 10.4 & 9.6 & $\mathrm{Yb}$ & 16.6 & 13.6 & 9.0 & 12.6 & 10.8 & 10.6 \\
\hline Lu & 16.5 & 12.5 & 10.5 & 9.5 & Lu & 15.9 & 13.9 & 8.9 & 12.0 & 10.5 & 11.4 \\
\hline $\mathrm{Cr} / \mathrm{N}$ & 0.89 & 0.63 & 0.69 & 0.59 & $\mathrm{Cr} / \mathrm{N}$ & 0.80 & 0.51 & 0.67 & 0.67 & 0.70 & 1.08 \\
\hline $\mathrm{Th} / \mathrm{Sc}$ & 1.07 & 1.35 & 1.57 & 1.52 & $\mathrm{Th} / \mathrm{Sc}$ & 1.4 & 6.7 & 1.4 & 1.1 & 2.1 & 5. \\
\hline $\mathrm{La} / \mathrm{Th}$ & 2.4 & 3.0 & 2.7 & 2.2 & $\mathrm{La} / \mathrm{Th}$ & 2.7 & 0.9 & 2.7 & 2.5 & 2.1 & 1.7 \\
\hline $\mathrm{Ti} / \mathrm{Zr}$ & 49.1 & 29.5 & 28.6 & 26.3 & $\mathrm{Ti} / \mathrm{Zr}$ & 34.2 & 9.8 & 40.9 & 48.6 & 20.1 & 9.1 \\
\hline $\mathrm{La} / \mathrm{Sc}$ & 2.5 & 4.1 & 4.3 & 3.3 & $\mathrm{La} / \mathrm{Sc}$ & 3.7 & 6.3 & 3.7 & 2.8 & 4.5 & 8.4 \\
\hline $\mathrm{Cr} / \mathrm{Ni}$ & 2.6 & 2.5 & 2.7 & 2.6 & $\mathrm{Cr} / \mathrm{Ni}$ & 3.4 & 3.5 & 2.4 & 2.0 & 6.2 & 4.7 \\
\hline $\mathrm{Y} / \mathrm{Ni}$ & 0.8 & 1.8 & 1.9 & 1.0 & $\mathrm{Y} / \mathrm{Ni}$ & 1.7 & 4.5 & 1.1 & 0.6 & 4.8 & 2.6 \\
\hline $\mathrm{Zr} / \mathrm{Sc}$ & 13.8 & 17.9 & 18.6 & 32.3 & $\mathrm{Zr} / \mathrm{Sc}$ & 18.6 & 55.4 & 11.0 & 10.6 & 30.8 & 223.3 \\
\hline $\mathrm{Nb} / \mathrm{Y}$ & 0.7 & 0.5 & 0.4 & 0.7 & $\mathrm{Nb} / \mathrm{Y}$ & 0.4 & 0.8 & 0.7 & 0.7 & 0.5 & 0.7 \\
\hline Th/U & 5.6 & 4.2 & 5.7 & 7.2 & $\mathrm{Th} / \mathrm{U}$ & 7.3 & 6.1 & 5.4 & 8.2 & 4.3 & 3.3 \\
\hline $\mathrm{La}_{N} / \mathrm{Sm}_{\mathrm{N}}$ & 3.6 & 3.6 & 3.5 & 2.7 & $\mathrm{La}_{N} / \mathrm{Sm}_{w}$ & 3.1 & 3.3 & 3.5 & 3.3 & 3.5 & 3.9 \\
\hline $\mathrm{La}_{N} / \mathrm{Yb}_{*}$ & 6.0 & 8.5 & 9.6 & 6.3 & $\mathrm{La}_{N} / \mathrm{Yb}_{N}$ & 7.5 & 6.2 & 12.5 & 12.0 & 8.6 & 7.3 \\
\hline $\mathrm{Eu} / \mathrm{Eu}^{*}$ & 0.61 & 0.53 & 0.54 & 0.56 & $\mathrm{Eu} / \mathrm{Eu}^{*}$ & 0.47 & 0.49 & 0.52 & 0.55 & 0.50 & 0.46 \\
\hline $\mathrm{Ce} / \mathrm{Ce}^{\star}$ & 1.06 & 1.03 & 1.03 & 1.07 & $\mathrm{Ce} / \mathrm{Ce}^{*}$ & 1.03 & 1.23 & 1.09 & 1.03 & 1.08 & 1.21 \\
\hline$\sum$ LREE & 164 & 174 & 162 & 111 & $\Sigma$ LREE & 211 & 152 & 188 & 250 & 153 & 133 \\
\hline$\sum$ REE & 191 & 197 & 182 & 127 & ¿REE & 240 & 172 & 207 & 278 & 172 & 149 \\
\hline
\end{tabular}


Conlara Metamorphic Complex

\begin{tabular}{|c|c|c|c|c|c|c|}
\hline \multicolumn{7}{|c|}{ IIC COI } \\
\hline Probe & A21-01 & A23-01 & A61-05 & A73-05 & A09-06 & A10-06 \\
\hline $\mathrm{SiO}_{2}$ & 70.26 & 68.22 & 70.22 & 74.17 & 68.09 & 72.66 \\
\hline $\mathrm{TiO}_{2}$ & 0.78 & 0.70 & 0.81 & 0.69 & 0.74 & 0.96 \\
\hline $\mathrm{Al}_{2} \mathrm{O}_{3}$ & 13.08 & 13.37 & 12.53 & 11.31 & 14.03 & 11.56 \\
\hline $\mathrm{Fe}_{2} \mathrm{O}_{3}$ & 5.13 & 5.08 & 4.75 & 4.10 & 5.32 & 4.82 \\
\hline $\mathrm{MnO}$ & 0.08 & 0.08 & 0.09 & 0.07 & 0.09 & 0.09 \\
\hline $\mathrm{MgO}$ & 2.16 & 2.24 & 2.25 & 1.68 & 2.57 & 1.86 \\
\hline $\mathrm{CaO}$ & 1.63 & 1.87 & 2.14 & 2.16 & 2.04 & 2.32 \\
\hline $\mathrm{Na}_{2} \mathrm{O}$ & 2.01 & 2.63 & 2.54 & 2.51 & 2.89 & 2.05 \\
\hline $\mathrm{K}_{2} \mathrm{O}$ & 2.79 & 2.55 & 2.45 & 1.65 & 2.49 & 1.96 \\
\hline $\mathrm{P}_{2} \mathrm{O}_{5}$ & 0.20 & 0.18 & 0.23 & 0.21 & 0.19 & 0.28 \\
\hline $\mathrm{H}_{2} \mathrm{O}$ & 1.60 & 2.18 & 1.46 & 1.06 & 1.26 & 1.14 \\
\hline $\mathrm{CO}_{2}$ & 0.04 & 0.68 & 0.28 & 0.07 & 0.04 & 0.04 \\
\hline Summe & 99.77 & 99.78 & 99.75 & 99.69 & 99.75 & 99.73 \\
\hline $\mathrm{Li}$ & 34 & 50 & 49 & 97 & 106 & 53 \\
\hline $\mathrm{Be}$ & 2.0 & 2.9 & 2.1 & 2.5 & 2.8 & 2.0 \\
\hline $\mathrm{Sc}$ & 9.7 & 10.2 & 10.1 & 13.8 & 15.2 & 13.2 \\
\hline V & 102 & 97 & 67 & 75 & 113 & 97 \\
\hline $\mathrm{Cr}$ & 71 & 75 & 58 & 70 & 75 & 74 \\
\hline Co & 11 & 13 & 10 & 15 & 17 & 9 \\
\hline $\mathrm{Ni}$ & 24 & 30 & 27 & 35 & 35 & 20 \\
\hline $\mathrm{Cu}$ & 38 & 16 & 6 & 32 & 35 & 28 \\
\hline $\mathrm{Zn}$ & 83 & 89 & 69 & 49 & 110 & 63 \\
\hline $\mathrm{Y}$ & 29 & 19 & 32 & 35 & 35 & 43 \\
\hline $\mathrm{Zr}$ & 280 & 202 & 277 & 343 & 185 & 338 \\
\hline $\mathrm{Nb}$ & 15 & 14 & 13 & 19 & 16 & 16 \\
\hline Mo & 0.2 & 0.3 & 0.0 & 0.2 & 0.3 & 0.1 \\
\hline $\mathrm{Cd}$ & 0.2 & 0.1 & 0.1 & 0.0 & 0.0 & 0.0 \\
\hline Sn & 3.3 & 2.9 & 1.7 & 1.3 & 1.4 & 3.0 \\
\hline $\mathrm{Sb}$ & 0.0 & 0.2 & 0.0 & 0.1 & 0.1 & 0.2 \\
\hline $\mathrm{Rb}$ & 94 & 100 & 122 & 83 & 163 & 104 \\
\hline $\mathrm{Sr}$ & 106 & 164 & 215 & 217 & 220 & 191 \\
\hline Cs & 6.6 & 9.9 & 13.3 & 24.8 & 27.3 & 6.1 \\
\hline $\mathrm{Ba}$ & 516 & 432 & 506 & 376 & 328 & 372 \\
\hline La & 32 & 38 & 38 & 32 & 35 & 52 \\
\hline $\mathrm{Ce}$ & 67 & 79 & 80 & 71 & 79 & 117 \\
\hline $\mathrm{Pr}$ & 8.7 & 9.8 & 9.9 & 8.4 & 9.3 & 13.5 \\
\hline $\mathrm{Nd}$ & 33.5 & 38.0 & 38.0 & 32.3 & 35.5 & 49.7 \\
\hline $\mathrm{Sm}$ & 6.9 & 7.4 & 7.6 & 6.5 & 7.1 & 9.9 \\
\hline $\mathrm{Eu}$ & 1.3 & 1.3 & 1.4 & 1.3 & 1.4 & 1.7 \\
\hline Gd & 6.5 & 6.3 & 6.7 & 5.7 & 6.2 & 9.6 \\
\hline $\mathrm{Tb}$ & 1.0 & 0.8 & 0.9 & 0.9 & 1.0 & 1.4 \\
\hline Dy & 5.7 & 4.4 & 5.4 & 5.2 & 5.8 & 8.4 \\
\hline Ho & 1.2 & 0.8 & 1.0 & 1.1 & 1.2 & 1.7 \\
\hline $\mathrm{Er}$ & 3.4 & 2.1 & 2.9 & 3.0 & 3.3 & 4.8 \\
\hline $\mathrm{Tm}$ & 0.5 & 0.3 & 0.4 & 0.4 & 0.5 & 0.7 \\
\hline $\mathrm{Yb}$ & 3.1 & 1.9 & 2.7 & 2.9 & 3.2 & 4.5 \\
\hline Lu & 0.5 & 0.3 & 0.4 & 0.4 & 0.5 & 0.7 \\
\hline $\mathrm{Hf}$ & 7.1 & 5.3 & 7.3 & 4.4 & 4.9 & 9.7 \\
\hline $\mathrm{Ta}$ & 0.9 & 1.0 & 0.9 & 1.0 & 1.1 & 1.3 \\
\hline W & 1.9 & 0.7 & 2.3 & 1.6 & 1.8 & 0.7 \\
\hline $\mathrm{Ti}$ & 0.6 & 0.8 & 0.7 & 0.7 & 0.8 & 0.6 \\
\hline $\mathrm{Pb}$ & 19.8 & 28.5 & 18.1 & 20.1 & 22.1 & 8.1 \\
\hline $\mathrm{Bi}$ & 0.2 & 0.1 & 0.1 & 0.1 & 0.1 & 0.1 \\
\hline Th & 10.9 & 12.6 & 14.3 & 13.1 & 11.1 & 18.3 \\
\hline$U$ & 2.3 & 2.6 & 3.3 & 2.8 & 3.1 & 3.5 \\
\hline \multicolumn{7}{|c|}{ Chondrit normierte Werte (Taylor und McLennan 1985) } \\
\hline La & 86.5 & 102.5 & 102.7 & 86.7 & 95.4 & 140.5 \\
\hline $\mathrm{Ce}$ & 70.3 & 83.0 & 83.7 & 74.6 & 82.0 & 122.4 \\
\hline $\operatorname{Pr}$ & 63.2 & 71.6 & 72.2 & 61.4 & 67.6 & 98.4 \\
\hline $\mathrm{Nd}$ & 47.1 & 53.5 & 53.5 & 45.4 & 49.9 & 69.9 \\
\hline Sm & 29.8 & 32.1 & 33.0 & 28.0 & 30.8 & 42.9 \\
\hline $\mathrm{Eu}$ & 14.7 & 15.4 & 15.8 & 14.7 & 16.2 & 19.9 \\
\hline Gd & 21.1 & 20.7 & 21.7 & 18.5 & 20.3 & 31.3 \\
\hline $\mathrm{Tb}$ & 16.5 & 14.3 & 16.1 & 15.4 & 16.9 & 24.5 \\
\hline Dy & 15.0 & 11.5 & 14.1 & 13.7 & 15.1 & 22.0 \\
\hline Ho & 13.7 & 9.4 & 12.2 & 12.5 & 13.8 & 19.9 \\
\hline Er & 13.5 & 8.3 & 11.6 & 12.2 & 13.4 & 19.2 \\
\hline $\mathrm{Tm}$ & 13.3 & 7.9 & 11.3 & 12.3 & 13.5 & 19.2 \\
\hline $\mathrm{Yb}$ & 12.6 & 7.6 & 11.1 & 11.9 & 13.0 & 18.1 \\
\hline Lu & 12.5 & 7.4 & 10.6 & 11.3 & 12.4 & 18.1 \\
\hline $\mathrm{Cr} N \mathrm{~N}$ & 0.70 & 0.78 & 0.86 & 0.93 & 0.66 & 0.77 \\
\hline $\mathrm{Th} / \mathrm{Sc}$ & 1.1 & 1.2 & 1.4 & 0.9 & 0.7 & 1.4 \\
\hline $\mathrm{La} / \mathrm{Th}$ & 2.9 & 3.0 & 2.6 & 2.4 & 3.2 & 2.8 \\
\hline $\mathrm{Ti} / \mathrm{Zr}$ & 28.0 & 34.5 & 29.1 & 20.2 & 40.0 & 28.4 \\
\hline $\mathrm{La} / \mathrm{Sc}$ & 3.3 & 3.7 & 3.7 & 2.3 & 2.3 & 3.9 \\
\hline $\mathrm{Cr} / \mathrm{Ni}$ & 3.0 & 2.5 & 2.1 & 2.0 & 2.2 & 3.7 \\
\hline $\mathrm{Y} / \mathrm{Ni}$ & 1.2 & 0.6 & 1.2 & 1.0 & 1.0 & 2.2 \\
\hline $\mathrm{Zr} / \mathrm{Sc}$ & 29.0 & 19.8 & 27.4 & 24.9 & 12.2 & 25.7 \\
\hline $\mathrm{Nb} / \mathrm{Y}$ & 0.5 & 0.7 & 0.4 & 0.5 & 0.5 & 0.4 \\
\hline $\mathrm{Th} / \mathrm{U}$ & 4.8 & 4.9 & 4.3 & 4.6 & 3.6 & 5.3 \\
\hline $\mathrm{La}_{N} / \mathrm{Sm}_{N}$ & 2.9 & 3.2 & 3.1 & 3.1 & 3.1 & 3.3 \\
\hline $\mathrm{La}_{N} / \mathrm{Yb}_{N}$ & 6.9 & 13.6 & 9.3 & 7.3 & 7.3 & 7.8 \\
\hline $\mathrm{Eu} / \mathrm{Eu}^{*}$ & 0.59 & 0.60 & 0.59 & 0.65 & 0.65 & 0.55 \\
\hline $\mathrm{Ce} / \mathrm{Ce}^{*}$ & 1.01 & 1.02 & 1.03 & 1.03 & 1.08 & 1.12 \\
\hline$\Sigma$ LREE & 148 & 172 & 173 & 173 & 165 & 242 \\
\hline$\Sigma$ REE & 171 & 191 & 195 & 171 & 188 & 275 \\
\hline
\end{tabular}


Puncoviscana Formation

\begin{tabular}{|c|c|c|c|c|c|c|c|c|c|c|c|}
\hline Probe & A85-06 & A86-06 & A87-06 & A88-06 & A89-06 & A90-06 & A91-06 & A93-06 & A94-06 & A96-06 & A97-06 \\
\hline $\mathrm{SiO}_{2}$ & 58.91 & 63.22 & 74.84 & 71.87 & 66.52 & 70.89 & 73.09 & 71.64 & 69.64 & 70.23 & 68.10 \\
\hline $\mathrm{TiO}_{2}$ & 0.83 & 0.80 & 0.45 & 0.59 & 0.77 & 0.51 & 0.54 & 0.55 & 0.48 & 0.85 & 0.82 \\
\hline $\mathrm{Al}_{2} \mathrm{O}_{3}$ & 17.82 & 16.88 & 11.18 & 12.74 & 15.44 & 12.41 & 11.78 & 12.97 & 13.04 & 13.01 & 14.23 \\
\hline $\mathrm{Fe}_{2} \mathrm{O}_{3}$ & 7.95 & 6.41 & 2.99 & 4.17 & 5.15 & 3.38 & 4.75 & 3.73 & 3.93 & 5.00 & 5.46 \\
\hline $\mathrm{MnO}$ & 0.07 & 0.08 & 0.05 & 0.08 & 0.05 & 0.11 & 0.06 & 0.06 & 0.06 & 0.10 & 0.10 \\
\hline $\mathrm{MgO}$ & 3.16 & 2.46 & 1.34 & 1.95 & 2.24 & 1.53 & 2.03 & 1.40 & 1.68 & 2.09 & 2.52 \\
\hline $\mathrm{CaO}$ & 0.43 & 0.50 & 1.37 & 0.62 & 0.36 & 1.62 & 0.47 & 1.29 & 1.95 & 2.29 & 1.68 \\
\hline $\mathrm{Na}_{2} \mathrm{O}$ & 0.05 & 0.63 & 1.63 & 1.33 & 2.37 & 1.52 & 2.19 & 3.11 & 2.29 & 2.75 & 1.94 \\
\hline $\mathrm{K}_{2} \mathrm{O}$ & 5.11 & 3.49 & 2.88 & 3.08 & 3.44 & 3.60 & 2.00 & 2.41 & 3.00 & 2.18 & 3.08 \\
\hline $\mathrm{P}_{2} \mathrm{O}_{5}$ & 0.11 & 0.20 & 0.13 & 0.16 & 0.20 & 0.14 & 0.20 & 0.17 & 0.13 & 0.19 & 0.22 \\
\hline $\mathrm{H}_{2} \mathrm{O}$ & 4.85 & 4.93 & 2.04 & 2.61 & 3.01 & 1.97 & 2.56 & 1.73 & 2.15 & 1.03 & 1.56 \\
\hline $\mathrm{CO}_{2}$ & 0.45 & 0.12 & 0.96 & 0.66 & 0.21 & 2.13 & 0.05 & 0.81 & 1.47 & 0.03 & 0.05 \\
\hline Summe & 99.74 & 99.72 & 99.85 & 99.86 & 99.75 & 99.80 & 99.73 & 99.87 & 99.82 & 99.75 & 99.75 \\
\hline $\mathrm{Li}$ & 54 & 52 & 34 & 24 & 37 & 12 & 48 & 21 & 37 & 69 & 43 \\
\hline $\mathrm{Be}$ & 3.6 & 2.7 & 3.1 & 2.3 & 2.7 & 2.4 & 1.9 & 1.8 & 2.5 & 3.1 & 2.2 \\
\hline $\mathrm{Sc}$ & 16.7 & 16.6 & 8.0 & 10.9 & 9.7 & 9.0 & 9.9 & 6.3 & 8.1 & 19.0 & 11.2 \\
\hline V & 128 & 143 & 55 & 78 & 90 & 64 & 70 & 61 & 60 & 146 & 101 \\
\hline $\mathrm{Cr}$ & 96 & 95 & 44 & 55 & 73 & 48 & 50 & 53 & 47 & 71 & 79 \\
\hline Co & 16 & 18 & 6 & 11 & 13 & 7 & 11 & 8 & 8 & 17 & 13 \\
\hline $\mathrm{Ni}$ & 41 & 38 & 14 & 22 & 29 & 17 & 23 & 17 & 18 & 36 & 28 \\
\hline $\mathrm{Cu}$ & 10 & 16 & 7 & 12 & 26 & 8 & 34 & 19 & 17 & 11 & 22 \\
\hline $\mathrm{Zn}$ & 128 & 155 & 65 & 81 & 94 & 49 & 94 & 56 & 80 & 108 & 92 \\
\hline$Y$ & 23 & 35 & 37 & 28 & 19 & 31 & 27 & 18 & 23 & 52 & 29 \\
\hline $\mathrm{Zr}$ & 168 & 129 & 216 & 152 & 189 & 188 & 180 & 157 & 189 & 341 & 212 \\
\hline $\mathrm{Nb}$ & 17 & 13 & 17 & 13 & 15 & 14 & 13 & 9 & 12 & 24 & 13 \\
\hline Mo & 0.2 & 0.5 & 0.2 & 0.2 & 0.1 & 0.2 & 0.1 & 0.3 & 0.2 & 0.2 & 0.0 \\
\hline $\mathrm{Cd}$ & 0.0 & 0.0 & 0.0 & 0.0 & 0.0 & 0.0 & 0.0 & 0.1 & 0.1 & 0.1 & 0.0 \\
\hline Sn & 0.1 & 0.1 & 2.5 & 2.9 & 0.1 & 1.5 & 1.2 & 0.0 & 0.1 & 4.1 & 0.1 \\
\hline $\mathrm{Sb}$ & 1.3 & 2.8 & 1.5 & 2.1 & 0.9 & 1.8 & 5.0 & 1.1 & 1.0 & 0.2 & 0.0 \\
\hline $\mathrm{Rb}$ & 155 & 138 & 140 & 145 & 91 & 163 & 101 & 54 & 99 & 170 & 112 \\
\hline $\mathrm{Sr}$ & 32 & 40 & 55 & 35 & 40 & 90 & 68 & 133 & 52 & 273 & 109 \\
\hline Cs & 11.8 & 8.6 & 7.1 & 8.4 & 7.0 & 8.3 & 569.3 & 5.7 & 6.8 & 11.9 & 9.0 \\
\hline $\mathrm{Ba}$ & 543 & 426 & 256 & 306 & 248 & 246 & 205 & 345 & 191 & 637 & 544 \\
\hline La & 27 & 30 & 36 & 34 & 22 & 34 & 29 & 21 & 24 & 69 & 30 \\
\hline $\mathrm{Ce}$ & 59 & 66 & 75 & 64 & 47 & 71 & 50 & 42 & 45 & 143 & 64 \\
\hline $\mathrm{Pr}$ & 7.3 & 9.0 & 9.4 & 8.4 & 6.1 & 8.6 & 7.5 & 5.5 & 6.2 & 17.9 & 7.9 \\
\hline $\mathrm{Nd}$ & 27.5 & 36.1 & 34.0 & 30.6 & 23.5 & 32.4 & 28.2 & 21.4 & 23.8 & 66.7 & 30.7 \\
\hline $\mathrm{Sm}$ & 5.5 & 7.9 & 7.4 & 6.3 & 4.8 & 6.5 & 5.6 & 4.3 & 4.9 & 13.5 & 6.4 \\
\hline $\mathrm{Eu}$ & 1.0 & 1.5 & 0.9 & 1.2 & 0.9 & 1.1 & 1.0 & 0.9 & 0.9 & 2.4 & 1.2 \\
\hline $\mathrm{Gd}$ & 4.7 & 7.5 & 7.4 & 6.2 & 4.4 & 5.8 & 5.1 & 3.9 & 4.6 & 12.7 & 6.2 \\
\hline $\mathrm{Tb}$ & 0.7 & 1.1 & 1.2 & 0.9 & 0.7 & 0.8 & 0.8 & 0.6 & 0.7 & 1.8 & 0.9 \\
\hline Dy & 4.6 & 6.5 & 7.1 & 5.6 & 4.0 & 4.9 & 4.5 & 3.5 & 4.4 & 10.3 & 5.5 \\
\hline Ho & 1.0 & 1.3 & 1.5 & 1.1 & 0.8 & 1.0 & 0.9 & 0.7 & 0.9 & 2.1 & 1.1 \\
\hline Er & 3.0 & 3.8 & 4.3 & 3.2 & 2.4 & 2.8 & 2.6 & 2.1 & 2.7 & 5.8 & 3.2 \\
\hline $\mathrm{Tm}$ & 0.4 & 0.5 & 0.6 & 0.4 & 0.4 & 0.4 & 0.4 & 0.3 & 0.4 & 0.8 & 0.4 \\
\hline $\mathrm{Yb}$ & 3.1 & 3.5 & 4.2 & 3.0 & 2.5 & 2.7 & 2.6 & 2.1 & 2.6 & 5.3 & 3.0 \\
\hline Lu & 0.5 & 0.5 & 0.6 & 0.4 & 0.4 & 0.4 & 0.4 & 0.3 & 0.4 & 0.8 & 0.4 \\
\hline $\mathrm{Hf}$ & 4.7 & 3.7 & 7.1 & 4.5 & 5.1 & 5.1 & 4.9 & 4.2 & 5.3 & 9.9 & 5.5 \\
\hline $\mathrm{Ta}$ & 1.1 & 0.9 & 1.3 & 1.1 & 0.6 & 1.0 & 0.9 & 0.3 & 0.6 & 1.8 & 0.7 \\
\hline W & 2.5 & 1.8 & 1.3 & 1.7 & 0.6 & 1.5 & 1.8 & 0.5 & 0.6 & 0.8 & 1.0 \\
\hline $\mathrm{Ti}$ & 1.1 & 0.7 & 0.7 & 0.7 & 0.7 & 0.7 & 0.4 & 0.4 & 0.6 & 1.0 & 0.7 \\
\hline $\mathrm{Pb}$ & 7.6 & 18.6 & 9.8 & 18.2 & 17.8 & 11.5 & 13.8 & 16.0 & 27.4 & 19.9 & 16.1 \\
\hline $\mathrm{Bi}$ & 0.3 & 0.3 & 0.3 & 0.3 & 0.4 & 0.2 & 0.6 & 0.2 & 0.4 & 0.2 & 0.5 \\
\hline Th & 12.5 & 12.2 & 15.5 & 12.7 & 8.0 & 12.5 & 10.4 & 7.7 & 10.3 & 21.8 & 10.6 \\
\hline$U$ & 2.1 & 3.1 & 3.1 & 2.7 & 1.9 & 1.9 & 1.9 & 1.5 & 2.9 & 4.9 & 2.0 \\
\hline Chondrit normi & Nerte (Ta) & Ind McLer & 1985) & & & & & & & & \\
\hline La & 73.1 & 81.4 & 99.1 & 92.0 & 59.6 & 93.0 & 79.6 & 58.2 & 64.3 & 187.9 & 82.7 \\
\hline $\mathrm{Ce}$ & 62.1 & 69.0 & 78.2 & 67.4 & 48.7 & 74.1 & 51.8 & 43.9 & 47.0 & 149.7 & 66.8 \\
\hline $\mathrm{Pr}$ & 53.5 & 65.5 & 68.8 & 61.5 & 44.5 & 62.9 & 54.8 & 40.4 & 45.5 & 131.0 & 57.9 \\
\hline $\mathrm{Nd}$ & 38.6 & 50.7 & 47.8 & 43.0 & 33.1 & 45.6 & 39.6 & 30.1 & 33.5 & 93.8 & 43.1 \\
\hline $\mathrm{Sm}$ & 23.6 & 34.4 & 32.1 & 27.2 & 20.8 & 28.0 & 24.3 & 18.6 & 21.1 & 58.4 & 27.7 \\
\hline $\mathrm{Eu}$ & 11.4 & 17.4 & 10.5 & 13.5 & 10.1 & 12.6 & 11.1 & 10.3 & 10.5 & 27.5 & 14.0 \\
\hline Gd & 15.5 & 24.5 & 24.1 & 20.1 & 14.3 & 18.8 & 16.5 & 12.7 & 15.0 & 41.4 & 20.2 \\
\hline $\mathrm{Tb}$ & 12.8 & 19.0 & 20.0 & 16.2 & 11.7 & 14.6 & 13.1 & 10.1 & 12.4 & 31.4 & 15.9 \\
\hline Dy & 12.2 & 17.0 & 18.7 & 14.7 & 10.6 & 12.7 & 11.8 & 9.2 & 11.5 & 27.0 & 14.4 \\
\hline Ho & 11.7 & 15.3 & 17.1 & 13.4 & 9.8 & 11.5 & 10.8 & 8.5 & 10.5 & 24.3 & 12.9 \\
\hline $\mathrm{Er}$ & 12.0 & 15.1 & 17.2 & 12.9 & 9.8 & 11.3 & 10.5 & 8.5 & 10.7 & 23.4 & 12.7 \\
\hline $\mathrm{Tm}$ & 12.4 & 14.8 & 17.2 & 12.6 & 10.0 & 11.3 & 10.7 & 8.5 & 10.7 & 22.0 & 12.4 \\
\hline $\mathrm{Yb}$ & 12.4 & 14.3 & 16.8 & 12.3 & 10.0 & 10.9 & 10.4 & 8.5 & 10.6 & 21.3 & 12.3 \\
\hline Lu & 11.9 & 13.6 & 16.0 & 11.4 & 9.6 & 10.5 & 10.1 & 8.3 & 10.1 & 20.1 & 11.5 \\
\hline $\mathrm{Cr} / \mathrm{V}$ & 0.75 & 0.66 & 0.80 & 0.71 & 0.81 & 0.75 & 0.71 & 0.86 & 0.78 & 0.49 & 0.78 \\
\hline $\mathrm{Th} / \mathrm{Sc}$ & 0.7 & 0.7 & 1.9 & 1.2 & 0.8 & 1.4 & 1.1 & 1.2 & 1.3 & 1.1 & 0.9 \\
\hline $\mathrm{La} / \mathrm{Th}$ & 2.1 & 2.5 & 2.3 & 2.7 & 2.7 & 2.7 & 2.8 & 2.8 & 2.3 & 3.2 & 2.9 \\
\hline $\mathrm{Ti} / \mathrm{Zr}$ & 49.0 & 62.2 & 20.6 & 38.7 & 40.7 & 27.0 & 30.0 & 35.4 & 25.3 & 25.0 & 38.7 \\
\hline $\mathrm{La} / \mathrm{Sc}$ & 1.6 & 1.8 & 4.5 & 3.1 & 2.3 & 3.8 & 2.9 & 3.4 & 2.9 & 3.6 & 2.7 \\
\hline $\mathrm{Cr} / \mathrm{Ni}$ & 2.3 & 2.5 & 3.1 & 2.5 & 2.5 & 2.8 & 2.2 & 3.2 & 2.6 & 1.9 & 2.8 \\
\hline $\mathrm{Y} / \mathrm{Ni}$ & 0.6 & 0.9 & 2.6 & 1.2 & 0.7 & 1.8 & 1.2 & 1.1 & 1.3 & 1.4 & 1.0 \\
\hline $\mathrm{Zr} / \mathrm{Sc}$ & 10.1 & 7.8 & 26.9 & 13.9 & 19.6 & 20.9 & 18.2 & 24.8 & 23.4 & 17.9 & 18.9 \\
\hline $\mathrm{Nb} / \mathrm{Y}$ & 0.7 & 0.4 & 0.5 & 0.5 & 0.8 & 0.5 & 0.5 & 0.5 & 0.5 & 0.5 & 0.5 \\
\hline $\mathrm{Th} / \mathrm{U}$ & 6.0 & 3.9 & 4.9 & 4.7 & 4.2 & 6.7 & 5.6 & 5.3 & 3.5 & 4.4 & 5.2 \\
\hline $\mathrm{La}_{N} / \mathrm{Sm}_{N}$ & 3.1 & 2.4 & 3.1 & 3.4 & 2.9 & 3.3 & 3.3 & 3.1 & 3.1 & 3.2 & 3.0 \\
\hline $\mathrm{La}_{N} / \mathrm{Yb}_{N}$ & 5.9 & 5.7 & 5.9 & 7.5 & 6.0 & 8.5 & 7.6 & 6.8 & 6.1 & 8.8 & 6.7 \\
\hline $\mathrm{Eu} / \mathrm{Eu}^{*}$ & 0.60 & 0.60 & 0.38 & 0.58 & 0.58 & 0.55 & 0.55 & 0.67 & 0.59 & 0.56 & 0.59 \\
\hline $\mathrm{Ce} / \mathrm{Ce}^{\star}$ & 1.07 & 1.01 & 1.02 & 0.96 & 1.01 & 1.03 & 0.83 & 0.95 & 0.92 & 1.02 & 1.02 \\
\hline$\Sigma$ LREE & 127 & 149 & 162 & 144 & 103 & 153 & 120 & 95 & 104 & 310 & 139 \\
\hline ¿REE & 146 & 175 & 190 & 166 & 119 & 172 & 138 & 109 & 121 & 352 & 161 \\
\hline
\end{tabular}


Sierras de Chepes

\begin{tabular}{|c|c|c|c|c|c|}
\hline Probe & A31-06 & A33-06 & A35-06 & A37-06 & A41-06 \\
\hline $\mathrm{SiO}_{2}$ & 63.59 & 75.32 & 76.43 & 60.42 & 72.68 \\
\hline $\mathrm{TiO}_{2}$ & 0.90 & 0.64 & 0.65 & 0.87 & 0.61 \\
\hline $\mathrm{Al}_{2} \mathrm{O}_{3}$ & 16.41 & 11.76 & 11.04 & 19.49 & 12.81 \\
\hline $\mathrm{Fe}_{2} \mathrm{O}_{3}$ & 6.58 & 3.37 & 3.81 & 5.83 & 4.87 \\
\hline $\mathrm{MnO}$ & 0.37 & 0.10 & 0.07 & 0.07 & 0.07 \\
\hline $\mathrm{MgO}$ & 2.39 & 1.18 & 1.57 & 2.55 & 2.11 \\
\hline $\mathrm{CaO}$ & 1.19 & 1.17 & 0.66 & 0.86 & 0.35 \\
\hline $\mathrm{Na}_{2} \mathrm{O}$ & 1.82 & 2.11 & 1.39 & 2.74 & 1.64 \\
\hline $\mathrm{K}_{\mathrm{O}} \mathrm{O}$ & 4.32 & 2.85 & 2.69 & 3.60 & 2.23 \\
\hline $\mathrm{P}_{2} \mathrm{O}_{5}$ & 0.19 & 0.19 & 0.15 & 0.16 & 0.14 \\
\hline $\mathrm{H}_{2} \mathrm{O}$ & 1.82 & 1.06 & 1.29 & 3.02 & 2.25 \\
\hline $\mathrm{CO}_{2}$ & 0.11 & 0.04 & 0.04 & 0.13 & 0.09 \\
\hline Summe & 99.67 & 99.79 & 99.79 & 99.74 & 99.84 \\
\hline $\mathrm{Li}$ & 50 & 35 & 32 & 20 & 21 \\
\hline $\mathrm{Be}$ & 2.9 & 1.0 & 2.9 & 3.5 & 1.3 \\
\hline $\mathrm{Sc}$ & 18.2 & 10.0 & 10.8 & 10.0 & 9.9 \\
\hline V & 137 & 79 & 81 & 122 & 75 \\
\hline $\mathrm{Cr}$ & 86 & 43 & 56 & 92 & 53 \\
\hline Co & 20 & 8 & 11 & 12 & 9 \\
\hline $\mathrm{Ni}$ & 38 & 15 & 26 & 35 & 24 \\
\hline $\mathrm{Cu}$ & 6 & 6 & 18 & 9 & 6 \\
\hline $\mathrm{Zn}$ & 118 & 65 & 75 & 62 & 64 \\
\hline$Y$ & 33 & 39 & 31 & 18 & 24 \\
\hline $\mathrm{Zr}$ & 192 & 279 & 173 & 172 & 116 \\
\hline $\mathrm{Nb}$ & 19 & 19 & 13 & 19 & 13 \\
\hline Mo & 0.3 & 0.2 & 0.3 & 0.3 & 0.3 \\
\hline $\mathrm{Cd}$ & 0.0 & 0.0 & 0.0 & 0.0 & 0.0 \\
\hline $\mathrm{Sn}$ & 4.3 & 3.9 & 2.7 & 0.1 & 1.3 \\
\hline $\mathrm{Sb}$ & 0.4 & 0.3 & 0.2 & 0.3 & 0.4 \\
\hline $\mathrm{Rb}$ & 213 & 139 & 125 & 50 & 107 \\
\hline $\mathrm{Sr}$ & 161 & 183 & 111 & 80 & 61 \\
\hline Cs & 14.7 & 6.5 & 6.1 & 3.9 & 3.3 \\
\hline $\mathrm{Ba}$ & 685 & 783 & 625 & 441 & 340 \\
\hline La & 48 & 52 & 40 & 18 & 27 \\
\hline $\mathrm{Ce}$ & 107 & 107 & 83 & 35 & 55 \\
\hline $\mathrm{Pr}$ & 12.0 & 13.1 & 10.1 & 4.9 & 6.8 \\
\hline $\mathrm{Nd}$ & 44.3 & 49.4 & 37.9 & 19.3 & 25.3 \\
\hline $\mathrm{Sm}$ & 8.6 & 9.7 & 7.5 & 4.0 & 5.0 \\
\hline Eu & 1.5 & 1.6 & 1.4 & 0.8 & 1.0 \\
\hline $\mathrm{Gd}$ & 7.7 & 9.1 & 7.2 & 3.7 & 4.4 \\
\hline $\mathrm{Tb}$ & 1.1 & 1.3 & 1.1 & 0.6 & 0.7 \\
\hline Dy & 6.6 & 7.8 & 6.3 & 3.8 & 3.9 \\
\hline $\mathrm{Ho}$ & 1.3 & 1.5 & 1.2 & 0.8 & 0.8 \\
\hline $\mathrm{Er}$ & 3.8 & 4.4 & 3.5 & 2.5 & 2.3 \\
\hline $\mathrm{Tm}$ & 0.6 & 0.6 & 0.5 & 0.4 & 0.3 \\
\hline $\mathrm{Yb}$ & 3.7 & 4.1 & 3.2 & 2.6 & 2.1 \\
\hline Lu & 0.5 & 0.6 & 0.5 & 0.4 & 0.3 \\
\hline $\mathrm{Hf}$ & 5.6 & 8.3 & 5.3 & 4.8 & 3.1 \\
\hline $\mathrm{Ta}$ & 1.5 & 1.5 & 0.9 & 1.3 & 0.9 \\
\hline W & 3.2 & 2.3 & 2.4 & 3.3 & 1.7 \\
\hline $\mathrm{Ti}$ & 1.1 & 0.7 & 0.7 & 0.5 & 0.4 \\
\hline $\mathrm{Pb}$ & 19.6 & 30.3 & 16.0 & 8.5 & 15.2 \\
\hline $\mathrm{Bi}$ & 0.4 & 0.2 & 0.2 & 0.1 & 0.2 \\
\hline Th & 17.1 & 16.8 & 11.7 & 5.8 & 8.2 \\
\hline U & 3.2 & 3.9 & 2.4 & 1.2 & 1.9 \\
\hline
\end{tabular}

Chondrit normierte Werte (Taylor und McLennan 1985)

$\begin{array}{crrrrr}\mathrm{La} & 129.5 & 141.5 & 109.4 & 48.6 & 74.1 \\ \mathrm{Ce} & 111.7 & 111.8 & 87.0 & 37.1 & 57.9 \\ \mathrm{Pr} & 87.9 & 95.9 & 73.9 & 35.9 & 49.5 \\ \mathrm{Nd} & 62.4 & 69.5 & 53.3 & 27.1 & 35.6 \\ \mathrm{Sm} & 37.1 & 41.8 & 32.4 & 17.4 & 21.7 \\ \mathrm{Eu} & 16.9 & 18.8 & 16.1 & 9.2 & 11.2 \\ \mathrm{Gd} & 25.2 & 29.6 & 23.7 & 12.1 & 14.4 \\ \mathrm{~Tb} & 19.2 & 22.6 & 18.3 & 10.3 & 11.6 \\ \mathrm{Dy} & 17.3 & 20.4 & 16.4 & 10.0 & 10.3 \\ \mathrm{Ho} & 15.5 & 18.2 & 14.5 & 9.6 & 9.3 \\ \mathrm{Er} & 15.4 & 17.7 & 14.0 & 10.1 & 9.1 \\ \mathrm{Tm} & 15.5 & 17.1 & 13.6 & 10.4 & 9.1 \\ \mathrm{Yb} & 15.0 & 16.6 & 12.8 & 10.4 & 8.5 \\ \mathrm{Lu} & 14.4 & 16.2 & 12.0 & 10.2 & 8.0 \\ & & & & & \\ \mathrm{Cr} / \mathrm{N} & 0.63 & 0.54 & 0.69 & 0.76 & 0.71 \\ \mathrm{Th} / \mathrm{Sc} & 0.94 & 1.69 & 1.09 & 0.58 & 0.83 \\ \mathrm{La} / \mathrm{Th} & 2.8 & 3.1 & 3.4 & 3.0 & 3.3 \\ \mathrm{Ti} / \mathrm{Zr} & 46.7 & 23.0 & 37.3 & 50.5 & 52.1 \\ \mathrm{La} / \mathrm{Sc} & 2.6 & 5.2 & 3.7 & 1.8 & 2.8 \\ \mathrm{Cr} / \mathrm{Ni} & 2.2 & 2.9 & 2.2 & 2.6 & 2.2 \\ \mathrm{Y} / \mathrm{Ni} & 0.9 & 2.6 & 1.2 & 0.5 & 1.0 \\ \mathrm{Zr} / \mathrm{Sc} & 10.5 & 28.0 & 16.1 & 17.1 & 11.8 \\ \mathrm{Nb} / \mathrm{Y} & 0.6 & 0.5 & 0.4 & 1.0 & 0.6 \\ \mathrm{Th} / \mathrm{U} & 5.4 & 4.3 & 4.9 & 5.0 & 4.3 \\ \mathrm{La} / \mathrm{Sm} & 3.5 & 3.4 & 3.4 & 2.8 & 3.4 \\ \mathrm{La} / \mathrm{Sb} \mathrm{w}_{N} & 8.6 & 8.5 & 8.6 & 4.7 & 8.7 \\ \mathrm{Eu} / \mathrm{Eu} & 0.55 & 0.53 & 0.58 & 0.63 & 0.63 \\ \mathrm{Ce} / \mathrm{Ce} & 1.10 & 1.00 & 1.01 & 0.93 & 1.00 \\ \Sigma \mathrm{LREE} & 219 & 231 & 179 & 82 & 120 \\ \Sigma \mathrm{REE} & 246 & 262 & 204 & 97 & 135 \\ & & & & & \end{array}$


Sierras de Córdoba

\begin{tabular}{|c|c|c|c|c|c|c|c|c|}
\hline Probe & A60-06 & A61-06 & A62-06 & A63-06 & A64-06 & A65-06 & A66-06 & A68-06 \\
\hline $\mathrm{SiO}_{2}$ & 67.24 & 69.34 & 74.89 & 72.01 & 68.04 & 59.93 & 74.50 & 69.89 \\
\hline $\mathrm{TiO}_{2}$ & 1.16 & 0.85 & 0.65 & 0.55 & 0.80 & 0.87 & 0.78 & 1.00 \\
\hline $\mathrm{Al}_{2} \mathrm{O}_{3}$ & 13.76 & 13.48 & 11.40 & 11.74 & 13.88 & 17.53 & 11.10 & 13.38 \\
\hline $\mathrm{Fe}_{2} \mathrm{O}_{3}$ & 6.23 & 5.29 & 3.86 & 5.23 & 5.46 & 7.72 & 4.32 & 5.04 \\
\hline $\mathrm{MnO}$ & 0.11 & 0.08 & 0.06 & 0.13 & 0.08 & 0.16 & 0.05 & 0.03 \\
\hline $\mathrm{MgO}$ & 3.11 & 2.66 & 1.58 & 2.94 & 2.56 & 3.07 & 1.89 & 2.02 \\
\hline $\mathrm{CaO}$ & 2.01 & 2.04 & 1.64 & 1.58 & 2.03 & 3.53 & 1.94 & 0.59 \\
\hline $\mathrm{Na}_{2} \mathrm{O}$ & 1.97 & 2.03 & 2.45 & 1.53 & 2.38 & 2.93 & 2.09 & 2.58 \\
\hline $\mathrm{K}_{2} \mathrm{O}$ & 2.60 & 2.91 & 2.03 & 2.47 & 2.98 & 2.49 & 2.04 & 2.46 \\
\hline $\mathrm{P}_{2} \mathrm{O}_{5}$ & 0.08 & 0.22 & 0.16 & 0.11 & 0.18 & 0.17 & 0.15 & 0.26 \\
\hline $\mathrm{H}_{2} \mathrm{O}$ & 1.35 & 0.81 & 1.05 & 1.48 & 1.29 & 1.21 & 0.92 & 2.23 \\
\hline $\mathrm{CO}_{2}$ & 0.05 & 0.03 & 0.04 & 0.04 & 0.05 & 0.11 & 0.05 & 0.21 \\
\hline Summe & 99.68 & 99.74 & 99.81 & 99.81 & 99.73 & 99.73 & 99.83 & 99.69 \\
\hline $\mathrm{Li}$ & 22 & 11 & 65 & 89 & 44 & 23 & 19 & 40 \\
\hline $\mathrm{Be}$ & 0.3 & 1.0 & 1.9 & 1.8 & 1.3 & 0.7 & 0.5 & 2.4 \\
\hline $\mathrm{Sc}$ & 16.2 & 13.5 & 10.1 & 12.7 & 14.2 & 18.4 & 11.8 & 14.4 \\
\hline V & 130 & 122 & 84 & 98 & 120 & 140 & 99 & 103 \\
\hline $\mathrm{Cr}$ & 97 & 80 & 59 & 74 & 80 & 104 & 64 & 73 \\
\hline Co & 17 & 13 & 9 & 15 & 13 & 16 & 10 & 12 \\
\hline $\mathrm{Ni}$ & 38 & 33 & 19 & 32 & 29 & 36 & 23 & 22 \\
\hline $\mathrm{Cu}$ & 9 & 23 & 12 & 7 & 10 & 16 & 12 & 19 \\
\hline $\mathrm{Zn}$ & 120 & 82 & 58 & 95 & 88 & 111 & 73 & 81 \\
\hline$Y$ & 37 & 35 & 26 & 22 & 29 & 36 & 27 & 48 \\
\hline $\mathrm{Zr}$ & 121 & 109 & 152 & 111 & 141 & 121 & 189 & 314 \\
\hline $\mathrm{Nb}$ & 6 & 13 & 11 & 11 & 14 & 19 & 13 & 17 \\
\hline Mo & 0.2 & 0.2 & 0.2 & 0.1 & 0.1 & 0.2 & 0.2 & 0.2 \\
\hline $\mathrm{Cd}$ & 0.1 & 0.1 & 0.1 & 0.1 & 0.1 & 0.1 & 0.0 & 0.0 \\
\hline Sn & 0.7 & 0.7 & 2.8 & 2.8 & 1.6 & 0.9 & 1.1 & 4.0 \\
\hline $\mathrm{Sb}$ & 0.6 & 0.1 & 0.2 & 0.1 & 0.1 & 0.5 & 0.2 & 1.9 \\
\hline $\mathrm{Rb}$ & 113 & 121 & 93 & 137 & 146 & 138 & 105 & 115 \\
\hline $\mathrm{Sr}$ & 184 & 185 & 160 & 128 & 181 & 247 & 164 & 90 \\
\hline Cs & 1.8 & 2.7 & 22.0 & 15.9 & 6.1 & 4.6 & 3.9 & 6.3 \\
\hline $\mathrm{Ba}$ & 487 & 490 & 445 & 282 & 498 & 176 & 272 & 372 \\
\hline La & 49 & 30 & 31 & 24 & 36 & 40 & 41 & 59 \\
\hline $\mathrm{Ce}$ & 102 & 64 & 69 & 46 & 73 & 82 & 86 & 127 \\
\hline $\operatorname{Pr}$ & 12.4 & 8.3 & 8.1 & 6.0 & 9.3 & 10.0 & 10.5 & 16.1 \\
\hline $\mathrm{Nd}$ & 47.3 & 32.6 & 30.4 & 22.3 & 35.2 & 37.0 & 39.8 & 61.0 \\
\hline $\mathrm{Sm}$ & 8.7 & 7.2 & 6.1 & 4.4 & 7.0 & 7.2 & 8.0 & 12.5 \\
\hline $\mathrm{Eu}$ & 1.7 & 1.4 & 1.2 & 0.8 & 1.3 & 1.3 & 1.3 & 2.0 \\
\hline $\mathrm{Gd}$ & 7.6 & 7.0 & 5.6 & 3.8 & 6.1 & 6.2 & 7.1 & 11.5 \\
\hline $\mathrm{Tb}$ & 1.1 & 1.1 & 0.9 & 0.6 & 0.9 & 1.0 & 1.0 & 1.7 \\
\hline Dy & 7.0 & 5.9 & 5.2 & 3.4 & 5.0 & 5.7 & 5.9 & 9.8 \\
\hline Ho & 1.5 & 1.2 & 1.1 & 0.7 & 1.0 & 1.1 & 1.1 & 1.9 \\
\hline Er & 4.6 & 3.4 & 3.1 & 2.0 & 2.6 & 3.2 & 3.0 & 5.4 \\
\hline $\mathrm{Tm}$ & 0.7 & 0.5 & 0.4 & 0.3 & 0.4 & 0.5 & 0.4 & 0.8 \\
\hline $\mathrm{Yb}$ & 4.4 & 3.3 & 2.9 & 1.9 & 2.3 & 3.1 & 2.8 & 5.1 \\
\hline Lu & 0.7 & 0.5 & 0.4 & 0.3 & 0.3 & 0.5 & 0.4 & 0.7 \\
\hline $\mathrm{Hf}$ & 3.5 & 2.7 & 4.6 & 2.8 & 3.7 & 3.2 & 5.5 & 11.0 \\
\hline $\mathrm{Ta}$ & 0.4 & 0.5 & 1.0 & 0.8 & 1.0 & 1.1 & 0.9 & 1.3 \\
\hline W & 0.3 & 0.5 & 1.3 & 0.5 & 0.8 & 0.5 & 0.3 & 2.0 \\
\hline $\mathrm{Ti}$ & 0.6 & 0.5 & 0.5 & 0.6 & 0.8 & 0.6 & 0.6 & 0.6 \\
\hline $\mathrm{Pb}$ & 17.0 & 16.2 & 15.9 & 20.4 & 18.5 & 13.5 & 14.1 & 9.8 \\
\hline $\mathrm{Bi}$ & 0.0 & 0.0 & 0.1 & 0.3 & 0.1 & 0.1 & 0.1 & 0.1 \\
\hline Th & 16.7 & 8.4 & 9.8 & 8.3 & 10.9 & 14.0 & 12.5 & 19.6 \\
\hline U & 1.3 & 1.4 & 1.9 & 2.0 & 2.3 & 1.8 & 2.7 & 3.6 \\
\hline \multicolumn{9}{|c|}{ Chondrit normierte Werte (Taylor und McLennan 1985) } \\
\hline La & 132.6 & 81.7 & 85.6 & 65.6 & 97.4 & 109.8 & 112.2 & 161.3 \\
\hline $\mathrm{Ce}$ & 106.5 & 66.6 & 71.7 & 48.5 & 76.3 & 85.5 & 90.1 & 132.6 \\
\hline $\mathrm{Pr}$ & 90.7 & 60.2 & 58.9 & 43.7 & 67.8 & 72.8 & 77.0 & 117.3 \\
\hline $\mathrm{Nd}$ & 66.5 & 45.9 & 42.7 & 31.4 & 49.5 & 52.0 & 56.0 & 85.8 \\
\hline $\mathrm{Sm}$ & 37.8 & 31.1 & 26.3 & 18.9 & 30.1 & 31.0 & 34.4 & 54.0 \\
\hline $\mathrm{Eu}$ & 19.9 & 16.2 & 13.5 & 9.0 & 15.4 & 14.8 & 14.9 & 23.2 \\
\hline Gd & 24.7 & 22.8 & 18.2 & 12.4 & 20.0 & 20.2 & 23.1 & 37.4 \\
\hline $\mathrm{Tb}$ & 18.8 & 18.3 & 14.9 & 10.0 & 15.5 & 16.9 & 17.6 & 28.7 \\
\hline Dy & 18.4 & 15.4 & 13.7 & 8.8 & 13.0 & 15.1 & 15.4 & 25.8 \\
\hline Ho & 17.5 & 13.8 & 12.6 & 8.1 & 11.3 & 13.3 & 13.1 & 22.6 \\
\hline Er & 18.3 & 13.5 & 12.3 & 8.0 & 10.4 & 12.8 & 12.2 & 21.9 \\
\hline $\mathrm{Tm}$ & 18.7 & 13.6 & 12.3 & 7.9 & 10.2 & 12.8 & 11.9 & 21.5 \\
\hline $\mathrm{Yb}$ & 17.9 & 13.2 & 11.7 & 7.6 & 9.4 & 12.5 & 11.4 & 20.5 \\
\hline Lu & 17.5 & 12.7 & 11.1 & 7.4 & 9.0 & 11.9 & 11.3 & 19.3 \\
\hline $\mathrm{Cr} / \mathrm{N}$ & 0.74 & 0.66 & 0.71 & 0.75 & 0.67 & 0.74 & 0.64 & 0.71 \\
\hline $\mathrm{Th} / \mathrm{Sc}$ & 1.03 & 0.62 & 0.97 & 0.65 & 0.77 & 0.76 & 1.06 & 1.36 \\
\hline $\mathrm{La} / \mathrm{Th}$ & 2.9 & 3.6 & 3.2 & 2.9 & 3.3 & 2.9 & 3.3 & 3.0 \\
\hline $\mathrm{Ti} / \mathrm{Zr}$ & 96.2 & 77.4 & 42.8 & 49.6 & 56.2 & 71.9 & 41.1 & 31.7 \\
\hline $\mathrm{La} / \mathrm{Sc}$ & 3.0 & 2.2 & 3.1 & 1.9 & 2.5 & 2.2 & 3.5 & 4.1 \\
\hline $\mathrm{Cr} / \mathrm{Ni}$ & 2.6 & 2.4 & 3.1 & 2.3 & 2.7 & 2.9 & 2.8 & 3.1 \\
\hline $\mathrm{Y} / \mathrm{Ni}$ & 0.98 & 1.06 & 1.39 & 0.70 & 0.98 & 1.00 & 1.21 & 2.15 \\
\hline $\mathrm{Zr} / \mathrm{Sc}$ & 7.5 & 8.1 & 15.1 & 8.7 & 9.9 & 6.6 & 16.0 & 21.9 \\
\hline $\mathrm{Nb} / \mathrm{Y}$ & 0.2 & 0.4 & 0.4 & 0.5 & 0.5 & 0.5 & 0.5 & 0.3 \\
\hline $\mathrm{Th} / \mathrm{U}$ & 12.5 & 6.0 & 5.0 & 4.2 & 4.8 & 7.6 & 4.6 & 5.5 \\
\hline $\mathrm{La}_{N} / \mathrm{Sm}_{N}$ & 3.5 & 2.6 & 3.3 & 3.5 & 3.2 & 3.5 & 3.3 & 3.0 \\
\hline $\mathrm{La}_{N} / \mathrm{Yb}_{N}$ & 7.4 & 6.2 & 7.3 & 8.7 & 10.4 & 8.8 & 9.8 & 7.9 \\
\hline $\mathrm{Eu} / \mathrm{Eu}^{*}$ & 0.65 & 0.61 & 0.62 & 0.59 & 0.63 & 0.59 & 0.53 & 0.52 \\
\hline $\mathrm{Ce} / \mathrm{Ce}^{*}$ & 1.01 & 0.99 & 1.06 & 0.94 & 0.98 & 1.00 & 1.01 & 1.01 \\
\hline$\Sigma$ LREE & 219 & 142 & 144 & 103 & 160 & 176 & 186 & 276 \\
\hline ¿REE & 248 & 166 & 165 & 117 & 180 & 199 & 209 & 315 \\
\hline
\end{tabular}


Sierras de Córdoba

\begin{tabular}{|c|c|c|c|c|c|c|c|c|}
\hline Probe & A70-06 & A74-06 & A75-06 & A76-06 & A77-06 & A78-06 & A79-06 & A80-06 \\
\hline $\mathrm{SiO}_{2}$ & 70.27 & 69.47 & 70.87 & 60.35 & 69.12 & 67.38 & 71.39 & 63.65 \\
\hline $\mathrm{TiO}_{2}$ & 1.09 & 0.64 & 0.67 & 1.04 & 0.75 & 0.87 & 0.52 & 1.16 \\
\hline $\mathrm{Al}_{2} \mathrm{O}_{3}$ & 12.55 & 13.79 & 12.74 & 17.14 & 13.88 & 14.85 & 13.68 & 14.83 \\
\hline $\mathrm{Fe}_{2} \mathrm{O}_{3}$ & 5.24 & 4.66 & 4.48 & 8.54 & 5.71 & 6.17 & 3.52 & 7.14 \\
\hline $\mathrm{MnO}$ & 0.09 & 0.07 & 0.09 & 0.15 & 0.10 & 0.07 & 0.06 & 0.13 \\
\hline $\mathrm{MgO}$ & 1.86 & 2.06 & 2.28 & 4.19 & 2.46 & 2.56 & 1.33 & 2.55 \\
\hline $\mathrm{CaO}$ & 1.80 & 1.68 & 3.17 & 1.47 & 2.14 & 1.17 & 1.30 & 2.45 \\
\hline $\mathrm{Na}_{2} \mathrm{O}$ & 3.26 & 2.53 & 1.93 & 1.81 & 2.55 & 1.47 & 2.40 & 2.34 \\
\hline $\mathrm{K}_{2} \mathrm{O}$ & 2.09 & 3.31 & 2.20 & 3.18 & 2.00 & 3.53 & 3.98 & 3.68 \\
\hline $\mathrm{P}_{2} \mathrm{O}_{5}$ & 0.30 & 0.30 & 0.18 & 0.11 & 0.18 & 0.12 & 0.14 & 0.45 \\
\hline $\mathrm{H}_{2} \mathrm{O}$ & 1.11 & 1.23 & 1.07 & 1.62 & 0.82 & 1.47 & 1.03 & 1.23 \\
\hline $\mathrm{CO}_{2}$ & 0.04 & 0.06 & 0.06 & 0.08 & 0.07 & 0.06 & 0.15 & 0.24 \\
\hline Summe & 99.69 & 99.79 & 99.75 & 99.68 & 99.78 & 99.73 & 99.50 & 99.84 \\
\hline $\mathrm{Li}$ & 29 & 71 & 40 & 24 & 16 & 17 & 36 & 34 \\
\hline $\mathrm{Be}$ & 1.8 & 2.9 & 2.2 & 0.6 & 1.0 & 0.8 & 0.6 & 3.2 \\
\hline $\mathrm{Sc}$ & 13.5 & 13.4 & 10.9 & 22.5 & 16.1 & 15.1 & 18.8 & 7.3 \\
\hline V & 96 & 95 & 80 & 158 & 117 & 117 & 134 & 66 \\
\hline $\mathrm{Cr}$ & 69 & 65 & 61 & 116 & 76 & 80 & 43 & 60 \\
\hline Co & 10 & 12 & 11 & 20 & 15 & 14 & 15 & 8 \\
\hline $\mathrm{Ni}$ & 18 & 24 & 21 & 44 & 30 & 31 & 21 & 16 \\
\hline $\mathrm{Cu}$ & 28 & 11 & 11 & 44 & 27 & 13 & 33 & 34 \\
\hline $\mathrm{Zn}$ & 68 & 84 & 71 & 129 & 99 & 103 & 109 & 66 \\
\hline$Y$ & 54 & 24 & 25 & 46 & 42 & 26 & 45 & 17 \\
\hline $\mathrm{Zr}$ & 469 & 134 & 162 & 88 & 131 & 133 & 101 & 106 \\
\hline $\mathrm{Nb}$ & 16 & 19 & 10 & 16 & 13 & 16 & 24 & 15 \\
\hline Mo & 0.1 & 0.2 & 0.1 & 0.1 & 0.1 & 0.1 & 0.3 & 0.1 \\
\hline $\mathrm{Cd}$ & 0.1 & 0.1 & 0.0 & 0.1 & 0.1 & 0.0 & 0.1 & 0.0 \\
\hline Sn & 3.0 & 2.4 & 3.0 & 1.9 & 0.6 & 3.0 & 1.8 & 2.0 \\
\hline $\mathrm{Sb}$ & 0.1 & 0.2 & 0.1 & 0.1 & 0.1 & 0.0 & 0.0 & 0.1 \\
\hline $\mathrm{Rb}$ & 96 & 189 & 110 & 139 & 102 & 154 & 179 & 153 \\
\hline $\mathrm{Sr}$ & 185 & 139 & 187 & 164 & 274 & 100 & 216 & 95 \\
\hline Cs & 5.9 & 15.3 & 5.5 & 2.7 & 2.2 & 3.6 & 5.0 & 2.8 \\
\hline $\mathrm{Ba}$ & 274 & 302 & 469 & 474 & 364 & 438 & 553 & 262 \\
\hline La & 56 & 39 & 31 & 41 & 35 & 44 & 50 & 37 \\
\hline $\mathrm{Ce}$ & 123 & 82 & 66 & 84 & 74 & 92 & 107 & 83 \\
\hline $\operatorname{Pr}$ & 15.5 & 10.3 & 8.4 & 10.4 & 9.4 & 11.1 & 13.0 & 10.4 \\
\hline $\mathrm{Nd}$ & 59.9 & 37.9 & 31.9 & 39.9 & 36.3 & 41.7 & 50.1 & 40.0 \\
\hline Sm & 12.0 & 7.7 & 6.7 & 7.8 & 7.4 & 8.1 & 10.1 & 8.2 \\
\hline Eu & 1.8 & 1.2 & 1.1 & 1.4 & 1.5 & 1.4 & 1.7 & 1.0 \\
\hline $\mathrm{Gd}$ & 10.5 & 6.9 & 6.3 & 6.7 & 6.7 & 6.7 & 9.5 & 6.2 \\
\hline $\mathrm{Tb}$ & 1.6 & 1.0 & 0.9 & 1.1 & 1.1 & 0.9 & 1.5 & 0.7 \\
\hline Dy & 9.1 & 5.5 & 5.2 & 6.8 & 6.6 & 5.0 & 8.3 & 3.4 \\
\hline $\mathrm{Ho}$ & 1.8 & 1.0 & 1.0 & 1.4 & 1.4 & 0.9 & 1.6 & 0.6 \\
\hline Er & 5.1 & 2.6 & 2.9 & 4.1 & 4.1 & 2.3 & 4.2 & 1.5 \\
\hline $\mathrm{Tm}$ & 0.7 & 0.3 & 0.4 & 0.6 & 0.6 & 0.3 & 0.6 & 0.2 \\
\hline $\mathrm{Yb}$ & 5.0 & 2.3 & 2.9 & 3.8 & 3.9 & 2.1 & 4.1 & 1.7 \\
\hline Lu & 0.7 & 0.3 & 0.4 & 0.6 & 0.6 & 0.3 & 0.6 & 0.3 \\
\hline $\mathrm{Hf}$ & 12.0 & 4.0 & 4.9 & 2.2 & 3.4 & 3.5 & 2.4 & 2.8 \\
\hline $\mathrm{Ta}$ & 1.1 & 1.5 & 0.9 & 0.8 & 0.7 & 0.8 & 1.3 & 0.7 \\
\hline W & 0.6 & 1.4 & 3.1 & 0.2 & 0.4 & 0.5 & 0.7 & 0.4 \\
\hline $\mathrm{Ti}$ & 0.5 & 1.0 & 0.6 & 0.6 & 0.5 & 0.7 & 0.9 & 0.7 \\
\hline $\mathrm{Pb}$ & 12.0 & 20.6 & 15.3 & 14.8 & 17.5 & 15.4 & 18.4 & 21.3 \\
\hline $\mathrm{Bi}$ & 0.5 & 0.5 & 0.2 & 0.0 & 0.0 & 0.0 & 0.1 & 0.1 \\
\hline Th & 17.9 & 12.3 & 9.4 & 13.7 & 10.5 & 14.3 & 14.5 & 19.8 \\
\hline$U$ & 4.1 & 4.8 & 1.1 & 1.7 & 2.1 & 1.9 & 2.2 & 2.6 \\
\hline \multicolumn{9}{|c|}{ Chondrit normierte Werte (Taylor und McLennan 1985) } \\
\hline La & 152.8 & 106.4 & 83.6 & 110.5 & 95.7 & 119.2 & 136.6 & 102.0 \\
\hline $\mathrm{Ce}$ & 128.5 & 85.3 & 68.6 & 87.8 & 77.5 & 96.5 & 111.6 & 86.5 \\
\hline $\mathrm{Pr}$ & 113.3 & 74.9 & 61.6 & 76.3 & 68.6 & 80.8 & 95.2 & 76.1 \\
\hline $\mathrm{Nd}$ & 84.2 & 53.3 & 44.9 & 56.1 & 51.0 & 58.7 & 70.5 & 56.3 \\
\hline $\mathrm{Sm}$ & 52.0 & 33.5 & 29.1 & 33.7 & 31.9 & 35.0 & 43.6 & 35.7 \\
\hline $\mathrm{Eu}$ & 20.8 & 13.7 & 13.1 & 16.0 & 17.8 & 16.2 & 19.8 & 11.4 \\
\hline Gd & 34.4 & 22.5 & 20.4 & 21.9 & 22.0 & 22.0 & 30.9 & 20.3 \\
\hline $\mathrm{Tb}$ & 27.2 & 16.9 & 15.8 & 18.2 & 18.3 & 16.2 & 25.4 & 12.8 \\
\hline Dy & 23.8 & 14.5 & 13.7 & 17.8 & 17.3 & 13.1 & 21.9 & 8.9 \\
\hline Ho & 21.5 & 11.7 & 12.1 & 16.9 & 16.4 & 10.6 & 18.5 & 6.9 \\
\hline $\mathrm{Er}$ & 20.6 & 10.4 & 11.8 & 16.5 & 16.4 & 9.3 & 16.8 & 6.1 \\
\hline $\mathrm{Tm}$ & 20.6 & 9.7 & 11.6 & 16.2 & 16.3 & 8.9 & 16.8 & 6.6 \\
\hline $\mathrm{Yb}$ & 20.1 & 9.4 & 11.5 & 15.3 & 15.8 & 8.4 & 16.6 & 7.0 \\
\hline Lu & 19.6 & 8.6 & 10.8 & 14.5 & 15.0 & 8.3 & 15.9 & 7.2 \\
\hline $\mathrm{Cr} N \mathrm{~N}$ & 0.72 & 0.68 & 0.76 & 0.74 & 0.65 & 0.68 & 0.32 & 0.91 \\
\hline $\mathrm{Th} / \mathrm{Sc}$ & 1.32 & 0.92 & 0.87 & 0.61 & 0.65 & 0.95 & 0.77 & 2.71 \\
\hline $\mathrm{La} / \mathrm{Th}$ & 3.1 & 3.2 & 3.3 & 3.0 & 3.3 & 3.1 & 3.4 & 1.9 \\
\hline $\mathrm{Ti} / \mathrm{Zr}$ & 23.2 & 47.7 & 41.4 & 117.9 & 57.0 & 65.2 & 51.8 & 109.4 \\
\hline $\mathrm{La} / \mathrm{Sc}$ & 4.1 & 2.9 & 2.8 & 1.8 & 2.2 & 2.9 & 2.7 & 5.1 \\
\hline $\mathrm{Cr} / \mathrm{Ni}$ & 2.6 & 2.5 & 5.5 & 1.7 & 2.6 & 1.4 & 2.8 & 4.7 \\
\hline $\mathrm{Y} / \mathrm{Ni}$ & 2.98 & 0.99 & 1.19 & 1.04 & 1.37 & 0.84 & 2.13 & 1.02 \\
\hline $\mathrm{Zr} / \mathrm{Sc}$ & 34.7 & 10.0 & 14.9 & 3.9 & 8.1 & 8.8 & 5.4 & 14.4 \\
\hline $\mathrm{Nb} / \mathrm{Y}$ & 0.3 & 0.8 & 0.4 & 0.4 & 0.3 & 0.6 & 0.5 & 0.9 \\
\hline $\mathrm{Th} / \mathrm{U}$ & 4.3 & 2.6 & 8.7 & 8.0 & 5.0 & 7.5 & 6.5 & 7.6 \\
\hline $\mathrm{La}_{N} / \mathrm{Sm}_{N}$ & 2.9 & 3.2 & 2.9 & 3.3 & 3.0 & 3.4 & 3.1 & 2.9 \\
\hline $\mathrm{La}_{N} / \mathrm{Yb}_{N}$ & 7.6 & 11.3 & 7.3 & 7.2 & 6.1 & 14.1 & 8.2 & 14.6 \\
\hline $\mathrm{Eu} / \mathrm{Eu}^{*}$ & 0.49 & 0.50 & 0.54 & 0.59 & 0.67 & 0.58 & 0.54 & 0.42 \\
\hline $\mathrm{Ce} / \mathrm{Ce}^{*}$ & 1.03 & 1.01 & 1.01 & 1.00 & 1.00 & 1.03 & 1.02 & 1.03 \\
\hline ¿LREE & 266 & 177 & 143 & 183 & 162 & 197 & 230 & 179 \\
\hline ¿REE & 303 & 198 & 165 & 209 & 189 & 217 & 262 & 195 \\
\hline
\end{tabular}


Sierra Norte

\begin{tabular}{|c|c|c|c|c|c|c|c|c|c|c|}
\hline Probe & A81-06 & A100-06 & A101-06 & A102-06 & A103-06 & A104-06 & A105-06 & A106-06 & A107-06 & A108-06 \\
\hline $\mathrm{SiO}_{2}$ & 74.30 & 74.20 & 70.86 & 46.74 & 60.65 & 75.00 & 71.04 & 60.62 & 70.75 & 68.56 \\
\hline $\mathrm{TiO}_{2}$ & 0.79 & 0.73 & 0.78 & 0.55 & 0.88 & 0.48 & 0.62 & 0.80 & 0.58 & 0.72 \\
\hline $\mathrm{Al}_{2} \mathrm{O}_{3}$ & 11.50 & 11.61 & 12.90 & 15.07 & 17.54 & 12.16 & 13.46 & 16.99 & 13.93 & 15.01 \\
\hline $\mathrm{Fe}_{2} \mathrm{O}_{3}$ & 4.65 & 4.28 & 4.90 & 9.87 & 7.60 & 2.95 & 4.32 & 6.85 & 4.51 & 4.96 \\
\hline $\mathrm{MnO}$ & 0.08 & 0.07 & 0.07 & 0.13 & 0.13 & 0.07 & 0.07 & 0.10 & 0.07 & 0.07 \\
\hline $\mathrm{MgO}$ & 1.71 & 1.41 & 1.98 & 10.63 & 3.59 & 1.14 & 1.91 & 2.85 & 1.59 & 2.03 \\
\hline $\mathrm{CaO}$ & 0.94 & 1.14 & 1.59 & 13.95 & 0.84 & 2.38 & 1.21 & 1.48 & 1.02 & 0.36 \\
\hline $\mathrm{Na}_{2} \mathrm{O}$ & 1.32 & 1.65 & 2.60 & 1.09 & 1.52 & 2.91 & 2.54 & 2.06 & 2.21 & 2.28 \\
\hline $\mathrm{K}_{2} \mathrm{O}$ & 2.53 & 3.01 & 2.42 & 0.14 & 4.95 & 1.65 & 2.95 & 4.68 & 2.75 & 2.71 \\
\hline $\mathrm{P}_{2} \mathrm{O}_{5}$ & 0.12 & 0.19 & 0.19 & 0.03 & 0.15 & 0.16 & 0.17 & 0.17 & 0.14 & 0.18 \\
\hline $\mathrm{H}_{2} \mathrm{O}$ & 1.75 & 1.46 & 1.42 & 1.58 & 1.77 & 0.83 & 1.40 & 2.42 & 2.08 & 2.83 \\
\hline $\mathrm{CO}_{2}$ & 0.10 & 0.03 & 0.06 & 0.05 & 0.07 & 0.04 & 0.07 & 0.74 & 0.15 & 0.11 \\
\hline Summe & 99.79 & 99.76 & 99.77 & 99.83 & 99.69 & 99.77 & 99.75 & 99.77 & 99.78 & 99.81 \\
\hline $\mathrm{Li}$ & 44 & 54 & 31 & 96 & 39 & 32 & 59 & 58 & 31 & 35 \\
\hline $\mathrm{Be}$ & 0.9 & 1.3 & 1.5 & 3.8 & 2.0 & 1.7 & 3.2 & 3.1 & 2.2 & 2.9 \\
\hline $\mathrm{Sc}$ & 12.3 & 9.5 & 11.7 & 21.5 & 14.3 & 7.7 & 13.9 & 19.4 & 13.7 & 8.5 \\
\hline V & 97 & 77 & 97 & 161 & 114 & 54 & 99 & 145 & 106 & 71 \\
\hline $\mathrm{Cr}$ & 76 & 71 & 73 & 190 & 96 & 48 & 64 & 91 & 57 & 73 \\
\hline Co & 15 & 9 & 11 & 26 & 13 & 7 & 13 & 19 & 11 & 9 \\
\hline $\mathrm{Ni}$ & 50 & 22 & 26 & 48 & 29 & 14 & 28 & 42 & 29 & 19 \\
\hline $\mathrm{Cu}$ & 27 & 9 & 20 & 73 & 18 & 10 & 21 & 51 & 20 & 24 \\
\hline $\mathrm{Zn}$ & 57 & 60 & 75 & 131 & 75 & 67 & 93 & 120 & 93 & 78 \\
\hline $\mathrm{Y}$ & 16 & 21 & 30 & 31 & 34 & 24 & 33 & 35 & 29 & 18 \\
\hline $\mathrm{Zr}$ & 121 & 207 & 184 & 169 & 201 & 216 & 228 & 189 & 210 & 182 \\
\hline $\mathrm{Nb}$ & 5 & 10 & 11 & 18 & 14 & 13 & 17 & 19 & 16 & 14 \\
\hline Mo & 0.3 & 0.1 & 0.3 & 0.1 & 0.3 & 0.1 & 0.2 & 2.0 & 0.3 & 0.2 \\
\hline $\mathrm{Cd}$ & 0.1 & 0.0 & 0.2 & 0.1 & 0.1 & 0.1 & 0.0 & 0.0 & 0.0 & 0.0 \\
\hline $\mathrm{Sn}$ & 0.5 & 0.1 & 0.2 & 3.7 & 2.7 & 1.1 & 3.1 & 4.1 & 1.1 & 0.0 \\
\hline $\mathrm{Sb}$ & 0.1 & 0.2 & 0.5 & 0.7 & 0.8 & 0.4 & 0.6 & 0.3 & 0.7 & 1.2 \\
\hline $\mathrm{Rb}$ & 59 & 103 & 103 & 219 & 117 & 84 & 174 & 249 & 125 & 81 \\
\hline $\mathrm{Sr}$ & 345 & 116 & 222 & 148 & 186 & 215 & 226 & 98 & 58 & 133 \\
\hline Cs & 5.8 & 5.9 & 13.0 & 14.7 & 6.2 & 4.6 & 10.2 & 16.4 & 5.5 & 7.5 \\
\hline $\mathrm{Ba}$ & 330 & 537 & 523 & 809 & 546 & 457 & 595 & 415 & 338 & 376 \\
\hline La & 21 & 32 & 34 & 43 & 42 & 37 & 50 & 44 & 38 & 23 \\
\hline $\mathrm{Ce}$ & 40 & 67 & 71 & 92 & 87 & 74 & 103 & 92 & 79 & 46 \\
\hline $\mathrm{Pr}$ & 5.2 & 8.2 & 8.7 & 10.6 & 10.9 & 8.9 & 12.4 & 10.9 & 9.6 & 5.8 \\
\hline $\mathrm{Nd}$ & 20.0 & 30.2 & 33.4 & 38.1 & 40.5 & 32.4 & 44.9 & 40.1 & 35.8 & 21.7 \\
\hline $\mathrm{Sm}$ & 3.7 & 6.0 & 6.9 & 7.4 & 8.1 & 6.1 & 8.8 & 7.7 & 6.9 & 4.4 \\
\hline Eu & 1.0 & 1.2 & 1.3 & 1.3 & 1.5 & 1.0 & 1.5 & 1.4 & 1.2 & 0.9 \\
\hline Gd & 3.1 & 5.4 & 6.6 & 6.9 & 7.7 & 5.1 & 8.0 & 6.7 & 6.0 & 3.9 \\
\hline Tb & 0.4 & 0.8 & 1.0 & 1.0 & 1.1 & 0.7 & 1.1 & 1.0 & 0.9 & 0.6 \\
\hline Dy & 2.5 & 4.2 & 5.8 & 5.9 & 6.7 & 4.3 & 6.6 & 6.0 & 5.0 & 3.7 \\
\hline $\mathrm{Ho}$ & 0.5 & 0.8 & 1.2 & 1.2 & 1.4 & 0.9 & 1.3 & 1.2 & 1.0 & 0.8 \\
\hline $\mathrm{Er}$ & 1.4 & 2.2 & 3.4 & 3.5 & 3.9 & 2.4 & 3.7 & 3.4 & 2.9 & 2.3 \\
\hline $\mathrm{Tm}$ & 0.2 & 0.3 & 0.5 & 0.5 & 0.6 & 0.3 & 0.5 & 0.5 & 0.4 & 0.3 \\
\hline $\mathrm{Yb}$ & 1.3 & 2.0 & 3.2 & 3.5 & 3.7 & 2.3 & 3.6 & 3.3 & 2.8 & 2.3 \\
\hline Lu & 0.2 & 0.3 & 0.5 & 0.5 & 0.5 & 0.3 & 0.5 & 0.5 & 0.4 & 0.3 \\
\hline $\mathrm{Hf}$ & 2.9 & 5.3 & 4.8 & 4.8 & 5.8 & 5.7 & 6.7 & 4.9 & 5.5 & 4.8 \\
\hline $\mathrm{Ta}$ & 0.3 & 0.4 & 0.3 & 1.4 & 1.1 & 0.9 & 1.4 & 1.3 & 1.1 & 0.8 \\
\hline W & 0.3 & 0.2 & 0.3 & 4.2 & 1.9 & 1.1 & 2.1 & 4.4 & 1.9 & 0.7 \\
\hline $\mathrm{Ti}$ & 0.3 & 0.5 & 0.5 & 1.1 & 0.7 & 0.4 & 0.9 & 1.0 & 0.5 & 0.6 \\
\hline $\mathrm{Pb}$ & 10.7 & 15.8 & 16.7 & 27.5 & 17.8 & 18.1 & 29.8 & 24.1 & 20.6 & 26.5 \\
\hline $\mathrm{Bi}$ & 0.1 & 0.1 & 0.2 & 0.7 & 0.2 & 0.1 & 0.3 & 1.3 & 0.4 & 0.3 \\
\hline Th & 3.1 & 11.2 & 10.3 & 15.2 & 12.4 & 11.3 & 16.7 & 16.3 & 12.4 & 8.0 \\
\hline$U$ & 1.2 & 2.4 & 2.4 & 3.9 & 2.6 & 2.6 & 3.5 & 2.4 & 2.7 & 1.8 \\
\hline Chondrit normi & Werte (Ta & und McLe & (1985) & & & & & & & \\
\hline La & 57.3 & 88.0 & 91.6 & 117.6 & 115.8 & 100.9 & 136.8 & 119.8 & 103.2 & 61.4 \\
\hline $\mathrm{Ce}$ & 42.1 & 69.9 & 73.9 & 95.8 & 90.9 & 77.8 & 107.3 & 96.2 & 82.5 & 48.1 \\
\hline $\mathrm{Pr}$ & 38.1 & 59.5 & 63.9 & 77.2 & 79.6 & 64.7 & 90.7 & 79.4 & 69.8 & 42.1 \\
\hline $\mathrm{Nd}$ & 28.1 & 42.5 & 47.0 & 53.6 & 56.9 & 45.6 & 63.2 & 56.4 & 50.3 & 30.6 \\
\hline $\mathrm{Sm}$ & 15.9 & 25.8 & 29.7 & 32.0 & 35.2 & 26.5 & 38.0 & 33.5 & 30.0 & 19.0 \\
\hline Eu & 11.2 & 13.6 & 14.7 & 14.6 & 17.2 & 11.8 & 17.2 & 15.5 & 13.4 & 9.8 \\
\hline Gd & 10.0 & 17.6 & 21.5 & 22.6 & 25.3 & 16.7 & 26.2 & 22.0 & 19.5 & 12.8 \\
\hline $\mathrm{Tb}$ & 7.6 & 13.1 & 16.7 & 17.2 & 19.7 & 12.9 & 19.5 & 17.5 & 15.2 & 10.6 \\
\hline Dy & 6.5 & 10.9 & 15.1 & 15.5 & 17.5 & 11.4 & 17.3 & 15.7 & 13.1 & 9.7 \\
\hline Ho & 5.9 & 9.5 & 13.9 & 14.4 & 15.9 & 10.0 & 15.3 & 14.1 & 11.8 & 9.0 \\
\hline $\mathrm{Er}$ & 5.6 & 8.7 & 13.6 & 14.2 & 15.7 & 9.5 & 15.0 & 13.8 & 11.6 & 9.0 \\
\hline $\mathrm{Tm}$ & 5.5 & 8.5 & 13.3 & 14.3 & 15.5 & 9.7 & 14.7 & 13.7 & 11.9 & 9.3 \\
\hline $\mathrm{Yb}$ & 5.2 & 8.1 & 13.1 & 14.0 & 15.0 & 9.4 & 14.6 & 13.4 & 11.4 & 9.4 \\
\hline Lu & 5.1 & 7.9 & 12.6 & 13.1 & 14.2 & 8.8 & 13.4 & 13.0 & 11.3 & 9.1 \\
\hline $\mathrm{Cr} N$ & 0.78 & 0.92 & 0.75 & 1.18 & 0.85 & 0.90 & 0.65 & 0.63 & 0.54 & 1.03 \\
\hline $\mathrm{Th} / \mathrm{Sc}$ & 0.25 & 1.18 & 0.89 & 0.70 & 0.87 & 1.48 & 1.21 & 0.84 & 0.90 & 0.94 \\
\hline $\mathrm{La} / \mathrm{Th}$ & 6.8 & 2.9 & 3.3 & 2.8 & 3.4 & 3.3 & 3.0 & 2.7 & 3.1 & 2.8 \\
\hline $\mathrm{Ti} / \mathrm{Zr}$ & 65.0 & 35.1 & 42.4 & 32.6 & 43.7 & 22.3 & 27.1 & 42.3 & 27.6 & 39.7 \\
\hline $\mathrm{La} / \mathrm{Sc}$ & 1.7 & 3.4 & 2.9 & 2.0 & 3.0 & 4.8 & 3.6 & 2.3 & 2.8 & 2.6 \\
\hline $\mathrm{Cr} / \mathrm{Ni}$ & 1.5 & 3.3 & 2.8 & 3.9 & 3.3 & 3.3 & 2.3 & 2.2 & 1.9 & 3.8 \\
\hline $\mathrm{Y} / \mathrm{Ni}$ & 0.31 & 0.99 & 1.17 & 0.64 & 1.18 & 1.68 & 1.18 & 0.84 & 1.00 & 0.93 \\
\hline $\mathrm{Zr} / \mathrm{Sc}$ & 9.8 & 21.8 & 15.7 & 7.9 & 14.1 & 28.3 & 16.4 & 9.8 & 15.3 & 21.4 \\
\hline $\mathrm{Nb} / \mathrm{Y}$ & 0.3 & 0.5 & 0.4 & 0.6 & 0.4 & 0.5 & 0.5 & 0.5 & 0.5 & 0.8 \\
\hline $\mathrm{Th} / \mathrm{U}$ & 2.5 & 4.7 & 4.3 & 3.9 & 4.7 & 4.4 & 4.8 & 6.9 & 4.5 & 4.5 \\
\hline $\mathrm{La}_{N} / \mathrm{Sm}_{N}$ & 3.6 & 3.4 & 3.1 & 3.7 & 3.3 & 3.8 & 3.6 & 3.6 & 3.4 & 3.2 \\
\hline $\mathrm{La}_{N} / \mathrm{Yb}_{\mathrm{N}}$ & 11.1 & 10.8 & 7.0 & 8.4 & 7.7 & 10.7 & 9.4 & 8.9 & 9.0 & 6.5 \\
\hline $\mathrm{Eu} / \mathrm{Eu}^{*}$ & 0.88 & 0.64 & 0.58 & 0.54 & 0.58 & 0.56 & 0.54 & 0.57 & 0.55 & 0.63 \\
\hline $\mathrm{Ce} / \mathrm{Ce}^{*}$ & 0.93 & 1.01 & 1.01 & 1.06 & 1.00 & 1.01 & 1.01 & 1.03 & 1.02 & 0.99 \\
\hline$\Sigma$ LREE & 90 & 144 & 153 & 191 & 189 & 159 & 219 & 195 & 169 & 100 \\
\hline ¿REE & 101 & 161 & 177 & 215 & 216 & 176 & 246 & 219 & 190 & 116 \\
\hline
\end{tabular}


Anhang 2: Sm-Nd Daten der (Meta)Sedimente der unterschiedlichen Einheiten der östlichen Sierras Pampeanas und der Puncoviscana Formation der Cordillera Oriental. Die $\mathrm{T}_{\mathrm{DM}}$ Modellalter sind nach dem Modell von Goldstein et al. (1984) mit ${ }^{143} \mathrm{Nd} /{ }^{144} \mathrm{Nd}=0.513151$ und ${ }^{147} \mathrm{Sm} /{ }^{144} \mathrm{Nd}=0.214$ berechnet worden. Der Fraktionierungsindex $\quad\left(\mathrm{f}_{\mathrm{Sm} / \mathrm{Nd}}\right)$ wurde folgendermaßen berechnet: $\mathrm{f}_{\mathrm{Sm} / \mathrm{Nd}} \quad=$ $\left[\left({ }^{147} \mathrm{Sm} /{ }^{144} \mathrm{Nd}\right)_{\text {sample }} /\left({ }^{147} \mathrm{Sm} /{ }^{144} \mathrm{Nd}\right)_{\mathrm{CHUR}}\right]-1$ mit $\left({ }^{147} \mathrm{Sm} /{ }^{144} \mathrm{Nd}\right)_{\mathrm{CHUR}}=0.1967$ nach DePaolo und Wasserburg (1976).

\begin{tabular}{|c|c|c|c|c|c|c|c|c|c|}
\hline \multicolumn{10}{|c|}{$\begin{array}{l}\text { sample Sm (ppm) Nd (ppm) } \\
\text { Nogoli Metamorphic Complex }\end{array}$} \\
\hline A7-06 & 5.932 & 30.613 & 0.117 & 0.512069 & 0.000014 & 1.70 & -11.1 & -5.6 & -0.40 \\
\hline A8-06 & 6.239 & 32.640 & 0.116 & 0.512076 & 0.000014 & 1.66 & -11.0 & -5.4 & -0.41 \\
\hline A16-06 & 6.514 & 34.466 & 0.114 & 0.512073 & 0.000011 & 1.64 & -11.0 & -5.3 & -0.42 \\
\hline \multicolumn{10}{|c|}{ Pringles Metamorphic Complex } \\
\hline A13-06 & 3.608 & 18.090 & 0.121 & 0.512024 & 0.000010 & 1.83 & -12.0 & -6.7 & -0.39 \\
\hline A14-06 & 7.318 & 38.692 & 0.114 & 0.512077 & 0.000019 & 1.64 & -11.0 & -5.3 & -0.42 \\
\hline A15-06 & 9.854 & 52.718 & 0.113 & 0.512040 & 0.000009 & 1.67 & -11.7 & -5.9 & -0.43 \\
\hline A18-06 & 5.775 & 29.678 & 0.118 & 0.512050 & 0.000108 & 1.74 & -11.5 & -6.0 & -0.40 \\
\hline \multicolumn{10}{|c|}{ Conlara Metamorphic Complex } \\
\hline A61-05 & 7.859 & 38.857 & 0.122 & 0.512223 & 0.000014 & 1.54 & -8.1 & -3.0 & -0.38 \\
\hline A73-05 & 7.364 & 37.539 & 0.118 & 0.512117 & 0.000014 & 1.65 & -10.2 & -4.8 & -0.40 \\
\hline A9-06 & 5.892 & 29.871 & 0.119 & 0.512116 & 0.000022 & 1.66 & -10.2 & -4.8 & -0.39 \\
\hline A10-06 & 9368 & .47 .522 & 0.119 & 0.512094 & 0.000013 & 1.69 & -10.6 & -5.3 & -0.39 \\
\hline \multicolumn{10}{|c|}{ San Luis Formation } \\
\hline A7-01 & 9.221 & 50.831 & 0.110 & 0.511958 & 0.000016 & 1.74 & -13.3 & -7.3 & -0.44 \\
\hline A9-01 & 5.553 & 28.922 & 0.116 & 0.511954 & 0.000008 & 1.86 & -13.4 & -7.8 & -0.41 \\
\hline A17-01 & 6.411 & 34.053 & 0.113 & 0.512007 & 0.000007 & 1.74 & -12.3 & -6.6 & -0.42 \\
\hline A30-01 & 5.780 & 29.241 & 0.119 & 0.512108 & 0.000014 & 1.68 & -10.3 & -5.0 & -0.39 \\
\hline A12-06 & 4.965 & 25.607 & 0.117 & 0.511994 & $0.0 c$ & 1.82 & -12.6 & -7.1 & -0.40 \\
\hline \multicolumn{10}{|c|}{ Puncoviscana Formation } \\
\hline A85-06 & 6.453 & 34.098 & 0.114 & 0.512084 & 0.000013 & 1.63 & -10.8 & -5.1 & -0.42 \\
\hline A86-06 & 7.621 & 36.553 & 0.126 & 0.512190 & 0.000032 & 1.66 & -8.7 & -3.9 & -0.36 \\
\hline A87-06 & 6.155 & 30.114 & 0.124 & 0.512103 & 0.000118 & 1.76 & -10.4 & -5.4 & -0.37 \\
\hline A88-06 & 5.475 & 27.822 & 0.119 & 0.512050 & 0.000011 & 1.76 & -11.5 & -6.1 & -0.40 \\
\hline A90-06 & 6.284 & 31.868 & 0.119 & 0.512044 & 0.000012 & 1.78 & -11.6 & -6.2 & -0.39 \\
\hline A91-06 & 5.391 & 27.274 & 0.120 & 0.512071 & 0.000016 & 1.74 & -11.1 & -5.7 & -0.39 \\
\hline A93-06 & 5.399 & 28.543 & 0.114 & 0.512022 & 0.000016 & 1.72 & -12.0 & -6.3 & -0.42 \\
\hline A94-06 & 5.604 & 28.170 & 0.120 & 0.512043 & 0.000021 & 1.80 & -11.6 & -6.3 & -0.39 \\
\hline A97-06 & 7.152 & 36.734 & 0.118 & 0.512065 & 0.000016 & 1.72 & -11.2 & -5.7 & -0.40 \\
\hline \multicolumn{10}{|c|}{ Sierras de Chepes } \\
\hline A31-06 & 7.703 & 3 & 53 & 003 & 0.0 & 1.77 & -12.3 & -6.8 & -0.41 \\
\hline A35-06 & 6.287 & 32.615 & 0.1165 & 0.511986 & 0.000016 & 1.82 & -12.3 & -7.2 & -0.41 \\
\hline A37-06 & 14.541 & 38.301 & 0.1187 & 0.511968 & 0.000016 & 1.89 & -13.0 & -7.7 & -0.40 \\
\hline A41-06 & 5.154 & 25.820 & 0.1207 & 0.512032 & 0.000013 & 1.82 & -11.8 & -6.6 & -0.39 \\
\hline \multicolumn{10}{|c|}{ Sierras de Córdoba } \\
\hline A60-06 & 8.470 & 44.785 & 0.1143 & 0.512069 & 0.000013 & 1.65 & -11.1 & -5.4 & -0.42 \\
\hline A61-06 & 7.712 & 35.046 & 0.1330 & 0.512148 & 0.000014 & 1.88 & -9.5 & -5.2 & -0.32 \\
\hline A62-06 & 5.896 & 29.784 & 0.1197 & 0.512157 & 0.000018 & 1.60 & -9.3 & -4.1 & -0.39 \\
\hline A66-06 & 6.855 & 34.488 & 0.1202 & 0.512072 & 0.000022 & 1.75 & -11.0 & -5.8 & -0.39 \\
\hline A68-06 & 11.122 & 54.845 & 0.1226 & 0.512210 & 0.000009 & 1.57 & -8.3 & -3.2 & -0.38 \\
\hline A70-06 & 12.569 & 61.019 & 0.1245 & 0.512196 & 0.000049 & 1.62 & -8.6 & -3.6 & -0.37 \\
\hline A74-06 & 5.924 & 29.343 & 0.1221 & 0.512183 & 0.000026 & 1.60 & -8.8 & -3.7 & -0.38 \\
\hline A76-06 & 8.400 & 42.838 & 0.1185 & 0.512097 & 0.000014 & 1.68 & -10.5 & -5.2 & -0.40 \\
\hline A77-06 & 6.686 & 32.789 & 0.1233 & 0.512177 & 0.000054 & 1.63 & -8.9 & -3.9 & -0.37 \\
\hline A78-06 & 9.366 & 48.744 & 0.1162 & 0.512068 & 0.000011 & 1.68 & -11.1 & -5.6 & -0.41 \\
\hline A79-06 & 9.612 & 47.774 & 0.1216 & 0.512181 & 0.000014 & 1.60 & -8.9 & -3.7 & -0.38 \\
\hline A80-06 & 8.674 & 41.249 & 0.1271 & 0.512075 & 0.000027 & 1.88 & -10.9 & -6.2 & -0.35 \\
\hline \multicolumn{10}{|c|}{ Sierra Norte } \\
\hline A81-06 & 6.084 & 31.797 & 0.1157 & 0.511972 & 0.000015 & 1.82 & -12.9 & -7.4 & -0.41 \\
\hline A100-06 & 5.819 & 30.362 & 0.1159 & 0.511986 & 0.000013 & 1.81 & -12.7 & -7.2 & -0.41 \\
\hline A101-06 & 7.034 & 34.954 & 0.1217 & 0.512066 & 0.000012 & 1.79 & -11.1 & -6.0 & -0.38 \\
\hline A102-06 & 6.779 & 35.476 & 0.1155 & 0.512072 & 0.000022 & 1.67 & -11.0 & -5.4 & -0.41 \\
\hline A104-06 & 5.793 & 31.258 & 0.1121 & 0.511999 & 0.000019 & 1.72 & -12.4 & -6.6 & -0.43 \\
\hline A105-06 & 6.575 & 34.126 & 0.1165 & 0.511967 & 0.000016 & 1.85 & -13.0 & -7.6 & -0.41 \\
\hline A107-06 & 6.880 & 35.488 & 0.1172 & 0.512032 & 0.000012 & 1.76 & -11.8 & -6.3 & -0.40 \\
\hline
\end{tabular}


Anhang 3: Gesamtgesteins Pb-Isotopie aller untersuchter Einheiten der östlichen Sierras Pampeanas und der Cordillera Oriental.

\begin{tabular}{|c|c|c|c|c|c|c|c|c|c|c|}
\hline sample & ${ }^{206} \mathrm{~Pb} /{ }^{204} \mathrm{~Pb}$ & $\pm 2 \mathrm{~s}$ & ${ }^{207} \mathrm{~Pb} /{ }^{204} \mathrm{~Pb}$ & $\pm 2 \mathrm{~s}$ & ${ }^{208} \mathrm{~Pb} /{ }^{204} \mathrm{~Pb}$ & $\pm 2 \mathrm{~s}$ & ${ }^{207} \mathrm{~Pb} /{ }^{206} \mathrm{~Pb}$ & $\pm 2 \mathrm{~s}$ & ${ }^{208} \mathrm{~Pb} /{ }^{206} \mathrm{~Pb}$ & $\pm 2 \mathrm{~s}$ \\
\hline \multicolumn{11}{|c|}{ Conlara Metamorphic Complex } \\
\hline A61-05 & 19.310 & 0.012 & 15.716 & 0.011 & 39.368 & 0.033 & 0.8139 & 0.0002 & 2.0387 & 0.0007 \\
\hline A9-06 & 18.785 & 0.009 & 15.676 & 0.010 & 38.671 & 0.029 & 0.8345 & 0.0002 & 2.0586 & 0.0007 \\
\hline A10-06 & 21.061 & 0.014 & 15.835 & 0.012 & 42.013 & 0.039 & 0.7519 & 0.0002 & 1.9948 & 0.0010 \\
\hline \multicolumn{11}{|c|}{ Pringles Metamorphic Complex } \\
\hline A13-06 & 19.352 & 0.012 & 15.743 & 0.012 & 39.878 & 0.034 & 0.8135 & 0.0002 & 2.0607 & 0.0007 \\
\hline A14-06 & 18.816 & 0.014 & 15.683 & 0.013 & 39.185 & 0.039 & 0.8335 & 0.0002 & 2.0825 & 0.0011 \\
\hline A15-06 & 18.642 & 0.012 & 15.659 & 0.012 & 39.314 & 0.035 & 0.8400 & 0.0002 & 2.1089 & $0.000 s$ \\
\hline A18-06 & 19.259 & 0.009 & 15.712 & 0.010 & 39.613 & 0.031 & 0.8158 & 0.0002 & 2.0568 & 0.0008 \\
\hline \multicolumn{11}{|c|}{ Nogolí Metamorphic Complex } \\
\hline A7-06 & 19.254 & 0.012 & 15.706 & 0.011 & 39.402 & 0.033 & 0.8157 & 0.0002 & 2.0465 & 0.0007 \\
\hline A8-06 & 18.941 & 0.022 & 15.698 & 0.020 & 39.343 & 0.053 & 0.8288 & 0.0003 & 2.0771 & 0.0010 \\
\hline A16-06 & 18.673 & 0.008 & 15.679 & 0.009 & 39.009 & 0.029 & 0.8396 & 0.0002 & 2.0890 & 0.0008 \\
\hline \multicolumn{11}{|c|}{ San Luis Formation } \\
\hline A11-01 & 19.840 & 0.013 & 15.731 & 0.012 & 40.171 & 0.035 & 0.7929 & 0.0002 & 2.0248 & 0.0007 \\
\hline A32-01 & 18.726 & 0.010 & 15.669 & 0.010 & 38.968 & 0.031 & 0.8367 & 0.0002 & 2.0810 & 0.0008 \\
\hline A34-01 & 19.262 & 0.009 & 15.696 & 0.010 & 39.840 & 0.031 & 0.8149 & 0.0002 & 2.0684 & 0.0008 \\
\hline A12-06 & 18.880 & 0.010 & 15.744 & 0.011 & 39.177 & 0.032 & 0.8339 & 0.0002 & 2.0750 & 0.0008 \\
\hline \multicolumn{11}{|c|}{ Puncoviscana Formation } \\
\hline A86-06 & 19.012 & 0.009 & 15.679 & 0.009 & 38.822 & 0.029 & 0.8247 & 0.0002 & 2.0420 & 0.0007 \\
\hline A87-06 & 19.158 & 0.012 & 15.695 & 0.011 & 39.358 & 0.033 & 0.8193 & 0.0002 & 2.0544 & 0.0007 \\
\hline A88-06 & 18.931 & 0.014 & 15.763 & 0.013 & 39.261 & 0.037 & 0.8327 & 0.0002 & 2.0739 & 0.0007 \\
\hline A89-06 & 18.866 & 0.010 & 15.680 & 0.010 & 39.086 & 0.031 & 0.8311 & 0.0002 & 2.0718 & 0.0007 \\
\hline A91-06 & 18.903 & 0.008 & 15.679 & 0.009 & 39.078 & 0.028 & 0.8295 & 0.0002 & 2.0674 & 0.0007 \\
\hline A93-06 & 18.352 & 0.009 & 15.631 & 0.010 & 38.588 & 0.029 & 0.8517 & 0.0002 & 2.1027 & 0.0007 \\
\hline A94-06 & 18.700 & 0.018 & 15.678 & 0.016 & 38.741 & 0.044 & 0.8384 & 0.0002 & 2.0718 & $0.000 s$ \\
\hline A96-06 & 19.520 & 0.013 & 15.725 & 0.012 & 39.852 & 0.036 & 0.8056 & 0.0002 & 2.0416 & 0.0008 \\
\hline A97-06 & 18.851 & 0.010 & 15.673 & 0.010 & 38.873 & 0.030 & 0.8314 & 0.0002 & 2.0621 & 0.0008 \\
\hline \multicolumn{11}{|c|}{ Sierras de Chepes } \\
\hline A31-06 & 19.150 & 0.013 & 15.703 & 0.012 & 39.462 & 0.034 & 0.8200 & 0.0002 & 2.0606 & 0.0007 \\
\hline A35-06 & 18.840 & 0.009 & 15.674 & 0.010 & 39.101 & 0.030 & 0.8320 & 0.0002 & 2.0754 & 0.0008 \\
\hline A37-06 & 19.862 & 0.015 & 15.726 & 0.014 & 40.559 & 0.041 & 0.7918 & 0.0002 & 2.0421 & $0.000 s$ \\
\hline A41-06 & 18.763 & 0.011 & 15.671 & 0.011 & 38.914 & 0.032 & 0.8352 & 0.0002 & 2.0740 & 0.0007 \\
\hline \multicolumn{11}{|c|}{ Sierras de Córdoba } \\
\hline A60-06 & 18.606 & 0.010 & 15.658 & 0.011 & 39.534 & 0.032 & 0.8416 & 0.0002 & 2.1249 & 0.0008 \\
\hline A62-06 & 18.904 & 0.008 & 15.687 & 0.009 & 38.829 & 0.028 & 0.8299 & 0.0001 & 2.0541 & 0.0007 \\
\hline A66-06 & 19.194 & 0.016 & 15.698 & 0.014 & 39.263 & 0.040 & 0.8179 & 0.0002 & 2.0456 & 0.0007 \\
\hline A68-06 & 20.658 & 0.010 & 15.784 & 0.010 & 40.690 & 0.032 & 0.7641 & 0.0002 & 1.9698 & 0.0008 \\
\hline A70-06 & 19.232 & 0.011 & 15.710 & 0.011 & 39.402 & 0.033 & 0.8169 & 0.0002 & 2.0488 & 0.0008 \\
\hline A73-06 & 19.394 & 0.021 & 15.696 & 0.018 & 39.324 & 0.049 & 0.8093 & 0.0002 & 2.0277 & 0.0008 \\
\hline A75-06 & 18.991 & 0.021 & 15.667 & 0.018 & 38.723 & 0.049 & 0.8250 & 0.0002 & 2.0390 & $0.000 s$ \\
\hline A76-06 & 18.847 & 0.011 & 15.675 & 0.011 & 39.424 & 0.033 & 0.8317 & 0.0002 & 2.0919 & 0.0008 \\
\hline A77-06 & 18.745 & 0.009 & 15.664 & 0.009 & 38.692 & 0.028 & 0.8356 & 0.0002 & 2.0642 & 0.0007 \\
\hline A78-06 & 18.796 & 0.008 & 15.680 & 0.009 & 39.496 & 0.028 & 0.8342 & 0.0001 & 2.1013 & 0.0007 \\
\hline A79-06 & 20.497 & 0.015 & 15.759 & 0.013 & 40.659 & 0.038 & 0.7688 & 0.0002 & 1.9837 & 0.0007 \\
\hline A80-06 & 18.809 & 0.010 & 15.676 & 0.010 & 39.525 & 0.030 & 0.8335 & 0.0002 & 2.1014 & 0.0007 \\
\hline \multicolumn{11}{|c|}{ Sierra Norte } \\
\hline A81-06 & 18.662 & 0.013 & 15.700 & 0.013 & 39.415 & 0.037 & 0.8413 & 0.0002 & 2.1121 & 0.0008 \\
\hline A102-06 & 18.823 & 0.012 & 15.677 & 0.012 & 38.801 & 0.034 & 0.8329 & 0.0002 & 2.0614 & 0.0008 \\
\hline A104-06 & 18.607 & 0.009 & 15.648 & 0.010 & 38.885 & 0.030 & 0.8410 & 0.0002 & 2.0898 & 0.0007 \\
\hline A105-06 & 18.532 & 0.011 & 15.655 & 0.011 & 38.813 & 0.032 & 0.8448 & 0.0002 & 2.0944 & 0.0008 \\
\hline A107-06 & 18.655 & 0.010 & 15.661 & 0.010 & 38.902 & 0.030 & 0.8395 & 0.0002 & 2.0853 & 0.0007 \\
\hline
\end{tabular}


Anhang 4.1: U-Pb SHRIMP Daten von den Zirkonen der Proben A7-06 und A8-06 aus dem Nogolí Metamorphic Complex und A73-05 aus dem Conlara Metamorphic Complex..

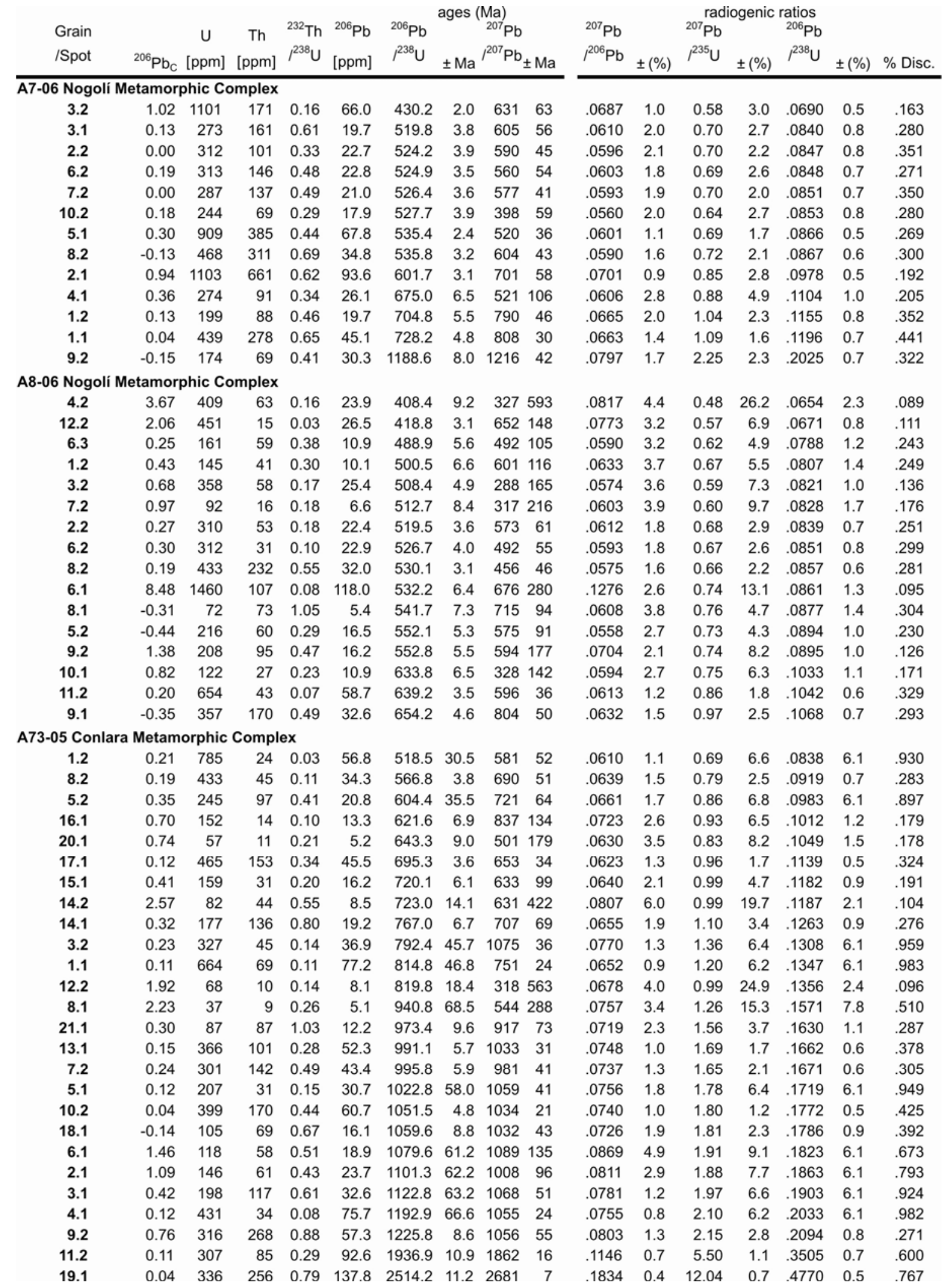


Anhang 4.2: U-Pb SHRIMP Daten von den Zirkonen der Proben A30-01 und A93-05 aus der San Luis Formation.

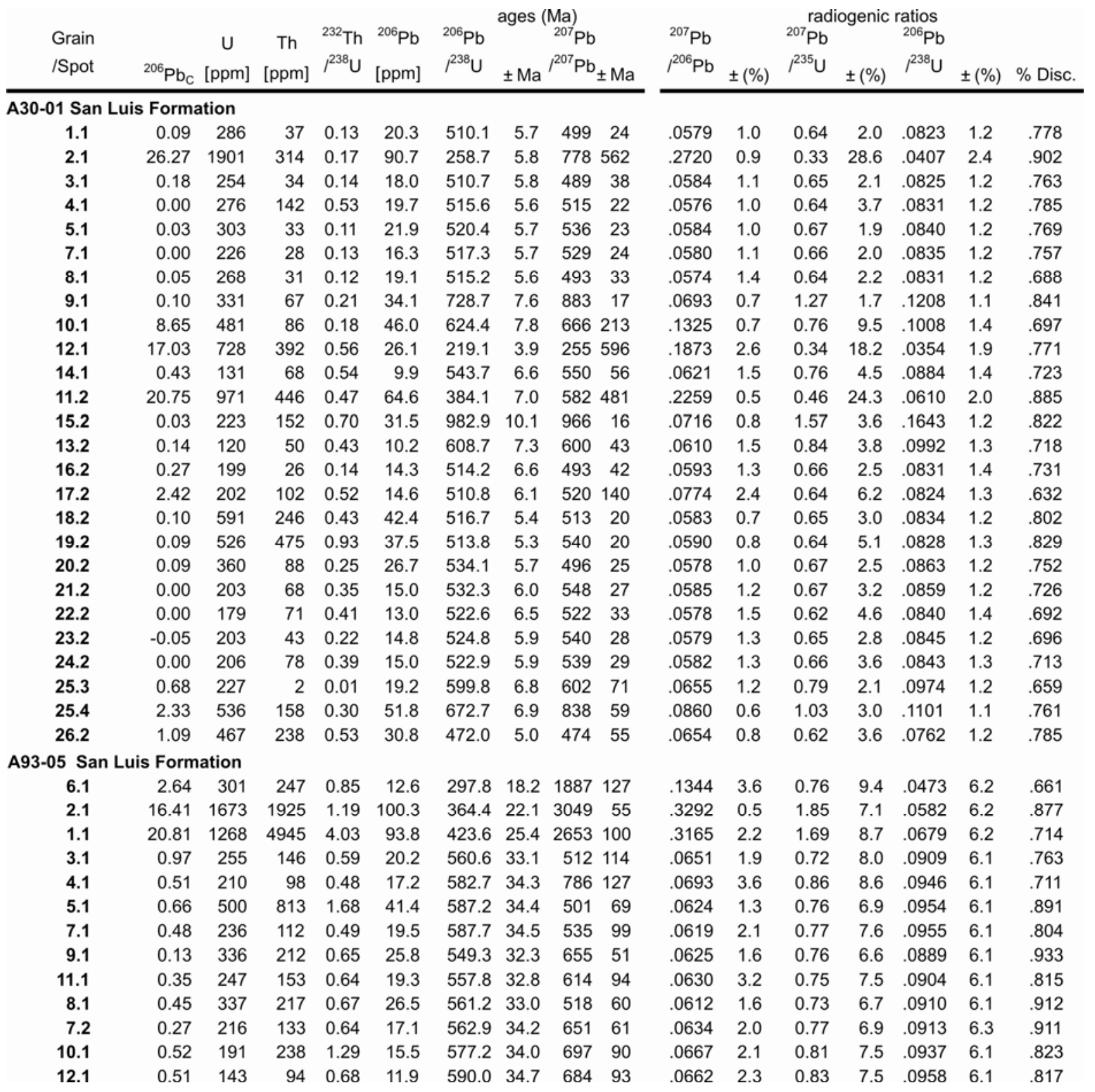


Anhang 4.3: U-Pb SHRIMP Daten von den Zirkonen den Proben A1-08 und A4-08 aus den Sierras de Chepes.

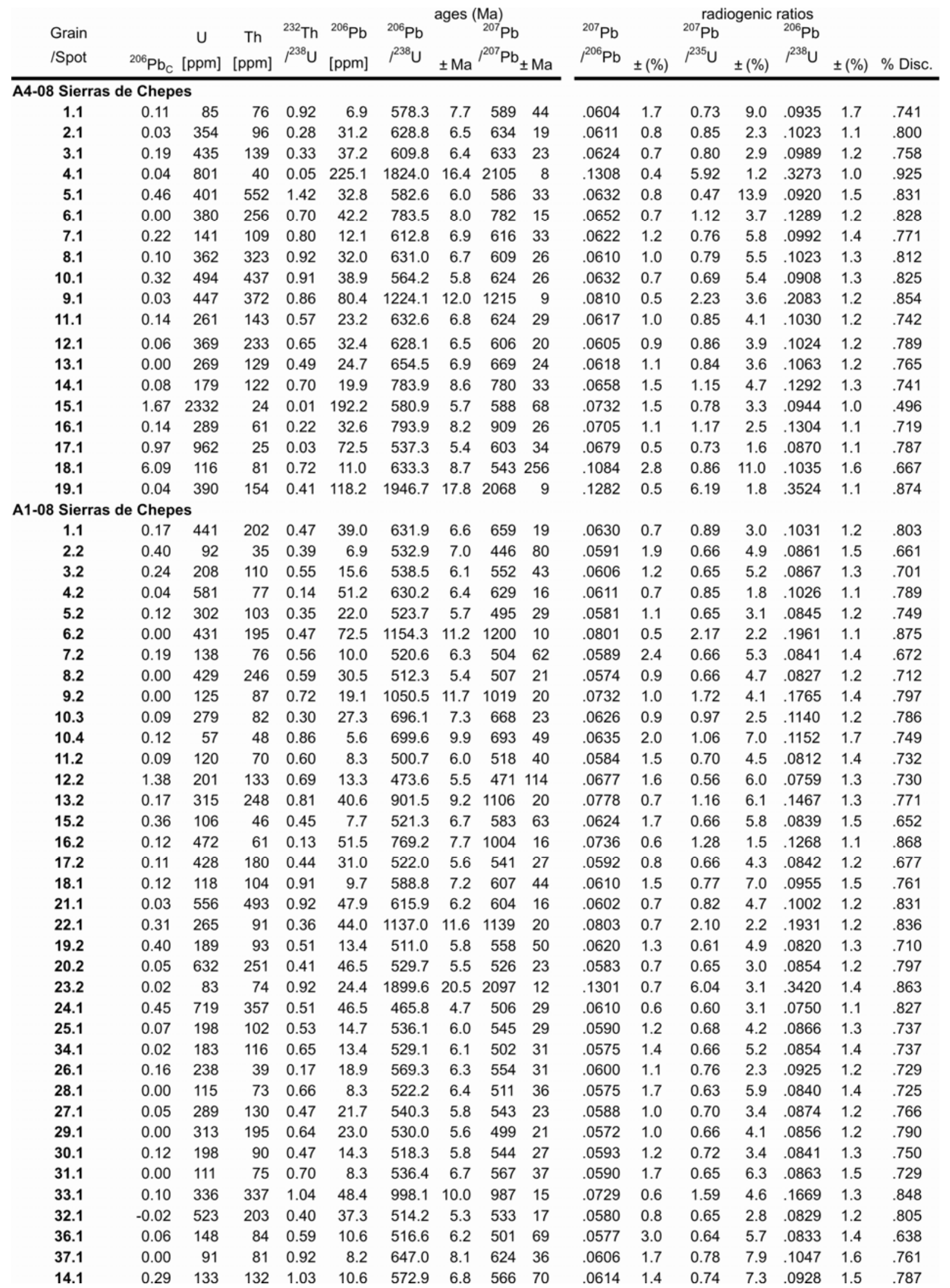


Anhang 4.4: U-Pb SHRIMP Daten von den Zirkonen der Probe A3-08 aus den Sierras de Córdoba

\begin{tabular}{|c|c|c|c|c|c|c|c|c|c|c|c|c|c|c|c|c|}
\hline $\begin{array}{l}\text { Grain } \\
\text { /Spot }\end{array}$ & \multicolumn{2}{|c|}{$\begin{array}{c}\mathrm{U} \\
{ }^{206} \mathrm{~Pb}_{\mathrm{C}}[\mathrm{ppm}] \\
\end{array}$} & $\begin{array}{c}\text { Th } \\
{[\mathrm{ppm}]}\end{array}$ & $\begin{array}{l}{ }^{232} \mathrm{Th} \\
{ }^{238} \mathrm{U}\end{array}$ & $\begin{array}{l}{ }^{206} \mathrm{~Pb} \\
{[\mathrm{ppm}]}\end{array}$ & $\begin{array}{l}{ }^{206} \mathrm{~Pb} \\
\rho^{238} \mathrm{U}\end{array}$ & $\begin{array}{l}\text { ages } \\
\pm \mathrm{Ma}\end{array}$ & $\begin{array}{l}(\mathrm{Ma}) \\
{ }^{207} \mathrm{~Pb} \\
\rho^{207} \mathrm{~Pb}\end{array}$ & & $\begin{array}{l}{ }^{207} \mathrm{~Pb} \\
{ }^{206} \mathrm{~Pb}\end{array}$ & $\pm(\%$ & $\begin{array}{l}\text { radi } \\
{ }^{207} \mathrm{~Pb} \\
\rho^{235} \mathrm{U}\end{array}$ & ogenic & $\begin{array}{l}\text { ratios } \\
{ }^{206} \mathrm{~Pb} \\
{ }^{238} \mathrm{U}\end{array}$ & $\pm(\%)$ & $\% \mathrm{D}$ \\
\hline \multicolumn{17}{|c|}{ A3-08 Sierras de Córdoba } \\
\hline 34.1 & 0.53 & 91 & 40 & 0.45 & 10.5 & 807.4 & 11.8 & 844 & 89 & .0716 & 1.8 & 1.22 & 4.9 & .1333 & 1.7 & .707 \\
\hline 33.1 & 0.71 & 79 & 19 & 0.26 & 11.1 & 975.6 & 14.2 & 980 & 75 & .0778 & 1.7 & 1.64 & 3.7 & .1636 & 1.6 & .700 \\
\hline 31.1 & 0.33 & 961 & 9 & 0.01 & 77.4 & 575.8 & 5.9 & 588 & 28 & .0622 & 0.7 & 0.77 & 1.4 & .0934 & 1.1 & .810 \\
\hline 32.1 & 0.16 & 654 & 462 & 0.73 & 67.2 & 726.8 & 7.5 & 756 & 21 & .0657 & 0.7 & 1.03 & 3.9 & .1191 & 1.2 & .824 \\
\hline 30.1 & 9.04 & 1535 & 191 & 0.13 & 99.4 & 427.4 & 5.1 & 619 & 195 & .1331 & 0.4 & 0.57 & 7.9 & .0685 & 1.3 & .699 \\
\hline 29.1 & 0.79 & 106 & 203 & 1.97 & 14.7 & 954.3 & 13.0 & 954 & 86 & .0775 & 1.4 & 1.41 & 14.8 & .1584 & 2.1 & .865 \\
\hline 28.1 & 0.23 & 640 & 286 & 0.46 & 94.4 & 1019.2 & 10.4 & 1046 & 15 & .0761 & 0. & 1.74 & 2.7 & .1712 & 1.2 & .820 \\
\hline 27.1 & 0.12 & 163 & 30 & 0.19 & 14.5 & 635.9 & 8.0 & 611 & 45 & .0612 & 1. & 0.85 & 3.1 & .1036 & 1.4 & .667 \\
\hline 26.1 & 0.29 & 129 & 55 & 0.44 & 17.0 & 922.8 & 11.7 & 1002 & 30 & .0750 & 1. & 1.58 & 3.7 & .1542 & 1.5 & .735 \\
\hline 25.1 & 0.14 & 967 & 205 & 0.22 & 90.7 & 667.3 & 6.7 & 680 & 19 & .0633 & 0. & 0.94 & 1.9 & .1091 & 1.1 & .848 \\
\hline 24.1 & 0.00 & 139 & 114 & 0.85 & 22.6 & 1118.6 & 13.5 & 1128 & 23 & .0773 & 1. & 2.04 & 4.7 & .1896 & 1. & .802 \\
\hline 22.1 & 0.22 & 184 & 122 & 0.69 & 46.9 & 1676.6 & 18.1 & 1831 & 15 & .1138 & 0. & 4.55 & 3.6 & .2968 & 1.4 & .772 \\
\hline 21.1 & 0.00 & 54 & 18 & 0.35 & 8.6 & 1085.7 & 18.6 & 1095 & 38 & .0760 & 1. & 1.79 & 4.8 & .1824 & 20 & .725 \\
\hline 20.1 & 0.13 & 487 & 11 & 0.02 & 33.3 & 492.4 & 5.4 & 698 & 24 & .0638 & 1. & 0.63 & 1.9 & .0789 & & .697 \\
\hline 19.2 & 0.37 & 58 & 27 & 0.48 & 8.6 & 1025.5 & 15.8 & 1117 & 43 & .0799 & 1. & 1.90 & 4.6 & .1730 & 1 & .732 \\
\hline 19.1 & 0.07 & 553 & 2 & 0.00 & 37.1 & 484.7 & 5.2 & 465 & 26 & .0569 & 1. & 0.61 & 1.6 & .0781 & & .727 \\
\hline 18.1 & 0.16 & 147 & 85 & 0.60 & 45.0 & 1959.8 & 22.6 & 1938 & 14 & .1201 & 0. & 5.89 & 2.6 & .3558 & 1.4 & .880 \\
\hline 18.2 & 0.09 & 599 & 2 & 0.00 & 39.1 & 471.1 & 5.2 & 461 & 25 & .0570 & 1. & 0.59 & 1.6 & .0758 & 1.2 & .744 \\
\hline 16.1 & 0.14 & 604 & 8 & 0.01 & 39.6 & 473.2 & 5.1 & 479 & 30 & .0579 & 1. & 0.60 & 8 & .0762 & 1 & .663 \\
\hline 17.1 & 0.05 & 612 & 7 & 0.01 & 42.0 & 495.5 & 5.3 & 483 & 25 & .0572 & 1. & 0.62 & 1.6 & .0798 & 1 & .738 \\
\hline 14.1 & 0.37 & 63 & 30 & 0.49 & 6.2 & 691.6 & 11.1 & 736 & 88 & .0669 & 2. & 0.99 & 6.2 & .1132 & 1.8 & .683 \\
\hline 14.2 & 0.24 & 275 & 21 & 0.08 & 20.8 & 542.1 & 6.3 & 568 & 43 & .0610 & 1.4 & 0.70 & 2.4 & .0876 & 1.2 & .641 \\
\hline 13.1 & 0.08 & 571 & 2 & 0.00 & 36.5 & 463.0 & 5.0 & 498 & 23 & .0578 & 1.0 & 0.58 & 1.6 & .0744 & 1.1 & .736 \\
\hline 12.1 & 0.23 & 477 & 9 & 0.02 & 32.6 & 493.3 & 5.4 & 507 & 42 & .0592 & 1. & 0.62 & 1.8 & .0795 & 1.1 & .710 \\
\hline 12.2 & 0.18 & 100 & 50 & 0.51 & 15.2 & 1043.5 & 13.5 & 1074 & 32 & .0767 & 1.4 & 1.79 & 4.1 & .1755 & 1.5 & .754 \\
\hline 11.1 & 0.30 & 504 & 11 & 0.02 & 36.6 & 522.2 & 5.8 & 516 & 37 & .0600 & 1.1 & 0.66 & 1.9 & .0843 & 1.2 & .703 \\
\hline 10.1 & -0.01 & 907 & 53 & 0.06 & 93.1 & 727.3 & 7.3 & 747 & 13 & .0641 & 0.6 & 1.04 & 1.4 & .1193 & 1.1 & .865 \\
\hline 9.1 & 0.17 & 560 & 6 & 0.01 & 37.1 & 478.2 & 5.1 & 488 & 30 & .0583 & 1.0 & 0.60 & 1.6 & .0770 & 1.1 & .741 \\
\hline 9.2 & -0.52 & 26 & 2 & 0.09 & 4.2 & 1100.3 & 21.3 & 1441 & 44 & .0864 & 2.4 & 2.03 & 4.2 & .1838 & 2.2 & .652 \\
\hline 8.1 & 0.14 & 321 & 92 & 0.30 & 26.3 & 586.5 & 6.5 & 575 & 32 & .0603 & 1.1 & 0.78 & 2.8 & .0953 & 1.2 & .739 \\
\hline 7.1 & 0.03 & 336 & 133 & 0.41 & 44.0 & 916.2 & 9.7 & 988 & 18 & .0723 & 0.8 & 1.38 & 2.9 & .1516 & 1.2 & .795 \\
\hline 5.1 & 0.39 & 605 & 35 & 0.06 & 47.4 & 560.1 & 6.0 & 566 & 37 & .0622 & 0.8 & 0.74 & 1.7 & .0908 & 1.1 & .780 \\
\hline 5.2 & 2.08 & 202 & 83 & 0.43 & 18.7 & 647.5 & 7.9 & 670 & 112 & .0789 & 1.5 & 0.86 & 7.0 & .1053 & 1.4 & .643 \\
\hline 6.1 & 2.01 & 391 & 15 & 0.04 & 27.7 & 500.3 & 5.7 & 434 & 122 & .0719 & 1.0 & 0.64 & 2.6 & .0809 & 1.2 & .648 \\
\hline 6.2 & 0.16 & 112 & 35 & 0.32 & 12.9 & 809.5 & 10.7 & 778 & 40 & .0664 & 1.6 & 1.13 & 4.0 & .1332 & 1.5 & .690 \\
\hline 4.1 & 0.56 & 565 & 7 & 0.01 & 37.5 & 477.0 & 5.1 & 469 & 45 & .0610 & 0.9 & 0.60 & 2.0 & .0768 & 1.1 & .667 \\
\hline 2.2 & 0.74 & 397 & 108 & 0.28 & 35.1 & 626.8 & 7.0 & 659 & 49 & .0676 & 0.9 & 0.88 & 7.5 & .1023 & 1.3 & .609 \\
\hline 3.1 & 1.90 & 716 & 94 & 0.14 & 51.9 & 513.2 & 5.4 & 559 & 69 & .0742 & 0.7 & 0.66 & 3.1 & .0827 & 1.1 & .671 \\
\hline 1.1 & 0.07 & 162 & 95 & 0.61 & 24.6 & 1051.1 & 11.2 & 1024 & 20 & .0739 & 0.9 & 1.80 & 3.2 & .1772 & 1.3 & .815 \\
\hline 2.1 & 0.02 & 412 & 2 & 0.00 & 26.5 & 464.8 & 5.0 & 477 & 25 & .0568 & 0.9 & 0.58 & 1.5 & .0747 & 1.1 & .766 \\
\hline
\end{tabular}


- Geburt: $\quad$ 03.07.1978 in Hannover, Staatsangehörigkeit: deutsch

- Grundschule: 1985 - 1989 an der Grundschule II in Sarstedt

- Gymnasium: 1989 - 1998 am Gymnasium Andreanum in Hildesheim mit Erlangung der allgemeinen Hochschulreife

- Bundeswehr: $\quad$ September 1998 - August 1999 in Neustadt/Luttmersen

- Universität: $\quad$ Beginn des Studiums der Geowissenschaften zum Wintersemester 1999 an der Georg-August-Universität Göttingen.

Bachelorarbeit: „K-Ar Altersdatierungen an Gesteinen des KduboKenieba Inliers im Südosten Senegals.“ Bachelorabschluss im November 2002.

Diplomarbeit: „Auswirkungen von Grundwasserschwankungen auf das supraleitende Gravimeter des GGeodynamischen Observatoriums in Moxa.“ Abschluss im Juli 2005.

Der Beginn der Doktorarbeit lag im Januar 2006. 